\title{
Global, regional, and national incidence, prevalence, and years lived with disability for 301 acute and chronic diseases and injuries in 188 countries, 1990-2013: a systematic analysis for the Global Burden of Disease Study 2013
}

\author{
Global Burden of Disease Study 2013 Collaborators
}

\section{Summary}

\begin{abstract}
Background-Up-to-date evidence about levels and trends in disease and injury incidence, prevalence, and years lived with disability (YLDs) is an essential input into global, regional, and national health policies. In the Global Burden of Disease Study 2013 (GBD 2013), we estimated these quantities for acute and chronic diseases and injuries for 188 countries between 1990 and 2013.
\end{abstract}

\begin{abstract}
Methods-Estimates were calculated for disease and injury incidence, prevalence, and YLDs using GBD 2010 methods with some important refinements. Results for incidence of acute disorders and prevalence of chronic disorders are new additions to the analysis. Key improvements include expansion to the cause and sequelae list, updated systematic reviews, use of detailed injury codes, improvements to the Bayesian meta-regression method (DisMod-MR), and use of severity splits for various causes. An index of data representativeness, showing data availability, was calculated for each cause and impairment during three periods globally and at the country level for 2013. In total, 35620 distinct sources of data were used and documented to calculated estimates for 301 diseases and injuries and 2337 sequelae. The comorbidity simulation
\end{abstract}

Correspondence to: Prof Theo Vos, Institute for Health Metrics and Evaluation, 2301 5th Avenue, Suite 600, Seattle, WA 98121,

USA, tvos@uw.edu.

Collaborators listed at the end of the Article

Contributors

TV, ADL, MN, and CJLM prepared the first draft. TV, CJLM, MFM, GH, and CS finalized the draft based on comments from other authors and reviewer feedback. TV, ADL, MN, and CJLM conceived of the study and provided overall guidance. TF, RB, SB, DD,

LS, and JW performed final statistical analyses. All other authors provided data, developed models, reviewed results, provided guidance on methodology, and reviewed the manuscript.

See Online/Comment http://dx.doi.org/10.1016/S0140-6736(14)62254-6

See Online for appendix

For the Global Health Data Exchange see http://ghdx.healthdata.org

For more on DisMod-MR 2.0 see http://ihmeuw.org/dismod-ode

For the Cancer Incidence in Five Continents see http://ci5.iarc.fr/Default.aspx

For GBD Data Visualizations see http://www.healthdata.org/gbd/data-visualizations

Declaration of interests

FP-R has consulted for Astra-Zeneca, Menarini, and Pfizer, honoraria for developing educational materials from Astra-Zeneca, and Menarini, been a speaker for Astra-Zeneca, Menarini, Sociedad Espanola de Reumatologia, and has received investigational grants from Fundacion Espanola de Reumatologia, Ministerio de Sanidad (Gobierno de Espana), Asociacion de Reumatologos del Hospital de Cruces. JC reports grants from National Kidney Foundation, is a board member of the Kidney Disease Improving Global Outcomes, and has a patent on glomerular filtration rate estimation using a panel of biomarkers pending. The other authors declare no competing interests. 
provides estimates for the number of sequelae, concurrently, by individuals by country, year, age, and sex. Disability weights were updated with the addition of new population-based survey data from four countries.

Findings-Disease and injury were highly prevalent; only a small fraction of individuals had no sequelae. Comorbidity rose substantially with age and in absolute terms from 1990 to 2013. Incidence of acute sequelae were predominantly infectious diseases and short-term injuries, with over 2 billion cases of upper respiratory infections and diarrhoeal disease episodes in 2013, with the notable exception of tooth pain due to permanent caries with more than 200 million incident cases in 2013. Conversely, leading chronic sequelae were largely attributable to noncommunicable diseases, with prevalence estimates for asymptomatic permanent caries and tension-type headache of 2.4 billion and 1.6 billion, respectively. The distribution of the number of sequelae in populations varied widely across regions, with an expected relation between age and disease prevalence. YLDs for both sexes increased from 537.6 million in 1990 to 764.8 million in 2013 due to population growth and ageing, whereas the age-standardised rate decreased little from 114.87 per 1000 people to 110.31 per 1000 people between 1990 and 2013. Leading causes of YLDs included low back pain and major depressive disorder among the top ten causes of YLDs in every country. YLD rates per person, by major cause groups, indicated the main drivers of increases were due to musculoskeletal, mental, and substance use disorders, neurological disorders, and chronic respiratory diseases; however HIV/AIDS was a notable driver of increasing YLDs in sub-Saharan Africa. Also, the proportion of disability-adjusted life years due to YLDs increased globally from 21.1\% in 1990 to $31.2 \%$ in 2013.

Interpretation-Ageing of the world's population is leading to a substantial increase in the numbers of individuals with sequelae of diseases and injuries. Rates of YLDs are declining much more slowly than mortality rates. The non-fatal dimensions of disease and injury will require more and more attention from health systems. The transition to non-fatal outcomes as the dominant source of burden of disease is occurring rapidly outside of sub-Saharan Africa. Our results can guide future health initiatives through examination of epidemiological trends and a better understanding of variation across countries.

\section{Introduction}

The Global Burden of Disease Study 2013 (GBD 2013) is the first of a series of yearly updates for the GBD studies that began with estimates for 1990 and were most recently updated to 2010. The 2010 update (GBD 2010) systematically quantified prevalence of 1160 sequelae of 289 diseases and injuries across 21 regions. ${ }^{1}$ National estimates for 187 countries were also derived on the basis of global and regional statistical analyses. ${ }^{1}$ The metrics of years lived with disability (YLDs), equal to the sum of prevalence multiplied by the general public's assessment of the severity of health loss, was used to explore patterns over time, age, sex, and geography. ${ }^{1}$ Results for specific diseases and impairments have been extensively reported. ${ }^{2-46}$ These results drew attention to the importance of disability from musculoskeletal disorders, mental and substance use disorders, and various other noncommunicable diseases. ${ }^{1}$ In developing countries, disorders such as anaemia and neglected tropical diseases remained important contributors to health loss. ${ }^{18,43,47}$ More generally, the analysis showed the global transition towards a rapid increase in YLDs due to global 
population growth and ageing, combined with little progress in reduction of age-specific YLD rates.

In view of the ambitious goal of the GBD 2010, to synthesise the global evidence for the country-age-sex-year prevalence of all major disorders, several specific estimates were critiqued. Specific data sources, modelling assumptions, and aspects of the general approach were challenged and there was widespread recognition that more and higher quality data could improve the estimates. ${ }^{48-52}$ Disability weights that were used to calculate YLDs were based on surveys of the general public in five countries (Bangladesh, Indonesia, Peru, Tanzania, and the USA) and an open internet survey. The validity of disability weights was questioned for selected states including hearing loss, vision loss, drug use, spinal cord lesion, intellectual disability, and musculoskeletal disorders. ${ }^{53,54}$ Some investigators questioned whether disability weights should be used to measure health or the loss of wellbeing associated with health states. ${ }^{53,55}$ Additionally, the YLD uncertainty intervals were large for several disorders because of scarce data, hence there was a need to statistically adjust for different case definitions, measurement methods, and wide uncertainty intervals for disability weights. Wide uncertainty intervals reduced the number of significant differences for some disorders reported across time and countries. Broad interest and crucial discourse about GBD also drew attention to many unpublished data sources in specific countries that could be used to strengthen the analysis.

With the prominent role attached to quantification of disease burden for health research and policy nationally and globally, up-to-date estimates based on the latest evidence for descriptive epidemiology constituted an essential global public good. ${ }^{22-27,32,56-60}$ The GBD 2013 provides an opportunity to incorporate constructive criticism about GBD 2010 data sources, model development, methods, and interpretation. Additionally, the GBD 2013 shows methodological advances and includes new data for disability weights, capturing many new published or unpublished data sources for the disorders included in the GBD. Here, we report data, methods, and results from the analysis of 188 countries for 1990 to 2013 for 301 diseases and injuries and their 2337 sequelae. We report incidence for acute sequelae, prevalence for chronic sequelae, total prevalence by cause, in addition to YLDs for all causes. Because prevalence and YLDs for the entire period from 1990 to 2013 were reanalysed using consistent data and methods, these results supersede any previous publications about GBD.

\section{Methods}

\section{Overview}

Our general approach was similar to that for GBD 2010. The analysis of incidence and prevalence for HIV/AIDS, tuberculosis, and malaria for GBD 2013 have already been reported in detail. ${ }^{61}$ Key changes from GBD 2010 were the inclusion of new data through updated systematic reviews and the contribution of unpublished data sources from many collaborators; elaboration of the sequelae list to include asymptomatic states, such as Plasmodium falciparum parasitaemia (without symptoms); use of more detailed nature-ofinjury codes; improvements to the Bayesian meta-regression method; increased simulation 
size for comorbidity; estimation of the prevalence of injuries by cohort; and use of a novel method to estimate the distribution of mild, moderate, and severe anaemia by cause.

\section{Cause and sequelae list changes}

Based on feedback about GBD 2010, and input from the GBD 2013 collaborators, we expanded the cause and sequelae list (appendix pp 60-89). There were several key changes. First, we included asymptomatic states as explicit sequelae so that overall disease prevalence estimates were available, which might be useful for disease targeting, health service planning, or mass treatment strategies. Asymptomatic sequelae, by definition, were not associated with ill health and therefore were not assigned disability weights. Second, to deal with the challenge that some of the nature-of-injury categories used in the GBD 2010 were highly heterogeneous, these categories were expanded from 23 to 47 . Third, we added several new causes and sequelae. All these additions to the cause list were done to either reduce the size of the large residual categories, such as other injuries, or recognition of substantial epidemiological heterogeneity within a disease category (appendix pp 60-89). With these changes, the cause list was expanded from 289 to 301 causes and from 1160 to 2337 sequelae. Most of the increase in sequelae was due to the expansion of the nature-ofinjury sequelae, which applied to each of the external causes of injuries. The appendix pp 90-96 provides a list of the International Statistical Classification of Diseases and Related Health Problems, Tenth Revision (ICD-10) and International Classification of Diseases, Ninth Revision (ICD-9) codes for all GBD causes and the nature-of-injury categories.

\section{Data sources}

GBD 2010 collaborators undertook systematic reviews for most of the causes and sequelae. For some sequelae, the majority of the data came from household survey microdata reanalysis and administrative data such as hospital discharges. For others, most of the data were extracted from publications. Documentation of the GBD 2010 systematic reviews, however, was not centralised and only some of these reviews have been published. For this study, we updated systematic reviews through Aug 31, 2013. In some cases, studies published after Aug 31, 2013, were identified and included on the basis of GBD collaborator input; no data or studies were extracted after Nov 30, 2014. Household surveys including the demographic and health surveys, multiple indicator cluster surveys, living standards measurement surveys, reproductive health surveys, and various national health surveys included in the Global Health Data Exchange were systematically screened for data relevant to sequelae. For some diseases, case notifications reported to WHO were used as inputs and updated until the end of 2013. The appendix pp 97-653 provides a full list of citations for sources organised by country that were used for this analysis.

We computed an index of the geographical and temporal representativeness of the data sources available for non-fatal health outcomes for each cause or impairment - the data representativeness index (DRI). The overall DRI simply counts the fraction of countries that have any incidence, prevalence, remission, or excess mortality data available for causes that are prevalent in that country. We did not count cause of death data in this measure, even if it was used in the estimation of incidence or prevalence. We computed the same measure for three periods: before 1998, 1998-2005, and 2006 onwards. Table 1 provides the overall DRI 
and period-specific DRI measures for each cause and table 2 provides the same information for estimation of total impairment prevalence. The DRI was also computed for level 1 and level 2 causes (aggregate causes; see appendix pp 60-89) by counting data availability for any cause within that aggregate. This metric represents the availability of data and does not incorporate any assessment of data quality. The all-cause DRI was $100 \%$ overall and for each period, indicating that there was at least data for one cause for all 188 countries in each period. At more detailed levels, however, there was wide variation in the DRI across causes and time. DRI ranged from less than $2 \%$ for eight causes, including glucose-6-phosphate dehydrogenase deficiency trait and other mental and substance use disorders, to $100 \%$ for Chagas disease, African trypanosomiasis, and food-borne trematodiases. Causes with required infectious disease case reporting had high DRI values. Other disorders, such as cancers, had DRI values above $70 \%$ due to the network of population-based cancer registries. Although the time trend varied by disease, many of the highest DRI values were from 1998 to 2005. The lag in data analyses and publications might explain lower DRI values for 2006 to present.

Data representativeness can also be assessed at the country level. Figure 1 shows a map of the percentages of causes for which there were data available in each of the 188 countries between 1990 and 2013. The DRI values ranged from 6\% in South Sudan to 92\% in the USA. Many developed countries had data for more than $65 \%$ of causes; Brazil, India, and China have similar levels. Low levels of data availability were noted in several sub-Saharan African countries, central Asia, the Caribbean, and the Balkans. There was substantial variation within regions; for example, Kenya had 49\%, whereas Djibouti had less than 10\%, Laos had 14\%, and Thailand had 54\%.

\section{Sequelae incidence and prevalence}

The appendix pp 654-84 provides a brief description of the modelling strategy used for each sequela and cause. The most extensively used estimation method was the Bayesian metaregression method DisMod-MR 2.0. For some causes such as HIV or hepatitis B and C, disease-specific natural history models were used in which the underlying three state model in DisMod-MR 2.0 (susceptible, cases, or dead) was insufficient to capture the complexity of the disease process. For some diseases with a range of sequelae differentiated by severity, such as chronic obstructive pulmonary disease (COPD) or diabetes mellitus, DisMod-MR 2.0 was used to meta-analyse the data for overall prevalence. Separate DisMod-MR 2.0 models were then used to analyse data for the proportion of cases with different severity levels or sequelae. Likewise, DisMod-MR 2.0 was used to meta-analyse data for the proportions of liver cancer and cirrhosis due to underlying causes such as hepatitis B, hepatitis $\mathrm{C}$, and alcohol use. For acute sequelae, we report incidence (defined as a duration of 3 months or less) at the cause level in table 3, because incidence is the preferred measure for disorders of short duration.

DisMod-MR 2.0 represents a major advance in the computational speed, geographical disaggregation of full internally consistent posterior estimation, and display of data results compared with DisMod-MR 1.0, which was used in GBD 2010. Through cross-validation tests, Flaxman and colleagues reported ${ }^{62}$ that the log-rates specification of models worked 
as well or better than the negative binomial specification used in DisMod-MR 1.0. Based on these findings, and the substantial improvements in computational speed for log-rate models, this specification was the default method for DisMod-MR 2.0. The appendix pp 3-6 provides details of the DisMod-MR 2.0 likelihood estimation. The DisMod-MR 1.0 sequence of global estimation, regional estimation, and country prediction, which we call an analytical cascade, is illustrated in the appendix p 734. DisMod-MR 2.0 uses a more complete cascade (appendix p 735). At the global level, a mixed-effects non-linear regression with all available country data was used to generate initial global estimates that are passed to the next level of the DisMod cascade to inform the model for each super region. In turn, a super-region specific mixed-effects non-linear regression was used to estimate for regions. The same regression method was used for estimation of further geographical dis aggregation. The analyst could choose, depending on data density, to branch the cascade in terms of time and sex at different levels. In GBD 2010, DisMod-MR 1.0 was used to generate fits for three periods only: 1990, 2005, and 2010 because of long computational time. For GBD 2013, we generated fits for 1990, 1995, 2000, 2005, 2010, and 2013.

DisMod-MR 2.0 internal validity was assessed by use of $R^{2}$ for adjusted data. Results for all DisMod-MR 2.0 models are provided in the appendix pp 654-84. Adjusted data were the original study data transformed to the reference case definition and measurement method, using the meta-regression component of DisMod-MR 2.0 to make the data from different studies with varying methods comparable. External validity was also evaluated through cross-validation on a small number of sequelae due to the computational time and complexity for this analysis. We selected ten DisMod-MR 2.0 models representing a range of data densities to evaluate. We held out $30 \%$ of datapoints for incidence and prevalence at random, refit the model, and compared predictions to the held-out data. We assessed model performance using two metrics: the root-mean squared error of the predictions compared with the data held out, and the coverage of the data prediction with $95 \%$ uncertainty intervals. The appendix pp 736-37 provides these metrics for the ten models tested. In all cases, external validity was equal to or only slightly worse than the internal validity.

As in GBD 2010, DisMod-MR was not used to model estimates for a shortlist of causes; custom models were created for many of these. For some of these causes, important improvements in the modelling strategy were implemented. Changes for HIV and malaria have been described elsewhere. ${ }^{61}$ For dengue, the model was modified to use the first component of a principal components' analysis of Bhatt and colleagues' dengue transmission probability to improve estimation of case rates. ${ }^{63}$ For lymphatic filariasis, precontrol levels were estimated from data reported in the lymphatic filariasis atlas. ${ }^{64}$ Last, based on crucial input from GBD collaborators, we chose to model rheumatic heart disease in low-income and middle-income countries separately from high-income countries in view of potential differences in long-term cohort effects of treatment.

Estimation for cancer in GBD 2013 largely followed a similar analytical strategy to GBD 2010, which used a combination of incidence data, survival data, and sequelae durations to estimate cancer prevalence and YLDs. ${ }^{65,66}$ The analysis benefited from the inclusion of both the latest edition of Cancer Incidence in Five Continents and a larger number of other cancer 
registries particularly in China. In GBD 2013, we also incorporated new data from the US National Cancer Institute's Surveillance, Epidemiology, and End Results Program (SEER) ${ }^{67}$ and WHO's International Agency for Research on Cancer's Cancer Survival in Africa, Asia, the Caribbean, and Central America to update best and worst case survival, yearly survival trends, and sequelae durations for all cancers. ${ }^{68}$ Based on evidence that individuals with most cancers continue to have higher mortality beyond 5 years than do the general population, we estimated the burden of cancer for up to 10 years after incidence. Estimates for cancer sequelae now represent the burden for all cancer patients by contrast with estimation of the burden just for cancer survivors (see appendix pp 7-8 for more detail on aspects of estimating non-fatal cancer outcomes that were different from the methods used in GBD 2010).

\section{Injuries}

We followed a similar strategy to GBD 2010 for estimating the burden of injuries, except for an expanded list of 26 external cause-of-injury categories (from 15) and 47 nature-of-injury categories (from 23) for both short-term outcomes and lasting disability (see appendix pp 90-96 for ICD codes). More detail was added to both external causes and nature-of-injury categories to reduce epidemiological heterogeneity within each combination of cause and nature-of-injury category. The key analytical steps are explained in greater detail in the appendix pp 9-14. Here we provide a summary of the methods.

First, for each external cause, DisMod-MR 2.0 was used to analyse incidence based on hospital, emergency department, and survey data. Second, we estimated the distribution of nature of injury for each external cause using data that had both types of code available. When individuals were coded with more than one nature-of-injury code, we used the most severe. Third, we analysed seven studies that provided at least 1 year of follow-up for various natures of injury to estimate long-term disability. ${ }^{69-75}$ Fourth, we estimated cohort prevalence of long-term disability from the incident cases of injury for each external cause and nature-of-injury combination while accounting for excess mortality for the more severe post-injury sequelae. For some injuries, treatment modifies the disability weight. In these cases, we approximated the fraction of injuries receiving treatment as a function of an indicator of health system access. ${ }^{76}$

Short-term disability was estimated for all natures of injury by cause-of-injury categories as the product of prevalence (estimated by multiplying incidence by mean duration) and the appropriate disability weight. The duration for treated cases of injuries was determined by information in the Dutch Injury Surveillance System follow-up studies of 2001-04 and $2007-10 .{ }^{71,73}$ We used expert opinion to estimate a multiplier for the duration of short-term disability from untreated injuries and used the estimates of access to care by country and year as we have described for the long-term disability.

\section{YLDs from 29 residual causes}

Despite expanding our list of causes and sequelae in GBD 2013, many diseases remain for which we do not explicitly model disease prevalence and YLDs. The GBD cause list is collectively exhaustive such that all sequelae with an ICD code are mapped to a cause group 
(appendix pp 90-96). Many less common sequelae are included in 29 of the residual categories. For 14 of these cause groupings, epidemiological data for incidence or prevalence are available so that they can be modelled as other causes have been modelledthis set includes meningitis, cirrhosis, liver cancer, pneumoconiosis, and chronic kidney disease due to other causes, other neoplasms, other cardiovascular and circulatory diseases, other drug use disorders, other mental and substance use disorders, other gynaecological diseases, other musculoskeletal disorders, other skin and subcutaneous diseases, age-related and other hearing loss, other vision loss, other sense organ diseases, and other oral disorders. For 12 residual categories (other intestinal infectious diseases, other neglected tropical diseases, other maternal disorders, other neonatal disorders, other nutritional deficiencies, other infectious diseases, other chronic respiratory diseases, other digestive diseases, other neurological disorders, other urinary diseases, other haemoglobinopathies and haemolytic anaemias, and other congenital anomalies), epidemiological data for incidence and prevalence were not available for the entire residual cause groupings but sufficient cause of death data allowed for cause of death estimates. For each category, we identified causes within the larger cause group that had both estimates of years of life lost (YLLs) and YLDs, which we expected to have similar ratios of mortality to morbidity. We then computed the ratio of YLLs to YLDs for these specific causes (on a country-sex-year basis) and applied them to the residual category's YLLs to estimate its YLDs. This approach makes the simplified assumption that on average within a level 2 disease grouping the disability is proportionate to mortality within a country-sex-year. For an additional three residual categories (other sexually transmitted diseases, other drug use disorders, and other mental and substance use disorders), there were no overall epidemiological data or sufficient deaths to generate cause of death estimates. For the last two, we used US outpatient data or prevalence data from the Medical Expenditure Panel Survey (MEPS), National Epidemiologic Survey on Alcohol and Related Conditions (NESARC), or the 1997 Australian mental health survey ${ }^{77}$ and applied a severity distribution from these surveys in all countries and periods. These two causes for which US and Australian data were applied worldwide account for $1.6 \%$ of global YLDs.

\section{Impairments}

As in GBD 2010, we estimated the country-age-sex-year prevalence of nine impairments: anaemia, epilepsy, hearing loss, heart failure, intellectual disability, infertility, vision loss, Guillain-Barré, and pelvic inflammatory disease. These impairments were selected because they are sequelae of more than one disease and data are available to estimate prevalence for the overall impairment. Generally, overall impairment prevalence was estimated using DisMod-MR 2.0. Cause-specific estimates of impairments, such as the 19 causes of blindness, are required to provide the total prevalence estimated for that impairment. Anaemia, epilepsy, hearing loss, heart failure, intellectual disability, and pelvic inflammatory disease are estimated for different levels of severity. Separate estimates were made for primary infertility (in couples who have not been able to conceive) and secondary infertility (in couples having trouble conceiving again) and, for each, if the impairment is affecting men or women, or both. The severity distribution of cause-specific prevalence of each impairment was estimated as explained above or, in the absence of severity-specific data, assumed to be proportionate across all levels of severity. In the case of epilepsy, 
severity levels were determined by mixed-effect models for the proportions of primary, severe, and treated epilepsy, and a meta-analysis for seizure-free treated epilepsy, and thus had values that were specific for country, age, sex, and year. DisMod-MR 2.0 models produced country-specific, age-specific, sex-specific, and year-specific levels of hearing loss and vision loss. Due to little information about the severity levels of intellectual disability, we assumed a similar distribution of severity worldwide based on meta-analysis of intelligence quotient (IQ)-specific data for the overall impairment. This was supplemented with cause-specific distributions for chromosomal causes and iodine deficiency, whereas the severity of intellectual disability included in the long-term sequelae of causes such as meningitis, neonatal tetanus, and malaria was combined with several impairments such as motor impairment, blindness, or seizures. The severity of heart failure is derived from our MEPS analysis and therefore is not specific for country, year, age, or sex.

Our method for estimating overall anaemia was largely the same as in GBD 2010 but with the addition of new data sources, specifically subnational data for the UK, China, and Mexico. ${ }^{43}$ We adopted different thresholds for defining anaemia during the neonatal period, because the GBD 2010 thresholds did not account for haematological realities of early life. The GBD 2013 thresholds match the WHO recommendations ${ }^{78}$ with the exception of thresholds of less than 1 month because there is no international cutoff for diagnosis at that age. ${ }^{43,79}$ To disaggregate marginal estimates of anaemia severity and cause into a complete set of prevalence estimates for cause and severity pairs, we developed a new method for GBD 2013 that used techniques from Bayesian contingency table modelling. ${ }^{80,81}$

In GBD 2010, hearing loss of more than or equal to $35 \mathrm{~dB}$ in DisMod-MR 1.0 was estimated and then broken down into six severity levels based on a series of regressions on the proportionate distribution across categories. In GBD 2013, we first estimated the prevalence of normal hearing, hearing loss of 20-34 dB (mild), and greater than $35 \mathrm{~dB}$ (moderate and above); these three categories were fixed to add up to $100 \%$. We then ran separate DisModMR 2.0 models for five severity levels (ie, moderate 35-49 dB, moderately severe 50-64 $\mathrm{dB}$, severe 65-79 dB, profound 80-94 dB, and complete $\geq 95 \mathrm{~dB}$ ), which were then proportionally rescaled to fit in the $35 \mathrm{~dB}$ or greater envelope. In GBD 2010, the same severity distribution was assumed for each cause of hearing loss. In GBD 2013, we customised the prevalence estimation for each cause. Hearing loss due to otitis media and age-related hearing loss were estimated by DisMod-MR 2.0 using prevalence data. Hearing loss due to meningitis was estimated as a proportion of meningitis cases from a metaanalysis. ${ }^{82}$ Congenital hearing loss was estimated using birth prevalence data in DisModMR 2.0, assuming a constant prevalence for all ages because there was no evidence of an increased mortality risk. We assumed all hearing loss from otitis media was mild or moderate on the basis of reported distribution of hearing loss. ${ }^{83,84}$ To account for hearing aids, we assumed that the use of a hearing aid reduces the severity of hearing loss by one severity level. The other causes were assumed to cover the full range of severities. More details about impairments are provided in the appendix pp 15-32. 


\section{Severity distributions}

For 213 causes, a range of sequelae are defined in terms of severity. Important changes to the sequelae list with regards to severity include low back pain, alcohol and drug dependence categories, uterine prolapse, and epilepsy. Milder states for low back pain and alcohol and drug dependence categories were added because these disorders had a large gap between asymptomatic cases and the high value of the disability weight for the least severe symptomatic categories, whereas the epidemiological data for severity indicates a sizeable proportion of cases with milder disability. Stress incontinence was added as a sequela of uterine prolapse with a new disability weight that is distinct from full incontinence. Also, epilepsy health states are now better aligned with epidemiological data based on seizure frequency. In cases in which severity is related to a particular impairment, such as mild, moderate, and severe anaemia due to malaria, the analysis is driven by the impairment estimation described above. For some outcomes such as COPD or asthma, data have been gathered in different locations around the world and these have been modelled using DisMod-MR 2.0 (see appendix pp 694-733 for details). In other cases, published metaanalyses have been used to estimate the allocation of cases by severity. For the remaining causes, we used the same approach for estimating the distribution of severity as in the GBD 2010; empirical analysis of this model was updated through the addition of 2 years from the US MEPS. The appendix pp 685-87 lists the GBD causes that can be identified in MEPS and the corresponding ICD-9 CM codes. In total, 203960 observations, covering 119676 individuals, were used. In the cases of dementia, Parkinson's disease, multiple sclerosis, osteoarthritis, schizophrenia, and bipolar disorder, data identified through literature reviews were used to inform the severity distribution. The introduction of a mild health state for four drug dependence categories required identification of epidemiological data to estimate the proportion of cases with mild versus more severe disability. For cannabis dependence, we used the NESARC survey in the USA and the Australian National Survey of Mental Health and Wellbeing. For the remaining three drug dependence categories, we only had access to one study on polydrug users in Australia, which led to about half of dependent cases being assigned to the more severe and mild health states. Although this information is derived from a non-representative cohort of drug users, it was thought to be more appropriate than deriving a severity distribution from a household survey like NESARC in which only a small proportion of individuals dependent on opioids, cocaine, or amphetamines would be represented.

\section{Revisions to disability weights}

The GBD 2010 disability weights measurement study introduced a new method of pairwise comparisons as a means of eliciting weightings for health states in population surveys. ${ }^{85,86}$ Data were gathered in five countries (Bangladesh, Indonesia, Peru, Tanzania, and the USA) and supplemented with a web survey. In total, responses were gathered from 30230 people in 167 countries. Respondents were presented with a series of randomly selected pairwise comparisons of lay descriptions of health states and asked to state which health state is healthier than the other. Salomon and colleagues ${ }^{85}$ developed a statistical model that yields from these pairwise comparisons disability weights on a scale from 0 (no health loss) to 1 (equivalent to death). 
Based on important commentary and review of the GBD 2013 collaborators, we have revised the lay descriptions of 32 states and added 16 new states. The revised lay descriptions were based on identifying inconsistency in the way progression across levels of severity had been handled for some outcomes and the addition of social isolation to the descriptions for complete, profound, and severe hearing loss. New states included five milder health states for alcohol and drug dependence; two health states for the alignment of epilepsy with the epidemiological data defining severe epilepsy in individuals who had on average one or more fits per month and less severe epilepsy in those with between one and 11 fits in the past year; two milder health states for low back pain; and one each for stress incontinence, concussion, hypothyroidism, hyperthyroidism, thrombocytopenic purpura, vertigo, and amputation of one arm without treatment. The appendix pp 688-93 provides a complete list of the lay descriptions of all 235 GBD 2013 health states.

In 2013, we had the opportunity to collaborate with the European Centre for Disease Prevention and Control to gather new data for disability weights in four population-based national surveys (Hungary, Italy, Sweden, and the Netherlands) using the Salomon and colleagues' protocol ${ }^{85,87}$ Because of funding and questionnaire length, the surveys included 140 of 220 GBD 2010 health states for which the lay descriptions had not been revised, 32 health states with revised lay descriptions, and 42 new health states, 16 of which were included in GBD 2013. These nationally representative samples were comprised of 30660 respondents. For GBD 2013, the data of GBD 2010 disability weights measurement study and the European disability weights measurement study ${ }^{88}$ were pooled in a single analysis of individual responses, thus doubling the number of respondents to 60890 in both studies. For states where the lay description was not previously included, revised, or new, only the European disability weights measurement study data were used. This means that all disability weights in GBD 2013 differ from the GBD 2010 disability weights. Most disability weights changed slightly, but some differ more widely (appendix pp 688-93). Some of the more substantial changes were due to the inclusion of incontinence in the lay descriptions for spinal cord injury and the inclusion of the psychological consequences of social isolation in people with more severe hearing loss, leading to much higher disability weights. The statistical analysis generates uncertainty distributions for each disability weight that are propagated into the uncertainty distributions of the estimates of YLDs.

\section{Comorbidity}

Many individuals have more than one disease or injury sequela at the same time. To accurately account for comorbidity and its effect on disability for individuals, we used the GBD 2010 microsimulation approach. In the microsimulations, a set of individuals are exposed to the probability of having all the different sequelae included in the GBD to estimate a distribution of the combinations that might be seen in each country-age-sexyear. We modelled the probabilities within each country-age-sex-year of different sequelae as independent. Although there are clear examples of the probability of one sequela changing the probability of other sequelae, such as diabetes and ischaemic heart disease, testing reported by Vos and colleagues ${ }^{1}$ suggested that modelling assuming independence was a reasonable approximation. However, for less common sequelae the microsimulation tends to increase the estimated uncertainty in the number of YLDs substantially because, for 
example, a sequela that is estimated to have a prevalence of less than one in 10000 will not appear randomly in many microsimulations of 20000 . Two steps have been taken to reduce the inflation of uncertainty for uncommon sequelae. First, the number of simulants in each country-age-sex-year was increased to 40000 ; the main limiting factor for the number of simulants is computational resources needed to run each of the 62880 country-age-sexyear simulations 1000 times to account for uncertainty in each of the input prevalence rates. Second, we excluded sequelae in a country-age-sex-year with a prevalence of less than one in 20000 from the microsimulation. The combined disability weight for individuals with several sequelae was computed as in the GBD 2010 using a multiplicative model; namely, the individual's disability weight is equal to one minus the cross product of one minus the disability weight for each sequela that the individual has. An output from the comorbidity microsimulation is counts of the number of sequelae for each simulant in the population. The numbers of simulants with different comorbidities in a country-age-sex-year was adjusted from 40000 to equal the estimated population in each country-age-sex-year to produce the estimated distribution of individuals in each country with comorbidities. Sequelae with a prevalence of less than one in 20000 that were not included in the microsimulation, are also not included in the population pyramids showing individuals by numbers of sequelae (figure $2 \mathrm{~A}-\mathrm{C}$ ). A technical description of the comorbidity simulation is given in the appendix $\mathrm{p} 2$.

We have reported $95 \%$ uncertainty intervals for each quantity in this analysis. For disease or sequelae incidence or prevalence rates, age-standardised rates or counts, the models such as DisMod-MR 2.0 provide posterior distributions for each quantity from which $95 \%$ uncertainty intervals are computed. For YLDs, we incorporated uncertainty in prevalence and uncertainty in the disability weight into the posterior distribution of YLDs. In practice, we estimated the posterior distribution of YLDs by taking 1000 samples from the posterior distribution of prevalence and 1000 samples of the disability weight to generate 1000 samples of the YLD distribution. We estimated the $95 \%$ uncertainty interval by reporting the 25th and 975th values of the distribution. Uncertainty intervals for YLDs at different timepoints $(1990,1995,2000,2005,2010$, and 2013) for a particular disease or sequela are correlated because of the shared uncertainty in the disability weight.

\section{Role of the funding source}

The funder of the study had no role in study design, data collection, data analysis, data interpretation, or writing of the report. The corresponding author had full access to all the data in the study and had final responsibility for the decision to submit for publication.

\section{Results}

Figure $2 \mathrm{~A}-\mathrm{C}$ shows the population pyramid for developed countries, developing countries excluding sub-Saharan Africa, and sub-Saharan Africa in 1990 and 2013 broken down by the number of sequelae, ranging from none to more than ten sequelae. Most of the world's population had at least one of the GBD sequelae and most people had several. As expected, in view of the strong relation between age and disease prevalence for most noncommunicable diseases and injuries, the number of individuals with several morbidities 
rapidly increased with age. In developed countries in $2013,35.9 \%$ of the age group $0-4$ years had no sequelae with only $0.03 \%$ older than 80 years with no sequelae (figure $2 \mathrm{~A}$ ). In the age group older than 80 years, $10 \cdot 3 \%$ had one to four sequelae, $64.6 \%$ had five to nine sequelae and $25.1 \%$ had ten or more sequelae in 2013. The percentage of each age group with several morbidities rose progressively with age irrespective of the cutoff used to define several morbidities. Due to this relation and the demographic shifts towards older ages in developed countries, the number of individuals with more than ten sequelae increased by $51.6 \%$ from 1990 to 2013. In the oldest age group, $23.6 \%$ of women and $27.8 \%$ of men had more than ten sequelae, but the large population imbalance at older age favouring women meant that there were 1.4 times more women than men with ten or more sequelae.

Figure 2B shows the pyramids for developing countries outside of sub-Saharan Africa, showing that the birth cohorts in 2013 were smaller than in 1990. The major demographic change was the large expansion of adults in the age groups 20-54 years for men and women from 1990 to 2013. Comparison of 1990 and 2013 showed little change in the distribution of the population in each age group by the number of sequelae. Rising numbers with several morbidities were attributable to ageing. $20.3 \%$ of the age group $0-4$ years and $0.05 \%$ older than 80 years had no sequelae. In the oldest age group, $12.5 \%$ had one to four sequelae, $63.9 \%$ had five to nine sequelae, and $23.5 \%$ had ten or more sequelae.

As shown in figure $2 \mathrm{C}$, the main result was the massive growth in population in sub-Saharan African countries from 1990 to 2013 and continued pattern of a low percentage of the population at older ages driven by high fertility and high mortality. Due to several very common sequelae that start early in life such as anaemia, soil-transmitted helminths, and schistosomiasis, only $7.8 \%$ of the age group $0-4$ years and $0.002 \%$ at age 80 years and older in sub-Saharan Africa had no sequelae in 2013. Although there were few individuals reaching the oldest age groups, in the population aged 80 years and older, $1.9 \%$ had one to four sequelae, $42.6 \%$ had five to nine, and $55.5 \%$ had ten or more sequelae, which were higher than in developed countries. Multiple morbidities were also common in all regions in working age adults (20-64 years): $31.7 \%$ with five or more sequelae in developed countries, $37.9 \%$ in developing countries outside of sub-Saharan Africa, and 61.6\% in sub-Saharan Africa. Of the 2.3 billion individuals in 2013 with more than five sequelae, $81.4 \%$ were younger than 65 years.

The comprehensive and systematic nature of GBD provides an opportunity to assess the most common acute disorders affecting people around the world and the trends from 1990 to 2013. Table 3 shows the 65 causes of acute disease ( $<3$ months' duration) and injury incidence with more than 1 million cases per year in 2013. For the causes with more than one acute sequela, such as typhoid fever (acute infection, intestinal perforation, and intestinal bleeding), maternal sepsis (puerperal sepsis and other maternal infections), or hypertension in pregnancy (eclampsia, pre-eclampsia, and other hypertensive disorders in pregnancy), we aggregated all the acute sequelae for a cause for presentation in table 3 . Of note, there are two disorders with an incidence of greater than 2 billion in 2013: upper respiratory infections ( 18.8 billion) and diarrhoeal diseases ( $2 \cdot 7$ billion). Another 12 diseases and injuries accounted for between 100 million to 1 billion incident cases per year in 2013: injuries due to other exposure to mechanical force, acute otitis media, tooth pain due to 
caries of permanent teeth, bacterial skin diseases (including impetigo and abscess), falls; lower respiratory infections, clinical episodes of malaria, chlamydia infection, varicella (including chickenpox and herpes zoster episodes), acute hepatitis B, gallbladder and biliary tract disease, and acute hepatitis A. There were 28 diseases and injuries with incident cases between 10 million and 100 million per year including several injuries, such as nonvenomous animal contact, motor vehicle road injuries, fire, heat, and hot substances, motorcycle and pedestrian injuries, and infections such as urinary tract infections, typhoid, hepatitis $\mathrm{C}$ and $\mathrm{E}$, dengue, gonorrhoea, the initial episodes of genital herpes, trichomoniasis, and several disorders affecting the digestive system, including gastritis and duodenitis, peptic ulcer disease, pancreatitis, and appendicitis.

Among the most common causes of acute disease incidence, 47 increased in absolute numbers of incident cases from 1990 to 2013 but only 13 had rising age-standardised rates of which six had significant increases (upper respiratory infections, interstitial nephritis, urinary tract infections, dengue, pancreatitis, paralytic ileus, intestinal obstruction, and unintentional suffocation; table 3). Numbers declined for 18 of 65 causes in table 3 , seven of which were infectious diseases that predominantly affected children. Two were maternal disorders and for one, peptic ulcer disease, the decline was not significant. Some injuries such as non-venomous animal contact, fire, heat, and hot substances, collective violence and legal intervention, exposure to forces of nature, venomous animal contact, poisoning, and drowning decreased in absolute incidence numbers. For 52 of 65 causes, age-standardised rates declined-for 34 causes, numbers increased due to demographic change even though age-standardised rates declined. In the set of causes of more than 1 million cases per year, dengue had the most striking increase in the age-standardised rates (447.3\%; table 3).

Table 4 summarises the prevalence of chronic disease and injury sequelae ( $>3$ months) aggregated to the cause level. This table provides a high-level view of the leading causes of chronic disorders worldwide for the 59 causes with a global prevalence of greater than $1 \%$ in 2013. Leading causes were a mixture of oral disorders, neurological disorders, skin diseases, musculoskeletal disorders, neglected tropical diseases, gynaecological disorders, chronic kidney disease, some causes of anaemia, age-related hearing, other vision loss, and injuries. Eight causes affected more than 10\% of the world population in 2013: permanent caries without pain, tension-type headaches, iron-deficiency anaemia, glucose-6-phosphate dehydrogenase deficiency trait, age-related and other hearing loss, genital herpes without symptoms, migraine, and ascariasis. Another 51 causes afflicted between $1 \%$ and $10 \%$ of the world's population. In this set of disorders were those that were not prominent causes of YLDs because the average disability weight was low, but they might have been important in terms of health system resources or health service planning. These disorders included glucose-6-phosphate dehydrogenase deficiency, genital prolapse, premenstrual syndrome, edentulism, polycystic ovary syndrome, uterine fibroids, and several skin diseases.

Rates for only six causes of chronic disease (micronutrient deficiencies, worm infestations, and chronic hepatitis B; table 4) declined fast enough to lead to declines in the absolute numbers for each worldwide. As noted in table 3, several acute infectious diseases and other acute disorders also declined in absolute numbers. The numbers of cases increased for 17 other chronic disorders but there were significant reductions in age-standardised rates: age- 
related and other hearing loss, genital herpes, uncorrected refractive error, deciduous caries without pain, dermatitis, edentulism and severe tooth loss, osteoarthritis, uterine fibroids, chronic hepatitis $\mathrm{C}$, viral skin diseases, chronic kidney disease due to hypertension, ischaemic heart disease (angina, post-myocardial infarction, and heart failure), alcohol use disorders, asthma, chronic kidney disease due to glomerulonephritis, injury due to other exposure to mechanical forces, and endocrine, metabolic, blood, and immune disorders. Five chronic disease and injury states showed significant increases in age-standardised rates of greater than 5\% from 1990 to 2013: glucose-6-phosphate dehydrogenase deficiency, diabetes mellitus, sickle cell trait, other musculoskeletal disorders, and urolithiasis.

Table 5 show the estimated prevalence and the distribution of prevalence by cause and severity of five major impairments in 1990 and 2013 (the remaining four impairments are reported in the appendix pp 15-32). Table 5 also provides overall YLDs due to each impairment to provide context for the total burden related to each impairment.

We estimated that 1.83 billion individuals had anaemia in 1990 , rising to 1.93 billion in 2013 (table 5). Taking into account the distribution of anaemia across mild, moderate, and severe, total anaemia YLDs equalled 62.0 million in 1990, dropping slightly to 61.5 million in 2013. This number of YLDs made anaemia from all causes larger than the second leading disease contributing to YLDs, major depressive disorder (table 10). In addition to the reduction in overall prevalence, there was a small but notable shift towards more cases of mild anaemia and less severe and moderate anaemia from 1990 to 2013. By 2013, 49.2\% of individuals had mild anaemia, $46.9 \%$ had moderate anaemia, and $3.9 \%$ had severe anaemia (table 5). Iron-deficiency anaemia accounted for $62.6 \%$ of all cases and $31.5 \%$ of mild, $28.7 \%$ of moderate, and $2.4 \%$ of severe anaemia. The next five most common causes of anaemia overall were thalassaemia trait, malaria, gastritis, and duodenitis, other neglected tropical diseases, and other haemoglobinopathies and haemolytic anaemias. Hookworm and schistosomiasis together accounted for 55.2 million cases and malaria accounted for a further 80.6 million cases. From 1990 to 2013, the number of anaemia cases due to malaria increased by $38.9 \%$. Causes with an increase in cases of more than $50 \%$ included chronic kidney disease due to diabetes mellitus, chronic kidney disease due to other causes, and sickle cell disorders.

Table 5 shows that the estimated number of individuals with some form of hearing impairment ( $20 \mathrm{~dB}$ or more) rose from 807.2 million in 1990 to 1.23 billion in 2013. Globally, hearing loss accounted for $25 \cdot 1$ million YLDs in 1990, increasing $45 \cdot 3 \%$ to $36 \cdot 5$ million YLDs in 2013. In 2013, 800.7 million people had mild hearing loss (less than 35 $\mathrm{dB}$ ), whereas 414.5 million had moderate or moderate to severe hearing loss. The number with complete hearing loss ( $8 \cdot 0$ million) was somewhat higher than in 1990 (6.7 million). Just over 90\% of hearing loss was classified as age-related and other hearing loss in 2013. Otitis media was the next most important cause of overall hearing loss but only caused mild or moderate hearing loss. In 2013, congenital anomalies accounted for $2.1 \%$ of all hearing loss but $21 \cdot 1 \%$ of complete hearing loss.

Table 5 provides a detailed breakdown at the global level of the 61.7 million cases of heart failure (left-sided and right-sided) in the world, more than half of which were classed as 
severe. Worldwide, the number of individuals who had heart failure increased $96.4 \%$ from 1990 to 2013. A third of heart failure was due to ischaemic heart disease (table 5). Five other causes accounted for $62.0 \%$ of heart failure: hypertensive heart disease, other cardiovascular and circulatory diseases, cardiomyopathy and myocarditis, COPD, and rheumatic heart disease (table 5). All other causes each accounted for about 5\% of heart failure. Notably, in 2013, Chagas disease accounted for $0.6 \%$ of heart failure globally but $11.4 \%$ in Brazil. All causes of heart failure accounted for 8.6 million YLDs in 2013 (table 5).

Table 5 shows that the number of individuals with intellectual impairment increased from 118.2 million in 1990 to 154.0 million in 2013. In 2013, we estimated that borderline (IQ 70-84) and mild intellectual impairment (IQ 50-69) accounted for 104.9 million cases and moderate, severe, and profound intellectual impairment around 49.1 million cases. The most important causes of intellectual impairment in 2013 were idiopathic intellectual disability (61.5\%) followed by neonatal causes (mainly preterm birth complications and neonatal encephalopathy), congenital causes (mainly Down's syndrome and chromosomal unbalanced rearrangements), cerebrovascular disease, and infectious causes (mainly meningitis; tables 5-9). Comparison of the levels by cause in 1990 and 2013 showed that idiopathic intellectual disability increased $22.7 \%$, intellectual disability from preterm birth complications increased $129.6 \%$, and intellectual disability from neonatal encephalopathy decreased $5.8 \%$.

Table 5 shows the distribution of visual impairment including presbyopia in 1990 and 2013. The numbers of individuals with visual impairment, taking into account the availability of visual aids, increased from 518.6 million in 1990 to 774.1 million in $2013.72 .4 \%$ of this total in 2013 was uncorrected presbyopia. Excluding presbyopia, in 1990 there were $137 \cdot 0$ million individuals with moderate or severe vision loss increasing to 178.8 million in 2013 (table 5). Over the same period there was an increase in blindness from 23.1 million to $33 \cdot 0$ million. The increase in age-standardised rates per 100000 for visual impairment overall was from 12702.2 to 11740.9 and for blindness from 602.5 to 521.3 . The most important cause of visual impairment in terms of prevalence and YLDs was uncorrected refractive error accounting for just over $85 \%$ of all cases and 56\% of YLDs due to vision impairment. In terms of YLDs, the next most important cause was cataract followed by other vision loss, preterm birth complications, glaucoma, macular degeneration, and diabetes.

Figure 3 shows the comparison of the leading causes of global YLDs in 1990 and 2013, which provides detailed cause breakdowns that were most relevant to prioritising specific programmes or interventions. The top cause in 1990 and 2013 was low back pain. The second leading cause changed due to the decline in iron-deficiency anaemia and the rise in major depressive disorder. Two more top ten causes were musculoskeletal disorders: neck pain and the large category of other musculoskeletal disorders. Other top ten causes included migraine, age-related and other hearing loss, COPD, anxiety, and diabetes. Causes that increased more than two ranks from 1990 to 2013 included diabetes mellitus, osteoarthritis, dysthymia, medication overuse headache, and Alzheimer's disease and other dementias. Declines of more than two ranks were noted for dermatitis, diarrhoeal diseases, acne vulgaris, conduct disorder, and war and legal intervention. 
Table 10 provides the global estimates of prevalence and YLDs for 2013 and change from 1990 to 2013 for each cause (the full details at the levels of the sequelae; prevalence, YLD, and change between 1990 and 2013 by cause and age group; and prevalence, YLD, and change between 1990 and 2013 by cause and country are shown in the appendix pp 738811). In the GBD framework, individuals should be assigned to a unique sequela such that the sum of the YLDs or prevalence by sequela should equal the total prevalence and YLDs for a disease or injury. Since specific sequelae are of substantive interest to help target interventions or needs for new interventions, and to enhance the transparency of computation, we have provided the full list of causes and sequelae. Comparison of the percentage change in absolute number of YLDs from 1990 to 2013 and the percentage change in the age-standardised YLD rate shows where demographic change, both population increase and rising mean age, had a major effect. Age-standardised YLDs and prevalence for several infectious diseases showed significant declines of greater than $10 \%$ including diarrhoeal diseases, typhoid, paratyphoid, lower respiratory infections, meningitis, encephalitis, diphtheria, whooping cough, tetanus, measles, Chagas, African trypanosomiasis, cysticercosis, cystic echinococcosis, lymphatic filariasis, onchocerciasis, trachoma, rabies, ascariasis, trichuriasis, and hookworm. By contrast, significant increases in age-standardised rates of greater than 10\% were noted for HIV (from 1990 to 2013, but declines since 2005) ${ }^{61}$ cutaneous and mucocutaneous leishmaniasis, and dengue. Some other disorders such as malaria, tuberculosis, upper respiratory infections, varicella, and schistosomiasis did not show significant changes in either direction of greater than $10 \%$.

Age-standardised YLD rates for all maternal causes and sequelae combined declined significantly. Overall age-standardised YLDs for neonatal disorders increased 80.5\% from 1990 to 2013 (table 10). Large increases in age-standardised rates were noted for all neonatal causes. Age-standardised YLDs and prevalence of nutritional deficiencies decreased as did the absolute numbers of cases. Although there was a decline in the agestandardised rates for syphilis, they were either non-significant or small for chlamydia and gonorrhoea (table 10). The decline was significant for hepatitis $\mathrm{B}$ and $\mathrm{C}$, but non-significant for hepatitis $\mathrm{A}$ and $\mathrm{E}$. Of note, the number of individuals with hepatitis $\mathrm{C}$ infection increased.

Age-standardised YLD rates from all non-communicable diseases changed by only $1.4 \%$ from 1990 to 2013, but YLD numbers increased by 54.2\% (table 10). Stagnant overall rates masked highly diverse trends for specific causes. Overall, neoplasm YLDs increased 82.5\% and age-standardised rates increased significantly by $8.5 \%$. However, within the category of neoplasms, significant declines were noted for stomach cancer, liver cancer due to alcohol use, liver cancer due to other causes, larynx cancer, cervical cancer, nasopharynx cancer, gallbladder and biliary tract cancer, and bladder cancer. Significant increases were noted for liver cancer due to hepatitis $\mathrm{C}$, breast cancer, prostate cancer, colon and rectum cancer, pancreatic cancer, non-melanoma skin cancer, kidney cancer, thyroid cancer, non-Hodgkin lymphoma, and other neoplasms. Of note, there was no significant change in the trachea, bronchus, and lung cancer age-standardised rates. YLDs for cardiovascular diseases overall increased $89.2 \%$, but age-standardised rates did not change significantly. Among cardiovascular causes, age-standardised YLD rates declined significantly for atrial fibrillation and flutter and peripheral vascular diseases. By contrast, age-standardised rates 
increased significantly by more than 5\% for hypertensive heart disease and cardiomyopathy and myocarditis.

Numbers of YLDs from chronic respiratory diseases increased from 1990 to 2013 by $55 \cdot 1 \%$ but age-standardised rates remained stagnant (table 10). Overall cirrhosis age-standardised prevalence and YLD rates declined, although for cirrhosis caused by hepatitis $C$ the change was not significant. Among the digestive diseases, gastritis and duodenitis, appendicitis, inguinal, femoral, and abdominal hernia, gallbladder and biliary diseases, and other digestive diseases increased in absolute terms but declined in age-standardised rates. By contrast, inflammatory bowel diseases, paralytic ileus and intestinal obstruction, and pancreatitis increased in numbers and rates. YLDs from neurological disorders, as a group, increased 59.6\%, but age-standardised rates increased by only $5.0 \%$. There was no change in age-standardised rates for Alzheimer's and other dementias but YLD numbers increased by $91.8 \%$. The age-standardised rates of Parkinson's disease and primary epilepsy increased but not significantly, consistent with the consideration that they are largely genetically determined. YLDs from multiple sclerosis increased in age-standardised rates and numbers. It is not clear whether this increase in rates was due to improvements in case ascertainment or indicates a true increase in disease prevalence. Among the headache disorders, only medication overuse headache had a significant change in rates of $43 \cdot 3 \%$.

Overall, the YLDs for mental and substance use disorders increased 45.0\% from 1990 to 2013 , but there was only a $1.0 \%$ increase in the age-standardised rate (table 10). For all disorders in this group, including autism, Asperger's syndrome, anorexia nervosa, and bulimia, there were increases and decreases in age-standardised rates of less than $10 \%$. Diabetes mellitus YLDs increased by $135.7 \%$, but age-standardised rates increased only 43.4\%. By contrast, chronic kidney disease YLDs increased $49.5 \%$ but rates declined by $2.8 \%$. Patterns in age-standardised rates were different between specific causes of chronic kidney disease with an increase in chronic kidney disease due to diabetes but a reduction in chronic kidney disease due to hypertension and glomerulonephritis. Among urinary diseases, the increase in numbers and age-standardised rates for urolithiasis was notable. For most gynaecological diseases, numbers increased, but the changes in age-standardised rates were small; the category uterine fibroids was an exception with a reduced rate and only a small increase in numbers.

YLDs for the large category of musculoskeletal disorders increased by $60 \cdot 7 \%$, but there was no change in the age-standardised rate (table 10). The only exception to this general pattern was rheumatoid arthritis for which age-standardised rates declined significantly by $4.6 \%$. YLDs due to congenital disorders, as a group, increased $61.4 \%$ and age-standardised rates by $19.7 \%$. These large increases in numbers and age-standardised rates were noted for all congenital causes except Klinefelter's syndrome, for which the rate did not increase significantly. The reason for the large increases in prevalence and YLDs was the improved survival because birth prevalence for congenital disorders remained stable or declined (data not shown). Although skin diseases, as a group, did not show a significant pattern for agestandardised YLDs, some causes including cellulitis, bacterial skin diseases, and decubitus ulcer declined significantly. Most causes of vision and hearing loss included in the sense 
organ category increased in absolute numbers of YLDs but the age-standardised rates declined.

Injury age-standardised YLD rates decreased substantially from 1990 to 2013. There were reductions of more than $30 \%$ in age-standardised YLD rates for road injuries, drowning, fire, heat, and hot substances, poisoning, venomous animal contact, non-venomous animal contact, and self-harm (table 10).

The main disorders that drove changes in rates varied with age (appendix pp 776-811). In childhood, most infectious diseases and iron-deficiency anaemia showed decreases in YLD rates, whereas neonatal disorders and congenital disorders showed increases, largely due to lower initial case fatality and better long-term survival, and in the case of preterm birth complications an increase in the birth prevalence (data not shown). For ages 15-49 years, large increases from 1990 to 2013 were noted in disability associated with diabetes (appendix pp 776-811), HIV, and medication overuse headache, whereas there were large decreases in the YLD rates of iron-deficiency anaemia, falls, and collective violence. In adults aged 50-69 years, reductions in YLD rates for all injuries (appendix pp 776-811) and increase in diabetes YLD rates were notable.

Figure 4 shows the ten leading causes of YLDs for each country. Causes are colour coded by their global rank to emphasise where they vary substantially in the leading set. There was substantial consistency in the top causes. Low back pain and major depressive disorder were among the top ten causes of YLDs in every country. 40 causes were in the top ten list across all countries but only 16 of these causes were in the top ten of more than 20 countries. Low back pain was the leading cause in 45 of 50 developed countries and major depressive disorder was the leading cause in three countries, with neck pain and diabetes leading in one country each. Across developing country regions, there was more variation in leading causes. Low back pain or major depressive disorder was the leading cause in 94 of 138 developing countries. But other causes such as iron-deficiency anaemia, HIV, and war were leading causes in more than one country. Regional patterns emerged such as the more prominent role of falls in central Europe where they were ranked second in 11 of 13 countries. The category other musculoskeletal—which includes disorders such as shoulder problems, pathological fractures from osteoporosis, osteomyelitis, pyogenic arthritis, and systemic lupus erythematous-was prominent in high-income Asia and high-income North America. Anxiety disorders ranked more highly in many Caribbean nations and diabetes was prominent in Mexico, Nicaragua, Panama, and Venezuela. In Oceania, some soil-based helminths were highly prevalent, pushing them into the top five of the YLD rank list. Onchocerciasis, predominantly the onchocercal skin sequela, was ranked highly in Liberia, Cameroon, and South Sudan. The disability from past war and conflict ranked as the top cause of YLDs in Cambodia, Nicaragua, and Rwanda, and number two in Vietnam.

The figure appendix provides an analysis of the change in the YLDs per person from 1990 to 2013 by country and region. The countries were ordered by YLDs per person in 1990 . Across countries in 1990, YLDs per person ranged from 0.072 to more than double at 0.170. Generally, developed countries with higher mean age for the population had higher YLDs per person, but there were many exceptions to this pattern. Changes in the YLDs per person 
were decomposed into contributing causes for YLDs—-for convenience these cause groups were chosen to represent the major causes of YLDs at the global level. The figure provides a summary of what causes contributed to increases or decreases in YLDs per person for each country.

From 1990 to 2013, 139 of 188 countries had increases in the YLDs per person. Although the drivers of increased YLDs per person varied by region and country, generally, musculoskeletal, mental, substance use, neurological, and chronic respiratory disorders played important parts across most of the regions and countries. In sub-Saharan Africa, increases were largely driven by HIV/AIDS. Declining levels of iron-deficiency anaemia contributed to a decline in YLDs per person in many countries. In the subset of countries where the overall YLDs per person declined, specific factors such as long-term disability due to war were important factors-most strikingly evident in Lebanon. However, declines due to neglected tropical diseases and malaria, as well as diarrhoea and lower respiratory infections (represented as other communicable diseases in the figure appendix) also pushed YLD per person lower in some countries. Mostly increasing YLDs per person meant that by 2013 the range across countries was from 0.076 to 0.153. It is important to note that the figure appendix shows YLDs per person and not age-standardised YLD rates; therefore, changes in YLD rates were affected by changes in population age structure and changes in age-specific disease rates.

From 1990 to 2013, YLDs per person rose in most countries and YLLs per person declined. Both trends led to a shift towards greater disability as a share of the overall burden. Figure 5 shows the shift from 1990 to 2013 towards greater disability as the ratio of YLDs to YLDs plus YLLs ${ }^{76}$ (namely disability-adjusted life years [DALYs]). 30 countries as of 2013 had the most DALYs due to YLDs, representing a major change from historical patterns of premature mortality being the dominant set of health issues. In 1990, premature mortality still represented more than half of DALYs in every country. By 2013, the lowest YLD to DALY ratio outside of sub-Saharan Africa was in Afghanistan, but most developing countries outside of sub-Saharan Africa had ratios of greater than 35\%. In sub-Saharan Africa, however, the ratio of YLDs to DALYs ranged from 10.4\% in Mali to $38.9 \%$ in Cape Verde.

\section{Discussion}

We analysed more than 35620 epidemiological sources from 188 countries spanning the past three decades to provide the most up-to-date empirical assessment of the leading causes of acute disease incidence, chronic disease prevalence, and YLDs for 6 years (1990, 1995, 2000, 2005, 2010, and 2013) for 188 countries using consistent and comparable methods. Importantly, our study provides the first comprehensive assessment of the extent, pattern, and trend of non-fatal health loss in countries, with important implications not only for health policy, but also for the provision and financing of health services (panel).

A wide array of disease and injury sequelae affects the world's population. Globally, only $4.3 \%$ of the population had no burden of disease or injury sequelae in 2013, up slightly from $4.2 \%$ in 1990. There were 59 diseases and injuries with a global prevalence of greater than 
$1 \%$, but each caused little disability. These disorders comprised various causes of mild to moderate vision impairment, hearing loss, soil-transmitted helminths, mild anaemia, caries, and many others. For many of these common but fairly mild disorders, there are effective interventions. ${ }^{89-92}$ The GBD provides several insights into the health of different populations by quantifying the prevalence of a wide range of disorders and YLDs that take into account the general public's view of severity.

Of the 240 GBD causes that led to mortality, Naghavi and colleagues ${ }^{76}$ reported that global age-standardised death rates were declining by $80 \%$. For YLDs, the distribution of causes where age-standardised rates were declining, stagnant, or increasing was different. For 140 causes, distributed across communicable diseases, non-communicable diseases, and injuries, age-standardised rates declined significantly from 1990 to 2013. For 89 causes, changes in age-standardised rates were statistically indistinguishable from zero over the same period. For 72 causes, including epidemic disorders like HIV and dengue but also cancers, diabetes, and COPD, age-standardised rates increased significantly. Of 301 causes, the percentage change in the age-standardised rate for YLDs was higher than the percentage change in the age-standardised YLLs for 213 causes. Divergence in rates for diseases and injuries between mortality and morbidity could be because of reductions in case-fatality rates due to treatment or improved background risks such as malnutrition. To the extent that mortality is declining faster than disease prevalence due to treatment, access to care might be a crucial driver of trends in health. The mortality-disability temporal disconnect, however, is further evidence of the importance of paying attention to trends in disease incidence, prevalence, and YLDs and not simply focusing on mortality. Diabetes is an important example in which agestandardised prevalence rates increased $43 \%$ while death rates increased only 9\%. Stagnant or increasing age-standardised rates combined with rising mean age of the world's population implies substantial future increases in burden from these causes. The documented shift in many developed and developing countries to a larger fraction of DALYs due to YLDs is another manifestation of this global shift. Despite the evidence for this shift, global health policy discussion remains focused mostly on premature mortality. For example, in the Sustainable Development Goals Open Working Group proposal ${ }^{93}$ with 13 targets for the health goal, only one on narcotic drug abuse and harmful use of alcohol was focused on a disability.

Although the general pattern of the past 23 years has been for infectious disease mortality and morbidity, measured through incidence or prevalence, to decline, there are some very notable exceptions that stress that such trends are not inevitable with rising income per person and educational attainment. Compared with 1990, both HIV and malaria YLDs increased in 2013. More careful examination of our estimates for the six periods shows that malaria and HIV YLDs have been declining since at least 2005. Increases for dengue (nearly $450 \%$ ) and for cutaneous and mucocutaneous leishmaniasis have continued throughout the period. Increases in dengue have been ascribed to the rise of breeding sites for the mosquito vector in urban and periurban areas. ${ }^{94}$ Increases for cutaneous leishmaniasis have been related to the expansion of previously non-endemic areas as a result of urbanisation and deforestation with domestic animals as potential reservoirs. Additionally, economic hardship, natural disasters, armed conflict, movement of seasonal workers, development of new projects, and bringing non-immune labour forces into endemic areas are contributing 
causes. Also, pressure on populations throughout the world is pushing migration into areas where the infection is endemic, thereby bringing many more humans into contact with the natural vectors and resulting in increased infection rates. ${ }^{95-98}$ These counter trends for infectious diseases are a reminder that active surveillance of infectious diseases at a finegrained geospatial level are essential to detect changes that might run against the general trend towards lower rates.

The GBD 2010 reported that the burden of musculoskeletal disorders was much larger than previously appreciated. . $^{7,8,19,20,37,38}$ In this analysis, we show that musculoskeletal disorders ranged from $9.6 \%$ of YLDs to $28.9 \%$ of YLDs between 188 countries. Low back pain was the leading cause of YLDs in 86 countries and the second or third leading cause in 67 countries. Although the GBD 2010 analysis brought more attention to these disorders, there remains little policy discussion of the options available to address and prevent these disorders. ${ }^{1,99}$ From a health service point of view, it is important to note that there is a connection between the injury analysis of fractures and soft tissue injury and musculoskeletal disorders. We estimated 22 million YLDs from fractures, most of which were long-term disabilities. Most of the individuals afflicted would present with a musculoskeletal chief complaint in surveys and therefore to avoid double counting we subtracted the long-term disability from fractures and dislocations out of the estimates of the category of other musculoskeletal disorders. Musculoskeletal disorders combined with fractures and soft tissue injuries reached a total of 20.8\% of global YLDs in 2013; across countries this total ranged from $10.8 \%$ in Mali to $30.0 \%$ in South Korea. Our analysis of time trends showed that this category of disorders was an important driver of rising YLD rates per person. Increases were driven by ageing of the population in most countries with trends in obesity and physical inactivity likely exacerbating the problem. ${ }^{100,101}$ Musculoskeletal disorders were not only an important contributor to the burden of disease but were also a crucial component of health expenditure in many high-income and middleincome countries. ${ }^{102-106}$

Mental and substance abuse disorders accounted for 21.2\% of YLDs, ranging from $15.4 \%$ in Germany to $36.7 \%$ in Qatar. Major depressive disorder was a crucial contributor in developed and developing countries alike: it is the leading cause of YLDs in 56 countries, the second leading cause in 56 countries, and the third in 34 countries. Although major depressive disorder, anxiety disorders, schizophrenia, and bipolar disorder are leading causes in nearly all countries, there is much greater country variation in substance use disorders including alcohol. YLDs from alcohol use disorders ranged from a high of 2.9\% in Russia to a low of $0.3 \%$ in Iran; YLDs from drug use disorders ranged from a high of $13.5 \%$ in Qatar to a low of 0.6\% in Slovenia. New data in the GBD 2013 suggested that Iran and Afghanistan had particular problems with opioid dependence. The GBD 2013 analysis confirmed that common mental disorders had well established sex patterns in most countries: higher rates in women for major depressive disorder and anxiety and similar rates for bipolar and schizophrenia for men and women. Treatments if widely and appropriately deployed could lead to substantial reductions in the burden of these disorders; some studies suggest that up to half of YLDs could be averted in some countries. 1,107,108 As new studies accumulate on the role of childhood sexual abuse, intimate partner violence, non-sexual child abuse, and bullying, the possibility of prevention programmes is increasing. ${ }^{109-113}$ 
Systematic quantification of these risks will help establish where prevention programmes could be developed and tested.

For the first time in the GBD studies, we provide a systematic quantification of the geographical and temporal coverage of the input epidemiological data for nearly all sequelae. For several diseases with sequelae representing different levels of severity such as major depressive disorder (mild, moderate, and severe sequelae), the data representativeness metrics show there are often more data for overall disease prevalence than for determining the distribution of severity for a cause. Sequelae with the highest DRI tend to be those where administrative data such as notifiable disease reporting or hospital discharge data can be corrected for completeness or selection bias, or both. For some sequelae, such as drug use disorders only one study was available to ascertain the distribution of cases by level of severity. Despite attempts to quantify uncertainty from various sources in the estimates (see limitations below), it is highly likely that for sequelae with low DRIs uncertainty might be larger than estimated. At the global level, 12 of the top causes of YLDs had a DRI of less than $0 \cdot 25$. Priorities for further data collection in a country might usefully be informed by comparison of the estimated YLDs and the DRI for the country for each cause. Examining countries, the fraction of sequelae with any data available in a country, shows marked variation across countries within a region or within income group-eg, Nigeria with a DRI of $47 \%$ and Mauritania at $10 \%$ or the UK at $81 \%$ and Greece at $52 \%$. Our computation of the DRI was based on the data available to the GBD through published studies or reports, publicly released datasets, and unpublished data. In view of our experience in China and Mexico with subnational burden of disease estimation, it is likely data coverage could be improved through detailed national burden of disease studies, which often lead to several unpublished sources. Ministries of health and national statistics authorities in countries with low coverage should carefully assess which high burden disorders with low data availability would benefit from new data collection efforts.

With each iteration of the GBD, the cause and sequelae list has expanded. Expansion involves taking an existing cause and splitting it into more detailed component causes-eg, eating disorders were broken down into anorexia nervosa and bulimia. Residual categories, such as other mental disorders, other neurological disorders or other musculoskeletal disorders contain many rarer causes and some common causes that have very low levels of disability. One of the key values of the GBD was the comprehensive and systematic nature of the analysis; it is likely that in future iterations based on health service use data or new epidemiological studies or policy demand, the cause and sequelae list will be expanded further. The GBD cause list, even in the current expanded form, still has only 301 causes compared with the 11299 four-digit ICD-10 codes. The GBD cause list provided a more manageable public health and health service planning focused approach to the complexity of disease coding. The sequelae list also had many potential applications, such as facilitating the mapping of data collected through different studies and typologies into a common framework.

For GBD 2013, the study benefited from use of a larger computational cluster than for GBD 2010 (22 teraflops compared with 8 teraflops). The optimised recoding of DisMod-MR 1.0 to DisMod-MR 2.0, combined with greater computational capacity, allowed us to generate 
country-specific posterior estimates for incidence, prevalence, remission, and excess mortality for each of the six periods. We estimated and reported internal validity of different models and conducted cross-validation studies for ten of the models. We believe DisModMR 2.0 is a substantial improvement over DisMod-MR 1.0, but there is still much opportunity to improve these data synthesis programs in the future. In particular, work is underway to develop the next iteration of DisMod-MR that would allow for variation of incidence, remission, excess mortality rates simultaneously over age, geography, and time unlike the present approach, in which variation in rates over time is captured by independent estimation of the available data for six different periods. Despite progress in the estimation software, a fundamental challenge in epidemiological synthesis of sparse and heterogeneous data is distinguishing between true variation in the underlying rates and study heterogeneity due to variation in, for example, case definitions, assays, instruments, and sampling frames. DisMod-MR 2.0 allows explicit modelling of these factors, but true resolution of this challenge requires more data to be collected with consistent definitions and study design.

The comorbidity microsimulation for each country-age-sex-year has been used to both quantify comorbidity and allocate to contributing causes of each disability weight. By weighting each country-age-sex-year microsimulation by the true population in each age group, we have created a working model of individual health for all $7 \cdot 3$ billion individuals in the world consistent with the entire GBD non-fatal health outcome results. With future iterations of the GBD, we intend to incorporate data for risk factors into the global microsimulation providing an even more comprehensive working model of each country's population. This global and national microsimulation has many potential applications from inequality measurement to comprehensive forecasting of multiple interventions. For example, the GBD microsimulations provide an ideal environment for modelling the effect of interventions that would represent the complex interplay between disorders that is often missing in many intervention impact assessments. The distribution of functional health status across individuals in each country for different timepoints can also be used to compute measures of individual inequality in functional health. If the correlations between socioeconomic indicators within each country-age-sex-year group with health outcomes and risk factors can be estimated, then the microsimulation environment could also be used to look at the relation between health and income inequality.

Disability weights through the inclusion of new data from national surveys in Italy, Hungary, Sweden, and the Netherlands and revisions in lay descriptions for selected health states have changed from those used in the GBD 2010. The severe hearing loss disability weight increased from 0.05 to 0.18 with similar increases for profound hearing loss and deafness. Through the inclusion of incontinence in the lay descriptions for spinal cord lesion below the neck, the disability weight increased from 0.047 to 0.296 . These findings draw attention to the importance of the exact wording of lay descriptions for use in population surveys. We revised lay descriptions in the latest round of data collection based on commentary in the literature and a careful review of all lay descriptions checking for symmetry and consistency. We believe that future iterations of the GBD will benefit from further data collection and vigorous scrutiny and debate by the scientific community of the exact lay descriptions in use-the full listing is provided in the appendix pp 688-93 and further details are provided in Salomon and colleagues. ${ }^{85}$ In many countries undertaking 
subnational burden of disease studies, there may be opportunities to collect further disability weight data using the latest iteration of the GBD lay descriptions and measurement protocol. Such data would help establish whether there was any notable national variation in disability weights from the global average and strengthen the global empirical database for disability weight measurement.

Another important component determining average disability weight was the empirical analysis of the distribution of severity controlling for comorbidity. The number of studies or data sources that allowed for teasing apart functional health limitations from a particular cause from other comorbid causes were few. Very low DRIs for many sequelae in table 1 document this limitation. More datasets like the US MEPS that include both functional health limitation measurement and ICD-coded diagnoses would be extremely helpful in strengthening the empirical assessment of severity controlling for comorbidity. This must be regarded as one of the most important data gaps for the quantification of many chronic disorders.

Because the GBD 2013 has re-estimated prevalence and YLDs for all disease and injury sequelae for 1990, 1995, 2000, 2005, 2010, and 2013, we can compare the results of GBD 2013 directly with GBD 2010. The leading global causes of YLDs estimated for 2010 in this study (low back pain, major depressive disorder, iron-deficiency anaemia, neck pain, and age-related and other hearing loss) were similar to those reported in GBD 2010 (low back pain, major depressive disorder, iron-deficiency anaemia, neck pain, and COPD) for 2010. For 97 diseases or injuries, global YLDs from GBD 2013 for 2010 were significantly different from the GBD 2010 estimates for 2010. For 27 diseases and injuries, there were changes in YLDs greater than $30 \%$ but these changes were not significantly different from GBD 2010. Generally, changes stemmed from the inclusion of new data, exclusion of studies used in GBD 2010 due to changes of inclusion criteria for each sequela that emerged through the process of replicating many GBD 2010 systematic reviews, the shift to DisModMR 2.0, which reduced the effect of large sample size outlier studies, changes in covariates, changes in disability weights, or changes in severity distributions. The appendix pp 33-54 provides a more detailed account for each of these cases including changes in inclusion criteria where relevant. At the level of global YLDs, the most notable effect of these changes was the inclusion of age-related and other hearing loss in the top ten causes of global YLDs; this change was almost exclusively due to the revision of the hearing loss disability weights. The yearly updating cycle for GBD provides a much more rapid cycle for incorporating new data and scientific feedback from GBD collaborators and the broader scientific community. We expect that with each iteration there will be important changes driven by the collection and release of new data, but that the number of changes driven by uncovering older studies, exclusion of older studies on quality grounds, or changes in modelling strategy will tend to get smaller with each iteration.

A study of this scope has several limitations, many of which were detailed in the GBD 2010 analysis. ${ }^{1}$ Here we focus on selected major limitations that are not specific to the data sources or analysis of a specific sequela. First, for comorbidity simulation to work effectively, especially with the assumption of independence, individuals with a disease need to be uniquely mapped to a single sequela. For some diseases that have several distinct 
functional impairments such as motor and cognitive dysfunction for some neonatal disorders, several combinations are possible. In some cases, to map all of the possible combinations was not feasible because of increased computational load and the lack of epidemiological data to accurately describe these combinations. In a very small number of cases, we did not follow the principle of mapping one individual to a unique sequela including schistosomiasis, lymphatic filariasis, and onchocerciasis.

Second, a major limitation that might affect many sequelae is that important unpublished data sources might be missing. The collaborations with investigators in Mexico, China, and England to generate subnational burden of disease estimates for 2013 identified unpublished data sources or more detailed age and time breakdowns for published studies. Other countries are likely to have similar data sources, which after being identified could enrich the estimation of the burden of disease for a country.

Third, in this analysis of prevalence and YLDs, we attempted to capture uncertainty from model estimation and available data but $95 \%$ uncertainty intervals might be too narrow because we cannot capture uncertainty that comes from the possibility that countries with data might in some way be different for a given sequela from countries with data. Further, unlike the GBD analysis of causes of death, we do not capture uncertainty that comes from model specification. We undertook cross-validation studies for a few models but the move to using out of sample validity testing to then create ensembles of good models will require further advances in computational speed of DisMod-MR to make this viable. ${ }^{76}$

Fourth, for GBD 2013 we made a substantial effort to enhance the transparency in all aspects of the estimation. In GBD 2010, in some cases contributors who undertook systematic reviews provided results but without study references. For GBD 2013, we have included in the Global Health Data Exchange 31950 citations with metadata covering essentially all sources used in any aspect of this analysis. We have provided more detail about modelling strategies and internal validity of model fits. In view of the complexity of the GBD analysis spanning so many sequelae, estimation requires a tremendous investment across the network of collaborators measured in person-years. Replication of the entire effort was possible but would have required many resources and negotiated access to the few datasets provided to the GBD through data use agreements that are not in the public domain. With the public release of the Epi Viz tool, everyone will be able to examine the specific studies and datapoints used for each disease sequela and the model fit. To further satisfy the reasonable scientific curiosity of academics about particular sequelae, the GBD collaborators remain committed to answering detailed questions about all steps of the analysis and many disease-specific publications will follow in which much greater detail of the modelling assumptions and data for each sequela can be provided.

Fifth, when data for a sequela are collected using different definitions, assays, or instrument items, we have cross-walked between these differences using fixed effects in the metaregressions. For example, in the DisMod-MR 2.0 model for major depressive disorder, the coefficient for symptom scale measures as opposed to a diagnostic interview schedule was 0.83 (95\% uncertainty interval $0.64-1.04)$ in log space and $2.30(1.90-2.83)$ after exponentiation. By dividing symptom scale prevalence datapoints by $2 \cdot 3$, we predicted the 
corresponding values for the reference case. Another example of an important cross-walk was between data for opioid dependence from household surveys and the more inclusive estimates from triangulation of data from treatment centres, needle exchange programmes, and the justice system. ${ }^{9}$ DisMod-MR 2.0 estimated a coefficient of $0.31(0 \cdot 15-0 \cdot 74)$ after exponentiation indicating the household survey prevalence was an underestimate by more than a factor of three. Several factors could account for this large difference including lack of a steady residence, lower response to surveys, and fear of reprisals for admitting to illegal behaviours. ${ }^{114}$ We used a similar covariate for triangulated data for cocaine and amphetamine dependence but did not note systematic bias compared with household survey data. There is no equivalent approach to address the potential bias from non-response to surveys or the effect of stigma on responses for cannabis and alcohol dependence. In the DisMod-MR 2.0 model of alcohol dependence we use several study characteristics such as the type of survey and the measurement questionnaire. A review of Chinese studies on alcohol dependence showed that variation in prevalence between studies was strongly related to such study characteristics. ${ }^{115}$ Of particular interest in the Chinese review was the finding that large surveys often rely on proxy reporting for absent household members to improve the response rate and that these surveys tend to underestimate the prevalence of alcohol dependence. Although there is substantial evidence for stigma and alcohol dependence, there was no quantification of the magnitude and direction of the bias on population prevalence estimates, probably because a gold standard measurement that would be unaffected by stigma is difficult to define. ${ }^{116-118}$ Experience in the GBD 2013 compared with GBD 2010 shows that these fixed-effect coefficients for study level characteristics can be substantially affected by the inclusion of new data sources. For large cross-walks, new data in one country can alter results in many other countries. This sensitivity of statistical cross-walks in models with sparse data draws attention to the value of standardising case definitions, assays, and instruments in future data collection. The comprehensive nature of GBD provides an opportunity to identify what is the reference case approach to measurement with the current knowledge for each sequela. We plan to summarise our assessment of best practice for each sequela in a future publication.

Sixth, in models using DisMod-MR 2.0 or natural history models, the associations between incidence, remission, excess mortality, prevalence, and cause-specific mortality are modelled simultaneously. However, if there are no models for the age pattern and level of excess mortality across countries, simultaneous estimation does not ensure any correlation in the age-specific rates of prevalence and cause-specific mortality across countries. In this iteration of the GBD, we have devoted much more attention to this estimation challenge by incorporating into many models more direct information about the credible range of excess mortality across age, sex, and country. However, country variation in excess mortality has not been as extensively debated in the literature about burden of disease estimation as it has for incidence, prevalence, or causes of death. More attention to these associations and the determinants of excess mortality such as access to care will be beneficial in future iterations of the GBD.

Seventh, comorbidity simulation, used for quantifying the burden of disease and undertaking intervention analysis, has a major limitation, namely that we assume that within a countryage-sex-year group prevalence values are independent. For some disorders such as diabetes 
and ischaemic heart disease or anxiety disorders, depression, and alcohol dependence, we expect probabilities are dependent. Empirical assessments ${ }^{119,120}$ of comorbidity tend to show that age is the dominant driver of comorbidity, ignoring dependent comorbidity, for example in the MEPS data, leads to minimal errors in estimated burden. ${ }^{1}$ Incorporating dependent comorbidity into the microsimulation environment is not technically challenging; the issue is that there are insufficient data to generally estimate the age-specific correlation matrix of all 2337 sequelae. As compelling and albeit incomplete evidence on dependence accumulates, we intend to incorporate this into the comorbidity simulation microsimulations.

Eighth, in the current version of DisMod-MR 2.0, we were unable to incorporate estimates of the subnational units for China, Mexico, and the UK as a fifth level in the estimation cascade (figure 2). Instead, subnational units were modelled as though they were independent countries. If data were sparse, that meant that subnational units might borrow strength from the regional estimate rather than the country estimate. In some cases, when only national data were available, we used these data for each subnational unit after dividing the effective sample size of each datapoint by the number of subnational units to avoid overemphasising the national data used in each unit in the overall estimation. A fifth level of the cascade will be added in the next version of DisMod-MR.

Ninth, there was a steady drop in the age-standardised rates of YLDs for all injury categories between 1990 and 2013. For the unintentional injury categories, with the exception of falls, the steady drop was partly driven by a downward trend in case fatality rates accompanied by a drop in incident cases. The larger change affecting the downward trend in YLD rates was related to the difference between disability with and without treatment. In the absence of robust national data for the injury treatment rates, we have assumed access to treatment scales according to the indicator health system access. ${ }^{76}$ The health system access indicator was based on a principal component analysis of coverage of mostly maternal and child health interventions and health system infrastructure. There have been steady global improvements in health system access as captured in this indicator reducing the estimated injury YLDs.

Tenth, substantial efforts have been made in the data preparation and analysis to address issues of ascertainment bias that might affect trends. In another example of dealing with ascertainment bias we cross-walked between diagnostic assays from creatine kinasemyocardial band enzymes to troponins for the detection of acute myocardial infarction. Despite these efforts, there are some upward trends that might still relate to residual issues of ascertainment including multiple sclerosis and prostate cancer.

Eleventh, in a study with more than half a million YLD estimates generated from sometimes sparse and always disparate data sources there remain areas where additional evidence or a change in the modelling strategy could lead to better estimates. Yearly updates of GBD allowed for a continued effort to search for new data and improve methods, particularly for disorders with sparse data or inconsistencies between data sources. We are already compiling a list of issues to address in the next iteration. For example, with our collaborators in Qatar, we are searching for data to verify the high YLD estimate for opioid dependence, 
which in the models is affected by data from studies in Iran and Afghanistan. In other cases, further data for the clinical severity associated with paragonimiasis would help determine the relevance of the health state used to select the disability weight for this sequela.

Generally, we will search for additional evidence of the severity of all disorders for the next iteration of GBD.

Despite the limitations, the implications of our findings are substantial. By extending the GBD analysis to report the commonly understood measures of morbidity and disability in populations, by age, by sex, by country, and over time, this study represents an enormous resource for national, regional, and global policy debates about health priorities, not just to keep people alive well into old age, but to also keep them healthy. Without this health intelligence, large, preventable causes of health loss in populations, particularly mental and behavioural disorders and serious musculoskeletal disorders, have thus far not received the attention that they deserve in national health debates.

The GBD 2013 covers a comprehensive, exhaustive, and mutually exclusive set of causes and their sequelae at the country level over 23 years. By shifting to a process of yearly updating, the GBD provides a rapid mechanism for incorporating new data, development of methods, and new insights into old data or disease mechanisms. By steadily increasing the transparency of input sources, documenting in detail the methods used, sharing crucial code such as DisMod-MR 2.0, and facilitating online exploration of new results using dynamic data visualisations, we believe GBD can progressively become a means for global health surveillance, not just for mortality but also for reducing health loss among populations everywhere. As a group of investigators, we seek to accommodate within the GBD framework epidemiological debate about each cause, and based on scientific principles, describe the functional health of all individuals in the world. Studies such as this provide the important comprehensive, comparative, and consistent evidence to guide policy and practice, evidence that will become progressively more reliable as more data and information are identified and included as part of the global collaboration.

\section{Supplementary Material}

Refer to Web version on PubMed Central for supplementary material.

\section{Acknowledgments}

This study was funded by the Bill \& Melinda Gates Foundation. VF and RK were partly supported by the unrestricted educational grant from Lundbeck and Auckland University of Technology University. RM received funding from Ministry of Health, Labour and Welfare of Japan. LD is supported by an Australian National health and Medical Research Council (NHMRC) Principal Research Fellowship (\#1041742). Work by NY was supported by funding from the Japan Society of Clinical Pharmacology and Therapeutics. YK would like to thank the National Heart Foundation, Australia, for extending a special grant to the University of Canberra that enabled him to participate in the GBD 2013 study and more broadly engage in research on cardiovascular conditions in Australia. TW would like to acknowledge the Wellcome Trust, through whom he is supported through a Senior Research Fellowship number 091758. During the study, KJL received funding from Health Protection Scotland, the National Institute for Health Research (NIHR) Health Protection Research Unit (HPRU) in Evaluation of Interventions, and Sexual Health 24. KJL received funding from WHO to conduct the review of HSV.2 seroprevalence data that informed this study. GR's contribution to this paper has been on behalf of the International Society of Nephrology (ISN), as a follow-up of the activities of the GBD 2010 Genitourinary Diseases Expert Group. KTD would like to acknowledge the following funding source of institutional support: University of Illinois, Lemann Institute for Brazilian Studies Faculty Research Grant. KR is supported by the NIHR Oxford BRC 
and an NIHR Career Development Fellowship. FC-L would like to thank funding support by CIBERSAM, Instituto de Salud Carlos III, Spanish Ministry of Economy and Competitiveness, Madrid, Spain. LJAR would like to acknowledge the support of Qatar National Research Fund (NPRP 04-924-3-251) who provided the main funding for generating the data provided to the GBD-IHME effort. MBS is a member of the board of directors of the Anxiety and Depression Association of America (a non-profit professional and consumer organization). He has in the past 24 months been a consultant for companies that either market or are conducting research involving antidepressant or antianxiety medications: Janssen, Pfizer, and Tonix Pharmaceuticals. JAC is supported by the joint US National Institutes of Health-National Science Foundation Ecology and Evolution of Infectious Disease program (R01 TW009237) and the UK Biotechnology and Biological Sciences Research Council (BBSRC) (BB/ J010367/1), and by UK BBSRC Zoonoses in Emerging Livestock Systems awards BB/L017679, BB/L018926, and $\mathrm{BB} / \mathrm{L} 018845$. HW, AF, FC, and HE are affiliated with the Queensland Centre for Mental Health Research, which receives funding from the Queensland Department of Health. EB has received money for board membership by VIROPHARMA and EISAI; funding for travel and speaker honoraria from UCB-Pharma and GlaxoSmithKline and funding for educational presentations from GlaxoSmithKline; grants for research activities from the Italian Drug Agency, Italian Ministry of Health, Sanofi-Aventis, and the American ALS Association. ML would like to acknowledge the institutional support received from CeRIMP, Centro Regionale Infortuni e Malattie Professionali Regione Toscana (via S.Salvi, 12 - 50135 Firenze - Italy). AB would like to acknowledge funding from the Wellcome Trust. CW was supported by the NIHR Biomedical Research Centre at Guy's and St Thomas' National Health Service Foundation Trust and King's College London, funded by the NIHR. DS has received research grants or consultancy honoraria from Abbott, ABMRF, Astrazeneca, Biocodex, Eli-Lilly, GlaxoSmithKline, Jazz Pharmaceuticals, Johnson \& Johnson, Lundbeck, National Responsible Gambling Foundation, Novartis, Orion, Pfizer, Pharmacia, Roche, Servier, Solvay, Sumitomo, Sun, Takeda, Tikvah, and Wyeth. DS would like to acknowledge support by the Medical Research Council of South Africa. DAQ was supported by the Eunice Kennedy Shriver National Institute of Child Health and Human Development of the National Institutes of Health under award number 5T32HD057822. The content of this report is solely the responsibility of the authors and does not necessarily represent the official views of the National Institutes of Health. PJ is supported by a career development fellowship from the Wellcome Trust, Public Health Foundation of India and a consortium of UK universities. CK receives research grants from Brazilian public funding agencies Conselho Nacional de Desenvolvimento Cientifico e Tecnológico (CNPq), Coordenação de Aperfeiçoamento de Pessoal de Nível Superior (CAPES), and Fundacao de Amparo a Pesquisa do Estado do Rio Grande do Sul (FAPERGS). He has also received authorship royalties from publishers Artmed and Manole. RAL is partly funded through the Farr Institute at CIPHER. The Farr Institute at CIPHER is supported by a ten-funder consortium: Arthritis Research UK, the British Heart Foundation, Cancer Research UK, the Economic and Social Research Council, the Engineering and Physical Sciences Research Council, the Medical Research Council, the National Institute of Health Research, the National Institute for Social Care and Health Research (Welsh Assembly Government), the Chief Scientist Office (Scottish Government Health Directorates), the Wellcome Trust, (MRC Grant No:MR/K006525/1). JAS has received research grants from Takeda and Savient and consultant fees from Savient, Takeda, Regeneron and Allergan. JAS is a member of the executive of OMERACT, an organisation that develops outcome measures in rheumatology and receives arms-length funding from 36 companies; a member of the American College of Rheumatology's Guidelines Subcommittee of the Quality of Care Committee; and a member of the Veterans Affairs Rheumatology Field Advisory Committee. SIH is funded by a Wellcome Trust Grant (\#095066). JM is funded as a Research Career Development Fellow from the Wellcome Trust (\#089963/Z/09/Z). RL is supported by a National Health and Medical Research of Australia Fellowship. KK thanks the Director of International Institute for Population Sciences (IIPS) for giving KK the opportunity to do PhD at the IIPS, during which KK got the chance to become a GBD Study 2013 collaborator. HC is supported by the Intramural Research Program of the $\mathrm{NIH}$, the National Institute of Environmental Health Sciences. The GBD Vision Loss Expert Group received additional funding from Brien Holden Vision Institute. NP has an honorary position with the University of Melbourne, through the Centre for Eye Research Australia (CERA), and is employed by the Fred Hollows Foundation (FHF). Access to information on population-based prevalence studies from the countries they support work in was primarily as a result of her work at FHF, and the review of the manuscript and revisions suggested were a part of her position at CERA. CERA receives Operational Infrastructure CERA receives Operational Infrastructure Support funding from the Victorian Articles 56 Government. I-HO and S-JY's work was funded by a grant of the Korean Health Technology research and development project, Ministry of Health and Welfare, North Korea (grant number HI13C0729). SS is supported by grants from the NIH and employed by NRF and has honoraria from pharmaceutical companies. KD is supported by a Wellcome Trust Fellowship in Public Health and Tropical Medicine (grant number 099876). LM would like to acknowledge the Commonwealth Government of Australia and the Institute of Bone and Joint Research as funders of this work as all the original data collection for musculoskeletal was funded by these sources. BDG works for Agence de Médecine Préventive which receives grant specific support from Crucell, GlaxoSmithKline, Merck, Novartis, and SanofiPasteur. None of these sources contributed to the current work. MGS previously served as a consultant for Ellicon. DCDJ was supported by NIH grant R01 DA 003574. LJA-R would like to acknowledge the Qatar National Research Fund (NPRP 04-924-3-251) who provided the main funding for generating the data he provided to the GBD-IHME effort. The GBD Genitourinary Diseases Expert Group's activities with the GBD 2013 have been made on behalf of the International Society of Nephrology. AK would like to acknowledge funding support from Oklahoma Center for the Advancement of Science and Technology. KR is supported by the NIHR Oxford BRC and an NIHR Career Development Fellowship. KD is supported by a Wellcome Trust Training Fellowship (grant number 099876). KS 
would like to acknowledge funding from the South African Medical Research Council. IR and WHO staff acknowledge that the authors alone are responsible for the views expressed in this article and they do not necessarily represent the views, decisions or policies of the institutions with which they are affiliated.

Funding Bill \& Melinda Gates Foundation.

\section{Global Burden of Disease Study 2013 Collaborators}

Theo Vos, Ryan M Barber, Brad Bell, Amelia Bertozzi-Villa, Stan Biryukov, Ian Bolliger, Fiona Charlson, Adrian Davis, Louisa Degenhardt, Daniel Dicker, Leilei Duan, Holly Erskine, Valery L Feigin, Alize J Ferrari, Christina Fitzmaurice, Thomas Fleming, Nicholas Graetz, Caterina Guinovart, Juanita Haagsma, Gillian M Hansen, Sarah Wulf Hanson, Kyle R Heuton, Hideki Higashi, Nicholas Kassebaum, Hmwe Kyu, Evan Laurie, Xiofeng Liang, Katherine Lofgren, Rafael Lozano, Michael F MacIntyre, Maziar Moradi-Lakeh, Mohsen Naghavi, Grant Nguyen, Shaun Odell, Katrina Ortblad, David Allen Roberts, Gregory A Roth, Logan Sandar, Peter T Serina, Jeffrey D Stanaway, Caitlyn Steiner, Bernadette Thomas, Stein Emil Vollset, Harvey Whiteford, Timothy M Wolock, Pengpeng Ye, Maigeng Zhou, Marco A Ãvila*, Gunn Marit Aasvang*, Cristiana Abbafati*, Ayse Abbasoglu Ozgoren*, Foad Abd-Allah*, Muna I Abdel Aziz*, Semaw F Abera*, Victor Aboyans*, Jerry P Abraham*, Biju Abraham*, Ibrahim Abubakar*, Laith J Abu-Raddad*, Niveen ME Abu-Rmeileh*, Tania C Aburto*, Tom Achoki*, Ilana N Ackerman*, Ademola Adelekan*, Zanfina Ademi*, Arsène K Adou*, Josef C Adsuar*, Johan Arnlov*, Emilie E Agardh*, Mazin J Al Khabouri*, Sayed Saidul Alam*, Deena Alasfoor*, Mohammed I Albittar*, Miguel A Alegretti*, Alicia V Aleman*, Zewdie A Alemu*, Rafael AlfonsoCristancho*, Samia Alhabib*, Raghib Ali*, Francois Alla*, Peter Allebeck*, Peter J Allen*, Mohammad AbdulAziz AlMazroa*, Ubai Alsharif*, Elena Alvarez*, Nelson AlvisGuzman*, Omid Ameli*, Heresh Amini*, Walid Ammar*, Benjamin O Anderson*, H. Ross Anderson*, Carl Abelardo T Antonio*, Palwasha Anwari*, Henry Apfel*, Valentain S Arsic Arsenijevic *, Al Artaman*, Rana J Asghar*, Reza Assadi*, Lydia S Atkins*, Charles Atkinson*, Alaa Badawi*, Maria C Bahit*, Talal Bakfalouni*, Kalpana Balakrishnan*, Shivanthi Balalla*, Amitava Banerjee*, Suzanne L Barker-Collo, Simon Barquera*, Lars Barregard*, Lope H Barrero*, Sanjay Basu*, Arindam Basu*, Amanda Baxter*, Justin Beardsley*, Neeraj Bedi*, Ettore Beghi*, Tolesa Bekele*, Michelle L Bell*, Corina Benjet*, Derrick A Bennett*, Isabela M Bensenor*, Habib Benzian*, Eduardo Bernabe*, Tariku J Beyene*, Neeraj Bhala*, Ashish Bhalla*, Zulfiqar Bhutta*, Kelly Bienhoff*, Boris Bikbov*, Aref Bin Abdulhak*, Jed D Blore*, Fiona M Blyth*, Megan A Bohensky*, Berrak Bora Basara*, Guilherme Borges*, Natan M Bornstein*, Dipan Bose*, Soufiane Boufous*, Rupert R Bourne*, Lindsay N Boyers*, Michael Brainin*, Michael Brauer*, Carol EG Brayne*, Alexandra Brazinova*, Nicholas JK Breitborde*, Hermann Brenner*, Adam DM Briggs*, Peter M Brooks*, Jonathan Brown*, Traolach S Brugha*, Rachelle Buchbinder*, Geoffrey C Buckle*, Gene Bukhman*, Andrew G Bulloch*, Michael Burch*, Richard Burnett*, Rosario Cardenas*, Norberto L Cabral*, Ismael R Campos Nonato*, Julio C Campuzano*, Jonathan R Carapetis*, David O Carpenter*, Valeria Caso*, Carlos A Castaneda-Orjuela*, Ferran Catala-Lopez*, Vineet K Chadha*, Jung-Chen Chang*, Honglei Chen*, Wanqing Chen*, Peggy P Chiang*, Odgerel Chimed-Ochir*, Rajiv Chowdhury*, Hanne Christensen*, Costas A Christophi*, Sumeet S Chugh*, Massimo Cirillo*, Megan Coggeshall*, Aaron Cohen*, Valentina Colistro*, Samantha M Colquhoun*, Alejandra G 
Contreras*, Leslie T Cooper*, Cyrus Cooper*, Kimberly Cooperrider*, Josef Coresh*, Monica Cortinovis*, Michael H Criqui*, John A Crump*, Lucia Cuevas-Nasu*, Rakhi Dandona*, Lalit Dandona*, Emily Dansereau*, Hector G Dantes*, Paul I Dargan*, Gail Davey*, Dragos V Davitoiu*, Anand Dayama*, Vanessa De la Cruz-Gongora*, Shelley F de la Vega*, Diego De Leo*, Borja del Pozo-Cruz*, Robert P Dellavalle*, Kebede Deribe*, Sarah Derrett*, Don C Des Jarlais*, Muluken Dessalegn*, Gabrielle A deVeber*, Samath D Dharmaratne*, Cesar Diaz-Torne*, Eric L Ding*, Klara Dokova*, E R Dorsey*, Tim R Driscoll*, Herbert Duber*, Adnan M Durrani*, Karen M Edmond*, Richard G Ellenbogen*, Matthias Endres*, Sergey P Ermakov*, Babak Eshrati*, Alireza Esteghamati*, Kara Estep*, Saman Fahimi*, Farshad Farzadfar*, Derek FJ Fay*, David T Felson*, Seyed-Mohammad Fereshtehnejad*, Jefferson G Fernandes*, Cluesa P Ferri*, Abraham Flaxman*, Nataliya Foigt*, Kyle J Foreman*, F Gerry R Fowkes*, Richard C Franklin*, Thomas Furst*, Neal D Futran*, Belinda J Gabbe*, Fortune G Gankpe*, Francisco A Garcia-Guerra*, Johanna M Geleijnse*, Bradford D Gessner*, Katherine B Gibney*, Richard F Gillum*, Ibrahim A Ginawi*, Maurice Giroud*, Giorgia Giussani*, Shifalika Goenka*, Ketevan Goginashvili*, Philimon Gona*, Teresita Gonzalez de Cosio*, Richard A Gosselin*, Carolyn C Gotay*, Atsushi Goto*, Hebe N Gouda*, Richard 1 Guerrant*, Harish C Gugnani*, David Gunnell*, Rajeev Gupta*, Rahul Gupta*, Reyna A Gutierrez*, Nima Hafezi-Nejad*, Holly Hagan*, Yara Halasa*, Randah R Hamadeh*, Hannah Hamavid*, Mouhanad Hammami*, Graeme J Hankey*, Yuantao Hao*, Hilda L Harb*, Josep Maria Haro*, Rasmus Havmoeller*, Roderick J Hay*, Simon Hay*, Mohammad T Hedayati*, Ileana B Heredia Pi*, Pouria Heydarpour*, Martha Hijar*, Hans W Hoek*, Howard J Hoffman*, John C Hornberger*, H. Dean Hosgood*, Mazeda Hossain*, Peter J Hotez*, Damian G Hoy*, Mohamed Hsairi*, Howard $\mathrm{Hu}^{*}$, Guoqing Hu*, John J Huang*, Cheng Huang*, Laetitia Huiart*, Abdullatif Husseini*, Marissa Iannarone*, Kim M Iburg*, Kaire Innos*, Manami Inoue*, Kathryn H Jacobsen*, Simerjot K Jassal*, Panniyammakal Jeemon*, Paul N Jensen*, Vivekanand Jha*, Guohong Jiang*, Ying Jiang*, Jost B Jonas*, Jonathan Joseph*, Knud Juel*, Haidong Kan*, Andre Karch*, Chante Karimkhani*, Ganesan Karthikeyan*, Ronit Katz*, Anil Kaul*, Norito Kawakami*, Dhruv S Kazi*, Andrew H Kemp*, Andre P Kengne*, Yousef S Khader*, Shams Eldin AH Khalifa*, Ejaz A Khan*, Gulfaraz Khan*, Young-Ho Khang*, Irma Khonelidze*, Christian Kieling*, Daniel Kim*, Sungroul Kim*, Ruth W Kimokoti*, Yohannes Kinfu*, Jonas M Kinge*, Brett M Kissela*, Miia Kivipelto*, Luke Knibbs*, Ann Kristin Knudsen*, Yoshihiro Kokubo*, Soewarta Kosen*, Alexander Kramer*, Michael Kravchenko*, Rita V Krishnamurthi*, Sanjay Krishnaswami*, Barthelemy Kuate Defo*, Burcu Kucuk Bicer*, Ernst J Kuipers*, Veena S Kulkarni*, Kaushalendra Kumar*, G Anil Kumar*, Gene F Kwan*, Taavi Lai*, Ratilal Lalloo*, Hilton Lam*, Qing Lan*, Van C Lansingh*, Heidi Larson*, Anders Larsson*, Alicia EB Lawrynowicz*, Janet L Leasher*, Jong-Tae Lee*, James Leigh*, Ricky Leung*, Miriam Levi*, Bin Li*, Yichong Li*, Yongmei Li*, Juan liang*, Stephen Lim*, Hsien-Ho Lin*, Margaret Lind*, M Patrice Lindsay*, Steven E Lipshultz*, Shiwei Liu*, Belinda K Lloyd*, Summer Lockett Ohno*, Giancarlo Logroscino*, Katharine J Looker*, Alan D Lopez*, Nancy Lopez-Olmedo*, Joannie Lortet-Tieulent*, Paulo A Lotufo*, Nicola Low*, Robyn M Lucas*, Raimundas Lunevicius*, Ronan A Lyons*, Jixiang Ma*, Stefan Ma*, Mark T Mackay*, Marek Majdan*, Reza Malekzadeh*, Christopher C Mapoma*, Wagner Marcenes*, Lyn M March*, Chris Margono*, Guy B Marks*, Melvin B Marzan*, Joseph R Masci*, Amanda J 
Mason-Jones*, Richard G Matzopoulos*, Bongani M Mayosi*, Tasara T Mazorodze*, Neil W McGill*, John J McGrath*, Martin McKee*, Abby McLain*, Brian J McMahon*, Peter A Meaney*, Man Mohan Mehndiratta*, Fabiola Mejia-Rodriguez*, Wubegzier Mekonnen*, Yohannes A Melaku*, Michele Meltzer*, Ziad A Memish*, George Mensah*, Atte Meretoja*, Francis A Mhimbira*, Renata Micha*, Ted R Miller*, Edward J Mills*, Philip B Mitchell*, Charles N Mock*, Terrie E Moffitt*, Norlinah Mohamed Ibrahim*, Karzan A Mohammad*, Ali H Mokdad*, Glen L Mola*, Lorenzo Monasta*, Marcella Montico*, Thomas J Montine*, Ami R Moore*, Andrew E Moran*, Lidia Morawska*, Rintaro Mori*, Joanna Moschandreas*, Wilkister N Moturi*, Madeline Moyer*, Dariush Mozaffarian*, Ulrich O Mueller*, Mitsuru Mukaigawara*, Michele E Murdoch*, Joseph Murray*, Kinnari S Murthy*, Paria Naghavi*, Ziad Nahas*, Aliya Naheed*, Kovin S Naidoo*, Luigi Naldi*, Devina Nand*, Vinay Nangia*, K.M. Venkat Narayan*, Denis Nash*, Chakib Nejjari*, Sudan P Neupane*, Lori M Newman*, Charles R Newton*, Marie Ng*, Frida N Ngalesoni*, Nguyen T Nhung*, Muhammad I Nisar*, Sandra Nolte*, Ole F Norheim*, Rosana E Norman*, Bo Norrving*, Luke Nyakarahuka*, In Hwan Oh*, Takayoshi Ohkubo*, Saad B Omer*, John Nelson Opio*, Alberto Ortiz*, Jeyaraj D Pandian*, Carlo Irwin A Panelo*, Christina Papachristou*, Eun-Kee Park*, Charles D Parry*, Angel J Paternina Caicedo*, Scott B Patten*, Vinod K Paul*, Boris I Pavlin*, Neil Pearce*, Lilia S Pedraza*, Carlos A Pellegrini*, David M Pereira*, Fernando P Perez-Ruiz*, Norberto Perico*, Aslam Pervaiz*, Konrad Pesudovs*, Carrie B Peterson*, Max Petzold*, Michael R Phillips*, David Phillips*, Bryan Phillips*, Frederic B Piel*, Dietrich Plass*, Dan Poenaru*, Guilherme V Polanczyk*, Suzanne Polinder*, C A Pope*, Svetlana Popova*, Richie G Poulton*, Farshad Pourmalek*, Dorairaj Prabhakaran*, Noela M Prasad*, Dima Qato*, D A Quistberg*, Anwar Rafay*, Kazem Rahimi*, Vafa Rahimi-Movaghar*, Sajjad ur Rahman*, Murugesan Raju*, Ivo Rakovac*, Saleem M Rana*, Homie Razavi*, Amany Refaat*, Jurgen Rehm*, Giuseppe Remuzzi*, Serge Resnikoff*, Antonio L Ribeiro*, Patricia M Riccio*, Lee Richardson*, Jan Hendrik Richardus*, Anne M Riederer*, Margot Robinson*, Anna Roca*, Alina Rodriguez*, David Rojas-Rueda*, Luca Ronfani*, Dietrich Rothenbacher*, Nobhojit Roy*, George M Ruhago*, Nsanzimana Sabin*, Ralph L Sacco*, Kjetil Ksoreide*, Sukanta Saha*, Ramesh Sahathevan*, Mohammad Ali Sahraian*, Uchechukwu Sampson*, Juan R Sanabria*, Lidia Sanchez-Riera*, Itamar S Santos*, Maheswar Satpathy*, James E Saunders*, Monika Sawhney*, Mete I Saylan*, Peter Scarborough*, Ben Schoettker*, Ione JC Schneider*, David C Schwebel*, James G Scott*, Soraya Seedat*, Sadaf G Sepanlou*, Berrin Serdar*, Edson E Servan-Mori*, Katya Shackelford*, Amira Shaheen*, Saeid Shahraz*, Teresa Shamah Levy*, Siyi Shangguan*, Jun She*, Sara Sheikhbahaei*, Donald S Shepard*, Peilin Shi*, Kenji Shibuya*, Yukito Shinohara*, Rahman Shiri*, Kawkab Shishani*, Ivy Shiue*, Mark G Shrime*, Inga D Sigfusdottir*, Donald H Silberberg*, Edgar P Simard*, Shireen Sindi*, Jasvinder A Singh*, Lavanya Singh*, Vegard Skirbekk*, Karen Sliwa*, Michael Soljak*, Samir Soneji*, Sergey S Soshnikov*, Peter Speyer*, Luciano A Sposato*, Chandrashekhar T Sreeramareddy*, Heidi Stoeckl*, Vasiliki Kalliopi Stathopoulou*, Nadine Steckling*, Murray B Stein*, Dan J Stein*, Timothy J Steiner*, Andrea Stewart*, Eden Stork*, Lars J Stovner*, Konstantinos Stroumpoulis*, Lela Sturua*, Bruno F Sunguya*, Mamta Swaroop*, Bryan L Sykes*, Karen M Tabb*, Ken Takahashi*, Feng Tan*, Nikhil Tandon*, David Tanne*, Marcel Tanner*, Mohammad Tavakkoli*, Hugh R Taylor*, Braden J Te Ao*, Awoke Misganaw Temesgen*, 
Margreet Ten Have*, Eric Yeboah Tenkorang*, Abdullah Sulieman Terkawi*, Alice M Theadom*, Elissa Thomas*, Andrew L Thorne-Lyman*, Amanda G Thrift*, Imad M Tleyjeh*, Marcello Tonelli*, Fotis Topouzis*, Jeffrey A Towbin*, Hideaki Toyoshima*, Jefferson Traebert*, Bach X Tran*, Leonardo Trasande*, Matias Trillini*, Thomas Truelsen*, Ulises Trujillo*, Miltiadis Tsilimbaris*, Emin M Tuzcu*, Kingsley N Ukwaja*, Eduardo A Undurraga*, Selen B Uzun*, Wim H van Brakel*, Steven van de Vijver*, Rita Van Dingenen*, Coen H van Gool*, Yuri Y Varakin*, Tommi J Vasankari*, Monica S Vavilala*, Lennert J Veerman*, Gustavo Velasquez-Melendez*, Narayanaswamy Venketasubramanian*, Lakshmi Vijayakumar*, Salvador Villalpando*, Francesco S Violante*, Vasiliy V Vlassov*, Stephen Waller*, Mitchell T Wallin*, Xia Wan*, Linhong Wang*, JianLi Wang*, Yanping Wang*, Tati S Warouw*, Scott Weichenthal*, Elisabete Weiderpass*, Robert G Weintraub*, Andrea Werdecker*, K. Ryan R Wessells*, Ronny Westerman*, James D Wilkinson*, Hywel C Williams*, Thomas N Williams*, Solomon M Woldeyohannes*, Charles DA Wolfe*, John Q Wong*, Haidong Wong*, Anthony D Woolf*, Jonathan L Wright*, Brittany Wurtz*, Gelin Xu*, Gonghuan Yang*, Yuichiro Yano*, Muluken A Yenesew*, Gokalp K Yentur*, Paul Yip*, Naohiro Yonemoto*, SeokJun Yoon*, Mustafa Younis*, Chuanhua Yu*, Kim Yun Kim*, Maysaa El Sayed Zaki*, Yong Zhang*, Zheng Zhao*, Yong Zhao*, Jun Zhu*, David Zonies*, Joseph R Zunt*, Joshua A Salomon $\dagger$, Christopher JL Murray $\dagger$. *Authors listed alphabetically. $\dagger$ Joint senior authors.

\section{Affiliation}

Institute for Health Metrics and Evaluation (Prof T Vos PhD, R M Barber BS, B Bell PhD, A Bertozzi-Villa BA, S Biryukov BS, I Bolliger BA, D Dicker BS, C Fitzmaurice MD, T Fleming BS, N Graetz BS, J Haagsma PhD, G M Hansen MSW, S Wolf Hansen MPH, K R Heuton BChe, H Higashi PhD, H H Kyu PhD, E Laurie BS, K T Lofgren MPH, R Lozano PhD, M F MacIntyre EdM, M Moradi-Lakeh MD, M Naghavi PhD, G Nguyen BA, S Odell MD, K Ortblad MPH, D A Roberts BS, G A Roth MD, L Sandar BS, P T Serina MPH, J D Stanaway PhD, C Steiner MPH, A Stewart MPH, B Thomas MD, S E Vollset MD, T M Wolock BA, T Achoki PhD, H Apfel BA, C Atkinson BS, K Bienhoff MA, J D Blore PhD, J C Brown MAIS, M Coggeshall BA, K Cooperrider BA, L Dandona MD, E Dansereau MPH, H C Duber MD, K Estep MPA, A Flaxman PhD, K J Foreman MPH, H Hamavid BA, M L Iannarone MS, J Joseph BS, Prof S S Lim PhD, M L Lind BS, S Lockett Ohno BA, Prof A D Lopez PhD, C Margono BS, A McLain MA, A H Mokdad PhD, M L Moyer BS, P Naghavi BESc, M Ng PhD, B K Phillips BA, D E Phillips BS, L Richardson BS, M Robinson BA, K Shackelford BA, L Singh BS, P Speyer MBA, E Stork MSLIS, E Thomas MLS, H Wong PhD, B Wurtz MPH, C JL Murray DPhil), Harborview Hospital and Department of Medicine (R G Ellenbogen MD), Children's Hospital (N Kassebaum MD), Kidney Research Institute (R Katz DPhil), School of Medicine (J L Wright MD), University of Washington (R Alfonso-Cristancho MD, Prof B O Anderson MD, N D Futran MD, P N Jensen PhD, C N Mock PhD, T J Montine PhD, C A Pellegrini MD, D A Quistberg PhD, A M Riederer ScD, M S Vavilala MD, J R Zunt MD) Seattle, WA, USA; School of Population Health (D G Hoy PhD), Centre for Clinical Research (J G Scott PhD), University of Queensland, Brisbane, Australia (F C Charlson MPH, H E Erskine BPsySc, A J Ferrari 
BPsySc, H N Gouda PhD, L Knibbs PhD, Prof J J McGrath MD, R E Norman PhD, L J Veerman PhD, Prof H A Whiteford MD), Public Health England, London, UK (Prof A Davis PhD, D FJ Fay MSc); Transport and Road Safety (TARS) Research (S Boufous PhD) University of New South Wales, Sydney (Prof L Degenhardt PhD, Prof P B Mitchell MD, M Satpathy PhD), Australia; National Centers for Chronic and Noncommunible Disease Control and Prevention, Chinese Center for Disease Control, China (L Duan Master, X Liang, Y Li MD, S Liu PhD, J Ma PhD, Prof L Wang MD, P Ye PhD, Prof M Zhou PhD); National Institute for Stroke and Applied Neurosciences (V L Feigin PhD, S Balalla MPH) Auckland University of Technology (B J Te Ao MPH, A M Theadom PhD, R V Krishnamurthi PhD), Auckland, New Zealand; PATH, Seattle, WA, USA (C Guinovart $\mathrm{PhD}$ ); National Institute of Public Health, Mexico City, Mexico (M A Ãvila BS, T C Aburto MSc, S Barquera PhD, I R Campos-Nonato PhD, Prof J C Campuzano PD, A G Contreras MsC, L Cuevas-Nasu MsC, V De la Cruz-Gongora MsC, T Gonzalez de Cosio PhD, H G Dantes MsC, Prof F A Garcia-Guerra MS, I B Heredia Pi, T Shamah Levy PhD, N LopezOlmedo MSc, Prof Rafael Lozano PhD, F Mejia-Rodriguez MSc, L S Pedraza MSc, E E Servan-Mori MSc, S Villalpando PhD); Norwegian Institute of Public Health, Oslo, Norway (G Aasvang PhD, J M Kinge PhD, A K Knudsen PhD, Prof V Skirbekk PhD); La Sapienza University of Rome, Rome, Italy (C Abbafati PhD); Hacettepe University Institute of Population Studies, Turkey (A Abbasoglu Ozgoren MSc); Faculty of Medicine, Cairo University, Cairo, Egypt (Prof F Abd-Allah MD) Public Health Institute, Sudan (M I Abdel Aziz); Mekelle University, College of Health Sciences, School of Public Health, Ethiopia (S F Abera MSc, Y A Melaku MPH); Dupuytren University Hospital, Limoges, France (Prof V Aboyans MD); Oslo and Akershus University College of Applied Sciences (HiOA), Oslo, Norway (B Abraham MPhil); University of Texas School of Medicine, San Antonio, Texas, USA (J P Abraham MD); Department of Epidemiology and Public Health (H Benzian), University College (Prof I Abubakar FRCP), London, UK; Weill Cornell Medical College Qatar, Doha, Qatar (L J Abu-Raddad PhD); Institute of Community and Public HealthBirzeit University, Birzeit, Palestine (N ME Abu-Rmeileh PhD); General Practice and Primary Health Care Academic Centre (P P Chiang PhD), Centre for International Child Health (S M Colquhoun PhD), Melbourne School of Population and Global Health (Prof H R Taylor MD), University of Melbourne (I N Ackerman PhD, Z Ademi PhD, M A Bohensky PhD, Prof P M Brooks MD, Prof A D Lopez PhD, A Meretoja MD, R G Weintraub MB), Melbourne, Australia; Public Health Promotion Alliance, Osogbo, Nigeria (A Adelekan MPH); Association Ivoirienne pour le Bien-Etre Familial, Abidjan, Cote D'Ivoire (A K Adou Masters); University of Extremadura, Cáceres, Spain (Prof J C Adsuar $\mathrm{PhD}$ ); Institution of Public Health Sciences, Stockholm, Sweden (E E Agardh PhD); Ministry of Health, Muskat, Oman (M J Al Khabouri PhD, D Alasfoor MSc); International Centre for Diarrhoeal Diseases Research, Bangladesh, Dhaka, Bangladesh (S Alam MSc, A Naheed PhD); Public Health Consultant, Syria (M I Albittar MSc); Faculty of Medicine, Departamento de Medicina Preventiva y Social (M A Alegretti), School of Medicine (A V Aleman MD), Department of Medicine (V Colistro MSc), University of the Republic, Montevideo, Uruguay; Debre Markos University, Debre Markos, Amhara, Ethiopia (Z A Alemu MPH), National Guard Health Affairs, Riyadh, Saudi Arabia (S Alhabib PhD); University of Oxford, Oxford, UK (R Ali FRCP, D A Bennett PhD, A DM Briggs MRCP, S I Hay DSc, F B Piel PhD, K Rahimi DM, P Scarborough DPhil); School of Public Health, 
University of Lorraine, Nancy, France (Prof F Alla PhD); Department of Public Health Sciences (Prof P Allebeck PhD), Department of Neurobiology, Care Sciences and Society (NVS) (S Fereshtehnejad MD), Department of Medical Epidemiology and Biostatistics (Prof E Weiderpass MD), Aging Research Center (Prof M Kivipelto PhD), Karolinska Institutet (R Havmoeller PhD, S Sindi PhD), Stockholm, Sweden; Ministry of Health, Belmopan, Belize (P J Allen DDS); Saudi Ministry of Health, Riyadh, Saudi Arabia (M A AlMazroa MD, Prof Z A Memish MD); Charité - Universitätsmedizin Berlin, Berlin, Germany (U Alsharif DMD, Prof M Endres MD, S Nolte PhD, C Papachristou PhD); Government of Spain, Spain (E Alvarez PhD); Universidad de Cartagena, Cartagena de Indias, Colombia (Prof N Alvis-Guzman PhD, A J Paternina Caicedo MSc); Department of Epidemiology, College of Medicine and Health Sciences (A T Amare MPH), University Medical Center Groningen (Prof H W Hoek MD) University of Groningen, Groningen, Netherlands, Bahir Dar University (M A Yenesew), Bahir Dar, Ethiopia; Boston University, Boston, MA, USA (O Ameli MD, Prof D T Felson MD); Kurdistan Environmental Health Research Center, Kurdistan University of Medical Sciences, Sanandaj, Kurdistan, Iran (H Amini MSPH); Department of Epidemiology and Public Health, Swiss Tropical and Public Health Institute, Basel, Switzerland (H Amini MSPH, Prof M Tanner PhD); University of Basel, Basel, Switzerland (H Amini MSPH); Ministry of Public Health, Beirut, Lebanon (W Ammar PhD, H L Harb MPH); St George's (Prof H R Anderson MD), Queen Mary (Prof W Marcenes $\mathrm{PhD}$ ), University of London, London, UK; College of Public Health, University of the Philippines, Manila, Philippines (C T Antonio MD, C A Panelo MD); United Nations Population Fund, Kabul, Afghanistan (P Anwari MSc); Uppsala University, Uppsala, Norway (J Arnlov PhD, Prof A Larsson); University of Belgrade, School of Medicine, Institute of Microbiology and Immunology, Belgrade, Serbia (Prof V S Arsic Arsenijevic $\mathrm{PhD}$ ); Evidera Inc, Lexington, KY, USA (A Artaman PhD); South Asian Public Health Forum, Pakistan (R J Asghar MBBS); Mashhad University of Medical Sciences, Mashhad, Iran (R Assadi MD); Ministry Of Health, Wellness, Human Services and Gender Relations, Castries, St. Lucia (L S Atkins MPH); Public Health Agency of Canada, Toronto, ON, Canada (A Badawi PhD); INECO Neurociencias, Rosario, Santa Fe, Argentina (M C Bahit MD); Ministry of Health, Damascus, Syria (T Bakfalouni MD); Sri Ramachandra University, Chennai, Tamil Nadu, India (K Balakrishnan PhD); University of Birmingham, Birmingham, UK (A Banerjee DPhil); School of Psychology (S L Barker-Collo PhD), University of Auckland (B del Pozo-Cruz PhD), Auckland, New Zealand; Department of Occupational and Environmental Health (Prof L Barregard PhD), Health Metrics at Sahlgrenska Academy (Prof M Petzold PhD), University of Gothenburg, Gothenburg, Sweden; Department of Industrial Engineering, Pontificia Universidad Javeriana, Bogota, Colombia (L H Barrero ScD); School of Health Sciences, University of Canterbury, Christchurch, New Zealand (A Basu PhD); Stanford University, Stanford, CA, USA (S Basu $\mathrm{PhD}$ ); A Baxter; Oxford University, Ho Hi Minh City, Vietnam (J Beardsley MBChB); College of Public Health and Tropical Medicine, Jazan, Saudi Arabia (N Bedi MD); Mario Negri, Milan (E Beghi MD, Prof G Remuzzi MD), Burlo Garofolo, Trieste (L Ronfani $\mathrm{PhD}$ ), IRCCS-Istituto di Ricerche Farmacologiche, Italy; Madawalabu University, Bale Robe, Ethiopia (T Bekele MPH, M Cortinovis Biotech D, G Giussani BiolD, L Monasta DSc, M Montico MSc, N Perico MD, M Trillini MD); Yale University, New Haven, CT, USA (Prof M L Bell, J J Huang MD); National Institute of Psychiatry Ramon de la Fuente 
Muniz, Mexico City, Mexico (C Benjet PhD, Prof G Borges DSc; R A Gutierrez PhD); Hospital Universitário (I M Bensenor PhD) University of Sao Paulo (Prof A Kemp PhD, Prof P A Lotufo DrPh, Prof G V Polanczyk PhD, Prof I S Santos PhD), Sao Paulo, Brazil; King's College, London, UK (E Bernabe PhD, Prof C DA Wolfe MD); Addis Ababa University, Addis Ababa, Ethiopia (T J Beyene MSc, K Deribe MPH, W Mekonnen PhD); Wellington Hospital, Wellington, New Zealand (N Bhala DPhil); Post Graduate Institute of Medical Education and Research, Chandigarh, UT, India (A Bhalla MD); Medical Center (Z A Bhutta PhD), Aga Khan University, Karachi, Pakistan (M I Nisar MSc); I. Evdokimov Moscow State University of Medicine and Dentistry, Moscow, Russia (B Bikbov MD); University of Missouri-Kansas City, Kansas City, KS, USA (A Bin Abdulhak MD); Sydney School of Public Health (T R Driscoll PhD), Woolcock Institute of Medical Research (G B Marks PhD), University of Sydney (F M Blyth PhD, J Leigh PhD, Prof L March PhD), Sydney, Australia; Ministry of Health (S B Uzun MSc), General Directorate of Health Research, Ankara, Turkey (B Bora Basara PhD); Tel Aviv Saurasky Medical Center, Tel Aviv, Israel (Prof N M Bornstein MD); World Bank, Washington DC, DC, USA (D Bose $\mathrm{PhD}$ ); Vision \& Eye Research Unit, Anglia Ruskin University, Cambridge, UK (Prof R R Bourne FRCOphth); School of Medicine (L N Boyers BA), Neurology Department (M T Wallin MD), Georgetown University, Washington DC, DC, USA; Danube-University Krems, Krems an der Donau, Austria (Prof M Brainin MD); University of British Columbia, Vancouver, Canada (M Brauer ScD, C C Gotay PhD, F Pourmalek PhD); Cambridge Institute of Public Health, Cambridge, UK (Prof C EG Brayne MD), Trnava University, Faculty of Health Sciences and Social Work, Trnava, Slovakia (A Brazinova PhD, M Majdan PhD); University of Arizona, Tucson, AZ, USA (Prof N J K Breitborde PhD); Division of Clinical Epidemiology and Aging Research (B Schoettker PhD), German Cancer Research Center, Heidelberg, Germany (Prof H Brenner MD); University of Leicester, Leicester, UK (Prof T S Brugha MD); Monash University and Cabrini Institute, Melbourne, Australia (Prof R Buchbinder PhD); University of Massachusetts Medical School, Worcester, MA, USA (G C Buckle MPH, Prof P Gona PhD); Harvard Medical School (G Bukhman PhD), Harvard School of Public Health (E L Ding ScD, S Fahimi MD, S Shangguan MD), Harvard University (M G Shriem MD, Prof J A Salomon PhD), Cambridge, MA, USA; University of Calgary, Calgary, Alberta, Canada (A G Bulloch PhD, Prof S B Patten PhD, J Wang PhD); Great Ormond Street Hospital for Children, London, UK (M Burch MD); R Burnett; University of Joinville, Brazil (N L Cabral PhD); Telethon Institute for Child Health Research, West Perth, Australia (Prof J R Carapetis PhD); Universidad Autonoma Metropolitana, Mexico City, Mexico (R Cardenas ScD); University at Albany, Albany, NY, USA (Prof D O Carpenter MD); Stroke Unit, University of Perugia, Perugia, Italy (V Caso MD); Colombian National Health Observatory, Instituto Nacional de Salud, Bogota, Colombia (C A Castaneda-Orjuela EcoMSc); Division of Pharmacoepidemiology and Pharmacovigilance, Spanish Medicines and Healthcare Products Agency (AEMPS), Ministry of Health, Madrid, Spain (F Catala-Lopez PhD); National Tuberculosis Institute, Bangalore, India (V K Chadha MD); Institute of Epidemiology and Preventive Medicine (H-H Lin ScD), National Taiwan University (Prof JC Chang PhD), Taipei City, Taiwan, Province of China; Cancer Institute, Chinese Academy of Medical Sciences, Beijing, China (W Chen MD, PhD); Division of Intramural Research, National Institute of Environmental Health Sciences, National Institutes of Health, 
Department of Health and Human Services, RTP, NC, USA (H Chen PhD); Department of Health Development, Institute of Industrial Ecological Sciences (Y Jiang PhD, Prof K Takahashi MD), Department of Environmental Department of Environmental Epidemiology (N Chimed-Ochir MD), University of Occupational and Environmental Health, Kitakyushu City, Fukuoka Prefecture, Japan; University of Cambridge, Cambridge, UK (R Chowdhury PhD, J Murray PhD); Bispebjerg University Hospital, Copenhagen, Denmark (Prof H Christensen DMSci); Cyprus University of Technology, Limassol, Cyprus (C A Christophi $\mathrm{PhD}$; Cedars-Sinai Medical Center, Los Angeles, CA, USA (Prof S S Chugh MD); University of Salerno, Salerno, Italy (Prof M Cirillo MD; Health Effects Institute, Boston, MA, USA (A J Cohen DSc); Mayo Clinic, Rochester, MN, USA (L T Cooper MD, Prof I M Tleyjeh MD); MRC Lifecourse Epidemiology Unit, University of Southampton, Southampton, UK (Prof C Cooper FMedSci); Johns Hopkins Bloomberg School of Public Health, Baltimore, MD, USA (J Coresh PhD, B X Tran PhD) VA San Diego (S K Jassal MD), University of California (M H Criqui MD, M B Stein MD), San Diego, CA, USA; Centre for International Health, Dunedin School of Medicine (Prof J A Crump MD), University of Otago (Prof R G Poulton PhD),Dunedin, New Zealand; Indian Institute of Public Health (S Goenka PhD), Public Health Foundation of India (R Dandona PhD, G A Kumar PhD, K S Murthy MPH), Delhi, India; Guy's and St Thomas' NHS Foundation Trust, London, UK (P I Dargan FRCP); Brighton \& Sussex Medical School, Brighton, UK (G Davey MD); University of Medicine and Pharmacy Bucharest, Bucharest, Romania (D V Davitoiu PhD); Department of Surgery, Jacobi Medical Center, New York, NY, USA (A Dayama MD); Institute on Aging (S F de la Vega MD), Institute of Health Policy and Development Studies (Prof H Lam PhD), National Institutes of Health, Manila, Philippines; Griffth University, Nathan, QLD, Australia (Prof D De Leo DSc); U.S. Department of Veterans Affairs, Eastern Colorado Healthcare System, Denver, CO, USA (R P Dellavalle MD); Massey University, Palmerston North, New Zealand (S Derrett PhD); Beth Israel Medical Center, New York, NY, USA (D C Des Jarlais PhD); Africa Medical and Research Foundation in Ethiopia, Addis Ababa, Ethiopia (M Dessalegn MPH); Hospital for Sick Children, University of Toronto, Toronto, Canada (G A deVeber MD); University of Peradeniya, Peradeniya, Sri Lanka (S D Dharmaratne MD); Hospital de la Santa Creu i Sant Pau, Barcelona, Spain (C Diaz-Torne MD, PhD); Department of Social Medicine, Faculty of Public Health, Medical University of Varna, Varna, Bulgaria (K Dokova PhD); University of Rochester Medical Center, Rochester, NY, USA (Prof E R Dorsey MD); National Institute on Deafness and Other Communication Disorders (H J Hoffman MA), National Institutes of Health (A M Durrani MD), Bethesda, MD, USA; School of Medicine and Pharmacology (Prof G J Hankey MD), University of Western Australia, Crawley, WA, Australia (Prof K M Edmond PhD); The Institute of Social and Economic Studies of Population at the Russian Academy of Sciences, Chernogolovka, Russia (Prof S P Ermakov DrSc); Arak University of Medical Sciences \& Health Affairs, Arak, Markazi, Iran (B Eshrati PhD); Non-Communicable Diseases Research Center, Endocrine and Metabolic Research Institute (Prof A Esteghamati MD, F Farzadfar MD, N Hafezi-Nejad MD, S Sheikhbahaei MD), Digestive Disease Research Center (Prof R Malekzadeh MD, S G Sepanlou MD), Sina Trauma and Surgery Research Center (Prof V Rahimi-Movaghar MD), MS Research Center (M Sahraian MD), Tehran University of Medical Sciences (P Heydarpour MD), Tehran, Iran; German Hospital Oswaldo Cruz, Institute of Education and 
Sciences, Sao Paulo, Brazil (Prof J G Fernandes PhD); Federal University of Sao Paulo, Sao Paulo, Brazil (C P Ferri MD); Institute of Gerontology, Acad Med Sci, Kyiv, Ukraine (N Foigt PhD); University of Edinburgh, Edinburgh,UK (Prof F R Fowkes FRCPE); James Cook University, Townsville, QLD, Australia (R C Franklin PhD); Centre for Health Policy \& Department of Infectious Disease Epidemiology (T Furst PhD), Imperial College London, London, UK (Prof A Rodriguez PhD, M Soljak PhD); Eastern Health Clinical School (B K Lloyd PhD), Monash University, Malvern East, VIC, Australia (B J Gabbe PhD, K B Gibney FRACP, Prof A G Thrift PhD); Clinique Cooperative de Parakou, Parakou, Borgou, Benin (F G Gankpe MD); Wageningen University, Division of Human Nutrition, Wageningen, Netherlands (J M Geleijnse PhD); Agence de Medecine Preventive, Paris, France (B D Gessner MD); Howard University, Washington, DC, DC, USA (R F Gillum MD); University of Hail, College of Medicine, Hail, Saudi Arabia (I A Ginawi MD); University Hospital of Dijon, Dijon, France (Prof M Giroud MD); Ministry of Labour, Health and Social Affairs, Tbilisi, Georgia (K Goginashvili MPH); University of California in San Francisco, San Francisco, CA, USA (R A Gosselin MD, D S Kazi MD); Department of Diabetes Research, National Center for Global Health and Medicine, Tokyo, Japan (A Goto MD); University of Virginia School of Medicine (R L Guerrant MD), Department of Anesthesiology (A S Terkawi MD), University of Virginia, Charlottesville, VA, USA; Saint James School of Medicine, Netherlands Antilles (Prof H C Gugnani PhD); University of Bristol, Bristol, UK (Prof D Gunnell DSc, K J Looker PhD); Fortis Escorts Hospital, New Delhi, Delhi, India (R Gupta MD); Kanawha Charleston Health Department, Charleston, WV, USA (R Gupta PhD); School of Medicine (L Trasande MD), New York University, New York, NY, USA (Prof H Hagan PhD); Brandeis University, Waltham, MA, USA (Y Halasa MS, S Shahraz Ph, Prof D S Shepard PhD, E A Undurraga PhD); Arabian Gulf University, Manama, Bahrain (Prof R R Hamadeh DPhil); Wayne County Department of Health and Human Services, Wayne, MI, USA (M Hammami MD); School of Public Health, Sun Yae-Sen University, Guangzhou, Guangdong, China (Prof Y Hao PhD); Parc Sanitari Sant Joan de Deu, CIBERSAM, University of Barcelona, Barcelona, Spain (Prof J M Haro MD); International Foundation for Dermatology, London, UK (Prof R J Hay MD); Mazandaran University of Medical Sciences, Sari, Mazandaran, Iran (Prof M T Hedayati $\mathrm{PhD}$ ); Fundacion Entornos AC, Cuernavaca, Morelos, Mexico (M Hijar PhD); Parnassia Psychiatric Institute, The Hague, Netherlands, (Prof H W Hoek MD); Cedar Associates, Menlo Park, CA, USA (J C Hornberger MD); Albert Einstein College of Medicine, Bronx, NY, USA (Prof H Hosgood PhD); London School of Hygiene \& Tropical Medicine, London, UK (M Hossain MSc, H Larson PhD, Prof M McKee DSc, Prof N Pearce PhD, H Stoeckl PhD); Baylor College of Medicine, Waco, TX, USA (P J Hotez MD); National Institute of Public Health, Ministry of Health, Tunis, Tunisia (Prof M Hsairi MD); Public Health Division, Secretariat of the Pacific Community, Noumea, New Caledonia, Australia (D G Hoy PhD); Central South University, Changsha, China (G Hu PhD); University of Toronto, Toronto, ON, Canada (H Hu MD); George Washington University, Washington DC, USA; Centre Hospitalier Universitaire de La Réunion (L Huiart PhD); Public Health Program, Qatar University, Doha, Qatar (A Husseini PhD); Aarhus University, Aarhus, Denmark (K M Iburg PhD); National Institute for Health Development, Tallinn, Estonia (K Innos PhD); Graduate School of Medicine (M Inoue PhD), School of Public Health (Prof N Kawakami DMSc), The University of Tokyo, Tokyo, Japan; George Mason University, 
Fairfax, VA, USA (K H Jacobsen PhD); Centre for Chronic Disease Control, New Delhi, India (P Jeemon PhD, D Prabhakaran DM); Postgraduate Institute of Medical Education and Research, Chandigarh, India (Prof V Jha DM); Tianjin Centers for Diseases Control and Prevention, Tianjin, China (G Jiang MPH); Department of Ophthalmology, Medical Faculty Mannheim of the University of Heidelberg, Heidelberg, Germany (Prof J B Jonas MD); The National Institute of Public Health, Copenhagen, Denmark (Prof K Juel PhD); Zhongshan Hospital (J She PhD), Fudan University, Shanghai, China (H Kan PhD); Holtz Centre for Infection Research, Braunschweig, Germany and German Center for Infection Research (DZIF), Hannover-Braunschweig site, Hannover-Braunschweig, Germany (A Karch MD); College of Physicians and Surgeons (C Karimkhani BA), Columbia University, New York, NY, USA (A E Moran MD); All India Institute of Medical Sciences, New Delhi, India (Prof G Karthikeyan DM, Prof V K Paul MD PhD, Prof N Tandon PhD); Oklahoma State University, Stillwater, OK, USA (A Kaul MD); South African Medical Research Council, Cape Town, South Africa (A P Kengne PhD); Jordan University of Science and Technology, AlRamtha, Irbid, Jordan (Prof Y S Khader ScD); Supreme Council of Health, Doha, Qatar (AH Khalifa MSc); Health Services Academy, Islamabad, Punjab, Pakistan (E A Khan MPH); UAE University, Al Ain, Abu Dhabi, United Arab Emirates (G Khan PhD); Institute of Health Policy and Management, Seoul National University College of Medicine, Seoul, South Korea (Prof Y Khang MD); National Center for Disease Control and Public Health, Tbilisi, Georgia (I Khonelidze MP, L Sturua PhD); Federal University of Rio Grande do Sul, Rio Grande do Sul, Curitiba, Paraná, Brazil (C Kieling MD); Northeastern University, Boston, MA, USA (Prof D Kim DrPH); Soonchunhyang University, Asan, South Korea (Prof S Kim PhD); Simmons College, Boston, MA, USA (R W Kimokoti MD); University of Canberra, Canberra, ACT Australia (Y Kinfu PhD); University of Cincinnati, Cincinnati, OH, USA (B M Kissela MD); Department of Preventive Cardiology, National Cerebral and Cardiovascular Center, Suita, Japan (Y Kokubo PhD) Center for Community Empowerment, Health Policy \& Humanities (S Kosen MD), NIHRD, MoH Indonesia, Indonesia (Prof T S Warouw PhD); School of Public Health (Prof A Kramer PhD), Department of Public Health Medicine (D Plass PhD), University of Bielefeld, Bielefeld, Germany; Research Center of Neurology, Moscow, Russia (M Kravchenko PhD); Oregon Health and Science University, Portland, OR, USA (S Krishnaswami MD); Univeristy of Montreal, Montreal, Quebec, Canada (Prof B Kuate Defo PhD); Hacettepe University, Ankara, Turkey (B Kucuk Bicer $\mathrm{PhD}$ ); Department of Public Health (S Polinder PhD), University Medical Center (Prof E J Kuipers PhD, J Richardus PhD) Erasmus MC, Rotterdam, Netherlands; Arkansas State University, Jonesboro, AR, USA (V S Kulkarni PhD); International Institute for Population Sciences, Mimbai, India (K Kumar MPS); Boston Medical Center, Boston, MA, USA (G F Kwan MD); Fourth View Consulting, Tallinn, Estonia (T Lai PhD); Australian Research Centre for Population Oral Health (ARCPOH), School of Dentistry, The University of Adelaide, Gold Coast, ALD, Australia (Prof R Lalloo PhD); National Cancer Institute, Rockville, MD, USA (Q Lan PhD); IAPB and Vision 2020 LA, Weston, FL, USA (V C Lansingh PhD); Instituto Nacional de Epidemiologia "Dr. Juan H Jara", Mar del Plata, Buenos Aires, Argentina (A EB Lawrynowicz MD); Nova Southeastern University, Ft. Lauderdale, FL, USA (J L Leasher OD); Korea University, Seoul, South Korea(Prof JT Lee $\mathrm{PhD}$, S Yoon PhD); State University of New York, Albany, NY, USA (R Leung PhD); Tuscany Regional Centre for Occupational Injuries and Diseases, Florence, Italy (M Levi 
MD); Genentech, Inc., South San Francisco, CA, USA (Y Li MD); Department of Gerontology (Y Zhang PhD), Jinan Central Hospital, Jinan, Shandong, China (B Li MD); National Offce for Maternal and Child Health Surveillance, West China Second University Hospital, Sichuan University, Chengdu, Sichuan, China (Prof J Liang MD); Heart and Stroke Foundation Canada, Ottawa, ON, Canada (M P Lindsay PhD); Wayne State University, Detroit, MI, USA (S E Lipshultz MD); Turning Point, Alcohol \& Drug Centre, Eastern Health, Fitzroy, Victoria, Australia (B K Lloyd PhD); University of Bari, Bari, Italy (Prof G Logroscino PhD); American Cancer Society, Atlanta, GA, USA (J Lortet-Tieulent MSc); University of Bern, Switzerland (Prof N Low MD); The Australian National University, Canberra, ACT, Australia (Prof R M Lucas PhD); Aintree University Hospital NHS Foundation Trust, Liverpool, UK (Prof R Lunevicius PhD); Swansea University, Swansea, UK (Prof R A Lyons MD); Ministry of Health Singapore, Singapore (S Ma PhD, Z Zhao); Murdoch Children's Research Institute (R G Weintraub MB), Royal Children's Hospital, Melbourne, Australia Royal Children's Hospital Melbourne, Melbourne, Australia (M T Mackay MBBS); University of Zambia, Lusaka, Zambia (C C Mapoma PhD); University of the East Ramon Magsaysay Medical Center, Quezon City, Philippines (M B Marzan MSc); Elmhurst Hospital Center, Mount Sinai Services, Elmhurst, NY, USA (J R Masci MS); University of York, York, UK (A J Mason-Jones PhD); Medical Research Council, Cape Town, Western Cape, South Africa (R G Matzopoulos PhD, Prof C D Parry $\mathrm{PhD}$ ); Faculty of Health Sciences, Hatter Institute for Cardiovascular Research in Africa (Prof K Sliwa PhD), University of Cape Town, Cape Town, South Africa (Prof B M Mayosi DPhil, Prof D J Stein PhD); AIDC EC, Port Elizabeth, Eastern Cape, South Africa (T T Mazorodze MA); Royal Prince Alfred Hospital, Sydney, Australia (N W McGill MBBS); Alaska Native Tribal Health Consortium, Anchorage, AK, USA (B J McMahon MD); Pereleman School of Medicine (P A Meaney MD), University of Pennsylvania, Philadelphia, PA (D Silberberg MD); Janakpuri Superspecialty Hospital, New Delhi, Delhi, India (Prof M M Mehndiratta DM); Thomas Jefferson University, Philadelphia, PA, USA (M Meltzer MD); National Institutes of Health, Bethesda, MD, USA (G Mensah MD); Ifakara Health Institute, Dar es Salaam, Tanzania (F A Mhimbira MSc); Friedman School of Nutrition Science and Policy (R Micha PhD, D Mozaffarian DrPH), Tufts University, Medford, MA, USA (P Shi PhD); Pacific Institute for Research \& Evaluation, Calverton MD, USA and Centre for Population Health Research, Curtin University, Perth, Australia (T R Miller PhD); University of Ottawa, Ottawa, ON, Canada (E J Mills PhD); Duke University, Durham, NC, USA (Prof T E Mofftt PhD); Department of Medicine, Universiti Kebangsaan Malaysia Medical Center, Kuala Lampur, Malaysia (Prof N Mohamed Ibrahim MBBch); University of Salahaddin, Erbil, Iraq (K A Mohammad PhD); University of Papua New Guinea, Port Moresby, NCD, Papua New Guinea (Prof G L Mola FRCDG); University of North Texas, Denton, TX, USA (Prof A R Moore PhD); International Laboratory for Air Quality and Health, Queensland University of Technology, Brisbane, Australia (L Morawska PhD); National Center for Child Health and Development, Setagaya, Tokyo, Japan (R Mori MD); Department of Medicine (Prof M K Tsilimbaris MD), University of Crete, Crete, Greece (J Moschandreas PhD); Egerton University, Egerton, Rift Valley, Kenya (W N Moturi PhD); Philipps-University Marburg, Marburg, Germany (Prof U O Mueller PhD); Tokyo Medical and Dental University, Bunkyo-ku, Tokyo, Japan (M Mukaigawara MD); West Hertfordshire Hospitals NHS Trust, Hertfordshire, UK (M E 
Murdoch FRCP); American University of Beirut Medical Center, Beirut, Lebanon (Prof Z Nahas MD); University of KwaZulu-Natal, Durban, KwaZulu-Natal, South Africa (Prof K S Naidoo PhD); Azienda Ospedaliera papa Giovanni XXIII, Bergamo, Italy (Prof L Naldi MD); Ministry of Health Fiji, Suva, Republic of Fiji (D Nand MPH); Suraj Eye Institute, Nagpur, Maharashtra, India (Prof V Nangia FRCS); Rollins School of Public Health (E P Simard PhD), Emory University, Atlanta, GA, USA (Prof KM V Narayan MD, B Omer PhD, Prof M R Phillips MD); School of Public Health, City University of New York, New York, NY, USA (D Nash PhD); Faculty of Medicine, Fez, Morocco (Prof C Nejjari PhD); Norwegian Center for Addiction Research (SERAF), University of Oslo, Oslo, Norway (S P Neupane Mphil); WHO Regional Office for Europe in Denmark (I Rakovac PhD), World Health Organization, Geneva, Switzerland (L Newman MD); KEMRI Wellcome Trust, Kilifi, Kenya (Prof C R Newton MD); Ministry of Health and Social Welfare, Dar es Salaam,Tanzania (F N Ngalesoni MSc); Hanoi School of Public Health, Hanoi, Vietnam (N T Nhung MPH); University of Bergen, Bergen, Norway (Prof O F Norheim PhD); Department of Clinical Sciences, Medical Faculty, Lund University, Lund, Sweden (Prof B Norrving PhD); Makerere University, Kampala, Uganda (L Nyakarahuka MPH); Kyung Hee University, Seoul, Korea (I-H Oh PhD); Teikyo University School of Medicine, Itabashi, Tokyo, Japan (Prof T Ohkubo MD); Lira District Local Government, Lira Municipal Council, Northern Uganda, Uganda (J Opio MPH); IIS-Fundacion Jimenez Diaz, Madrid, Spain (Prof A Ortiz PhD); Christian Medical College Ludhiana, Ludhiana, India (J D Pandian MD); Kosin University College of Medicine, Busan, South Korea (E-K Park $\mathrm{PhD}$ ); Independent Researcher, Port Moresby, Papua New Guinea (B I Pavlin MD); REQUIMTE/Laboratario de Farmacognosia, Departamento de Quamica, Faculdade de Farma;cia, Universidade do Porto, Portugal (Prof D M Pereira PhD); Hospital Universitario Cruces and Biocruces Health Research Institute, Barakaldo, Bizkaia, Spain (F P Perez-Ruiz $\mathrm{PhD}$ ); Postgraduate Medical Institute, Lahore, Pakistan (A Pervaiz MHA); Flinders University, Bedford Park, SA, Australia (Prof K Pesudovs PhD); Aalborg University, Aalborg, Denmark (C B Peterson PhD); School of Public Health, Faculty of Health Sciences, University of the Witwatersrand, Johannesburg, South Africa (Prof M Petzold $\mathrm{PhD}$ ); Shanghai Jiao Tong University, Shanghai, China (Prof M R Phillips MD); McMaster University, Hamilton, ON, Canada (D Poenaru MD); Brigham Young University, Provo, UT, USA (Prof C A Pope III PhD); Centre for Addiction and Mental Health, Toronto, ON, Canada (S Popova MD, Prof J Rehm PhD); The Fred Hollows Foundation, Sydney, Australia (N M Prasad MD); College of Pharmacy, University of Illinois, Chicago, IL, USA (D Qato PhD); Contech International, Lahore, Pakistan (A Rafay MBBS); Hamad Medical Corporation, Doha, Qatar (S ur Rahman FRCPCH); University of Missouri, Columbia, MO, USA (M Raju PhD); Department of Public Health, University of the Punjab, Lahore, Pakistan (M Rana PhD); Center for Disease Analysis, Louisville, CO, USA (H Razavi PhD); Walden University, Minneapolis, MN, USA (Prof A Refaat PhD); International Health and Development, Switzerland (Prof S Resnikoff PhD); Hospital das Clinicas (Prof A L Ribeiro MD), Escola de Enfermagem (Prof G Velasquez-Melendez PhD), Universidade Federal de Minas Gerais, Belo Horizonte, Brazil; Department of Clinical Neurological Sciences, London Health Sciences Centre, University of Western Ontario, London, ON, Canada (P M Riccio MD); MRC Unit, Fajara, The Gambia (A Roca PhD); Centre of Research in Environmental Epidemiology (CREAL), Barcelona, Spain (D Rojas-Rueda PhD); Institute 
of Epidemiology and Medical Biometry, Ulm University, Ulm, Germany (Prof D Rothenbacher MD); BARC Hospital, Mumbai, Maharashtra, India (N Roy MD); Muhimbili University of Health and Allied Sciences, Dar es Salaam, Tanzania (G M Ruhago MA, B F Sunguya PhD); Rwanda Bio-Medical Center, Kigali, Rwanda (N Sabin MD); University of Miami, Miller School of Medicine, Miami, FL, USA (R L Sacco MD); Queensland Centre for Mental Health Research, The Park for Centre for Mental Health, Wacol, QLD, Australia (S Saha PhD); Universiti Kebangsaan Malaysia Medical Centre, Bangi, Selangor, Malaysia (R Sahathevan PhD); Vanderbilt University, Nashville, TN, USA (U Sampson MPH); Case Western Reserve University, Cleveland, OH, USA (J Sanabria MD); Mafraq HospitalSEHA, Abu Dhabi, United Arab Emirates (L Sanchez-Riera MD); Dartmouth Hitchcock Medical Center, Lebanon, NH, USA (J E Saunders MD); Marshall University, Huntington, WV, USA (M Sawhney PhD); Novartis, Istanbul, Turkey (M I Saylan MD); Federal University of Santa Catarina, Florianópolis, SC, Brazil (I JC Schneider PhD); University of Alabama at Birmingham, Birmingham, AL, USA (D C Schwebel PhD, J A Singh MD); Stellenbosch University, Stellenbosch, South Africa (Prof S Seedat PhD); University of Colorado, Boulder, CO, USA (B Serdar PhD); An-Najah University, Nablus, Palestine (A Shaheen PhD); Tokyo University, Tokyo, Japan (K Shibuya MD); Tachikawa Hospital, Tokyo, Japan (Y Shinohara MD); Finnish Institute of Occupational Health, Finland (R Shiri $\mathrm{PhD}$ ); Washington State University, Pullman, WA, USA (K Shishani PhD); Heriot-Watt University, Edinburgh, Scotland, UK (I Shiue PhD); Reykjavik University, Reykjavik, Iceland (I D Sigfusdottir PhD); Dartmouth College, Hannover, NH, USA (S Soneji PhD); Stavanger University Hospital, Stavanger, Norway (K Soreide PhD); Federal Research Institute for Health Organization and Informatics of Ministry of Health of the Russian Federation, Moscow, Russia (S S Soshnikov PhD); Department of Clinical Neurological Sciences. Western University, London, ON, Canada (L A Sposato MD); Faculty of Medicine and Health Sciences, University Tunku Abdul Rahman, Selangor, Malaysia (C T Sreeramareddy MD); Centre Hospitalier Nord Deux-Sevres, Bressuire, France (V K Stathopoulou MD); University Hospital Munich, Institute and Outpatient Clinic for Occupational, Social and Environmental Medicine, Munich, Germany (N Steckling MSc); Department of Neuroscience (Prof L J Stovner PhD), Norwegian University of Science and Technology, Trondheim, Norway (Prof T J Steiner PhD); KEELPNO, Centre for Disease Control, Greece, dispatched to "Alexandra" General Hospital of Athens, Athens, Greece (K Stroumpoulis PhD); Northwestern University, Evanston, IL, USA (M Swaroop MD); Department of Criminology, Law and Society and Sociology, University of CaliforniaIrvine, Irvine, CA, USA (B L Sykes PhD); University of Illinois, Urbana, IL, USA (K M Tabb PhD); National Institute of Occupational Health and Poison Control, China CDC, China (Prof F Tan MD); Chaim Sheba Medical Center and Tel Aviv University, Tel Aviv, Israel (Prof D Tanne MD); Westchester Medical Center, Valhalla, NY, USA (M Tavakkoli MD); Jhpiego, an affilate of Johns Hopkins University, Addis Ababa, Ethiopia (A M Temesgen PhD); Netherlands Institute of Mental Health and Addiction, Utrecht, Netherlands (M Ten Have PhD); Memorial University, St John's, Newfoundland, Canada (E Y Tenkorang PhD); Department of Anesthesiology (A S Terkawi MD), College of Medicine (Prof I M Tleyjeh MD), King Fahad Medical City, Riyadh, Saudi Arabial WorldFish, Penang, Malaysia (A L Thorne-Lyman ScD); University of Alberta, Edmonton, AL, Canada (M Tonelli MD; Aristotle University of Thessaloniki, Thessaloniki, Greece (Prof F Topouzis 
$\mathrm{PhD}$ ); Cincinnati Children's Hospital Medical Center, Cincinnati, OH, USA (Prof J A Towbin MD); Health Care Center of Anjo Kosei Hospital, Anjo City, Aichi Prefecture, Japan (H Toyoshima MD); University of Southern Santa Catarina, Palhoça, Santa Catarina, Brazil (Prof J Traebert PhD); Department of Neurology, Copenhagen University Hospital Herlev, Herlev, Copenhagen, Denmark (T Truelsen DMSc); Servicio Canario de Salud, Santa Cruz de Tenerife, Tenerife, Spain (U Trujillo MD); Cleveland Clinic, Cleveland, OH, USA (Prof E M Tuzcu MD); Department of Internal Medicine, Federal Teaching Hospital Abakaliki, Abakailiki, Ebonyi State, Nigeria (K N Ukwaja MBBS); Netherlands Leprosy Relief, Netherlands (W H van Brakel PhD); African Population and Health Research Center, Nairobi, Kenya (S van de Vijver MD); European Commission, Joint Research Centre, Ispra, Italy (R Van Dingenen PhD); National Institute for Public Health and the Environment, Bilthoven, Netherlands (C H van Gool PhD); Research Center of Neurology, Moscow, Russia (Prof Y Y Varakin MD); UKK Institute for Health Promotion Research, Tampere, Finland (Prof T J Vasankari PhD); Neuroscience Centre, Raffles Hospital, Singapore (N Venketasubramanian FRCP); Voluntary Health Services, Sneha, India (Prof L Vijayakumar $\mathrm{PhD}$ ); University of Bologna, Bologna, Italy (Prof F S Violante MD); Higher School of Economics, Moscow, Russia (Prof V V Vlassov MD); Uniformed Services University of Health Sciences, Bethesda, MD, USA (S Waller MD); VA Medical Center, Washington, DC, USA (M T Wallin MD); Institute of Basic Medical Sciences, Chinese Academy of Medical Sciences, Beijing, China (X Wan PhD); National Office for Maternal and Child's Health Surveillance, China (Prof Y Wang MD); Health Canada, Ottawa, ON, Canada (S Weichenthal PhD); Institute of Medical Sociology and Social Medicine, Germany (A Werdecker Dipl.oec.troph); University of California at Davis, Davis, CA, USA (K R Wessells PhD); University of Marburg, Hessen, Germany (R Westerman PhD); University of Miami, Miami, FL, USA (Prof J D Wilkinson MD); Imperial College, London, UK (Prof T N Williams MBBS); University of Nottingham, Nottingham, UK (Prof H C Williams DSc); Institute of Public Health, University of Gondar, Amhara, Ethiopia (S M Woldeyohannes MPH); Ateneo School of Medicine and Public Health, Pasig City, Manila, Philippines (J Q Wong MD); Royal Cornwall Hospital, Truro, Cornwall, UK (Prof A D Woolf FRCP); Nanjing University School of Medicine, Jinling Hospital, Nanjing, China (Prof G Xu MD); G Yang MD; Division of Cardiovascular Medicine, Jichi Medical University School of Medicine, Shimotsuke, Tochigi, Japan (Y Yano MD); General Directorate of Health Research, Ankara, Turkey (G K Yentur PhD); The University of Hong Kong, Hong Kong, China (Prof P Yip PhD); National Center of Neurology and Psychiatry, Kodaira, Tokyo, Japan (N Yonemoto MPH); Jackson State University, Jackson, MS, USA (Prof M Younis PhD); Department of Epidemiology and Biostatistics, School of Public Health, Wuhan University and Global Health Institute, Wuhan University, Wuhan, Hubei, China (Prof C Yu PhD); TCM Medical TK SDN BHD TCM, Nusajaya, Johor Bahru, Malaysia (K Yun Kin PhD); Mansoura School of Medicine, Mansoura, Egypt (Prof M ES Zaki MD); Chongqing Medical University, China (Prof Y Zhao Masters); National Office of MCH Surveillance of China, China (Prof J Zhu MD); Landstuhl Regional Medical Center, USA (D Zonies MD) 


\section{References}

1. Vos T, Flaxman AD, Naghavi M, et al. Years lived with disability (YLDs) for 1160 sequelae of 289 diseases and injuries 1990-2010: a systematic analysis for the Global Burden of Disease Study 2010. Lancet. 2012; 380:2163-96. [PubMed: 23245607]

2. Baxter AJ, Brugha TS, Erskine HE, Scheurer RW, Vos T, Scott JG. The epidemiology and global burden of autism spectrum disorders. Psychol Med. 2014; 45:1-13. [PubMed: 25065902]

3. Blencowe H, Vos T, Lee ACC, et al. Estimates of neonatal morbidities and disabilities at regional and global levels for 2010: introduction, methods overview, and relevant findings from the Global Burden of Disease study. Pediatr Res. 2013; 74(suppl 1):4-16. [PubMed: 24366460]

4. Boyers LN, Karimkhani C, Hilton J, Richheimer W, Dellavalle RP. Global Burden of Eye and Vision Disease as Reflected in the Cochrane Database of Systematic Reviews. JAMA Ophthalmol. 2014 published online Sept 18. DOI:10.1001/jamaophthalmol.2014.3527.

5. Charlson FJ, Ferrari AJ, Flaxman AD, Whiteford HA. The epidemiological modelling of dysthymia: application for the Global Burden of Disease Study 2010. J Affect Disord. 2013; 151:111-20. [PubMed: 23806588]

6. Chugh SS, Havmoeller R, Narayanan K, et al. Worldwide epidemiology of atrial fibrillation: a Global Burden of Disease 2010 Study. Circulation. 2014; 129:837-47. [PubMed: 24345399]

7. Cross M, Smith E, Hoy D, et al. The global burden of rheumatoid arthritis: estimates from the global burden of disease 2010 study. Ann Rheum Dis. 2014; 73:1316-22. [PubMed: 24550173]

8. Cross M, Smith E, Hoy D, et al. The global burden of hip and knee osteoarthritis: estimates from the global burden of disease 2010 study. Ann Rheum Dis. 2014; 73:1323-30. [PubMed: 24553908]

9. Degenhardt L, Charlson F, Mathers B, et al. The global epidemiology and burden of opioid dependence: results from the global burden of disease 2010 study. Addiction. 2014; 109:1320-33. [PubMed: 24661272]

10. Degenhardt L, Ferrari AJ, Calabria B, et al. The global epidemiology and contribution of cannabis use and dependence to the global burden of disease: results from the GBD 2010 study. PLoS One. 2013; 8:e76635. [PubMed: 24204649]

11. Degenhardt L, Whiteford HA, Ferrari AJ, et al. Global burden of disease attributable to illicit drug use and dependence: findings from the Global Burden of Disease Study 2010. Lancet. 2013; 382:1564-74. [PubMed: 23993281]

12. Driscoll T, Jacklyn G, Orchard J, et al. The global burden of occupationally related low back pain: estimates from the Global Burden of Disease 2010 study. Ann Rheum Dis. 2014; 73:975-81. [PubMed: 24665117]

13. Erskine HE, Ferrari AJ, Nelson P, et al. Epidemiological modelling of attention-deficit/ hyperactivity disorder and conduct disorder for the Global Burden of Disease Study 2010. J Child Psychol Psychiatry. 2013; 54:1263-74. [PubMed: 24117530]

14. Erskine HE, Ferrari AJ, Polanczyk GV, et al. The global burden of conduct disorder and attentiondeficit/hyperactivity disorder in 2010. J Child Psychol Psychiatry. 2014; 55:328-36. [PubMed: 24447211]

15. Feigin VL, Forouzanfar MH, Krishnamurthi R, et al. Global Burden of Diseases, Injuries, and Risk Factors Study 2010 (GBD 2010); GBD Stroke Experts Group. Global and regional burden of stroke during 1990-2010: findings from the Global Burden of Disease Study 2010. Lancet. 2014; 383:245-54. [PubMed: 24449944]

16. Hay RJ, Johns NE, Williams HC, et al. The global burden of skin disease in 2010: an analysis of the prevalence and impact of skin conditions. J Invest Dermatol. 2014; 134:1527-34. [PubMed: 24166134]

17. Higashi H, Barendregt JJ, Kassebaum NJ, Weiser TG, Bickler SW, Vos T. The burden of selected congenital anomalies amenable to surgery in low and middle-income regions: cleft lip and palate, congenital heart anomalies and neural tube defects. Arch Dis Child. 2014 published online Sept 26. DOI:10.1136/archdischild-2014-306175.

18. Hotez PJ, Alvarado M, Basáñez M-G, et al. The global burden of disease study 2010: interpretation and implications for the neglected tropical diseases. PLoS Negl Trop Dis. 2014; 8:e2865.

[PubMed: 25058013] 
19. Hoy D, March L, Brooks P, et al. The global burden of low back pain: estimates from the Global Burden of Disease 2010 study. Ann Rheum Dis. 2014; 73:968-74. [PubMed: 24665116]

20. Hoy D, March L, Woolf A, et al. The global burden of neck pain: estimates from the global burden of disease 2010 study. Ann Rheum Dis. 2014; 73:1309-15. [PubMed: 24482302]

21. Hoy DG, Smith E, Cross M, et al. The global burden of musculoskeletal conditions for 2010: an overview of methods. Ann Rheum Dis. 2014; 73:982-89. [PubMed: 24550172]

22. IHME. The Global Burden of Disease: Generating Evidence, Guiding Policy - East Asia and Pacific Regional Edition. Institute for Health Metrics and Evaluation, Human Development Network, World Bank; Seattle, WA: 2013.

23. IHME. The Global Burden of Disease: Generating Evidence, Guiding Policy - Europe and Central Asia Regional Edition. Institute for Health Metrics and Evaluation, Human Development Network, World Bank; Seattle, WA: 2013.

24. IHME. The Global Burden of Disease: Generating Evidence, Guiding Policy - Latin America and Caribbean Regional Edition. Institute for Health Metrics and Evaluation, Human Development Network, World Bank; Seattle, WA: 2013.

25. IHME. The Global Burden of Disease: Generating Evidence, Guiding Policy - Middle East and North Africa Regional Edition. Institute for Health Metrics and Evaluation, Human Development Network, World Bank; Seattle, WA: 2013.

26. IHME. The Global Burden of Disease: Generating Evidence, Guiding Policy - South Asia Regional Edition. Institute for Health Metrics and Evaluation, Human Development Network, World Bank; Seattle, WA: 2013.

27. IHME. The Global Burden of Disease: Generating Evidence, Guiding Policy - Sub-Saharan Africa Regional Edition. Institute for Health Metrics and Evaluation, Human Development Network, World Bank; Seattle, WA: 2013.

28. Leonardi M, Raggi A. Burden of migraine: international perspectives. Neurol Sci. 2013; 34(suppl 1):S117-18. [PubMed: 23695058]

29. Mensah GA, Forouzanfar MH, Naghavi M, et al. Comparable estimates of mortality and trends for cardiovascular disease including congenital heart disease in 21 world regions in 1990 and 2010: the Global Burden of Diseases, Injuries and Risk Factors Study. J Am Coll Cardiol. 2013; 61:E1406.

30. Moran AE, Forouzanfar MH, Roth GA, et al. Temporal trends in ischemic heart disease mortality in 21 world regions, 1980 to 2010: the Global Burden of Disease 2010 study. Circulation. 2014; 129:1483-92. [PubMed: 24573352]

31. Moran AE, Forouzanfar MH, Roth GA, et al. The global burden of ischemic heart disease in 1990 and 2010: the Global Burden of Disease 2010 study. Circulation. 2014; 129:1493-501. [PubMed: 24573351]

32. Murray CJL, Richards MA, Newton JN, et al. UK health performance: findings of the Global Burden of Disease Study 2010. Lancet. 2013; 381:997-1020. [PubMed: 23668584]

33. Pederson H, Okland T, Boyers LN, et al. Identifying Otolaryngology Systematic Review Research Gaps: Comparing Global Burden of Disease 2010 Results With Cochrane Database of Systematic Review Content. JAMA Otolaryngol Head Neck Surg. 2015; 141:67-72. [PubMed: 25356671]

34. Powles J, Fahimi S, Micha R, et al. Global Burden of Diseases Nutrition and Chronic Diseases Expert Group (NutriCoDE). Global, regional and national sodium intakes in 1990 and 2010: a systematic analysis of $24 \mathrm{~h}$ urinary sodium excretion and dietary surveys worldwide. BMJ Open. 2013; 3:e003733.

35. Pullan RL, Smith JL, Jasrasaria R, Brooker SJ. Global numbers of infection and disease burden of soil transmitted helminth infections in 2010. Parasit Vectors. 2014; 7:37. [PubMed: 24447578]

36. Risal A, Manandhar K, Steiner TJ, Holen A, Koju R, Linde M. Estimating prevalence and burden of major disorders of the brain in Nepal: cultural, geographic, logistic and philosophical issues of methodology. J Headache Pain. 2014; 15:51. [PubMed: 25127664]

37. Smith E, Hoy D, Cross M, et al. The global burden of gout: estimates from the Global Burden of Disease 2010 study. Ann Rheum Dis. 2014; 73:1470-76. [PubMed: 24590182] 
38. Smith E, Hoy DG, Cross M, et al. The global burden of other musculoskeletal disorders: estimates from the Global Burden of Disease 2010 study. Ann Rheum Dis. 2014; 73:1462-69. [PubMed: 24590181]

39. Whiteford HA, Degenhardt L, Rehm J, et al. Global burden of disease attributable to mental and substance use disorders: findings from the Global Burden of Disease Study 2010. Lancet. 2013; 382:1575-86. [PubMed: 23993280]

40. Ferrari AJ, Charlson FJ, Norman RE, et al. Burden of depressive disorders by country, sex, age, and year: findings from the global burden of disease study 2010. PLoS Med. 2013; 10:e1001547. [PubMed: 24223526]

41. Karimkhani C, Boyers LN, Prescott L, et al. Global burden of skin disease as reflected in Cochrane Database of Systematic Reviews. JAMA Dermatol. 2014; 150:945-51. [PubMed: 24807687]

42. Krishnamurthi RV, Feigin VL, Forouzanfar MH, et al. Global Burden of Diseases, Injuries, Risk Factors Study 2010 (GBD 2010); GBD Stroke Experts Group. Global and regional burden of firstever ischaemic and haemorrhagic stroke during 1990-2010: findings from the Global Burden of Disease Study 2010. Lancet Glob Health. 2013; 1:e259-81. [PubMed: 25104492]

43. Kassebaum NJ, Jasrasaria R, Naghavi M, et al. A systematic analysis of global anemia burden from 1990 to 2010. Blood. 2014; 123:615-24. [PubMed: 24297872]

44. Kassebaum NJ, Bernabé E, Dahiya M, Bhandari B, Murray CJL, Marcenes W. Global burden of severe periodontitis in 1990-2010: a systematic review and meta-regression. J Dent Res. 2014; 93:1045-53. [PubMed: 25261053]

45. Moran A, Forouzanfar M, Sampson U, Chugh S, Feigin V, Mensah G. The epidemiology of cardiovascular diseases in sub-Saharan Africa: the Global Burden of Diseases, Injuries and Risk Factors 2010 Study. Prog Cardiovasc Dis. 2013; 56:234-39. [PubMed: 24267430]

46. Stovner LJ, Hoff JM, Svalheim S, Gilhus NE. Neurological disorders in the Global Burden of Disease 2010 study. Acta Neurol Scand Suppl. 2014; 129:1-6. [PubMed: 24588499]

47. Pasricha S-R. Anemia: a comprehensive global estimate. Blood. 2014; 123:611-12. [PubMed: 24482500]

48. Bittles AH. Genetics and global healthcare. J R Coll Physicians Edinb. 2013; 43:7-10. [PubMed: 23516683]

49. Byass P, de Courten M, Graham WJ, et al. Reflections on the global burden of disease 2010 estimates. PLoS Med. 2013; 10:e1001477. [PubMed: 23843748]

50. Gabbe BJ, Lyons RA, Harrison JE, et al. Validating and Improving Injury Burden Estimates Study: the Injury-VIBES study protocol. Inj Prev. 2013 published online Aug 6. DOI:10.1136/ injuryprev-2013-040936.

51. Spencer S. Global Burden of Disease 2010 Study: a personal reflection. Glob Cardiol Sci Pract. 2013; 2013:115-26. [PubMed: 24689009]

52. Hser Y-I, Evans E, Grella C. Commentary on Degenhardt et al. (2014): Regional variation in the global burden of disease attributable to opioid dependence-where do the data come from and does population size matter? Addiction. 2014; 109:1334-35. [PubMed: 25041203]

53. Nord E. Disability weights in the Global Burden of Disease 2010: unclear meaning and overstatement of international agreement. Health Policy. 2013; 111:99-104. [PubMed: 23608637]

54. Taylor HR, Jonas JB, Keeffe J, et al. Disability weights for vision disorders in Global Burden of Disease study. Lancet. 2013; 381:23. [PubMed: 23266227]

55. Voigt K, King NB. Disability weights in the global burden of disease 2010 study: two steps forward, one step back? Bull World Health Organ. 2014; 92:226-28. [PubMed: 24700983]

56. GBD 2010 Country Collaboration. GBD 2010 country results: a global public good. Lancet. 2013; 381:965-70. [PubMed: 23668561]

57. Gilmour S, Liao Y, Bilano V, Shibuya K. Burden of disease in Japan: using national and subnational data to inform local health policy. J Prev Med Public Health. 2014; 47:136-43. [PubMed: 24921016]

58. Public Health Policy \& Strategy Unit/NHS Commissioning Unit. Living well for longer: a call to action to reduce avoidable premature mortality. UK Department of Health; 2013. 
59. [accessed May 11, 2015] The global burden of disease and its implications for U.S. Policy. Council on Foreign Relations. http://www.cfr.org/world/global-burden-disease-its-implications-us-policy/ p35394

60. Yu SC, Tan F, Zhou MG, Liu SW, Zhu XJ, Zhu YL. Global Burden of Disease, Injury and Risk Factor Study 2010: its policy implications for China. Biomed Environ Sci. 2014; 27:45-48. [PubMed: 24553373]

61. Murray CJL, Ortblad KF, Guinovart C, et al. Global, regional, and national incidence and mortality for HIV, tuberculosis, and malaria during 1990-2013: a systematic analysis for the Global Burden of Disease Study 2013. Lancet. 2014; 384:1005-70. [PubMed: 25059949]

62. Flaxman, A.; Murray, C.; Vos, T., editors. Integrated meta-regression framework for descriptive epidemiology. University of Washington Press; Seattle, WA: 2014.

63. Bhatt S, Gething PW, Brady OJ, et al. The global distribution and burden of dengue. Nature. 2013; 496:504-07. [PubMed: 23563266]

64. London School of Hygiene and Tropical Medicine. GAHI: Global Atlas of Helminth Infections; 2014. published online Oct 25. http://www.thiswormyworld.org/ [accessed Oct 25, 2014]

65. Forouzanfar MH, Foreman KJ, Delossantos AM, et al. Breast and cervical cancer in 187 countries between 1980 and 2010: a systematic analysis. Lancet. 2011; 378:1461-84. [PubMed: 21924486]

66. Lozano R, Naghavi M, Foreman K, et al. Global and regional mortality from 235 causes of death for 20 age groups in 1990 and 2010: a systematic analysis for the Global Burden of Disease Study 2010. Lancet. 2012; 380:2095-128. [PubMed: 23245604]

67. National Cancer Institute (United States). United States - SEER Cancer Statistics Review (CSR) 1975-2011. National Cancer Institute; Bethesda, MD: 2014. http://ghdx.healthdata.org/record/ united-states-seer-cancer-statistics-review-csr-1975-2011 [accessed Nov 19, 2014]

68. Sankaranarayanan, R.; Swaminathan, R.; Lucas, E. Cancer survival in Africa, Asia, the Caribbean and Central America (SurvCan). International Agency for Research on Cancer; Lyon, France: 2011. IARC Scientific Publications, number 162

69. Agency for Healthcare Research and Quality. Agency for Healthcare Research and Quality. United States Medical Expenditure Panel Survey 1996-2012. Agency for Healthcare Research and Quality; Rockville, MD:

70. Centers for Disease Control and Prevention (CDC). Medical University of South Carolina. South Carolina Department of Disabilities and Special Needs. South Carolina Department of Health and Environmental Control. United States - South Carolina Traumatic Brain Injury Follow-up Registry 1999-2013. USA:

71. Haagsma JA. Posttraumatic Stress Disorder Following Injury: Trajectories and Impact on HealthRelated Quality of Life. J Depress Anxiety. 2013; 14:1242-49.

72. Johns Hopkins Bloomberg School of Public Health; University of Washington, Westat. United States national study on the costs and outcomes of trauma care 2001-2003.

73. Polinder S, van Beeck EF, Essink-Bot ML, et al. Functional outcome at 2.5, 5, 9, and 24 months after injury in the Netherlands. J Trauma. 2007; 62:133-41. [PubMed: 17215744]

74. Ringburg AN, Polinder S, van Ierland MCP, et al. Prevalence and prognostic factors of disability after major trauma. J Trauma. 2011; 70:916-22. [PubMed: 21045741]

75. Van Loey NE, van Beeck EF, Faber BW, van de Schoot R, Bremer M. Health-Related Quality of Life After Burns: A Prospective Multicentre Cohort Study With 18 Months Follow-Up. J Trauma. 2011 published online Oct 24. DOI:10.1097/TA.0b013e3182199072.

76. GBD 2013 Mortality and Causes of Death Collaborators. Global, regional, and national age-sex specific all-cause and cause-specific mortality for 240 causes of death, 1990-2013: a systematic analysis for the Global Burden of Disease Study 2013. Lancet. 2014 published online Dec 17. DOI:10.1016/S0140-6736(14)61682-2.

77. Australian Bureau of Statistics. National Survey of Mental Health and Wellbeing of Adults 1997. Australian Bureau of Statistics; Canberra:

78. Rastogi, T.; Mathers, C. Global burden of iron deficiency anaemia in the year 2000. World Health Organization; Geneva: 2002.

79. Kates EH, Kates JS. Anemia and polycythemia in the newborn. Pediatr Rev. 2007; 28:33-34. [PubMed: 17197458] 
80. Bishop, YM.; Fienberg, SE.; Holland, PW. Discrete Multivariate Analysis - Theory and Practice. MIT Press; Cambridge, MA: 1975.

81. Pearson, K. Mathematical contributions to the theory of evolution. Dulau and Co; London: 1904.

82. Edmond K, Clark A, Korczak VS, Sanderson C, Griffths UK, Rudan I. Global and regional risk of disabling sequelae from bacterial meningitis: a systematic review and meta-analysis. Lancet Infect Dis. 2010; 10:317-28. [PubMed: 20417414]

83. Bluestone CD. Epidemiology and pathogenesis of chronic suppurative otitis media: implications for prevention and treatment. Int J Pediatr Otorhinolaryngol. 1998; 42:207-23. [PubMed: 9466224]

84. Minja BM, Machemba A. Prevalence of otitis media, hearing impairment and cerumen impaction among school children in rural and urban Dar es Salaam, Tanzania. Int J Pediatr Otorhinolaryngol. 1996; 37:29-34. [PubMed: 8884404]

85. Salomon JA, Vos T, Hogan DR, et al. Common values in assessing health outcomes from disease and injury: disability weights measurement study for the Global Burden of Disease Study 2010. Lancet. 2012; 380:2129-43. [PubMed: 23245605]

86. Salomon JA. New disability weights for the global burden of disease. Bull World Health Organ. 2010; 88:879. [PubMed: 21124707]

87. Haagsma JA, Noordhout C, Polinder S, et al. The European disability weights study: assessing disability weights based on the responses of 30,660 people from four European countries. Popul Health Metr. 2015; 13:10.

88. Salomon JA, Haagsma JA, Davis A, et al. Disability weights for the Global Burden of Disease 2013 Study. Lancet Global Health. in press.

89. Aregawi, M.; Cibulskis, RE.; Otten, M.; Williams, R. World malaria report 2009. World Health Organization; Geneva: 2009. Chapter 3 Interventions to control malaria.

90. Baltussen R, Knai C, Sharan M. Iron fortification and iron supplementation are cost-effective interventions to reduce iron deficiency in four subregions of the world. J Nutr. 2004; 134:2678-84. [PubMed: 15465766]

91. Casey GJ, Montresor A, Cavalli-Sforza LT, et al. Elimination of iron deficiency anemia and soil transmitted helminth infection: evidence from a fifty-four month iron-folic acid and de-worming program. PLoS Negl Trop Dis. 2013; 7:e2146. [PubMed: 23593517]

92. Yeung CA. A systematic review of the efficacy and safety of fluoridation. Evid Based Dent. 2008; 9:39-43. [PubMed: 18584000]

93. Sustainable Development. [accessed Nov 25, 2014] Proposal for sustainable development goals: sustainable development knowledge platform. 2014. published online Nov 25. http:// sustainabledevelopment.un.org/focussdgs.html

94. Llyod, LS. Best Practices for Dengue Prevention and Control in the Americas. Environmental Health Project; Washington, DC: 2003.

95. Desjeux P. The increase in risk factors for leishmaniasis worldwide. Trans R Soc Trop Med Hyg. 2001; 95:239-43. [PubMed: 11490989]

96. Desjeux P. Leishmaniasis: current situation and new perspectives. Comp Immunol Microbiol Infect Dis. 2004; 27:305-18. [PubMed: 15225981]

97. Franke CR, Staubach C, Ziller M, Schlüter H. Trends in the temporal and spatial distribution of visceral and cutaneous leishmaniasis in the state of Bahia, Brazil, from 1985 to 1999. Trans R Soc Trop Med Hyg. 2002; 96:236-41. [PubMed: 12174769]

98. Reithinger R, Dujardin J-C, Louzir H, Pirmez C, Alexander B, Brooker S. Cutaneous leishmaniasis. Lancet Infect Dis. 2007; 7:581-96. [PubMed: 17714672]

99. Hoy D, Geere JA, Davatchi F, Meggitt B, Barrero LH. A time for action: Opportunities for preventing the growing burden and disability from musculoskeletal conditions in low- and middleincome countries. Best Pract Res Clin Rheumatol. 2014; 28:377-93. [PubMed: 25481422]

100. Blagojevic M, Jinks C, Jeffery A, Jordan KP. Risk factors for onset of osteoarthritis of the knee in older adults: a systematic review and meta-analysis. Osteoarthritis Cartilage. 2010; 18:24-33. [PubMed: 19751691]

101. Hart DJ, Spector TD. The relationship of obesity, fat distribution and osteoarthritis in women in the general population: the Chingford Study. J Rheumatol. 1993; 20:331-35. [PubMed: 8474072] 
102. AIHW. Health-care expenditure on arthritis and other musculoskeletal conditions 2008-09. Australian Institute of Health and Welfare; Canberra: 2014.

103. Statistics Norway. Norway survey of living conditions 2002. Statistics Norway; Olso, Norway: 2004.

104. Statistics Norway. Norway survey of living conditions 2005-2006. Statistics Norway; Olso: 2006.

105. Statistics Norway. Norway survey of living conditions 2008-2009. Statistics Norway; Olso: 2009.

106. Statistics Norway. Norway survey of living conditions 2012. Statistics Norway; Olso, Norway: 2013.

107. Andrews G, Issakidis C, Sanderson K, Corry J, Lapsley H. Utilising survey data to inform public policy: comparison of the cost-effectiveness of treatment of ten mental disorders. Br J Psychiatry. 2004; 184:526-33. [PubMed: 15172947]

108. Vos T, Haby MM, Barendregt JJ, Kruijshaar M, Corry J, Andrews G. The burden of major depression avoidable by longer-term treatment strategies. Arch Gen Psychiatry. 2004; 61:1097103. [PubMed: 15520357]

109. Devries KM, Mak JYT, García-Moreno C, et al. Global health. The global prevalence of intimate partner violence against women. Science. 2013; 340:1527-28. [PubMed: 23788730]

110. Forero R, McLellan L, Rissel C, Bauman A. Bullying behaviour and psychosocial health among school students in New South Wales, Australia: cross sectional survey. BMJ. 1999; 319:344-48. [PubMed: 10435953]

111. Hansen AM, Hogh A, Persson R, Karlson B, Garde AH, Ørbaek P. Bullying at work, health outcomes, and physiological stress response. J Psychosom Res. 2006; 60:63-72. [PubMed: 16380312]

112. Hillberg T, Hamilton-Giachritsis C, Dixon L. Review of meta-analyses on the association between child sexual abuse and adult mental health difficulties: a systematic approach. Trauma Violence Abuse. 2011; 12:38-49. [PubMed: 21288934]

113. Norman RE, Byambaa M, De R, Butchart A, Scott J, Vos T. The long-term health consequences of child physical abuse, emotional abuse, and neglect: a systematic review and meta-analysis. PLoS Med. 2012; 9:e1001349. [PubMed: 23209385]

114. Degenhardt L, Bucello C, Calabria B, et al. GBD illicit drug use writing group. What data are available on the extent of illicit drug use and dependence globally? Results of four systematic reviews. Drug Alcohol Depend. 2011; 117:85-101. [PubMed: 21377813]

115. Cheng H, Deng F, Xiong W, Phillips MR. Prevalence of alcohol use disorders in mainland China: a systematic review. Addiction. 2015 published online Feb 10. DOI:10.1111/add.12876.

116. Pescosolido BA, Martin JK, Long JS, Medina TR, Phelan JC, Link BG. A disease like any other"? A decade of change in public reactions to schizophrenia, depression, and alcohol dependence. Am J Psychiatry. 2010; 167:1321-30. [PubMed: 20843872]

117. Schomerus G, Lucht M, Holzinger A, Matschinger H, Carta MG, Angermeyer MC. The stigma of alcohol dependence compared with other mental disorders: a review of population studies. Alcohol Alcohol. 2011; 46:105-12. [PubMed: 21169612]

118. Schomerus G, Corrigan PW, Klauer T, Kuwert P, Freyberger HJ, Lucht M. Self-stigma in alcohol dependence: consequences for drinking-refusal self-efficacy. Drug Alcohol Depend. 2011; 114:12-17. [PubMed: 20933344]

119. Mathers CD, Iburg KM, Begg S. Adjusting for dependent comorbidity in the calculation of healthy life expectancy. Popul Health Metr. 2006; 4:4. [PubMed: 16620383]

120. van Baal PH, Hoeymans N, Hoogenveen RT, de Wit GA, Westert GP. Disability weights for comorbidity and their influence on health-adjusted life expectancy. Popul Health Metr. 2006; 4:1. [PubMed: 16606448] 


\section{Panel: Research in context}

\section{Systematic review}

The GBD 2013 assessment of morbidity is a major improvement in the evidence base compared with GBD 2010 through the inclusion of new data from surveys, disease registries, and hospital inpatient and outpatient registrations. GBD 2013 also benefits from improvements in the Bayesian meta-regression tool DisMod-MR. The fifty-fold increase in computational speed allowed consistent estimation of prevalence and incidence for each country and time period.

\section{Interpretation}

This study provides a comprehensive description of morbidity levels and patterns worldwide. Because the study provides a complete re-analysis of trends for each cause from 1990 to 2013, it supersedes the results of the GBD 2010 study. This is the first time that country-specific results for all 188 countries with populations of more than 50000 people have been comprehensively published. In all countries, the share of disability in total burden is increasing because of ageing populations and a slower decline in disability rates compared to the decline in mortality. 


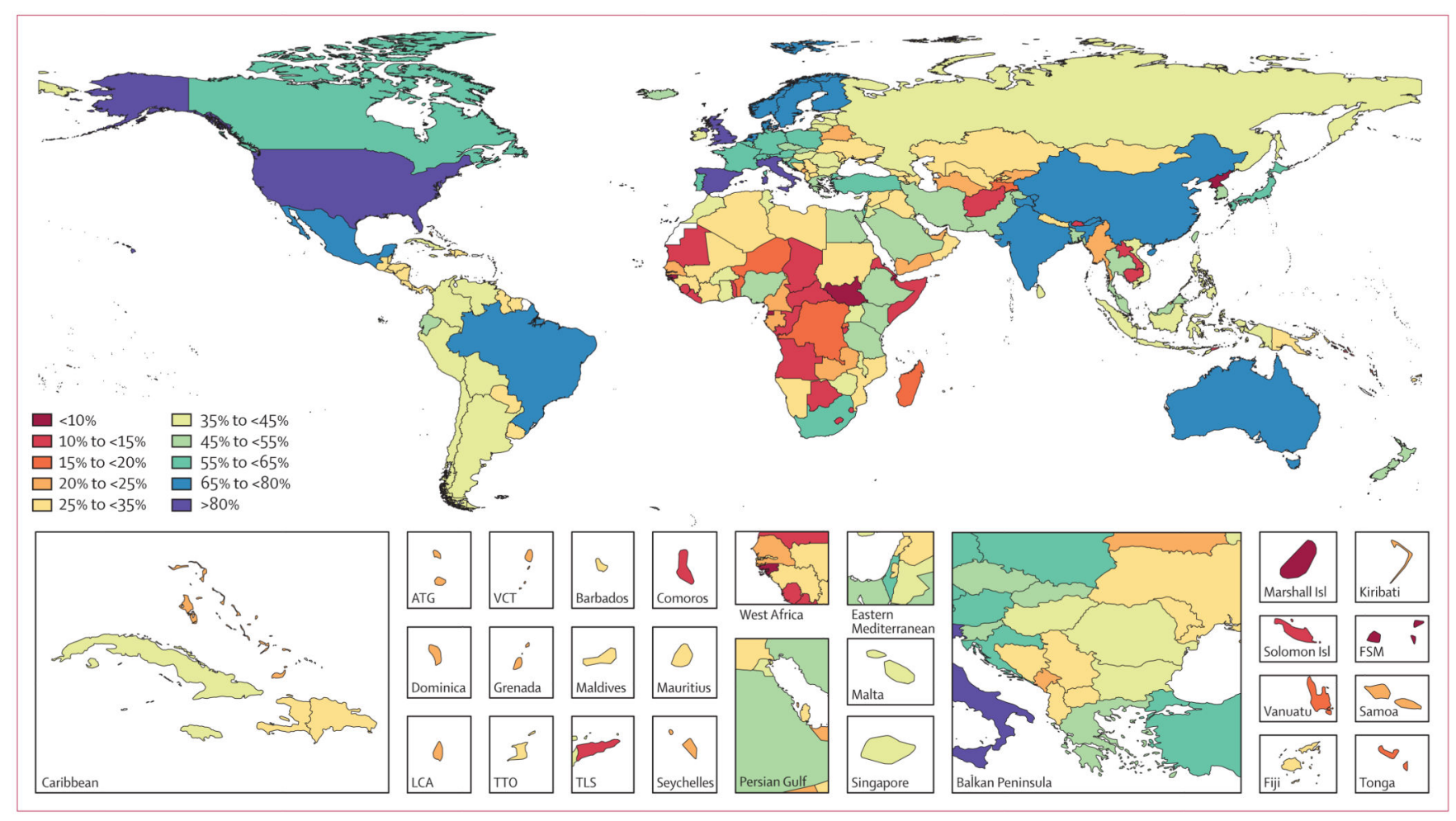

Figure 1. Percentage of causes with data available between 1990 and 2013 for 188 countries ATG=Antigua and Barbuda. FSM=Federated States of Micronesia. LCA=Saint Lucia. TLS=Timor-Leste. TTO=Trinidad and Tobago. VCT=Saint Vincent and the Grenadines. Isl=Islands. 
Number of sequelae

$\square 0 \square 1 \square 2 \square 3 \square 4 \square 5 \square 6 \square 7 \square 8 \square 9 \square \geq 10$

1990

Male population
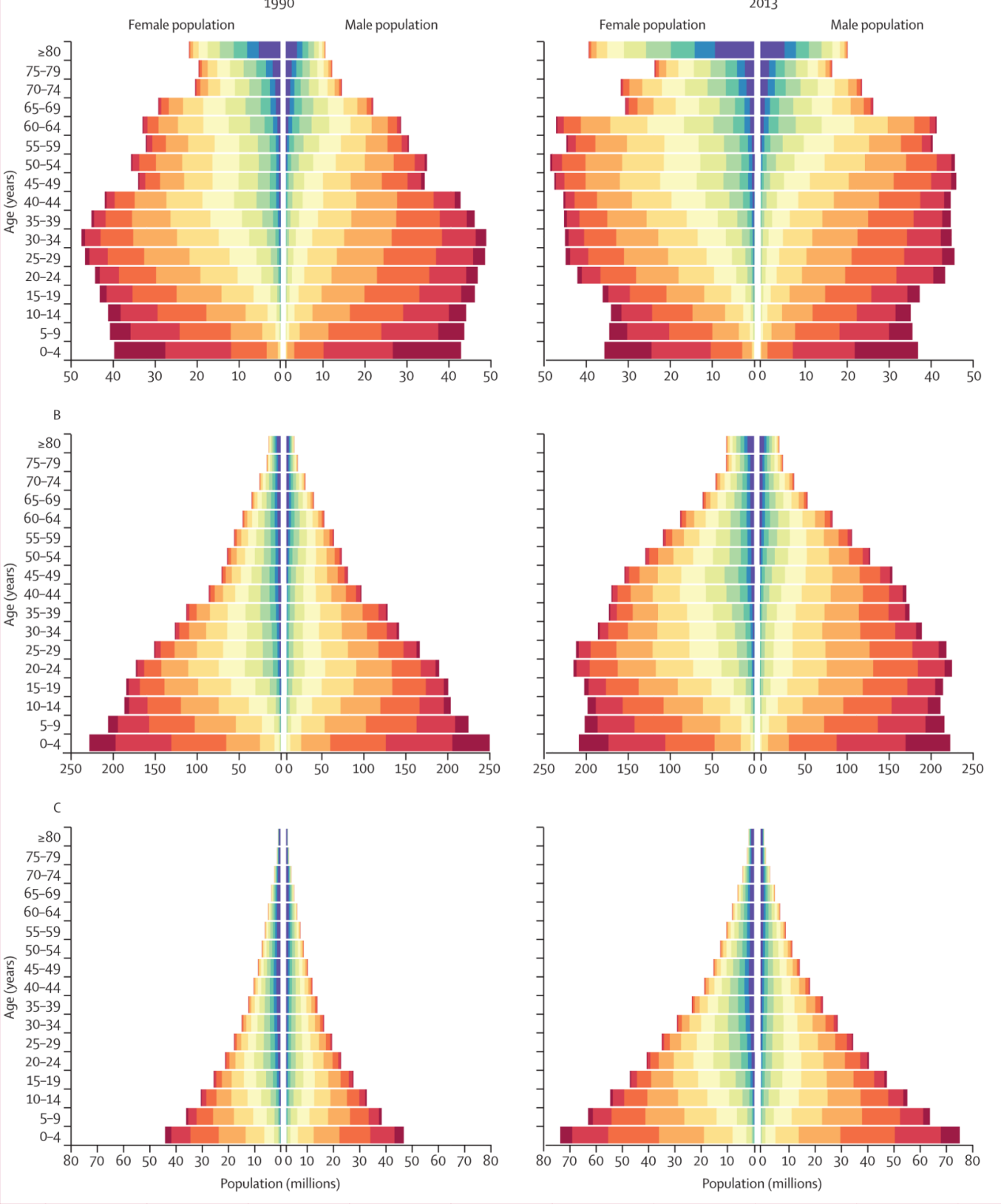

Figure 2. Population pyramids for developed countries (A), developing countries (excluding subSaharan Africa) (B), and sub-Saharan African countries (C) with individuals grouped by number of sequelae, 1990 and 2013 


\begin{tabular}{|c|c|c|c|c|c|c|}
\hline $\begin{array}{l}\text { Mean YLDs } \\
\times 1000\end{array}$ & $\begin{array}{l}\text { Mean rank } \\
(95 \% \text { UI) }\end{array}$ & 1990 leading causes & 2013 leading causes & $\begin{array}{l}\text { Mean rank } \\
(95 \% \text { UI) }\end{array}$ & $\begin{array}{l}\text { Mean YLDs } \\
(\times 1000)\end{array}$ & $\begin{array}{l}\text { Median percentage } \\
\text { change }\end{array}$ \\
\hline 46068 & $1 \cdot 3(1-2)$ & 1 Low back pain & 1 Low back pain & $1 \cdot 0(1-1)$ & 72318 & $57 \%$ (53 to 61$)$ \\
\hline 40079 & $2 \cdot 0(1-3)$ & 2 Iron-deficiency anaemia & 2 Major depression & $2 \cdot 1(2-4)$ & 51784 & $53 \%$ (49 to 59 ) \\
\hline 33711 & $2 \cdot 8(1-4)$ & 3 Major depression & 3 Iron-deficiency anaemia & $3.6(2-6)$ & 36663 & $-9 \%(-10$ to -7$)$ \\
\hline 22294 & $4 \cdot 7(4-6)$ & 4 Neck pain & 4 Neck pain & $4 \cdot 3(3-6)$ & 34348 & $54 \%(49$ to 60$)$ \\
\hline 21633 & $5 \cdot 1(3-7)$ & 5 Other hearing loss & 5 Other hearing loss & $5 \cdot 3(3-9)$ & 32580 & $51 \%(45$ to 55$)$ \\
\hline 19805 & $5 \cdot 8(4-8)$ & 6 Migraine & 6 Migraine & $6 \cdot 6(3-10)$ & 28898 & $46 \%$ (41 to 50$)$ \\
\hline 17180 & $6 \cdot 9(4-9)$ & 7 Anxiety disorders & 7 Diabetes & $6.7(5-9)$ & 29518 & $136 \%(127$ to 144$)$ \\
\hline 15151 & $7 \cdot 9(6-10)$ & 8 COPD & 8 COPD & $7 \cdot 8(4-10)$ & 26131 & $72 \%$ (67 to 79$)$ \\
\hline 12672 & $9 \cdot 5(7-12)$ & 9 Other musculoskeletal & 9 Anxiety disorders & $8 \cdot 5(5-10)$ & 24356 & $42 \%$ (36 to 47) \\
\hline 12533 & $9 \cdot 5(8-11)$ & 10 Diabetes & 10 Other musculoskeletal & $9 \cdot 2(7-10)$ & 22644 & $79 \%$ (75 to 83$)$ \\
\hline 10337 & $11 \cdot 6(10-13)$ & 11 Falls & 11 Schizophrenia & $11 \cdot 5(11-15)$ & 15204 & $52 \%(50$ to 54$)$ \\
\hline 9995 & $12 \cdot 0(9-16)$ & 12 Schizophrenia & 12 Falls & $12 \cdot 7(12-14)$ & 12818 & $23 \%$ (14 to 35$)$ \\
\hline 8048 & $14.7(12-19)$ & 13 Asthma & 13 Osteoarthritis & $12 \cdot 8(11-15)$ & 12811 & $75 \%(73$ to 78$)$ \\
\hline 7831 & $15 \cdot 5(10-23)$ & 14 Refraction and accommodation & 14 Refraction and accommodation & $15 \cdot 5(11-22)$ & 11257 & $44 \%(40$ to 47$)$ \\
\hline 7362 & $16 \cdot 2(13-20)$ & 15 Diarrhoeal diseases & 15 Asthma & $16 \cdot 1(12-21)$ & 10596 & $32 \%(29$ to 35$)$ \\
\hline 7307 & $16 \cdot 4(14-19)$ & 16 Osteoarthritis & 16 Dysthymia & $17 \cdot 4(14-21)$ & 9849 & $55 \%(52$ to 57$)$ \\
\hline 6780 & $18 \cdot 5(14-24)$ & 17 Dermatitis & 17 Bipolar disorder & $17.5(12-25)$ & 9911 & $49 \%$ (46 to 53$)$ \\
\hline 7491 & $18 \cdot 8(8-36)$ & 18 War and legal intervention & 18 Medication overuse headache & $17 \cdot 8(12-27)$ & 9846 & $120 \%(109$ to 134$)$ \\
\hline 6643 & $18 \cdot 8(13-26)$ & 19 Bipolar disorder & 19 Other mental and substance & $18 \cdot 5(14-24)$ & 9257 & $52 \%(50$ to 54$)$ \\
\hline 6368 & $19 \cdot 7(15-24)$ & 20 Dysthymia & 20 Dermatitis & $18.8(15-25)$ & 9278 & $37 \%$ (35 to 39) \\
\hline 6076 & $20 \cdot 6(15-25)$ & 21 Other mental and substance & 21 Alzheimer's disease & $22 \cdot 2(18-26)$ & 7774 & $92 \%$ (85 to 99) \\
\hline 5699 & $22 \cdot 1(17-26)$ & 22 Alcohol use disorders & 22 Alcohol use disorders & $23.0(18-28)$ & 7654 & $34 \%(32$ to 37$)$ \\
\hline 5827 & $22 \cdot 9(12-38)$ & 23 Acne vulgaris & 23 Epilepsy & $23 \cdot 2(18-30)$ & 7544 & $41 \%$ (28 to 57$)$ \\
\hline 5365 & $23 \cdot 5(18-29)$ & 24 Epilepsy & 24 Edentulism & $25.9(21-31)$ & 6856 & $46 \%$ (43 to 48 ) \\
\hline 5288 & $23 \cdot 9(17-31)$ & 25 Conduct disorder & 25 Diarrhoeal diseases & $26 \cdot 1(23-30)$ & 6854 & $-7 \%(-9$ to -5$)$ \\
\hline & & $\begin{array}{l}26 \text { Edentulism } \\
27 \text { Medication overuse headache } \\
28 \text { Alzheimer's disease }\end{array}$ & $\begin{array}{l}26 \text { Acne vulgaris } \\
29 \text { Conduct disorder } \\
52 \text { War and legal intervention }\end{array}$ & \multicolumn{3}{|c|}{$\begin{array}{l}\square \text { Communicable, maternal, neonatal } \\
\text { and nutritional disorders } \\
\square \text { Non-communicable diseases } \\
\square \text { Injuries }\end{array}$} \\
\hline
\end{tabular}

Figure 3. Top 25 causes of global YLDs in 1990 and 2013

YLD=years lived with disability. UI=uncertainty interval. $\mathrm{COPD}=$ chronic obstructive pulmonary disease. 


\begin{tabular}{|c|c|c|c|c|c|c|c|c|c|c|}
\hline & 1 & 2 & 3 & 4 & 5 & 6 & 7 & 8 & 9 & 10 \\
\hline Global & Back pain & MDD & Iron & Neck pain & Hearing & Migraine & \begin{tabular}{|l} 
Diabetes \\
\end{tabular} & COPD & Anxiety & Other MSK \\
\hline Developed countries & Back pain & MDD & Neck pain & Other MSK & Hearing & Diabetes & \begin{tabular}{|l} 
Migraine \\
\end{tabular} & Fals & Anxiety & COPD \\
\hline Developing countries & Backpain & MDD & Iron & Neck pain & Hearing & Migraine & Diabetes & COPD & Anxiety & Other MSK \\
\hline High-income countries & Back pain & Neck pain & MDD & Other MSK & Diabetes & Hearing & Anxiety & Falls & Migraine & COPD \\
\hline Australasia & Back pain & MDD & Other MSK & Neck pain & Migraine & Anxiety & Asthma & COPD & Hearing & Diabetes \\
\hline Australia & Back pain & MDD & Other MSK & Neck pain & Migraine & Anxiety & COPD & Asthma & Hearing & Diabetes \\
\hline New Zealand & Back pain & MDD & Neck pain & Anxiety & Other MSK & Asthma & COPD & Hearing & Diabetes & Migraine \\
\hline High-income Asia Pacific & Backpain & Other MSK & Neck pain & Diabetes & MDD & Hearing & Iron & Migraine & Falls & Osteoarthritis \\
\hline Brunei & Back pain & Neck pain & MDD & Iron & Other MSK & Migraine & Anxiety & Diabetes & Schizophrenia & Dermatitis \\
\hline Japan & Back pain & Other MSK & Diabetes & Neck pain & MDD & Hearing & Falls & Iron & Altheimer & Osteoarthritis \\
\hline Singapore & MDD & Diabetes & Other MSK & Neck pain & Back pain & Iron & Hearing & Falls & Anxiety & Schizophrenia \\
\hline South Korea & Back pain & Neck pain & Other MSK & Migraine & Diabetes & Iron & MDD & Hearing & Anxiety & Falls \\
\hline High-income North America & Back pain & Other MSK & MDD & Anxiety & COPD & Diabetes & Neck pain & Hearing & Falls & Migraine \\
\hline Canada & Back pain & Other MSK & Neck pain & MDD & Hearing & Diabetes & Migraine & Anxiety & Asthma & Falls \\
\hline USA & Back pain & MDD & Other MSK & Anxiety & COPD & Diabetes & Neck pain & Hearing & Falls & Migraine \\
\hline Southern Latin America & Backpain & MDD & Neck pain & Anxiety & Other MSK & COPD & Hearing & Asthma & Diabetes & Iron \\
\hline Argentina & Back pain & MDD & Neck pain & Anxiety & Other MSK & COPD & Hearing & Iron & Diabetes & Asthma \\
\hline Chile & Back pain & MDD & Neck pain & Anxiety & Other MSK & COPD & Epilepsy & Migraine & Hearing & Asthma \\
\hline Uruguay & Backpain & MDD & Neck pain & Anxiety & Other MSK & COPD & Hearing & Asthma & Iron & Diabetes \\
\hline Western Europe & Back pain & Neck pain & Falls & MDD & Hearing & Diabetes & Migraine & Anxiety & Other MSK & Atheimer \\
\hline Andorra & Back pain & Neck pain & MDD & Falls & Hearing & Migraine & Anxiety & Other MSK & COPD & Atheimer \\
\hline Austria & Neck pain & Backpain & Migraine & Fals & MDD & Hearing & Diabetes & Anxiety & Other MSK & COPD \\
\hline Belgium & Back pain & Neck pain & Falls & MDD & Diabetes & Migraine & COPD & Hearing & Altheimer & Anxiety \\
\hline Cyprus & Back pain & Neck pain & MDD & Diabetes & Migraine & Anxiety & Falls & Hearing & Other MSK & COPD \\
\hline Denmark & Back pain & Neck pain & MDD & Falls & COPD & Other MSK & Anxiety & Atheimer & Hearing & Migraine \\
\hline Finland & Back pain & Falls & Neck pain & MDD & Diabetes & Hearing & Migraine & Oth unint & Alzheimer & Asthma \\
\hline France & Back pain & MDD & Neck pain & Fals & Anxiety & Hearing & Migraine & Other MSK & Diabetes & Atheimer \\
\hline Germany & Back pain & Hearing & Neck pain & Fals & Diabetes & MDD & Anxiety & Other MSK & Migraine & Atheimer \\
\hline Greece & Back pain & Neck pain & Falls & MDD & Hearing & Diabetes & Other MSK & Anxiety & COPD & Azheimer \\
\hline keland & Back pain & Neck pain & Diabetes & MDD & Fals & Migraine & COPD & Anxiety & Hearing & Other MSK \\
\hline Ireand & MDD & Neck pain & Back pain & Anxiety & Migraine & Falls & Asthma & Diabetes & Hearing & Other MSK \\
\hline Israel & Back pain & MDD & Neck pain & Migraine & Fals & Hearing & Other MSK & Diabetes & Iron & COPD \\
\hline Italy & Back pain & Neck pain & Migraine & Fals & Hearing & MDD & Other MSK & Atheimer & Anxiety & COPD \\
\hline Luxembourg & Back pain & Neck pain & Migraine & MDD & Falls & Diabetes & Hearing & Anxiety & Other MSK & COPD \\
\hline Malta & Back pain & Neck pain & Diabetes & MDD & Falls & Migraine & Hearing & Anxiety & Other MSK & COPD \\
\hline Netherlands & Back pain & Neck pain & Anxiety & Migraine & MDD & Diabetes & Hearing & Other MSK & Dermatitis & COPD \\
\hline Norway & Back pain & Neck pain & Anxiety & MDD & Falls & Diabetes & Hearing & Other MSK & Migraine & Atheimer \\
\hline Portugal & Back pain & MDD & Neck pain & Diabetes & Hearing & Migraine & Anxiety & Other MSK & Asthma & Falls \\
\hline Spain & Diabetes & \begin{tabular}{|l|} 
Back pain \\
\end{tabular} & Neck pain & MDD & Falls & Hearing & Migraine & Other MSK & COPD & Anxiety \\
\hline Sweden & Back pain & Falls & MDD & Neck pain & Hearing & COPD & Anxiety & Migraine & Other MSK & Asthma \\
\hline Switzerland & Back pain & Falls & Neck pain & COPD & MDD & Hearing & Migraine & Anxiety & Diabetes & Atheimer \\
\hline UK & Backpain & Neck pain & Falls & MDD & Diabetes & Hearing & Migraine & Anxiety & Other MSK & COPD \\
\hline England & Back pain & Neck pain & Falls & MDD & Diabetes & Hearing & Migraine & Anxiety & Other MSK & COPD \\
\hline Northern Ireland & Back pain & MDD & Neck pain & Falls & Diabetes & Migraine & Hearing & Anxiety & Other MSK & COPD \\
\hline Scotand & Back pain & Neck pain & Diabetes & Falls & Hearing & Migraine & Asthma & Other MSK & MDD & Anxiety \\
\hline Wales & Back pain & MDD & Neck pain & Falls & Hearing & Diabetes & Migraine & Anxiety & Other MSK & COPD \\
\hline Central Europe, eastem Europe, and central Asia & Back pain & MDD & Hearing & Migraine & Neck pain & Diabetes & Falls & \begin{tabular}{|l|} 
Osteoarthritis \\
\end{tabular} & Other MSK & Med Head \\
\hline Central Asia & Back pain & MDD & Diabetes & Migraine & Iron & Neck pain & Hearing & Falls & Anxiety & Osteoarthritis \\
\hline Armenia & Backpain & MDD & Diabetes & Hearing & Migraine & Falls & Neck pain & Iron & Osteoarthritis & Anxiety \\
\hline Azerbaijan & Back pain & MDD & Diabetes & Migraine & Iron & Falls & Neck pain & Hearing & Anxiety & Osteoarthritis \\
\hline Georgia & Diabetes & MDD & Hearing & Back pain & Falls & Migraine & Neck pain & Osteoarthritis & COPD & Other MSK \\
\hline Kazakhstan & Back pain & MDD & Diabetes & Migraine & Hearing & Neck pain & Iron & Falls & Anxiety & Osteoarthritis \\
\hline Kyrgyzstan & MDD & \begin{tabular}{|l|} 
Back pain \\
\end{tabular} & Migraine & Diabetes & Iron & Neck pain & Hearing & Falls & Anxiety & Osteoarthritis \\
\hline Mongolia & MDD & Back pain & Migraine & Diabetes & Neck pain & Hearing & Iron & Fals & Anxiety & Akohol \\
\hline Tajikistan & MDD & Back pain & Iron & Migraine & Diabetes & Falls & Neck pain & Hearing & Anxiety & Schizophrenia \\
\hline Turkmenistan & MDD & Back pain & Migraine & Diabetes & Iron & Neck pain & Hearing & Fals & Anxiety & Schizophrenia \\
\hline Uzbekistan & MDD & Back pain & Migraine & Diabetes & Iron & Neck pain & Hearing & Fals & Anxiety & Schizophrenia \\
\hline Central Europe & Back pain & Falls & MDD & Hearing & Diabetes & Neck pain & Migraine & Osteoarthritis & COPD & Iron \\
\hline Albania & Back pain & Falls & MDD & Iron & Migraine & Hearing & Diabetes & Neck pain & Anxiety & Osteoarthritis \\
\hline Bosnia and Herzegovina & Back pain & Falls & MDD & Hearing & Diabetes & Neck pain & Migraine & Osteoarthritis & War & COPD \\
\hline Bulgaria & Backpain & Falls & Diabetes & Hearing & MDD & Neck pain & Osteoarthritis & Migraine & COPD & Anxiety \\
\hline Croatia & Back pain & Hearing & MDD & Diabetes & Falls & Neck pain & Migraine & Osteoarthritis & COPD & Anxiety \\
\hline Grech Republic & Back pain & Falls & MDD & Hearing & Diabetes & Neck pain & Migraine & Osteoarthritis & COPD & Anxiety \\
\hline Hungary & Back pain & Falls & MDD & Hearing & Diabetes & Neck pain & Migraine & Osteoarthritis & COPD & Anxiety \\
\hline Macedonia & Back pain & Falls & MDD & Hearing & Diabetes & Neck pain & Migraine & Osteoarthritis & Iron & COPD \\
\hline Montenegro & Back pain & Falls & MDD & Hearing & Diabetes & Neck pain & Migraine & Osteoarthritis & Iron & COPD \\
\hline Poland & Back pain & Falls & MDD & Hearing & Diabetes & Neck pain & Migraine & Osteoarthritis & COPD & Anxiety \\
\hline Romania & Backpain & Falls & MDD & Hearing & Diabetes & Neck pain & Migraine & Osteoarthritis & Iron & COPD \\
\hline Serbia & Back pain & MDD & Hearing & Diabetes & Falls & Neck pain & Migraine & Osteoarthritis & COPD & Iron \\
\hline
\end{tabular}

Lancet. Author manuscript; available in PMC 2015 September 07. 


\begin{tabular}{|c|c|c|c|c|c|c|c|c|c|c|}
\hline & 1 & 2 & 3 & 4 & 5 & 6 & 7 & 8 & 9 & 10 \\
\hline $\begin{array}{l}\text { Slovakia } \\
\text { Slovenia }\end{array}$ & $\begin{array}{l}\text { Back pain } \\
\text { Back pain }\end{array}$ & $\begin{array}{l}\text { Fals } \\
\text { Fals }\end{array}$ & $\begin{array}{l}\text { MDD } \\
\text { Hearing }\end{array}$ & $\begin{array}{l}\text { Hearing } \\
\text { MDD }\end{array}$ & $\begin{array}{l}\text { Diabetes } \\
\text { Diabetes }\end{array}$ & $\begin{array}{l}\text { Neck pain } \\
\text { Neck pain }\end{array}$ & $\begin{array}{l}\text { Migraine } \\
\text { Migraine }\end{array}$ & $\begin{array}{l}\text { Osteoarthritis } \\
\text { Osteoarthritis }\end{array}$ & $\begin{array}{l}\text { COPD } \\
\text { COPD }\end{array}$ & $\begin{array}{l}\text { Anxiety } \\
\text { Anxiety }\end{array}$ \\
\hline Eastern Europe & Back pain & MDD & Hearing & Migraine & Neck pain & Med head & Other MSK & Diabetes & Osteoarthritis & Akohol \\
\hline Belarus & Back pain & MDD & Hearing & Migraine & Neck pain & Osteoarthritis & Med head & Other MSK & \begin{tabular}{|l|} 
Diabetes \\
\end{tabular} & Akohol \\
\hline Estonia & MDD & Back pain & Hearing & Migraine & Neck pain & Diabetes & Osteoarthritis & Other MSK & Med Head & Akohol \\
\hline Latvia & Back pain & Hearing & MDD & Migraine & Neck pain & Osteoarthritis & Diabetes & Other MSK & Med Head & COPD \\
\hline Lithuania & Back pain & MDD & Hearing & Migraine & Neck pain & Osteoarthritis & Diabetes & Other MSK & Med Head & COPD \\
\hline Moldova & Back pain & MDD & Hearing & Migraine & Neck pain & Iron & Diabetes & Osteoarthritis & Med Head & Other MSK \\
\hline Russia & Back pain & MDD & Hearing & Migraine & Neck pain & Med head & Other MSK & Diabetes & Osteoarthritis & Akohol \\
\hline Ukraine & Back pain & MDD & Hearing & Migraine & Neck pain & Osteoarthritis & Diabetes & Other MSK & Med head & COPD \\
\hline Latin America and Caribbean & Back pain & MDD & Anxiety & Diabetes & Hearing & Other MSK & Iron & Migraine & Neck pain & Asthma \\
\hline Andean Latin America & MDD & Back pain & Anxiety & Iron & Migraine & Hearing & Neck pain & COPD & Asthma & Other MSK \\
\hline Bolivia & MDD & Iron & Back pain & Anxiety & Migraine & Hearing & Neck pain & Other MSK & COPD & Asthma \\
\hline Ecuador & MDD & Back pain & Iron & Anxiety & Hearing & Migraine & Neck pain & COPD & Other MSK & Asthma \\
\hline Peru & MDD & Back pain & Anxiety & Migraine & Iron & Hearing & Asthma & War & Neck pain & COPD \\
\hline Caribbean & MDD & Iron & Diabetes & Anxiety & Back pain & Hearing & Neck pain & Migraine & COPD & Other MSK \\
\hline Antigua and Barbuda & MDD & Anxiety & Diabetes & Iron & Back pain & Hearing & Migraine & Neck pain & COPD & Other MSK \\
\hline Barbados & MDD & Diabetes & Back pain & Hearing & Anxiety & Asthma & Iron & COPD & Other MSK & Neck pain \\
\hline Belize & MDD & Iron & Anxiety & Diabetes & Back pain & Migraine & Hearing & Neck pain & COPD & Other MSK \\
\hline Cuba & Diabetes & MDD & Hearing & Anxiety & Back pain & Asthma & COPD & Iron & Neck pain & other MSK \\
\hline Dominica & MDD & Diabetes & Anxiety & Back pain & Hearing & Iron & Migraine & Neck pain & COPD & Other MSK \\
\hline Dominican Republic & MDD & Back pain & Iron & Anxiety & Diabetes & Hearing & Migraine & Neck pain & COPD & Other MSK \\
\hline Grenada & MDD & Iron & Anxiety & Back pain & Diabetes & Hearing & Migraine & Neck pain & COPD & Other MSK \\
\hline Guyana & Iron & MDD & Anxiety & Back pain & Diabetes & Migraine & Hearing & Neck pain & COPD & Other MSK \\
\hline Haiti & Iron & MDD & Anxiety & Disaster & Back pain & Hearing & Migraine & Neck pain & Diabetes & COPD \\
\hline Jamaica & MDD & Iron & Anxiety & Back pain & Diabetes & Hearing & Migraine & Neck pain & COPD & Other MSK \\
\hline Saint Lucia & MDD & Diabetes & Anxiety & Back pain & Hearing & Iron & Neck pain & COPD & Migraine & Other MSK \\
\hline Saint Vincent and the Grenadines & MDD & Diabetes & Anxiety & Back pain & Iron & Hearing & Migraine & Neck pain & COPD & Other MSK \\
\hline Suriname & MDD & Iron & Anxiety & Diabetes & Back pain & Hearing & Migraine & Neck pain & COPD & Other MSK \\
\hline The Bahamas & MDD & Diabetes & Anxiety & Back pain & Hearing & Iron & Migraine & COPD & Neck pain & Other MSK \\
\hline Trinidad and Tobago & MDD & Diabetes & Anxiety & Back pain & Hearing & Iron & Other MSK & COPD & Migraine & Neck pain \\
\hline Central Latin America & MDD & Back pain & Diabetes & Anxiety & Hearing & Iron & Other MSK & Migraine & Neck pain & COPD \\
\hline Colombia & MDD & Back pain & Anxiety & Hearing & Diabetes & Other MSK & Asthma & Migraine & COPD & Iron \\
\hline Costa Rica & MDD & Back pain & Asthma & Hearing & Anxiety & Other MSK & Diabetes & Neck pain & COPD & Migraine \\
\hline El Salvador & Back pain & MDD & Iron & Hearing & Anxiety & Asthma & Diabetes & Other MSK & Neck pain & War \\
\hline Guatemala & Iron & Back pain & MDD & Anxiety & War & Hearing & Diabetes & Migraine & Neck pain & COPD \\
\hline Honduras & MDD & Back pain & Iron & Anxiety & Hearing & Diabetes & Asthma & Neck pain & Migraine & COPD \\
\hline Mexico & MDD & Back pain & Diabetes & Hearing & Other MSK & Anxiety & Iron & Neck pain & Migraine & COPD \\
\hline Nicaragua & War & MDD & Back pain & Iron & Diabetes & Anxiety & Hearing & Asthma & Migraine & Neck pain \\
\hline Panama & Back pain & MDD & Diabetes & Hearing & Anxiety & Asthma & Iron & Other MSK & Neck pain & Migraine \\
\hline Venezuela & Back pain & MDD & Diabetes & Hearing & Anxiety & Other MSK & Migraine & Neck pain & COPD & Asthma \\
\hline Tropical Latin America & Back pain & MDD & Anxiety & Diabetes & Hearing & Other MSK & Asthma & Neck pain & Migraine & COPD \\
\hline Brazil & Back pain & MDD & Anxiety & Diabetes & Hearing & Other MSK & Asthma & Neck pain & Migraine & COPD \\
\hline Paraguay & Back pain & Anxiety & MDD & Hearing & Other MSK & Migraine & Diabetes & Neck pain & Asthma & Iron \\
\hline Southeast Asia, east Asia, and Oceania & Back pain & Neck pain & MDD & Hearing & Diabetes & Iron & Schizophrenia & Other MSK & COPD & \begin{tabular}{|l} 
Migraine \\
\end{tabular} \\
\hline East Asia & Back pain & Neck pain & MDD & Diabetes & Hearing & Other MSK & Schizophrenia & Iron & COPD & Anxiety \\
\hline China & Back pain & Neck pain & MDD & Diabetes & Hearing & Other MSK & Schizophrenia & Iron & COPD & Anxiety \\
\hline North Korea & Back pain & Neck pain & Iron & Hearing & MDD & Schizophrenia & Diabetes & Other MSK & COPD & Anxiety \\
\hline Taiwan & Back pain & Neck pain & Hearing & Diabetes & Anxiety & Other MSK & COPD & Migraine & Schizophrenia & MDD \\
\hline Oceania & Back pain & Iron & MDD & Diabetes & Hookworm & Neck pain & Hearing & Migraine & Anxiety & COPD \\
\hline Federated States of Micronesia & Back pain & Iron & Diabetes & MDD & Hearing & Neck pain & Anxiety & Migraine & COPD & Dermatitis \\
\hline $\mathrm{Fiji}$ & Back pain & Diabetes & MDD & Hearing & Neck pain & Iron & COPD & Other MSK & Migraine & Anxiety \\
\hline Kiribati & Trichuria & Back pain & Iron & MDD & Neck pain & Hearing & Diabetes & Migraine & RHD & Anxiety \\
\hline Marshall Islands & Back pain & Iron & Trichuria & MDD & Hearing & Neck pain & Diabetes & Anxiety & COPD & Migraine \\
\hline Papua New Guinea & Iron & Back pain & Hookworm & MDD & Diabetes & Neck pain & Hearing & Migraine & Anxiety & Dermatitis \\
\hline Samoa & Back pain & Iron & Hearing & MDD & Diabetes & Neck pain & COPD & Dermatitis & Anxiety & Migraine \\
\hline Solomon Islands & Back pain & Iron & MDD & Hearing & Neck pain & Diabetes & RHD & Anxiety & Migraine & Dermatitis \\
\hline Tonga & Back pain & Iron & Hearing & MDD & Diabetes & Neck pain & COPD & Anxiety & Migraine & Other MSK \\
\hline Vanuatu & Back pain & MDD & Hearing & Neck pain & Iron & Diabetes & Migraine & Anxiety & Thalass trait & COPD \\
\hline Southeast Asia & Back pain & Iron & Hearing & MDD & Migraine & Diabetes & COPD & Neck pain & Schizophrenia & Anxiety \\
\hline Cambodia & War & Iron & Back pain & Hearing & Migraine & MDD & COPD & Neck pain & Anxiety & Schizophrenia \\
\hline Indonesia & Back pain & Iron & Migraine & MDD & Hearing & COPD & Diabetes & Neck pain & Schizophrenia & Anxiety \\
\hline Laos & Iron & Back pain & FB trema & Migraine & Hearing & MDD & COPD & Neck pain & Diabetes & Anxiety \\
\hline Malaysia & Back pain & Diabetes & Anxiety & MDD & COPD & Migraine & Hearing & Neck pain & Trichuria & Iron \\
\hline Maldives & Back pain & Diabetes & Iron & MDD & Migraine & Hearing & COPD & Thalass trait & Neck pain & Anxiety \\
\hline Myanmar & Hearing & Back pain & Migraine & MDD & Iron & COPD & Diabetes & Neck pain & Schizophrenia & Anxiety \\
\hline Philippines & Back pain & Iron & Hearing & MDD & Diabetes & Migraine & COPD & Neck pain & Anxiety & Schizophrenia \\
\hline Srilanka & Back pain & Hearing & Iron & Diabetes & MDD & Migraine & COPD & Neck pain & Asthma & Schizophrenia \\
\hline
\end{tabular}

Lancet. Author manuscript; available in PMC 2015 September 07. 


\begin{tabular}{|c|c|c|c|c|c|c|c|c|c|c|}
\hline & 1 & 2 & 3 & 4 & 5 & 6 & 7 & 8 & 9 & 10 \\
\hline Thailand & Migraine & Hearing & \begin{tabular}{|l|l} 
Diabetes \\
\end{tabular} & MDD & Other MSK & Back pain & COPD & Neck pain & Schizophrenia & Asthma \\
\hline Timor-Leste & Iron & Back pain & Migraine & Hearing & MDD & COPD & Refraction & Neck pain & Anxiety & Diarrhoea \\
\hline Vietnam & Back pain & War & MDD & Hearing & Iron & Migraine & COPD & Neck pain & Schizophrenia & Diabetes \\
\hline South Asia & Back pain & MDD & Iron & Migraine & COPD & Hearing & Neck pain & Diabetes & Anxiety & Refraction \\
\hline Afghanistan & Iron & Back pain & MDD & War & Anxiety & Migraine & Opioids & Neck pain & Diarrhoea & Diabetes \\
\hline Bangladesh & Back pain & Iron & MDD & Migraine & COPD & Anxiety & Hearing & Other MSK & Neck pain & Diabetes \\
\hline Bhutan & Back pain & MDD & Migraine & COPD & Hearing & Iron & Anxiety & Neck pain & Diabetes & Other MSK \\
\hline India & MDD & Back pain & Iron & Migraine & COPD & Hearing & Neck pain & Diabetes & Anxiety & Refraction \\
\hline Nepal & Back pain & Iron & Migraine & COPD & MDD & Hearing & Anxiety & Neck pain & Other MSK & Diabetes \\
\hline Pakistan & MDD & Iron & Back pain & Migraine & COPD & Diabetes & Hearing & Anxiety & Neck pain & Diarrhoea \\
\hline North Africa and Middle East & Back pain & MDD & Iron & Diabetes & Migraine & Neck pain & Anxiety & Opioids & COPD & Hearing \\
\hline Algeria & Back pain & MDD & Iron & Diabetes & Neck pain & Migraine & Anxiety & Opioids & Other MSK & COPD \\
\hline Bahrain & Back pain & Diabetes & MDD & Opioids & Iron & Neck pain & Migraine & Anxiety & COPD & Other MSK \\
\hline Egypt & Back pain & Iron & Diabetes & MDD & Neck pain & Migraine & Anxiety & COPD & Refraction & Other MSK \\
\hline Iran & Back pain & MDD & Neck pain & Diabetes & Anxiety & Migraine & Iron & Opioids & COPD & Med head \\
\hline Iraq & Iron & Back pain & Anxiety & MDD & Diabetes & War & Migraine & Neck pain & COPD & Dermatitis \\
\hline Jordan & MDD & Diabetes & Back pain & Iron & Opioids & Anxiety & Migraine & Neck pain & COPD & NN preterm \\
\hline Kuwait & Diabetes & MDD & Opioids & Iron & Neck pain & Migraine & Anxiety & Back pain & COPD & Asthma \\
\hline Lebanon & Diabetes & MDD & War & Anxiety & Iron & Opioids & Neck pain & Back pain & Migraine & COPD \\
\hline Libya & Back pain & Diabetes & MDD & Iron & Neck pain & Anxiety & Migraine & Opioids & COPD & Hearing \\
\hline Morocco & Back pain & MDD & Iron & Diabetes & Neck pain & Migraine & Anxiety & COPD & Opioids & Other MSK \\
\hline Oman & Back pain & MDD & Diabetes & Opioids & Iron & Migraine & Anxiety & Neck pain & COPD & Asthma \\
\hline Palestine & MDD & Back pain & Iron & Diabetes & Anxiety & Migraine & Neck pain & COPD & Other MSK & Opioids \\
\hline Qatar & Opioids & Back pain & MDD & Diabetes & Neck pain & Migraine & Anxiety & COPD & Schizophrenia & Iron \\
\hline Saudi Arabia & Diabetes & Back pain & MDD & Opioids & Iron & Migraine & Neck pain & Anxiety & Asthma & COPD \\
\hline Sudan & Iron & Back pain & MDD & Anxiety & Neck pain & Migraine & Diabetes & Schisto & Refraction & Diarrhoea \\
\hline Syria & MDD & Back pain & War & Migraine & Diabetes & Iron & Anxiety & Neck pain & Opioids & COPD \\
\hline Tunisia & MDD & Back pain & Diabetes & Neck pain & Iron & Anxiety & Opioids & COPD & Hearing & Other MSK \\
\hline Turkey & Back pain & MDD & Migraine & Diabetes & Iron & Neck pain & COPD & Opioids & Anxiety & Other MSK \\
\hline United Arab Emirates & Back pain & MDD & Opioids & Diabetes & Neck pain & Iron & Anxiety & Migraine & COPD & Schizophrenia \\
\hline Yemen & Iron & Back pain & MDD & Anxiety & Migraine & Neck pain & COPD & Dermatitis & Diarrhoea & Conduct \\
\hline Sub-Saharan Africa & MDD & Iron & Back pain & Oth HIV & Neck pain & Hearing & Schisto & COPD & Anxiety & Malaria \\
\hline Central sub-Saharan Africa & Iron & MDD & Oncho & Back pain & Neck pain & Hearing & Schisto & Malaria & Diarthoea & COPD \\
\hline Angola & MDD & Iron & Back pain & Schisto & Neck pain & Diarrhoea & Malaria & COPD & Migraine & Anxiety \\
\hline Central African Republic & Iron & MDD & Back pain & Oncho & Malaria & Neck pain & COPD & Oth HIV & Hearing & Anxiety \\
\hline Congo & MDD & Iron & Back pain & Neck pain & Malaria & COPD & Schisto & Hearing & Migraine & Anxiety \\
\hline Democratic Republic of the Congo & Iron & MDD & Oncho & Back pain & Hearing & Neck pain & Malaria & Diarrhoea & COPD & Schisto \\
\hline Equatorial Guinea & MDD & Back pain & Iron & Neck pain & Malaria & Oth HIV & COPD & Hearing & Migraine & Anxiety \\
\hline Gabon & MDD & Iron & Back pain & Schisto & Neck pain & Mabria & Oth HIV & COPD & Hearing & Anxiety \\
\hline Eastern sub-Saharan Africa & MDD & Iron & Back pain & Neck pain & Oth HIV & Hearing & Schisto & Anxiety & COPD & Malaria \\
\hline Burundi & MDD & Iron & War & Back pain & Neck pain & Hearing & COPD & Anxiety & Diarrhoea & Migraine \\
\hline Comoros & MDD & Iron & Back pain & Neck pain & Hearing & COPD & Malaria & Anxiety & Migraine & Refraction \\
\hline Dijbouti & MDD & Iron & Back pain & Neck pain & Hearing & COPD & Anxiety & Other MSK & Migraine & Schisto \\
\hline Enitrea & Iron & MDD & War & Back pain & Schisto & Neck pain & Hearing & Anxiety & COPD & \begin{tabular}{|l} 
Migraine \\
\end{tabular} \\
\hline Ethiopia & MDD & Iron & Back pain & Anxiety & Neck pain & Hearing & Schisto & COPD & Refraction & Diarrhoea \\
\hline Kenya & MDD & Iron & Eack pain & OthHIV & Schisto & Neck pain & Hearing & COPD & Anxiety & Migraine \\
\hline Madagascar & MDD & Iron & Hearing & Back pain & Schisto & Neck pain & COPD & Malaría & Anxiety & LF \\
\hline Malawi & MDD & Oth HIV & Iron & Back pain & Malaria & Neck pain & Hearing & COPD & Anxiety & Diarrhoea \\
\hline Mauritius & Back pain & Diabetes & Hearing & MDD & Migraine & COPD & Other MSK & Neck pain & Schizophrenia & Anxiety \\
\hline Mozambique & Oth HIV & MDD & Iron & Back pain & Schisto & Hearing & Neck pain & Malaria & COPD & Anxiety \\
\hline Rwanda & War & MDD & Iron & Back pain & Neck pain & Hearing & COPD & Anxiety & Asthma & Schisto \\
\hline Seychelles & Diabetes & Back pain & Hearing & MDD & Migraine & COPD & Neck pain & Schizophrenia & Anxiety & Other MSK \\
\hline Somalia & MDD & Iron & Back pain & Schisto & Hearing & Neck pain & COPD & Anxiety & War & Diarrhoea \\
\hline South Sudan & MDD & Oncho & Iron & Back pain & Schisto & Hearing & Neck pain & COPD & Anxiety & Refraction \\
\hline Tanzania & MDD & Iron & Back pain & Oth HIV & Neck pain & COPD & Anxiety & Hearing & Mabria & LF \\
\hline Uganda & MDD & Iron & Malaria & Oth HIV & Back pain & War & Hearing & Neck pain & COPD & Diarrhoea \\
\hline Zambia & MDD & Oth HIV & Iron & Migraine & Back pain & Neck pain & Hearing & Schisto & Makria & COPD \\
\hline Southern sub-Saharan Africa & Oth HIV & MDD & Back pain & Neck pain & Iron & COPD & TB & Anxiety & Diabetes & Migraine \\
\hline Botswana & MDD & Oth HIV & Backpain & Iron & Neck pain & Anxiety & COPD & ТВ & Migraine & Hearing \\
\hline Lesotho & Oth HIV & MDD & Iron & Back pain & Neck pain & Anxiety & COPD & ТВ & ID & Migraine \\
\hline Namibia & MDD & Iron & Oth HIV & Back pain & Neck pain & Anxiety & COPD & ТВ & Migraine & ID \\
\hline South Africa & Oth HIV & Back pain & MDD & Neck pain & Iron & COPD & Tв & Anxiety & Diabetes & Migraine \\
\hline Swazland & Oth HIV & MDD & Iron & Neck pain & Back pain & Anxiety & тв & COPD & Oth NTD & ID \\
\hline Zimbabwe & Oth HIV & MDD & Iron & Back pain & Neck pain & TB & Anxiety & ID & COPD & Schisto \\
\hline Western sub-Saharan Africa & Iron & Back pain & MDD & Makria & Hearing & Neck pain & Schisto & COPD & Migraine & Oth HIV \\
\hline Benin & Iron & MDD & Back pain & Mabria & Schisto & Neck pain & Hearing & COPD & Migraine & Anxiety \\
\hline Burkina Faso & Iron & MDD & Malaria & Back pain & Neck pain & Hearing & COPD & Migraine & Anxiety & Diarrhoea \\
\hline Cameroon & MDD & Iron & Back pain & Oth HIV & Oncho & Mabria & Neck pain & Hearing & COPD & Migraine \\
\hline
\end{tabular}

Lancet. Author manuscript; available in PMC 2015 September 07. 
Page 58

\begin{tabular}{|c|c|c|c|c|c|c|c|c|c|c|}
\hline & 1 & 2 & 3 & 4 & 5 & 6 & 7 & 8 & 9 & 10 \\
\hline Cape Verde & MDD & Iron & Back pain & Neck pain & Hearing & COPD & Diabetes & \multirow{2}{*}{$\begin{array}{l}\text { Migraine } \\
\text { COPD }\end{array}$} & Anxiety & Other MSK \\
\hline Chad & Iron & Back pain & MDD & Neck pain & Diarrhoea & Hearing & \multirow{2}{*}{$\begin{array}{l}\text { Malaria } \\
\text { Oth HIV }\end{array}$} & & \multirow{2}{*}{$\begin{array}{l}\text { Migraine } \\
\text { COPD }\end{array}$} & Anxiety \\
\hline Côte d'lvoire & Iron & MDD & Back pain & Malaria & Neck pain & Hearing & & Schisto & & Migraine \\
\hline Ghana & MDD & Iron & Back pain & Neck pain & Malaria & COPD & Hearing & Migraine & Schisto & Anxiety \\
\hline Guinea & Iron & MDD & Back pain & Neck pain & Malaria & Hearing & \multirow{2}{*}{$\begin{array}{l}\text { COPD } \\
\text { COPD }\end{array}$} & Schisto & Migraine & Anxiety \\
\hline Guinea-Bissau & Iron & MDD & Back pain & Oth HIV & Neck pain & Hearing & & Migraine & Anxiety & \multirow{2}{*}{$\begin{array}{l}\text { Diarrhoea } \\
\text { War }\end{array}$} \\
\hline Liberia & Oncho & Iron & MDD & \multirow{2}{*}{$\begin{array}{l}\text { Back pain } \\
\text { Back pain }\end{array}$} & Malaria & Schisto & Neck pain & Hearing & COPD & \\
\hline Mali & Iron & MDD & Malaria & & Neck pain & Hearing & COPD & Migraine & Diarrhoea & Anxiety \\
\hline Mauritania & Iron & MDD & Back pain & Neck pain & Hearing & COPD & | Migraine & Refraction & Anxiety & Diarrhoea \\
\hline Niger & Iron & MDD & Back pain & Diarrhoea & Neck pain & Hearing & \multirow{2}{*}{$\begin{array}{l}\text { Malaria } \\
\text { Oth HIV }\end{array}$} & COPD & \multirow{2}{*}{$\begin{array}{l}\text { Migraine } \\
\text { COPD }\end{array}$} & Anxiety \\
\hline Nigeria & Back pain & Iron & MDD & Hearing & Malaria & Schisto & & \multirow{2}{*}{$\begin{array}{l}\text { Neck pain } \\
\text { Anxiety }\end{array}$} & & Migraine \\
\hline São Tomé and Principe & MDD & Iron & Back pain & Neck pain & Hearing & COPD & Migraine & & \multirow{2}{*}{$\begin{array}{l}\text { Diarrhoea } \\
\text { Diarrhoea }\end{array}$} & Other MSK \\
\hline Senegal & Iron & MDD & Back pain & Neck pain & Hearing & COPD & Diabetes & Migraine & & Anxiety \\
\hline Sierra Leone & Iron & MDD & Back pain & Neck pain & Malaria & Oncho & COPD & Migraine & Anxiety & Hearing \\
\hline The Gambia & MDD & Iron & Back pain & Neck pain & Hearing & Diarrhoea & Migraine & COPD & Anxiety & Schisto \\
\hline \multirow[t]{11}{*}{ Togo } & Iron & MDD & Back pain & Malaria & Neck pain & Diarrhoea & Hearing & Oth HIV & COPD & Migraine \\
\hline & \multicolumn{4}{|c|}{ Low back pain } & Back pain & & & & & \\
\hline & \multicolumn{4}{|c|}{ Major depressive disorder } & MDD & & & & & \\
\hline & \multicolumn{4}{|c|}{ Iron-deficiency anaemia } & Iron & & & & & \\
\hline & \multicolumn{4}{|l|}{ Neck pain } & Neck pain & & & & & \\
\hline & \multicolumn{4}{|c|}{ Age-related and other hearing loss } & Hearing & & & & & \\
\hline & \multicolumn{4}{|l|}{ Migraine } & Migraine & & & & & \\
\hline & \multicolumn{4}{|c|}{ Diabetes mellitus } & Diabetes & & & & & \\
\hline & \multicolumn{4}{|c|}{ Chronic obstructive pulmonary disease } & COPD & & & & & \\
\hline & \multicolumn{4}{|c|}{ Anxiety disorders } & Anxiety & & & & & \\
\hline & \multicolumn{4}{|c|}{ Other musculoskeletal disorders } & Other MSK & & & & & \\
\hline
\end{tabular}

Figure 4. Top ten causes of years lived with disability by location in 2013

Lancet. Author manuscript; available in PMC 2015 September 07. 

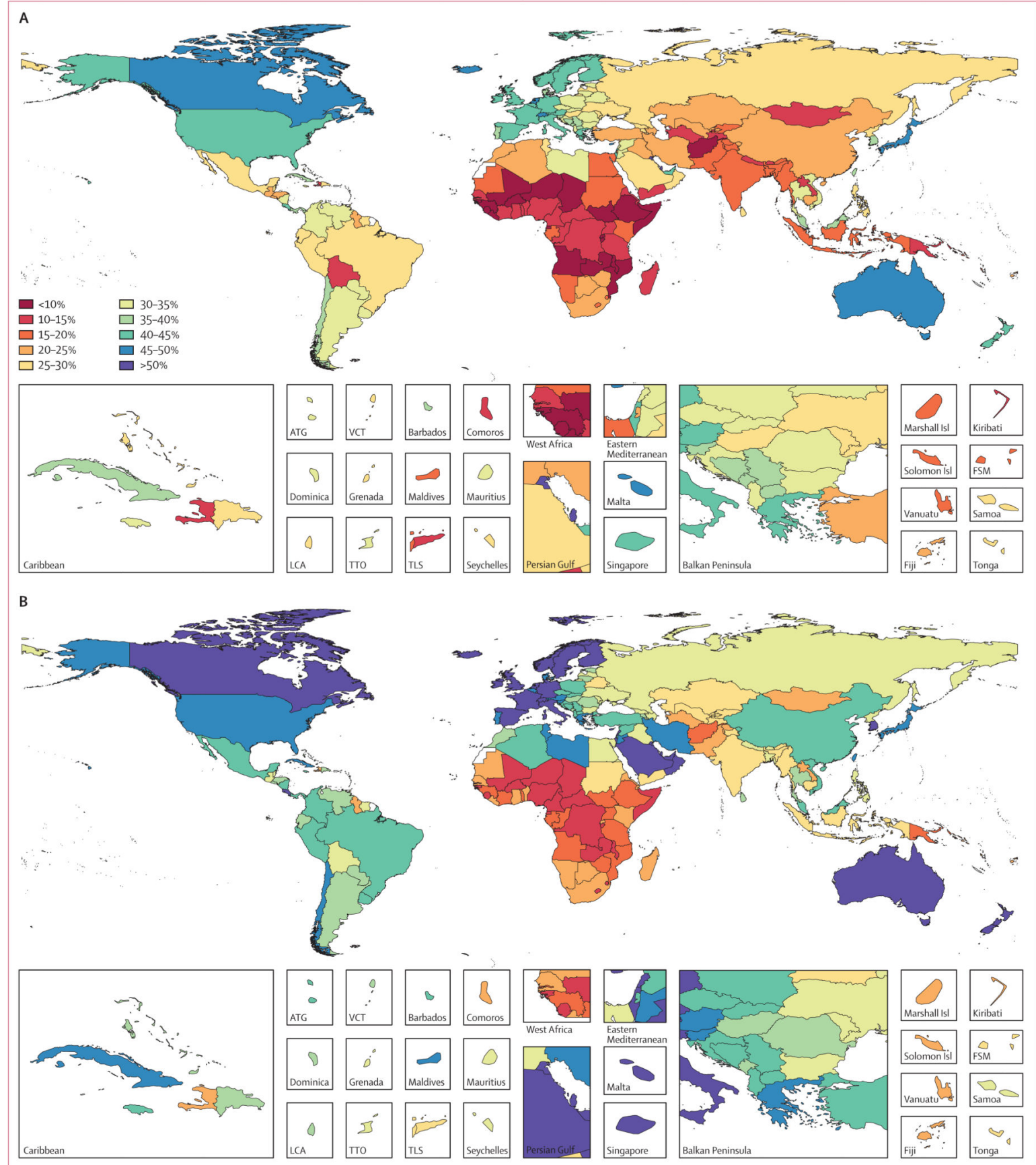

Figure 5. Percentage of disability-adjusted life years due to years lived with disability in 1990 (A) and $2013(B)$ in 188 countries

ATG=Antigua and Barbuda. FSM=Federated States of Micronesia. LCA=Saint Lucia.

TLS=Timor-Leste. TTO=Trinidad and Tobago. VCT=Saint Vincent and the Grenadines.

Isl=Islands. 
Table 1

GBD 2013 data representativeness index by cause

\begin{tabular}{|c|c|c|c|c|}
\hline & Before 1998 & 1998-2005 & 2006-13 & Total \\
\hline All causes & $100 \cdot 0 \%$ & $100 \cdot 0 \%$ & $100 \cdot 0 \%$ & $100 \cdot 0 \%$ \\
\hline Communicable, maternal, neonatal, and nutritional diseases & $99 \cdot 5 \%$ & $100 \cdot 0 \%$ & $100 \cdot 0 \%$ & $100 \cdot 0 \%$ \\
\hline HIV/AIDS and tuberculosis & $91 \cdot 0 \%$ & $96 \cdot 8 \%$ & $98 \cdot 9 \%$ & $99 \cdot 5 \%$ \\
\hline Tuberculosis & $56 \cdot 9 \%$ & $91 \cdot 5 \%$ & $98 \cdot 4 \%$ & $99 \cdot 5 \%$ \\
\hline HIV/AIDS & $79 \cdot 8 \%$ & $80 \cdot 3 \%$ & $79 \cdot 8 \%$ & $80 \cdot 3 \%$ \\
\hline HIV/AIDS resulting in mycobacterial infection & $3 \cdot 3 \%$ & $1 \cdot 1 \%$ & $0 \cdot 5 \%$ & $3 \cdot 3 \%$ \\
\hline HIV/AIDS resulting in other diseases & $79 \cdot 8 \%$ & $80 \cdot 3 \%$ & $79 \cdot 8 \%$ & $80 \cdot 3 \%$ \\
\hline Diarrhoea, lower respiratory, and other common infectious diseases & $97 \cdot 9 \%$ & $99 \cdot 5 \%$ & $100 \cdot 0 \%$ & $100 \cdot 0 \%$ \\
\hline Diarrhoeal diseases & $31 \cdot 9 \%$ & $58 \cdot 5 \%$ & $38 \cdot 3 \%$ & $67 \cdot 0 \%$ \\
\hline Intestinal infectious diseases & $18 \cdot 1 \%$ & $25 \cdot 0 \%$ & $10 \cdot 6 \%$ & $34 \cdot 6 \%$ \\
\hline Typhoid fever & $16 \cdot 5 \%$ & $10 \cdot 6 \%$ & $6 \cdot 4 \%$ & $20 \cdot 2 \%$ \\
\hline Paratyphoid fever & $4 \cdot 8 \%$ & $8.0 \%$ & $4 \cdot 8 \%$ & $9.0 \%$ \\
\hline Other intestinal infectious diseases & .. &.. & .. &.. \\
\hline Lower respiratory infections & $30 \cdot 9 \%$ & $61 \cdot 2 \%$ & $48.9 \%$ & $73 \cdot 4 \%$ \\
\hline Upper respiratory infections & $30 \cdot 9 \%$ & $28 \cdot 2 \%$ & $25 \cdot 0 \%$ & $44 \cdot 7 \%$ \\
\hline Otitis media & $18 \cdot 1 \%$ & $11 \cdot 7 \%$ & $5 \cdot 9 \%$ & $23 \cdot 4 \%$ \\
\hline Meningitis & $30 \cdot 3 \%$ & $36 \cdot 7 \%$ & $22 \cdot 3 \%$ & $47 \cdot 9 \%$ \\
\hline Pneumococcal meningitis & $26 \cdot 1 \%$ & $21 \cdot 3 \%$ & $8 \cdot 5 \%$ & $36 \cdot 7 \%$ \\
\hline Haemophilus influenzae type B meningitis & $26 \cdot 1 \%$ & $21 \cdot 8 \%$ & $8 \cdot 0 \%$ & $37 \cdot 2 \%$ \\
\hline Meningococcal meningitis & $25 \cdot 5 \%$ & $20 \cdot 2 \%$ & $8 \cdot 5 \%$ & $35 \cdot 6 \%$ \\
\hline Other meningitis & $25.5 \%$ & $20 \cdot 2 \%$ & $8.0 \%$ & $34.6 \%$ \\
\hline Encephalitis & $19 \cdot 7 \%$ & $20 \cdot 7 \%$ & $15 \cdot 4 \%$ & $30 \cdot 3 \%$ \\
\hline Diphtheria & $57 \cdot 4 \%$ & $61 \cdot 2 \%$ & $59 \cdot 6 \%$ & $71 \cdot 3 \%$ \\
\hline Whooping cough & $97 \cdot 3 \%$ & $96 \cdot 3 \%$ & $93 \cdot 6 \%$ & $98 \cdot 4 \%$ \\
\hline Tetanus & $64 \cdot 4 \%$ & $65 \cdot 4 \%$ & $61 \cdot 2 \%$ & $75 \cdot 0 \%$ \\
\hline Measles & $97 \cdot 3 \%$ & $97 \cdot 9 \%$ & $98.9 \%$ & $98 \cdot 9 \%$ \\
\hline Varicella and herpes zoster & $14 \cdot 4 \%$ & $17 \cdot 6 \%$ & $2 \cdot 7 \%$ & $21 \cdot 3 \%$ \\
\hline Neglected tropical diseases and malaria & $\mathbf{9 7 \cdot 3 \%}$ & $98.9 \%$ & $97 \cdot 9 \%$ & $99 \cdot 5 \%$ \\
\hline Malaria & $37 \cdot 8 \%$ & $31.9 \%$ & $23 \cdot 4 \%$ & $42 \cdot 6 \%$ \\
\hline Chagas disease & $42 \cdot 1 \%$ & $100 \cdot 0 \%$ & $57 \cdot 9 \%$ & $100 \cdot 0 \%$ \\
\hline Leishmaniasis & $41 \cdot 4 \%$ & $44 \cdot 1 \%$ & $44 \cdot 1 \%$ & $52 \cdot 6 \%$ \\
\hline Visceral leishmaniasis & $27 \cdot 6 \%$ & $32 \cdot 2 \%$ & $33 \cdot 6 \%$ & $40 \cdot 1 \%$ \\
\hline Cutaneous and mucocutaneous leishmaniasis & $29 \cdot 6 \%$ & $33 \cdot 6 \%$ & $34 \cdot 2 \%$ & $40 \cdot 8 \%$ \\
\hline African trypanosomiasis & $94.4 \%$ & $100 \cdot 0 \%$ & $100 \cdot 0 \%$ & $100 \cdot 0 \%$ \\
\hline Schistosomiasis & $50 \cdot 0 \%$ & $21 \cdot 2 \%$ & $4.5 \%$ & $50 \cdot 0 \%$ \\
\hline Cysticercosis & $4 \cdot 3 \%$ & $1.6 \%$ & $0 \cdot 5 \%$ & $6.4 \%$ \\
\hline
\end{tabular}




\begin{tabular}{|c|c|c|c|c|}
\hline & Before 1998 & 1998-2005 & 2006-13 & Total \\
\hline Cystic echinococcosis & $6.9 \%$ & $18 \cdot 1 \%$ & $14 \cdot 4 \%$ & $20 \cdot 2 \%$ \\
\hline Lymphatic filariasis & $37.9 \%$ & $37 \cdot 9 \%$ & $19.7 \%$ & $50 \cdot 0 \%$ \\
\hline Onchocerciasis & .. & .. & .. & .. \\
\hline Trachoma & $34.5 \%$ & $27.6 \%$ & $25.9 \%$ & $44.8 \%$ \\
\hline Dengue & $50 \cdot 4 \%$ & $54.8 \%$ & $54.8 \%$ & $60 \cdot 0 \%$ \\
\hline Yellow fever & $90 \cdot 9 \%$ & $95 \cdot 5 \%$ & $88.6 \%$ & $95 \cdot 5 \%$ \\
\hline Rabies & $49.5 \%$ & $61.2 \%$ & $59.6 \%$ & $67.6 \%$ \\
\hline Intestinal nematode infections & $92.7 \%$ & $88 \cdot 3 \%$ & $70 \cdot 1 \%$ & $99 \cdot 3 \%$ \\
\hline Ascariasis & $92 \cdot 7 \%$ & $88 \cdot 3 \%$ & $70 \cdot 1 \%$ & $99 \cdot 3 \%$ \\
\hline Trichuriasis & $92 \cdot 7 \%$ & $88.3 \%$ & $70 \cdot 1 \%$ & $99 \cdot 3 \%$ \\
\hline Hookworm disease & $92.0 \%$ & $88.3 \%$ & $69 \cdot 3 \%$ & $98.5 \%$ \\
\hline Food-borne trematodiases & $100 \cdot 0 \%$ & $35 \cdot 3 \%$ & $5.9 \%$ & $100 \cdot 0 \%$ \\
\hline Other neglected tropical diseases & .. & .. & .. & .. \\
\hline Maternal disorders & $33 \cdot 0 \%$ & $51 \cdot 6 \%$ & $47 \cdot 3 \%$ & $60 \cdot 6 \%$ \\
\hline Maternal haemorrhage & $10 \cdot 1 \%$ & $27.7 \%$ & $22.3 \%$ & $33.5 \%$ \\
\hline Maternal sepsis and other infections & $4.8 \%$ & $15 \cdot 4 \%$ & $15 \cdot 4 \%$ & $18.6 \%$ \\
\hline Maternal hypertensive disorders & $12 \cdot 8 \%$ & $34.0 \%$ & $36 \cdot 7 \%$ & $46 \cdot 8 \%$ \\
\hline Obstructed labour & $10 \cdot 1 \%$ & $23.4 \%$ & $23.9 \%$ & $29 \cdot 3 \%$ \\
\hline Complications of abortion & $3 \cdot 2 \%$ & $13 \cdot 3 \%$ & $13 \cdot 8 \%$ & $16 \cdot 0 \%$ \\
\hline Other maternal disorders & .. & .. & .. & .. \\
\hline Neonatal disorders & $68 \cdot 1 \%$ & $70 \cdot 2 \%$ & $\mathbf{5 8 \cdot 5 \%}$ & $81.9 \%$ \\
\hline Preterm birth complications & $36 \cdot 2 \%$ & $45 \cdot 2 \%$ & $31.4 \%$ & $55 \cdot 3 \%$ \\
\hline Neonatal encephalopathy due to birth asphyxia and trauma & $12 \cdot 2 \%$ & $14 \cdot 4 \%$ & $5.9 \%$ & $20 \cdot 2 \%$ \\
\hline Neonatal sepsis and other infections & $4.8 \%$ & $3 \cdot 2 \%$ & $0.5 \%$ & $6.9 \%$ \\
\hline Haemolytic disease and other neonatal jaundice & $42 \cdot 6 \%$ & $40 \cdot 4 \%$ & $35 \cdot 1 \%$ & $55 \cdot 3 \%$ \\
\hline Other neonatal disorders & .. & .. & .. & .. \\
\hline Nutritional deficiencies & $93 \cdot 6 \%$ & $95 \cdot 7 \%$ & $91 \cdot 0 \%$ & $98.9 \%$ \\
\hline Protein-energy malnutrition & $92.6 \%$ & $95 \cdot 7 \%$ & $91.0 \%$ & $98.9 \%$ \\
\hline Iodine deficiency & $37 \cdot 8 \%$ & $22 \cdot 3 \%$ & $2.7 \%$ & $45 \cdot 2 \%$ \\
\hline Vitamin A deficiency & $20 \cdot 8 \%$ & $5.0 \%$ & $0.8 \%$ & $22.5 \%$ \\
\hline Iron-deficiency anaemia & .. & .. & .. & .. \\
\hline Other nutritional deficiencies & .. & .. & .. & .. \\
\hline Other communicable, maternal, neonatal, and nutritional diseases & $82 \cdot 4 \%$ & $\mathbf{8 3 \cdot 0 \%}$ & $77 \cdot 1 \%$ & $92 \cdot 6 \%$ \\
\hline Sexually transmitted diseases excluding HIV & $37 \cdot 2 \%$ & $43 \cdot 1 \%$ & $19 \cdot 7 \%$ & $56 \cdot 4 \%$ \\
\hline Syphilis & $1.1 \%$ & $3.7 \%$ & $0.0 \%$ & $3.7 \%$ \\
\hline Chlamydial infection & $16 \cdot 5 \%$ & $26 \cdot 6 \%$ & $13.8 \%$ & $40 \cdot 4 \%$ \\
\hline Gonococcal infection & $15 \cdot 4 \%$ & $23.9 \%$ & $8.0 \%$ & $31.9 \%$ \\
\hline Trichomoniasis & $8.0 \%$ & $18 \cdot 1 \%$ & $9.6 \%$ & $26 \cdot 6 \%$ \\
\hline
\end{tabular}




\begin{tabular}{|c|c|c|c|c|}
\hline & Before 1998 & 1998-2005 & $2006-13$ & Total \\
\hline Genital herpes & $24.5 \%$ & $24.5 \%$ & $3.2 \%$ & $31.9 \%$ \\
\hline Other sexually transmitted diseases & $4.3 \%$ & $1.1 \%$ & $0.5 \%$ & $4.8 \%$ \\
\hline Hepatitis & $56 \cdot 9 \%$ & $45 \cdot 7 \%$ & $19.7 \%$ & $68 \cdot 1 \%$ \\
\hline Hepatitis A & $46 \cdot 8 \%$ & $27.7 \%$ & $12 \cdot 2 \%$ & $56 \cdot 4 \%$ \\
\hline Hepatitis B & $36 \cdot 2 \%$ & $27.7 \%$ & $5.3 \%$ & $43.6 \%$ \\
\hline Hepatitis C & $26 \cdot 6 \%$ & $28.2 \%$ & $8.5 \%$ & $38.3 \%$ \\
\hline Hepatitis E & $20 \cdot 7 \%$ & $12 \cdot 2 \%$ & $5.9 \%$ & $25.5 \%$ \\
\hline Leprosy & $88 \cdot 1 \%$ & $77.6 \%$ & $85 \cdot 3 \%$ & $99 \cdot 3 \%$ \\
\hline Other infectious diseases & $3.7 \%$ & $2 \cdot 1 \%$ & $0.0 \%$ & $4.3 \%$ \\
\hline Non-communicable diseases & $98 \cdot 4 \%$ & $99 \cdot 5 \%$ & $98 \cdot 4 \%$ & $99 \cdot 5 \%$ \\
\hline Neoplasms & $\mathbf{7 3 . 9 \%}$ & $74 \cdot 5 \%$ & $69 \cdot 1 \%$ & $82 \cdot 4 \%$ \\
\hline Oesophageal cancer & $65 \cdot 4 \%$ & $68 \cdot 1 \%$ & $61 \cdot 2 \%$ & $76 \cdot 1 \%$ \\
\hline Stomach cancer & $65 \cdot 4 \%$ & $69 \cdot 1 \%$ & $61.7 \%$ & $76 \cdot 6 \%$ \\
\hline Liver cancer & $66 \cdot 5 \%$ & $71 \cdot 3 \%$ & $63 \cdot 8 \%$ & $78.7 \%$ \\
\hline Liver cancer due to hepatitis B & $17 \cdot 0 \%$ & $17 \cdot 6 \%$ & $9.6 \%$ & $24.5 \%$ \\
\hline Liver cancer due to hepatitis $\mathrm{C}$ & $17 \cdot 6 \%$ & $17 \cdot 6 \%$ & $10 \cdot 1 \%$ & $24.5 \%$ \\
\hline Liver cancer due to alcohol use & $6.9 \%$ & $9.6 \%$ & $6.4 \%$ & $11 \cdot 2 \%$ \\
\hline Liver cancer due to other causes & $3.7 \%$ & $5.3 \%$ & $4.3 \%$ & $5.9 \%$ \\
\hline Larynx cancer & $65 \cdot 4 \%$ & $68 \cdot 1 \%$ & $61.2 \%$ & $75 \cdot 5 \%$ \\
\hline Tracheal, bronchus, and lung cancer & $65 \cdot 4 \%$ & $69 \cdot 1 \%$ & $61.7 \%$ & $76 \cdot 6 \%$ \\
\hline Breast cancer & $66 \cdot 0 \%$ & $69 \cdot 1 \%$ & $61.7 \%$ & $76 \cdot 6 \%$ \\
\hline Cervical cancer & $65.4 \%$ & $69 \cdot 1 \%$ & $61 \cdot 2 \%$ & $76 \cdot 6 \%$ \\
\hline Uterine cancer & $65 \cdot 4 \%$ & $68.6 \%$ & $61.7 \%$ & $76 \cdot 6 \%$ \\
\hline Prostate cancer & $65 \cdot 4 \%$ & $69 \cdot 1 \%$ & $61.7 \%$ & $76 \cdot 6 \%$ \\
\hline Colon and rectum cancer & $65 \cdot 4 \%$ & $69 \cdot 1 \%$ & $61 \cdot 2 \%$ & $76 \cdot 1 \%$ \\
\hline Lip and oral cavity cancer & $60 \cdot 1 \%$ & $66 \cdot 5 \%$ & $60 \cdot 1 \%$ & $75.0 \%$ \\
\hline Nasopharynx cancer & $60 \cdot 1 \%$ & $66 \cdot 0 \%$ & $60 \cdot 1 \%$ & $74.5 \%$ \\
\hline Other pharynx cancer & $60 \cdot 1 \%$ & $66 \cdot 0 \%$ & $60 \cdot 1 \%$ & $74.5 \%$ \\
\hline Gallbladder and biliary tract cancer & $60 \cdot 1 \%$ & $66 \cdot 0 \%$ & $60 \cdot 1 \%$ & $74.5 \%$ \\
\hline Pancreatic cancer & $60 \cdot 6 \%$ & $67.6 \%$ & $60 \cdot 6 \%$ & $75 \cdot 0 \%$ \\
\hline Malignant skin melanoma & $62 \cdot 2 \%$ & $67.0 \%$ & $60 \cdot 6 \%$ & $75 \cdot 0 \%$ \\
\hline Non-melanoma skin cancer & $60 \cdot 1 \%$ & $68 \cdot 1 \%$ & $62 \cdot 2 \%$ & $75.0 \%$ \\
\hline Ovarian cancer & $60 \cdot 6 \%$ & $66.5 \%$ & $61.2 \%$ & $74.5 \%$ \\
\hline Testicular cancer & $60 \cdot 6 \%$ & $66.5 \%$ & $60 \cdot 1 \%$ & $74.5 \%$ \\
\hline Kidney cancer & $60 \cdot 6 \%$ & $66 \cdot 5 \%$ & $60 \cdot 1 \%$ & $74.5 \%$ \\
\hline Bladder cancer & $60 \cdot 6 \%$ & $67.0 \%$ & $60 \cdot 6 \%$ & $74.5 \%$ \\
\hline Brain and nervous system cancer & $60 \cdot 6 \%$ & $67 \cdot 6 \%$ & $61.2 \%$ & $75 \cdot 0 \%$ \\
\hline Thyroid cancer & $60 \cdot 6 \%$ & $66 \cdot 0 \%$ & $60 \cdot 1 \%$ & $74.5 \%$ \\
\hline Mesothelioma & $53 \cdot 7 \%$ & $64 \cdot 4 \%$ & $58.0 \%$ & $73.9 \%$ \\
\hline Hodgkin's lymphoma & $60 \cdot 6 \%$ & $66 \cdot 5 \%$ & $60 \cdot 1 \%$ & $74.5 \%$ \\
\hline Non-Hodgkin lymphoma & $60 \cdot 6 \%$ & $67.6 \%$ & $60 \cdot 6 \%$ & $75.5 \%$ \\
\hline
\end{tabular}

Lancet. Author manuscript; available in PMC 2015 September 07. 


\begin{tabular}{|c|c|c|c|c|}
\hline & Before 1998 & 1998-2005 & $2006-13$ & Total \\
\hline Multiple myeloma & $60 \cdot 6 \%$ & $67.0 \%$ & $60 \cdot 6 \%$ & $75.0 \%$ \\
\hline Leukaemia & $66 \cdot 0 \%$ & $69 \cdot 1 \%$ & $62 \cdot 2 \%$ & $77 \cdot 1 \%$ \\
\hline Other neoplasms & $66 \cdot 0 \%$ & $69 \cdot 1 \%$ & $62 \cdot 8 \%$ & $77 \cdot 7 \%$ \\
\hline Cardiovascular diseases & $71 \cdot 3 \%$ & $76 \cdot 6 \%$ & $69 \cdot 1 \%$ & $86 \cdot 2 \%$ \\
\hline Rheumatic heart disease & $15 \cdot 4 \%$ & $19 \cdot 1 \%$ & $18.6 \%$ & $34.0 \%$ \\
\hline Ischaemic heart disease & $21.8 \%$ & $42.6 \%$ & $13 \cdot 8 \%$ & $50 \cdot 0 \%$ \\
\hline Cerebrovascular disease & $63 \cdot 8 \%$ & $64.9 \%$ & $63 \cdot 3 \%$ & $75.5 \%$ \\
\hline Ischaemic stroke & $59 \cdot 0 \%$ & $64 \cdot 4 \%$ & $62 \cdot 8 \%$ & $73.9 \%$ \\
\hline Haemorrhagic stroke & $59 \cdot 6 \%$ & $64.4 \%$ & $62.2 \%$ & $73.4 \%$ \\
\hline Hypertensive heart disease & $11.7 \%$ & $10 \cdot 1 \%$ & $8.5 \%$ & $18.6 \%$ \\
\hline Cardiomyopathy and myocarditis & $12 \cdot 8 \%$ & $22.9 \%$ & $19 \cdot 1 \%$ & $30 \cdot 9 \%$ \\
\hline Atrial fibrillation and flutter & $8.5 \%$ & $10 \cdot 6 \%$ & $5.9 \%$ & $13 \cdot 3 \%$ \\
\hline Peripheral vascular disease & $3.7 \%$ & $9.0 \%$ & $4.3 \%$ & $11.7 \%$ \\
\hline Endocarditis & $5.9 \%$ & $16 \cdot 0 \%$ & $14 \cdot 4 \%$ & $17 \cdot 6 \%$ \\
\hline Other cardiovascular and circulatory diseases & $0.0 \%$ & $0.5 \%$ & $0.5 \%$ & $0.5 \%$ \\
\hline Chronic respiratory diseases & $38 \cdot 3 \%$ & $64 \cdot 9 \%$ & $32 \cdot 4 \%$ & $68 \cdot 6 \%$ \\
\hline Chronic obstructive pulmonary disease & $11.7 \%$ & $17 \cdot 0 \%$ & $9.0 \%$ & $22 \cdot 3 \%$ \\
\hline Pneumoconiosis & $2.7 \%$ & $13 \cdot 3 \%$ & $13 \cdot 3 \%$ & $15 \cdot 4 \%$ \\
\hline Silicosis & $2.7 \%$ & $13 \cdot 3 \%$ & $13 \cdot 3 \%$ & $15 \cdot 4 \%$ \\
\hline Asbestosis & $11 \cdot 8 \%$ & $17 \cdot 6 \%$ & $17 \cdot 6 \%$ & $23.5 \%$ \\
\hline Coal workers' pneumoconiosis & $5.7 \%$ & $20 \cdot 0 \%$ & $20 \cdot 0 \%$ & $22.9 \%$ \\
\hline Other pneumoconiosis & $1.6 \%$ & $12 \cdot 2 \%$ & $11.7 \%$ & $13 \cdot 3 \%$ \\
\hline Asthma & $34 \cdot 6 \%$ & $64.9 \%$ & $25.0 \%$ & $68 \cdot 6$ \\
\hline Interstitial lung disease and pulmonary sarcoidosis & $8.0 \%$ & $16 \cdot 5 \%$ & $13 \cdot 8 \%$ & $18 \cdot 1 \%$ \\
\hline Other chronic respiratory diseases & .. &. & .. &.. \\
\hline Cirrhosis & $15 \cdot 4 \%$ & $19 \cdot 1 \%$ & $18 \cdot 1 \%$ & $29 \cdot 3 \%$ \\
\hline Cirrhosis due to hepatitis B & $10 \cdot 6 \%$ & $6.9 \%$ & $4.3 \%$ & $14.9 \%$ \\
\hline Cirrhosis due to hepatitis $\mathrm{C}$ & $12 \cdot 8 \%$ & $7.4 \%$ & $4.3 \%$ & $16 \cdot 5 \%$ \\
\hline Cirrhosis due to alcohol use & $6.9 \%$ & $5.3 \%$ & $3.7 \%$ & $9.6 \%$ \\
\hline Cirrhosis due to other causes & $5.3 \%$ & $5 \cdot 3 \%$ & $4.3 \%$ & $8.5 \%$ \\
\hline Digestive diseases & $19 \cdot 7 \%$ & $25 \cdot 0 \%$ & $18 \cdot 6 \%$ & $30 \cdot 3 \%$ \\
\hline Peptic ulcer disease & $5 \cdot 3 \%$ & $14 \cdot 4 \%$ & $14.9 \%$ & $17 \cdot 0 \%$ \\
\hline Gastritis and duodenitis & $2.7 \%$ & $13.8 \%$ & $13 \cdot 8 \%$ & $15 \cdot 4 \%$ \\
\hline Appendicitis & $2 \cdot 1 \%$ & $13 \cdot 3 \%$ & $13 \cdot 8 \%$ & $14.9 \%$ \\
\hline Paralytic ileus and intestinal obstruction & $3 \cdot 2 \%$ & $15 \cdot 4 \%$ & $13 \cdot 8 \%$ & $17 \cdot 0 \%$ \\
\hline Inguinal, femoral, and abdominal hernia & $2 \cdot 1 \%$ & $13 \cdot 3 \%$ & $13 \cdot 8 \%$ & $14.9 \%$ \\
\hline Inflammatory bowel disease & $12 \cdot 8 \%$ & $14.4 \%$ & $6.4 \%$ & $19 \cdot 1 \%$ \\
\hline Vascular intestinal disorders & $2.1 \%$ & $13 \cdot 3 \%$ & $13 \cdot 8 \%$ & $14.9 \%$ \\
\hline
\end{tabular}




\begin{tabular}{|c|c|c|c|c|}
\hline & Before 1998 & 1998-2005 & 2006-13 & Total \\
\hline Gallbladder and biliary diseases & $10 \cdot 6 \%$ & $16 \cdot 5 \%$ & $14.4 \%$ & $22.3 \%$ \\
\hline Pancreatitis & $4.8 \%$ & $14.9 \%$ & $14.9 \%$ & $16 \cdot 0 \%$ \\
\hline Other digestive diseases & .. &.. & .. & .. \\
\hline Neurological disorders & $46 \cdot 3 \%$ & $37 \cdot 2 \%$ & $28 \cdot 7 \%$ & $55 \cdot 3 \%$ \\
\hline Alzheimer's disease and other dementias & $18 \cdot 6 \%$ & $15 \cdot 4 \%$ & $12 \cdot 2 \%$ & $22.3 \%$ \\
\hline Parkinson's disease & $18 \cdot 1 \%$ & $14.9 \%$ & $7 \cdot 4 \%$ & $23.4 \%$ \\
\hline Epilepsy & $21 \cdot 3 \%$ & $16 \cdot 0 \%$ & $3.7 \%$ & $28.2 \%$ \\
\hline Multiple sclerosis & $21 \cdot 3 \%$ & $16 \cdot 0 \%$ & $7 \cdot 4 \%$ & $26 \cdot 1 \%$ \\
\hline Migraine & $16 \cdot 0 \%$ & $13.3 \%$ & $11.7 \%$ & $25 \cdot 0 \%$ \\
\hline Tension-type headache & $9.0 \%$ & $6.4 \%$ & $10 \cdot 6 \%$ & $19 \cdot 1 \%$ \\
\hline Medication overuse headache & $3 \cdot 2 \%$ & $4.8 \%$ & $9.0 \%$ & $11 \cdot 2 \%$ \\
\hline Other neurological disorders & $8.5 \%$ & $3.2 \%$ & $0.0 \%$ & $9.0 \%$ \\
\hline Mental and substance use disorders & $37 \cdot 8 \%$ & $\mathbf{5 8 . 5 \%}$ & $35 \cdot 6 \%$ & $67.6 \%$ \\
\hline Schizophrenia & $17 \cdot 0 \%$ & $9.0 \%$ & $3.7 \%$ & $19 \cdot 1 \%$ \\
\hline Alcohol use disorders & $19 \cdot 7 \%$ & $28.7 \%$ & $14.9 \%$ & $31.4 \%$ \\
\hline Drug use disorders & $20 \cdot 7 \%$ & $47 \cdot 3 \%$ & $26 \cdot 1 \%$ & $51 \cdot 6 \%$ \\
\hline Opioid use disorders & $12 \cdot 8 \%$ & $17 \cdot 6 \%$ & $2.7 \%$ & $19 \cdot 7 \%$ \\
\hline Cocaine use disorders & $6.9 \%$ & $31.9 \%$ & $5.9 \%$ & $34.6 \%$ \\
\hline Amphetamine use disorders & $6 \cdot 4 \%$ & $23.9 \%$ & $8.0 \%$ & $27.7 \%$ \\
\hline Cannabis use disorders & $16 \cdot 0 \%$ & $42.0 \%$ & $20 \cdot 7 \%$ & $46 \cdot 8 \%$ \\
\hline Other drug use disorders & .. & .. & .. & .. \\
\hline Depressive disorders & $19 \cdot 7 \%$ & $23.9 \%$ & $11.2 \%$ & $33.0 \%$ \\
\hline Major depressive disorder & $19 \cdot 7 \%$ & $23.9 \%$ & $11 \cdot 2 \%$ & $33.0 \%$ \\
\hline Dysthymia & $9.0 \%$ & $13 \cdot 8 \%$ & $5.3 \%$ & $18.6 \%$ \\
\hline Bipolar disorder & $8.5 \%$ & $16 \cdot 0 \%$ & $3.7 \%$ & $18.6 \%$ \\
\hline Anxiety disorders & $12 \cdot 8 \%$ & $21 \cdot 8 \%$ & $5.3 \%$ & $26 \cdot 1 \%$ \\
\hline Eating disorders & $10 \cdot 6 \%$ & $12 \cdot 2 \%$ & $4.3 \%$ & $14.9 \%$ \\
\hline Anorexia nervosa & $10 \cdot 1 \%$ & $12 \cdot 2 \%$ & $4.3 \%$ & $14.4 \%$ \\
\hline Bulimia nervosa & $8.5 \%$ & $11 \cdot 7 \%$ & $3.2 \%$ & $14.9 \%$ \\
\hline Autistic spectrum disorders & $5 \cdot 3 \%$ & $5.9 \%$ & $3.7 \%$ & $9.6 \%$ \\
\hline Autism & $5 \cdot 3 \%$ & $5 \cdot 3 \%$ & $3.7 \%$ & $9.6 \%$ \\
\hline Asperger's syndrome & $1.6 \%$ & $4.8 \%$ & $1.6 \%$ & $5.3 \%$ \\
\hline Attention-deficit or hyperactivity disorder & $10 \cdot 6 \%$ & $10 \cdot 1 \%$ & $4.8 \%$ & $19 \cdot 1 \%$ \\
\hline Conduct disorder & $5 \cdot 9 \%$ & $6.4 \%$ & $1.6 \%$ & $11 \cdot 2 \%$ \\
\hline Idiopathic intellectual disability & $6.4 \%$ & $3.2 \%$ & $1.1 \%$ & $7 \cdot 4 \%$ \\
\hline Other mental and substance use disorders & $0.5 \%$ & $0.5 \%$ & $0.0 \%$ & $1 \cdot 1 \%$ \\
\hline Diabetes, urogenital, blood, and endocrine diseases & $\mathbf{9 7 . 9 \%}$ & $98 \cdot 4 \%$ & $92 \cdot 0 \%$ & $98 \cdot 4 \%$ \\
\hline Diabetes mellitus & $36 \cdot 7 \%$ & $35 \cdot 1 \%$ & $33 \cdot 5 \%$ & $58.5 \%$ \\
\hline Acute glomerulonephritis & $5 \cdot 3 \%$ & $18 \cdot 1 \%$ & $5.9 \%$ & $19 \cdot 1 \%$ \\
\hline
\end{tabular}




\begin{tabular}{|c|c|c|c|c|}
\hline & Before 1998 & 1998-2005 & 2006-13 & Total \\
\hline Chronic kidney disease & $79 \cdot 8 \%$ & $82 \cdot 4 \%$ & $75 \cdot 5 \%$ & $89.4 \%$ \\
\hline Chronic kidney disease due to diabetes mellitus & $6.9 \%$ & $8.5 \%$ & $19 \cdot 1 \%$ & $27 \cdot 1 \%$ \\
\hline Chronic kidney disease due to hypertension & $6.9 \%$ & $11.7 \%$ & $19 \cdot 1 \%$ & $28.7 \%$ \\
\hline Chronic kidney disease due to glomerulonephritis & $5.9 \%$ & $7.4 \%$ & $19 \cdot 1 \%$ & $26 \cdot 1 \%$ \\
\hline Chronic kidney disease due to other causes & $3 \cdot 2 \%$ & $5.9 \%$ & $19 \cdot 1 \%$ & $24.5 \%$ \\
\hline Urinary diseases and male infertility & $15 \cdot 4 \%$ & $21 \cdot 3 \%$ & $17 \cdot 6 \%$ & $28.7 \%$ \\
\hline Interstitial nephritis and urinary tract infections & $2 \cdot 1 \%$ & $13 \cdot 3 \%$ & $13.8 \%$ & $14.9 \%$ \\
\hline Urolithiasis & $8.5 \%$ & $18 \cdot 1 \%$ & $16 \cdot 0 \%$ & $20.2 \%$ \\
\hline Benign prostatic hyperplasia & $6.9 \%$ & $16 \cdot 0 \%$ & $14 \cdot 4 \%$ & $19.7 \%$ \\
\hline Male infertility due to other causes & $5 \cdot 3 \%$ & $3.2 \%$ & $1.6 \%$ & $8.0 \%$ \\
\hline Other urinary diseases & .. & .. & .. & .. \\
\hline Gynaecological diseases & $66 \cdot 0 \%$ & $95 \cdot 2 \%$ & $22.9 \%$ & $95.2 \%$ \\
\hline Uterine fibroids & $2 \cdot 1 \%$ & $3.2 \%$ & $4.8 \%$ & $6.9 \%$ \\
\hline Polycystic ovarian syndrome & $2 \cdot 1 \%$ & $4.8 \%$ & $3 \cdot 2 \%$ & $8.0 \%$ \\
\hline Female infertility due to other causes & $5 \cdot 3 \%$ & $3.2 \%$ & $1.6 \%$ & $8.0 \%$ \\
\hline Endometriosis & $3.7 \%$ & $3.7 \%$ & $4.8 \%$ & $6.4 \%$ \\
\hline Genital prolapse & $3.2 \%$ & $3.7 \%$ & $0.5 \%$ & $8.0 \%$ \\
\hline Premenstrual syndrome & $64.4 \%$ & $95 \cdot 2 \%$ & $6.9 \%$ & $95.2 \%$ \\
\hline Other gynaecological diseases & $1.6 \%$ & $13 \cdot 3 \%$ & $13 \cdot 8 \%$ & $14.9 \%$ \\
\hline Haemoglobinopathies and haemolytic anaemias & $97.9 \%$ & $97.9 \%$ & $60 \cdot 1 \%$ & $97.9 \%$ \\
\hline Thalassaemias & $87.2 \%$ & $87.2 \%$ & $38.8 \%$ & $87.2 \%$ \\
\hline Thalassaemia trait & $1 \cdot 1 \%$ & $0.0 \%$ & $0.0 \%$ & $1 \cdot 1 \%$ \\
\hline Sickle cell disorders & $96 \cdot 3 \%$ & $96 \cdot 8 \%$ & $52 \cdot 1 \%$ & $96.8 \%$ \\
\hline Sickle cell trait & $0.5 \%$ & $0.0 \%$ & $0.0 \%$ & $0.5 \%$ \\
\hline Glucose-6-phosphate dehydrogenase deficiency & $39.9 \%$ & $24 \cdot 5 \%$ & $5.3 \%$ & $46 \cdot 3 \%$ \\
\hline Glucose-6-phosphate dehydrogenase deficiency trait & $0.5 \%$ & $0.5 \%$ & $0.5 \%$ & $1.6 \%$ \\
\hline Other haemoglobinopathies and haemolytic anaemias & .. & .. & .. & .. \\
\hline Endocrine, metabolic, blood, and immune disorders & $1.6 \%$ & $13 \cdot 3 \%$ & $13 \cdot 8 \%$ & $14.9 \%$ \\
\hline Musculoskeletal disorders & $22.9 \%$ & $44 \cdot 1 \%$ & $20 \cdot 2 \%$ & $51 \cdot 1 \%$ \\
\hline Rheumatoid arthritis & $16 \cdot 5 \%$ & $13 \cdot 8 \%$ & $9.6 \%$ & $24.5 \%$ \\
\hline Osteoarthritis & $10 \cdot 6 \%$ & $10 \cdot 1 \%$ & $7 \cdot 4 \%$ & $18 \cdot 1 \%$ \\
\hline Low back and neck pain & $12 \cdot 8 \%$ & $41.5 \%$ & $13 \cdot 3 \%$ & $46.3 \%$ \\
\hline Low back pain & $12 \cdot 8 \%$ & $40 \cdot 4 \%$ & $12 \cdot 2 \%$ & $46.3 \%$ \\
\hline Neck pain & $6 \cdot 4 \%$ & $12 \cdot 8 \%$ & $3 \cdot 2 \%$ & $15.4 \%$ \\
\hline Gout & $11 \cdot 2 \%$ & $9.6 \%$ & $4.8 \%$ & $18.1 \%$ \\
\hline Other musculoskeletal disorders & $6 \cdot 4 \%$ & $5.9 \%$ & $2 \cdot 1 \%$ & $10 \cdot 1 \%$ \\
\hline Other non-communicable diseases & $\mathbf{5 8 . 5 \%}$ & $61 \cdot 2 \%$ & $47.9 \%$ & $75.0 \%$ \\
\hline Congenital anomalies & $26.6 \%$ & $28 \cdot 7 \%$ & $21.3 \%$ & $32.4 \%$ \\
\hline Neural tube defects & $14 \cdot 4 \%$ & $20 \cdot 2 \%$ & $20 \cdot 7 \%$ & $22.3 \%$ \\
\hline Congenital heart anomalies & $20 \cdot 7 \%$ & $22.9 \%$ & $20 \cdot 7 \%$ & $25.5 \%$ \\
\hline
\end{tabular}




\begin{tabular}{|c|c|c|c|c|}
\hline & Before 1998 & 1998-2005 & 2006-13 & Total \\
\hline Orofacial clefts & $21 \cdot 8 \%$ & $24 \cdot 5 \%$ & $20 \cdot 7 \%$ & $25 \cdot 5 \%$ \\
\hline Down's syndrome & $21 \cdot 8 \%$ & $23.9 \%$ & $20 \cdot 7 \%$ & $25 \cdot 5 \%$ \\
\hline Turner's syndrome & $8.0 \%$ & $10 \cdot 1 \%$ & $10 \cdot 1 \%$ & $10 \cdot 6 \%$ \\
\hline Klinefelter's syndrome & $8.5 \%$ & $10 \cdot 6 \%$ & $10 \cdot 6 \%$ & $11 \cdot 2 \%$ \\
\hline Chromosomal unbalanced rearrangements & $20 \cdot 7 \%$ & $23 \cdot 4 \%$ & $20 \cdot 7 \%$ & $25 \cdot 5 \%$ \\
\hline Other congenital anomalies & $11 \cdot 2 \%$ & $9.6 \%$ & $1.6 \%$ & $16 \cdot 5 \%$ \\
\hline Skin and subcutaneous diseases & $35 \cdot 1 \%$ & $46 \cdot 8 \%$ & $30 \cdot 9 \%$ & $51 \cdot 1 \%$ \\
\hline Dermatitis & $32 \cdot 4 \%$ & $39.9 \%$ & $20 \cdot 2 \%$ & $43 \cdot 6 \%$ \\
\hline Psoriasis & $4.8 \%$ & $9.0 \%$ & $4.8 \%$ & $13 \cdot 8 \%$ \\
\hline Cellulitis & $1.6 \%$ & $12 \cdot 8 \%$ & $13 \cdot 3 \%$ & $14.4 \%$ \\
\hline Bacterial skin diseases & $3.7 \%$ & $18 \cdot 6 \%$ & $15 \cdot 4 \%$ & $21 \cdot 8 \%$ \\
\hline Scabies & $8.5 \%$ & $9.0 \%$ & $5.9 \%$ & $16 \cdot 5 \%$ \\
\hline Fungal skin diseases & $2.7 \%$ & $8.5 \%$ & $5.9 \%$ & $12 \cdot 2 \%$ \\
\hline Viral skin diseases & $6 \cdot 4 \%$ & $7 \cdot 4 \%$ & $5.3 \%$ & $12 \cdot 8 \%$ \\
\hline Acne vulgaris & $5.9 \%$ & $6.9 \%$ & $5.9 \%$ & $13 \cdot 8 \%$ \\
\hline Alopecia areata & $2.1 \%$ & $2.7 \%$ & $2.1 \%$ & $4.8 \%$ \\
\hline Pruritus & $2.1 \%$ & $3.7 \%$ & $3.2 \%$ & $6.9 \%$ \\
\hline Urticaria & $3 \cdot 2 \%$ & $4.3 \%$ & $3.2 \%$ & $8.5 \%$ \\
\hline Decubitus ulcer & $2.1 \%$ & $12.8 \%$ & $13 \cdot 3 \%$ & $14.4 \%$ \\
\hline Other skin and subcutaneous diseases & $0.5 \%$ & $0.5 \%$ & $0.5 \%$ & $0.5 \%$ \\
\hline Sense organ diseases & $22 \cdot 3 \%$ & $27 \cdot 7 \%$ & $14 \cdot 4 \%$ & $45 \cdot 7 \%$ \\
\hline Glaucoma & $14.9 \%$ & $10 \cdot 6 \%$ & $6.9 \%$ & $23.4 \%$ \\
\hline Cataract & $17 \cdot 0 \%$ & $19 \cdot 1 \%$ & $11.7 \%$ & $36 \cdot 7 \%$ \\
\hline Macular degeneration & $12 \cdot 2 \%$ & $12 \cdot 2 \%$ & $6 \cdot 9 \%$ & $23.9 \%$ \\
\hline Uncorrected refractive error & $0.0 \%$ & $0.0 \%$ & $3.2 \%$ & $3 \cdot 2 \%$ \\
\hline Age-related and other hearing loss & $12 \cdot 2 \%$ & $11 \cdot 2 \%$ & $2 \cdot 1 \%$ & $18 \cdot 1 \%$ \\
\hline Other vision loss & $8.5 \%$ & $7 \cdot 4 \%$ & $3.7 \%$ & $16 \cdot 0 \%$ \\
\hline Other sense organ diseases & $0.5 \%$ & $0.5 \%$ & $0.5 \%$ & $0.5 \%$ \\
\hline Oral disorders & $39 \cdot 4 \%$ & $30 \cdot 9 \%$ & $25 \cdot 0 \%$ & $51 \cdot 1 \%$ \\
\hline Deciduous caries & $28.7 \%$ & $21 \cdot 3 \%$ & $17 \cdot 6 \%$ & $42.6 \%$ \\
\hline Permanent caries & $29 \cdot 3 \%$ & $21 \cdot 3 \%$ & $16 \cdot 0 \%$ & $41 \cdot 0 \%$ \\
\hline Periodontal diseases & $18 \cdot 6 \%$ & $12 \cdot 8 \%$ & $5.3 \%$ & $25 \cdot 5 \%$ \\
\hline Edentulism and severe tooth loss & $9.6 \%$ & $9.0 \%$ & $9.0 \%$ & $16 \cdot 0 \%$ \\
\hline Other oral disorders & $0.5 \%$ & $0.5 \%$ & $0.5 \%$ & $0.5 \%$ \\
\hline Injuries & $\mathbf{9 4 \cdot 7 \%}$ & $\mathbf{9 4 \cdot 7 \%}$ & $\mathbf{9 4 \cdot 7 \%}$ & $96 \cdot 3 \%$ \\
\hline Transport injuries & $2 \cdot 7 \%$ & $34 \cdot 0 \%$ & $17 \cdot 0 \%$ & $42 \cdot 6 \%$ \\
\hline Road injuries & $2.7 \%$ & $34.0 \%$ & $16 \cdot 5 \%$ & $42.0 \%$ \\
\hline
\end{tabular}




\begin{tabular}{|c|c|c|c|c|}
\hline & Before 1998 & 1998-2005 & 2006-13 & Total \\
\hline Pedestrian road injuries & $1 \cdot 1 \%$ & $4.3 \%$ & $8.0 \%$ & $8.0 \%$ \\
\hline Cyclist road injuries & $1 \cdot 1 \%$ & $4.8 \%$ & $7 \cdot 4 \%$ & $8.0 \%$ \\
\hline Motorcyclist road injuries & $1 \cdot 1 \%$ & $4.3 \%$ & $7 \cdot 4 \%$ & $7 \cdot 4 \%$ \\
\hline Motor vehicle road injuries & $1 \cdot 1 \%$ & $4.8 \%$ & $7 \cdot 4 \%$ & $8.0 \%$ \\
\hline Other road injuries & $1 \cdot 1 \%$ & $4.3 \%$ & $7 \cdot 4 \%$ & $7 \cdot 4 \%$ \\
\hline Other transport injuries & $1 \cdot 1 \%$ & $4.3 \%$ & $8.5 \%$ & $8.5 \%$ \\
\hline Unintentional injuries & $3 \cdot 2 \%$ & $12 \cdot 2 \%$ & $13 \cdot 8 \%$ & $20 \cdot 2 \%$ \\
\hline Falls & $2 \cdot 1 \%$ & $10 \cdot 6 \%$ & $13 \cdot 3 \%$ & $18 \cdot 1 \%$ \\
\hline Drowning & $1 \cdot 1 \%$ & $7 \cdot 4 \%$ & $9.6 \%$ & $11.7 \%$ \\
\hline Fire, heat, and hot substances & $2.7 \%$ & $9.0 \%$ & $12.2 \%$ & $16 \cdot 0 \%$ \\
\hline Poisonings & $1.6 \%$ & $8.5 \%$ & $11.2 \%$ & $14.9 \%$ \\
\hline Exposure to mechanical forces & $1.6 \%$ & $8.5 \%$ & $9.6 \%$ & $13 \cdot 8 \%$ \\
\hline Unintentional firearm injuries & $1 \cdot 1 \%$ & $4.8 \%$ & $8.5 \%$ & $8 \cdot 5 \%$ \\
\hline Unintentional suffocation & $0.5 \%$ & $4.8 \%$ & $8.5 \%$ & $8.5 \%$ \\
\hline Other exposure to mechanical forces & $1 \cdot 1 \%$ & $4.8 \%$ & $8.5 \%$ & $8.5 \%$ \\
\hline Adverse effects of medical treatment & $1 \cdot 1 \%$ & $4.8 \%$ & $8.5 \%$ & $8.5 \%$ \\
\hline Animal contact & $2 \cdot 1 \%$ & $8.5 \%$ & $11.7 \%$ & $15 \cdot 4 \%$ \\
\hline Venomous animal contact & $1 \cdot 1 \%$ & $4.8 \%$ & $8.5 \%$ & $8.5 \%$ \\
\hline Non-venomous animal contact & $1 \cdot 1 \%$ & $4.8 \%$ & $8.5 \%$ & $8 \cdot 5 \%$ \\
\hline Foreign body & $1 \cdot 1 \%$ & $4.8 \%$ & $8.5 \%$ & $8.5 \%$ \\
\hline Foreign body in pulmonary aspiration and foreign body in airway & $1 \cdot 1 \%$ & $4.8 \%$ & $8.5 \%$ & $8.5 \%$ \\
\hline Foreign body in eyes & $1 \cdot 1 \%$ & $2.7 \%$ & $4.8 \%$ & $4.8 \%$ \\
\hline Foreign body in other body part & $1 \cdot 1 \%$ & $4.8 \%$ & $8.5 \%$ & $8.5 \%$ \\
\hline Other unintentional injuries & $2.7 \%$ & $10 \cdot 6 \%$ & $11.7 \%$ & $18 \cdot 1 \%$ \\
\hline Self-harm and interpersonal violence & $2 \cdot 1 \%$ & $9 \cdot 0 \%$ & $13 \cdot 3 \%$ & $16.5 \%$ \\
\hline Self-harm & $1 \cdot 1 \%$ & $7 \cdot 4 \%$ & $11.7 \%$ & $12 \cdot 8 \%$ \\
\hline Interpersonal violence & $2 \cdot 1 \%$ & $8.5 \%$ & $12 \cdot 2 \%$ & $16 \cdot 0 \%$ \\
\hline Assault by firearm & $1 \cdot 1 \%$ & $4.8 \%$ & $8.5 \%$ & $8.5 \%$ \\
\hline Assault by sharp object & $1 \cdot 1 \%$ & $4.8 \%$ & $8.5 \%$ & $8.5 \%$ \\
\hline Assault by other means & $1 \cdot 1 \%$ & $2 \cdot 1 \%$ & $3.7 \%$ & $3.7 \%$ \\
\hline Forces of nature, war, and legal intervention & $100 \cdot 0 \%$ & $\mathbf{9 8 \cdot 3 \%}$ & $\mathbf{9 8 . 9 \%}$ & $100 \cdot 0 \%$ \\
\hline Exposure to forces of nature & $100 \cdot 0 \%$ & $98 \cdot 8 \%$ & $99.4 \%$ & $100 \cdot 0 \%$ \\
\hline Collective violence and legal intervention & $100 \cdot 0 \%$ & $99 \cdot 1 \%$ & $99.1 \%$ & $100 \cdot 0 \%$ \\
\hline
\end{tabular}

GBD 2013=Global Burden of Diseases 2013 Study. 
Table 2

GBD 2013 data representativeness index by impairment, calculated as fraction of countries with data for each impairment and period

\begin{tabular}{|lrrrr|}
\hline & Before 1998 & $\mathbf{1 9 9 8}-\mathbf{2 0 0 5}$ & $\mathbf{2 0 0 6 - 1 3}$ & Total \\
\hline Anaemia & $61.2 \%$ & $56.9 \%$ & $22.3 \%$ & $74.5 \%$ \\
\hline Epilepsy & $35.6 \%$ & $23.4 \%$ & $11.7 \%$ & $44.1 \%$ \\
\hline Guillain-Barré syndrome & $12.2 \%$ & $5.9 \%$ & $0.0 \%$ & $13.3 \%$ \\
\hline Hearing loss & $10 \cdot 1 \%$ & $15.4 \%$ & $5.9 \%$ & $23.4 \%$ \\
\hline Heart failure & $10.1 \%$ & $17.6 \%$ & $15.4 \%$ & $21.3 \%$ \\
\hline Infertility & $49.5 \%$ & $34.6 \%$ & $27.1 \%$ & $60.1 \%$ \\
\hline Intellectual disability & $12.2 \%$ & $6.9 \%$ & $3.2 \%$ & $16.0 \%$ \\
\hline Pelvic inflammatory disease & $4.3 \%$ & $12.2 \%$ & $12.8 \%$ & $14.9 \%$ \\
\hline Vision loss & $22.3 \%$ & $46.8 \%$ & $24.5 \%$ & $60.1 \%$ \\
\hline
\end{tabular}

GBD 2013=Global Burden of Diseases 2013 Study. 
Table 3

Global incidence of acute sequelae (for less than 3 months) by cause for incidence greater than 1 million cases per year

\begin{tabular}{|c|c|c|c|c|c|c|}
\hline & $\begin{array}{l}\text { Cases } \\
\text { in } 1990 \\
(\times \\
1000)\end{array}$ & $\begin{array}{l}\text { Cases } \\
\text { in } 2013 \\
(\times \\
1000)\end{array}$ & Percentage change & $\begin{array}{l}\text { Age- } \\
\text { standardised } \\
\text { rate in } 1990 \\
\text { (per } 100000 \text { ) }\end{array}$ & $\begin{array}{l}\text { Age- } \\
\text { standardised } \\
\text { rate in } 2013 \\
\text { (per } 100000 \text { ) }\end{array}$ & Percentage change \\
\hline Upper respiratory infections & $\begin{array}{l}13557 \\
038(13 \\
317034 \\
\text { to } 13 \\
806 \\
346)\end{array}$ & $\begin{array}{l}18770 \\
589(18 \\
479508 \\
\text { to } 19 \\
048 \\
703)\end{array}$ & $\begin{array}{l}38 \cdot 26^{*}(35 \cdot 33 \text { to } \\
41 \cdot 60)\end{array}$ & $\begin{array}{l}243621 \cdot 2(239 \\
383 \cdot 6 \text { to } 248 \\
019 \cdot 3)\end{array}$ & $\begin{array}{l}259491 \cdot 0(255 \\
547 \cdot 1 \text { to } 263 \\
318 \cdot 4)\end{array}$ & $6 \cdot 48 *(4 \cdot 20$ to 8.95$)$ \\
\hline Diarrhoeal disease episodes & $\begin{array}{l}2920 \\
208(2 \\
866614 \\
\text { to } 2968 \\
429)\end{array}$ & $\begin{array}{l}2711 \\
253(2 \\
666452 \\
\text { to } 2761 \\
161)\end{array}$ & $\begin{array}{l}-7.29 * \\
-4.91)\end{array}$ & $\begin{array}{l}46265 \cdot 7(45 \\
440 \cdot 7 \text { to } 47 \\
003 \cdot 5)\end{array}$ & $\begin{array}{l}37467 \cdot 6(36 \\
858 \cdot 2 \text { to } 38 \\
151 \cdot 9)\end{array}$ & $\begin{array}{l}-19 \cdot 07^{*}(-20 \cdot 98 \text { to } \\
-17 \cdot 09)\end{array}$ \\
\hline $\begin{array}{l}\text { Other exposure to mechanical } \\
\text { forces }\end{array}$ & $\begin{array}{l}349533 \\
(334 \\
775 \text { to } \\
367 \\
702)\end{array}$ & $\begin{array}{l}381968 \\
(364 \\
953 \text { to } \\
401 \\
105)\end{array}$ & $9.28^{*}(6.86$ to 11.52$)$ & $\begin{array}{l}6049 \cdot 4(5797 \cdot 0 \\
\text { to } 6369 \cdot 6)\end{array}$ & $\begin{array}{l}5092 \cdot 8(4866 \cdot 9 \\
\text { to } 5355 \cdot 1)\end{array}$ & $\begin{array}{l}-15 \cdot 81^{*}(-17 \cdot 57 \text { to } \\
-14 \cdot 16)\end{array}$ \\
\hline Acute otitis media & $\begin{array}{l}339485 \\
(332 \\
992 \text { to } \\
345 \\
806)\end{array}$ & $\begin{array}{l}324720 \\
(318 \\
445 \text { to } \\
330 \\
958)\end{array}$ & $\begin{array}{l}-4.44^{*}(-7.03 \text { to } \\
-1.88)\end{array}$ & $\begin{array}{l}5292 \cdot 2(5194 \cdot 9 \\
\text { to } 5384 \cdot 2)\end{array}$ & $\begin{array}{l}4480 \cdot 9(4394 \cdot 0 \\
\text { to } 4566 \cdot 7)\end{array}$ & $\begin{array}{l}-15 \cdot 34^{*}(-17 \cdot 58 \text { to } \\
-13 \cdot 09)\end{array}$ \\
\hline $\begin{array}{l}\text { Tooth pain due to permanent } \\
\text { caries }\end{array}$ & $\begin{array}{l}164255 \\
(144 \\
960 \text { to } \\
184 \\
155)\end{array}$ & $\begin{array}{l}222966 \\
(194 \\
054 \text { to } \\
252 \\
697)\end{array}$ & $\begin{array}{l}35 \cdot 63^{*}(32.63 \text { to } \\
38 \cdot 69)\end{array}$ & $\begin{array}{l}3028 \cdot 4(2676 \cdot 9 \\
\text { to } 3391 \cdot 5)\end{array}$ & $\begin{array}{l}3070 \cdot 6(2672 \cdot 3 \\
\text { to } 3479 \cdot 0)\end{array}$ & $1.41(-0.94$ to 3.58$)$ \\
\hline Bacterial skin diseases & $\begin{array}{l}148035 \\
(123 \\
990 \text { to } \\
172 \\
137)\end{array}$ & $\begin{array}{l}154851 \\
(132 \\
130 \text { to } \\
180 \\
387)\end{array}$ & $4 \cdot 81(-4 \cdot 86$ to $14 \cdot 19)$ & $\begin{array}{l}2655 \cdot 7(2244 \cdot 7 \\
\text { to } 3075 \cdot 9)\end{array}$ & $\begin{array}{l}2194 \cdot 3(1870 \cdot 5 \\
\text { to } 2572 \cdot 9)\end{array}$ & $\begin{array}{l}-17 \cdot 21^{*}(-22 \cdot 61 \text { to } \\
-11.95)\end{array}$ \\
\hline Falls & $\begin{array}{l}107951 \\
(106 \\
004 \text { to } \\
109 \\
801)\end{array}$ & $\begin{array}{l}154533 \\
(151 \\
535 \text { to } \\
157 \\
392)\end{array}$ & $\begin{array}{l}43 \cdot 16^{*} \text { (39.53 to } \\
46 \cdot 97)\end{array}$ & $\begin{array}{l}2030 \cdot 3(1993 \cdot 1 \\
\text { to } 2063 \cdot 9)\end{array}$ & $\begin{array}{l}2017 \cdot 5(1988 \cdot 0 \\
\text { to } 2048 \cdot 0)\end{array}$ & $-0.62(-2.90$ to 1.69$)$ \\
\hline Lower respiratory infections & $\begin{array}{l}164622 \\
(162 \\
190 \text { to } \\
167 \\
306)\end{array}$ & $\begin{array}{l}150087 \\
(146 \\
724 \text { to } \\
152 \\
859)\end{array}$ & $\begin{array}{l}-8 \cdot 85^{*}(-12 \cdot 07 \text { to } \\
-6 \cdot 67)\end{array}$ & $\begin{array}{l}2891 \cdot 4(2849 \cdot 7 \\
\text { to } 2940 \cdot 9)\end{array}$ & $\begin{array}{l}2206 \cdot 9(2156 \cdot 5 \\
\text { to } 2246 \cdot 2)\end{array}$ & $\begin{array}{l}-23 \cdot 58 *(-26 \cdot 17 \text { to } \\
-21 \cdot 86)\end{array}$ \\
\hline Clinical episodes of malaria & $\begin{array}{l}172741 \\
(107 \\
735 \text { to } \\
279 \\
197)\end{array}$ & $\begin{array}{l}146761 \\
(85673 \\
\text { to } 249 \\
239)\end{array}$ & $\begin{array}{l}-16 \cdot 56(-34 \cdot 66 \text { to } \\
12 \cdot 48)\end{array}$ & $\begin{array}{l}2853 \cdot 7(1741 \cdot 1 \\
\text { to } 4755 \cdot 7)\end{array}$ & $\begin{array}{l}2036 \cdot 0(1184 \cdot 4 \\
\text { to } 3465 \cdot 6)\end{array}$ & $\begin{array}{l}-29 \cdot 81^{*}(-44.89 \text { to } \\
-5 \cdot 93)\end{array}$ \\
\hline Chlamydia infection & $\begin{array}{l}111204 \\
(108 \\
362 \text { to } \\
114 \\
280)\end{array}$ & $\begin{array}{l}141437 \\
(137 \\
606 \text { to } \\
144 \\
793)\end{array}$ & $\begin{array}{l}27 \cdot 25^{*}(22 \cdot 27 \text { to } \\
31 \cdot 31)\end{array}$ & $\begin{array}{l}2001 \cdot 8(1952 \cdot 0 \\
\text { to } 2055 \cdot 9)\end{array}$ & $\begin{array}{l}1885 \cdot 4(1834 \cdot 3 \\
\text { to } 1929 \cdot 7)\end{array}$ & $\begin{array}{l}-5.69 * \\
-2.73)\end{array}$ \\
\hline Chickenpox and herpes zoster & $\begin{array}{l}128020 \\
(126 \\
377 \text { to }\end{array}$ & $\begin{array}{l}139665 \\
(138 \\
706 \text { to }\end{array}$ & $8.84 *(7.73$ to 10.73$)$ & $\begin{array}{l}1992 \cdot 7(1970 \cdot 9 \\
\text { to } 2015 \cdot 0)\end{array}$ & $\begin{array}{l}1935 \cdot 4(1920 \cdot 8 \\
\text { to } 1950 \cdot 4)\end{array}$ & $\begin{array}{l}-2.99 *(-4.00 \text { to } \\
-1.54)\end{array}$ \\
\hline
\end{tabular}




\begin{tabular}{|c|c|c|c|c|c|c|}
\hline & $\begin{array}{l}\text { Cases } \\
\text { in } 1990 \\
(\times \\
1000)\end{array}$ & $\begin{array}{l}\text { Cases } \\
\text { in } 2013 \\
(\times \\
1000)\end{array}$ & Percentage change & $\begin{array}{l}\text { Age- } \\
\text { standardised } \\
\text { rate in } 1990 \\
\text { (per } 100000)\end{array}$ & $\begin{array}{l}\text { Age- } \\
\text { standardised } \\
\text { rate in } 2013 \\
\text { (per } 100000 \text { ) }\end{array}$ & Percentage change \\
\hline & $\begin{array}{l}129 \\
582)\end{array}$ & $\begin{array}{l}140 \\
700)\end{array}$ & & & & \\
\hline Hepatitis B & $\begin{array}{l}137639 \\
(133 \\
533 \text { to } \\
143 \\
049)\end{array}$ & $\begin{array}{l}129191 \\
(124 \\
907 \text { to } \\
132 \\
890)\end{array}$ & $\begin{array}{l}-6 \cdot 22 *(-9.67 \text { to } \\
-2 \cdot 53)\end{array}$ & $\begin{array}{l}2644 \cdot 5(2562 \cdot 1 \\
\text { to } 2753 \cdot 9)\end{array}$ & $\begin{array}{l}1779 \cdot 2(1721 \cdot 7 \\
\text { to } 1830 \cdot 2)\end{array}$ & $\begin{array}{l}-32.74 *(-35.29 \text { to } \\
-29.95)\end{array}$ \\
\hline Gallbladder and biliary diseases & $\begin{array}{l}78635 \\
(77174 \\
\text { to } 80 \\
289)\end{array}$ & $\begin{array}{l}104111 \\
(101 \\
889 \text { to } \\
106 \\
283)\end{array}$ & $\begin{array}{l}32.23 * \\
36.23)\end{array}$ & $\begin{array}{l}2005 \cdot 6(1971 \cdot 1 \\
\text { to } 2049 \cdot 5)\end{array}$ & $\begin{array}{l}1594 \cdot 2(1560 \cdot 6 \\
\text { to } 1627 \cdot 8)\end{array}$ & $\begin{array}{l}-20.56^{*}(-22.64 \text { to } \\
-18.22)\end{array}$ \\
\hline Hepatitis A & $\begin{array}{l}90801 \\
(86969 \\
\text { to } 94 \\
635)\end{array}$ & $\begin{array}{l}101711 \\
(97926 \\
\text { to } 105 \\
499) \\
\end{array}$ & $\begin{array}{l}11 \cdot 87^{*}(11.35 \text { to } \\
12 \cdot 46)\end{array}$ & $\begin{array}{l}1481 \cdot 7(1432 \cdot 5 \\
\text { to } 1529 \cdot 6)\end{array}$ & $\begin{array}{l}1396 \cdot 9(1345 \cdot 4 \\
\text { to } 1448 \cdot 4)\end{array}$ & $\begin{array}{l}-5 \cdot 76^{*}(-6 \cdot 10 \text { to } \\
-5 \cdot 33)\end{array}$ \\
\hline Other unintentional injuries & $\begin{array}{l}70771 \\
(69804 \\
\text { to } 71 \\
730)\end{array}$ & $\begin{array}{l}94747 \\
(93084 \\
\text { to } 96 \\
457)\end{array}$ & $\begin{array}{l}33.88^{*} \text { (31.03 to } \\
36 \cdot 83)\end{array}$ & $\begin{array}{l}1352 \cdot 1(1334 \cdot 1 \\
\text { to } 1370 \cdot 2)\end{array}$ & $\begin{array}{l}1312 \cdot 8(1292 \cdot 3 \\
\text { to } 1334 \cdot 1)\end{array}$ & $\begin{array}{l}-2.90 *(-4.93 \text { to } \\
-0.83)\end{array}$ \\
\hline $\begin{array}{l}\text { Interstitial nephritis and urinary } \\
\text { tract infections }\end{array}$ & $\begin{array}{l}55473 \\
(54702 \\
\text { to } 56 \\
225)\end{array}$ & $\begin{array}{l}92847 \\
(91652 \\
\text { to } 93 \\
940)\end{array}$ & $\begin{array}{l}67 \cdot 10^{*}(64 \cdot 28 \text { to } \\
70 \cdot 38)\end{array}$ & $\begin{array}{l}1156 \cdot 8(1142 \cdot 6 \\
\text { to } 1170 \cdot 9)\end{array}$ & $\begin{array}{l}1344 \cdot 3(1327 \cdot 0 \\
\text { to } 1360 \cdot 3)\end{array}$ & $\begin{array}{l}16 \cdot 14 *(14 \cdot 25 \text { to } \\
18 \cdot 31)\end{array}$ \\
\hline Gastritis and duodenitis & $\begin{array}{l}76611 \\
(75707 \\
\text { to } 77 \\
550)\end{array}$ & $\begin{array}{l}90638 \\
(89750 \\
\text { to } 91 \\
660)\end{array}$ & $\begin{array}{l}18 \cdot 15^{*}(16.30 \text { to } \\
19.94)\end{array}$ & $\begin{array}{l}1809 \cdot 1(1789 \cdot 7 \\
\text { to } 1831 \cdot 4)\end{array}$ & $\begin{array}{l}1393 \cdot 6(1380 \cdot 1 \\
\text { to } 1409 \cdot 5)\end{array}$ & $\begin{array}{l}-23.00^{*}(-24 \cdot 12 \text { to } \\
-21 \cdot 88)\end{array}$ \\
\hline Gonococcal infection & $\begin{array}{l}56316 \\
(53588 \\
\text { to } 59 \\
210)\end{array}$ & $\begin{array}{l}78197 \\
(74585 \\
\text { to } 81 \\
629)\end{array}$ & $\begin{array}{l}39.04 * \\
48.08)\end{array}$ & $\begin{array}{l}977 \cdot 0(931 \cdot 8 \text { to } \\
1024 \cdot 5)\end{array}$ & $\begin{array}{l}1038 \cdot 5(990 \cdot 4 \\
\text { to } 1084 \cdot 2)\end{array}$ & $6.53(-0.93$ to 13.10$)$ \\
\hline Dengue & $\begin{array}{l}8220 \\
(3294 \text { to } \\
17234)\end{array}$ & $\begin{array}{l}58435 \\
(23615 \\
\text { to } 121 \\
951)\end{array}$ & $\begin{array}{l}610 \cdot 87^{*}(606 \cdot 25 \text { to } \\
615 \cdot 50)\end{array}$ & $\begin{array}{l}148 \cdot 3(59 \cdot 4 \text { to } \\
311 \cdot 0)\end{array}$ & $\begin{array}{l}810 \cdot 9(327 \cdot 7 \text { to } \\
1692 \cdot 3)\end{array}$ & $\begin{array}{l}447.31 * \text { (443.59 to } \\
450.91)\end{array}$ \\
\hline Non-venomous animal contact & $\begin{array}{l}65300 \\
(63282 \\
\text { to } 67 \\
643)\end{array}$ & $\begin{array}{l}57822 \\
(55870 \\
\text { to } 59 \\
726)\end{array}$ & $\begin{array}{l}-11.43^{*}(-15.55 \text { to } \\
-7 \cdot 21)\end{array}$ & $\begin{array}{l}1190 \cdot 1(1155 \cdot 7 \\
\text { to } 1228 \cdot 5)\end{array}$ & $\begin{array}{l}808 \cdot 4(782 \cdot 9 \text { to } \\
833 \cdot 1)\end{array}$ & $\begin{array}{l}-32.06^{*}(-34.84 \text { to } \\
-29.13)\end{array}$ \\
\hline Trichomonas infection & $\begin{array}{l}40045 \\
(37105 \\
\text { to } 43 \\
115)\end{array}$ & $\begin{array}{l}57794 \\
(53923 \\
\text { to } 63 \\
336)\end{array}$ & $\begin{array}{l}43.45^{*} \text { (31.00 to } \\
61.93)\end{array}$ & $\begin{array}{l}737 \cdot 0(685 \cdot 1 \text { to } \\
789 \cdot 3)\end{array}$ & $\begin{array}{l}771 \cdot 6(720 \cdot 2 \text { to } \\
845 \cdot 3)\end{array}$ & $4.31(-4.66$ to $17 \cdot 24)$ \\
\hline Motor vehicle road injuries & $\begin{array}{l}40958 \\
(39248 \\
\text { to } 42 \\
998)\end{array}$ & $\begin{array}{l}54201 \\
(51723 \\
\text { to } 57 \\
134)\end{array}$ & $\begin{array}{l}32.35^{*}(28.52 \text { to } \\
37.35)\end{array}$ & $\begin{array}{l}782 \cdot 6(752 \cdot 1 \text { to } \\
821 \cdot 3)\end{array}$ & $\begin{array}{l}763 \cdot 3(729 \cdot 3 \text { to } \\
802 \cdot 9)\end{array}$ & $-2.46(-5.11$ to $0 \cdot 90)$ \\
\hline $\begin{array}{l}\text { Tooth pain due to deciduous } \\
\text { caries }\end{array}$ & $\begin{array}{l}50436 \\
(43544 \\
\text { to } 57 \\
157)\end{array}$ & $\begin{array}{l}53082 \\
(45756 \\
\text { to } 60 \\
269)\end{array}$ & $5.07^{*}(3.77$ to 6.37$)$ & $\begin{array}{l}748 \cdot 9(646 \cdot 6 \text { to } \\
848 \cdot 4)\end{array}$ & $\begin{array}{l}738.4(636 \cdot 5 \text { to } \\
838 \cdot 4)\end{array}$ & $\begin{array}{l}-1.47^{*}(-2.66 \text { to } \\
-0.25)\end{array}$ \\
\hline Peptic ulcer disease & $\begin{array}{l}52264 \\
(51134 \\
\text { to } 53 \\
504)\end{array}$ & $\begin{array}{l}50399 \\
(48835 \\
\text { to } 52 \\
173)\end{array}$ & $-3 \cdot 81(-7 \cdot 37$ to 0.75$)$ & $\begin{array}{l}1457 \cdot 0(1424 \cdot 5 \\
\text { to } 1491 \cdot 1)\end{array}$ & $\begin{array}{l}810 \cdot 7 \text { (786.1 to } \\
838 \cdot 9)\end{array}$ & $\begin{array}{l}-44.43^{*}(-46.54 \text { to } \\
-41.86)\end{array}$ \\
\hline Urolithiasis & $\begin{array}{l}33330 \\
(30306\end{array}$ & $\begin{array}{l}48615 \\
(43576\end{array}$ & $\begin{array}{l}45 \cdot 55^{*} \text { (42.24 to } \\
49 \cdot 34)\end{array}$ & $\begin{array}{l}744 \cdot 9(679 \cdot 4 \text { to } \\
820 \cdot 5)\end{array}$ & $\begin{array}{l}690 \cdot 9(620 \cdot 4 \text { to } \\
767 \cdot 8)\end{array}$ & $\begin{array}{l}-7 \cdot 34^{*}(-9 \cdot 29 \text { to } \\
-5 \cdot 16)\end{array}$ \\
\hline
\end{tabular}




\begin{tabular}{|c|c|c|c|c|c|c|}
\hline & $\begin{array}{l}\text { Cases } \\
\text { in } 1990 \\
(\times \\
1000)\end{array}$ & $\begin{array}{l}\text { Cases } \\
\text { in } 2013 \\
(\times \\
1000)\end{array}$ & Percentage change & $\begin{array}{l}\text { Age- } \\
\text { standardised } \\
\text { rate in } 1990 \\
\text { (per } 100000 \text { ) }\end{array}$ & $\begin{array}{l}\text { Age- } \\
\text { standardised } \\
\text { rate in } 2013 \\
\text { (per } 100000)\end{array}$ & Percentage change \\
\hline & $\begin{array}{l}\text { to } 36 \\
585)\end{array}$ & $\begin{array}{l}\text { to } 54 \\
046)\end{array}$ & & & & \\
\hline Genital herpes & $\begin{array}{l}37033 \\
(35871 \\
\text { to } 38 \\
277)\end{array}$ & $\begin{array}{l}46840 \\
(45386 \\
\text { to } 48 \\
578)\end{array}$ & $\begin{array}{l}26 \cdot 27^{*}(22 \cdot 76 \text { to } \\
30 \cdot 12)\end{array}$ & $\begin{array}{l}633.9(614.0 \text { to } \\
655 \cdot 5)\end{array}$ & $\begin{array}{l}622 \cdot 6(603 \cdot 4 \text { to } \\
645 \cdot 4)\end{array}$ & $-1.85(-4.55$ to 1.08$)$ \\
\hline Cellulitis & $\begin{array}{l}31740 \\
(28667 \\
\text { to } 34 \\
738)\end{array}$ & $\begin{array}{l}37449 \\
(33421 \\
\text { to } 41 \\
180)\end{array}$ & $\begin{array}{l}17 \cdot 85^{*}(13 \cdot 20 \text { to } \\
22 \cdot 04)\end{array}$ & $\begin{array}{l}674 \cdot 3(607 \cdot 1 \text { to } \\
739 \cdot 9)\end{array}$ & $\begin{array}{l}547 \cdot 5(488 \cdot 2 \text { to } \\
604 \cdot 0)\end{array}$ & $\begin{array}{l}-18 \cdot 80^{*} \\
-16 \cdot 80)\end{array}$ \\
\hline Fire, heat, and hot substances & $\begin{array}{l}36843 \\
(35438 \\
\text { to } 38 \\
193)\end{array}$ & $\begin{array}{l}33433 \\
(31570 \\
\text { to } 35 \\
304)\end{array}$ & $\begin{array}{l}-9 \cdot 24^{*}(-14.28 \text { to } \\
-3.71)\end{array}$ & $\begin{array}{l}653.9(631.3 \text { to } \\
677 \cdot 0)\end{array}$ & $\begin{array}{l}450 \cdot 6(426 \cdot 5 \text { to } \\
474 \cdot 6)\end{array}$ & $\begin{array}{l}-31 \cdot 08^{*}(-34 \cdot 68 \text { to } \\
-27 \cdot 22)\end{array}$ \\
\hline Foreign body elsewhere in body & $\begin{array}{l}21835 \\
(21544 \\
\text { to } 22 \\
091)\end{array}$ & $\begin{array}{l}31155 \\
(30749 \\
\text { to } 31 \\
549)\end{array}$ & $\begin{array}{l}42 \cdot 69^{*}(40 \cdot 10 \text { to } \\
45 \cdot 46)\end{array}$ & $\begin{array}{l}455 \cdot 4(450 \cdot 0 \text { to } \\
460 \cdot 8)\end{array}$ & $\begin{array}{l}461 \cdot 0(455 \cdot 3 \text { to } \\
466 \cdot 7)\end{array}$ & $1.23(-0.57$ to 3.05$)$ \\
\hline Hepatitis E & $\begin{array}{l}23967 \\
(22840 \\
\text { to } 24 \\
969)\end{array}$ & $\begin{array}{l}28446 \\
(27083 \\
\text { to } 30 \\
055)\end{array}$ & $\begin{array}{l}18 \cdot 38^{*}(11 \cdot 33 \text { to } \\
26 \cdot 86)\end{array}$ & $\begin{array}{l}433 \cdot 2(414 \cdot 4 \text { to } \\
451 \cdot 1)\end{array}$ & $\begin{array}{l}386 \cdot 8(368 \cdot 4 \text { to } \\
408 \cdot 5)\end{array}$ & $\begin{array}{l}-10 \cdot 88^{*} \text { (-16.18 to } \\
-4 \cdot 62)\end{array}$ \\
\hline $\begin{array}{l}\text { Collective violence and legal } \\
\text { intervention }\end{array}$ & $\begin{array}{l}60427 \\
(40630 \\
\text { to } 98 \\
088)\end{array}$ & $\begin{array}{l}21567 \\
(11959 \\
\text { to } 48 \\
571)\end{array}$ & $\begin{array}{l}-64.08^{*}(-75.95 \text { to } \\
-17.39)\end{array}$ & $\begin{array}{l}1628 \cdot 5(1056 \cdot 4 \\
\text { to } 2651 \cdot 6)\end{array}$ & $\begin{array}{l}390 \cdot 4(229 \cdot 3 \text { to } \\
842 \cdot 7)\end{array}$ & $\begin{array}{l}-75.48 *(-83.87 \text { to } \\
-42.03)\end{array}$ \\
\hline $\begin{array}{l}\text { Adverse effects of medical } \\
\text { treatment }\end{array}$ & $\begin{array}{l}13489 \\
(13334 \\
\text { to } 13 \\
646)\end{array}$ & $\begin{array}{l}19946 \\
(19714 \\
\text { to } 20 \\
176)\end{array}$ & $\begin{array}{l}47 \cdot 88^{*}(45 \cdot 68 \text { to } \\
50 \cdot 11)\end{array}$ & $\begin{array}{l}273 \cdot 3(270 \cdot 2 \text { to } \\
276 \cdot 4)\end{array}$ & $\begin{array}{l}269 \cdot 1(266 \cdot 3 \text { to } \\
271 \cdot 9)\end{array}$ & $\begin{array}{l}-1.52^{*}(-2.93 \text { to } \\
-0.16)\end{array}$ \\
\hline Assault by other means & $\begin{array}{l}16174 \\
(15804 \\
\text { to } 16 \\
557)\end{array}$ & $\begin{array}{l}18133 \\
(17708 \\
\text { to } 18 \\
553)\end{array}$ & $\begin{array}{l}12 \cdot 12^{*}(9 \cdot 14 \text { to } \\
15 \cdot 10)\end{array}$ & $\begin{array}{l}299 \cdot 4(292 \cdot 7 \text { to } \\
306 \cdot 3)\end{array}$ & $\begin{array}{l}249 \cdot 2(243 \cdot 6 \text { to } \\
254 \cdot 9)\end{array}$ & $\begin{array}{l}-16 \cdot 76^{*} \\
-14.50)\end{array}$ \\
\hline Other transport injuries & $\begin{array}{l}16956 \\
(16240 \\
\text { to } 18 \\
001)\end{array}$ & $\begin{array}{l}18083 \\
(17246 \\
\text { to } 19 \\
205)\end{array}$ & $6.70^{*}(0.04$ to 13.62$)$ & $\begin{array}{l}315 \cdot 7(302 \cdot 8 \text { to } \\
332 \cdot 9)\end{array}$ & $\begin{array}{l}244 \cdot 5(233 \cdot 3 \text { to } \\
259 \cdot 8)\end{array}$ & $\begin{array}{l}-22 \cdot 50 *(-27 \cdot 11 \text { to } \\
-17 \cdot 66)\end{array}$ \\
\hline Pancreatitis & $\begin{array}{l}10057 \\
(9954 \text { to } \\
10157)\end{array}$ & $\begin{array}{l}17163 \\
(16976 \\
\text { to } 17 \\
376)\end{array}$ & $\begin{array}{l}70 \cdot 45^{*}(68 \cdot 14 \text { to } \\
72 \cdot 80)\end{array}$ & $\begin{array}{l}234 \cdot 8(232 \cdot 6 \text { to } \\
237 \cdot 1)\end{array}$ & $\begin{array}{l}251 \cdot 0(248.3 \text { to } \\
253 \cdot 9)\end{array}$ & $6.84 *(5.45$ to 8.28$)$ \\
\hline Motorcyclist road injuries & $\begin{array}{l}14619 \\
(12221 \\
\text { to } 16 \\
302)\end{array}$ & $\begin{array}{l}16692 \\
(13485 \\
\text { to } 18 \\
844)\end{array}$ & $\begin{array}{l}14 \cdot 15^{*} \text { (7.74 to } \\
21.46)\end{array}$ & $\begin{array}{l}273.5(227.9 \text { to } \\
305 \cdot 0)\end{array}$ & $\begin{array}{l}226 \cdot 9(184 \cdot 0 \text { to } \\
256 \cdot 2)\end{array}$ & $\begin{array}{l}-17 \cdot 08^{*} \text { ( }-21.40 \text { to } \\
-11.90)\end{array}$ \\
\hline Appendicitis & $\begin{array}{l}14105 \\
(12914 \\
\text { to } 15 \\
603)\end{array}$ & $\begin{array}{l}16423 \\
(14441 \\
\text { to } 18 \\
501)\end{array}$ & $\begin{array}{l}16 \cdot 60 *(0 \cdot 89 \text { to } \\
36 \cdot 82)\end{array}$ & $\begin{array}{l}263.6(241.9 \text { to } \\
290.9)\end{array}$ & $\begin{array}{l}225 \cdot 2(198 \cdot 5 \text { to } \\
253 \cdot 1)\end{array}$ & $\begin{array}{l}-14.58(-25.48 \text { to } \\
0.15)\end{array}$ \\
\hline $\begin{array}{l}\text { Pedestrian road injuries by road } \\
\text { vehicle }\end{array}$ & $\begin{array}{l}10450 \\
(9702 \text { to } \\
11340)\end{array}$ & $\begin{array}{l}14353 \\
(13302 \\
\text { to } 15 \\
545)\end{array}$ & $\begin{array}{l}37.38 * \text { (32.69 to } \\
43.65)\end{array}$ & $\begin{array}{l}209 \cdot 8(194 \cdot 3 \text { to } \\
228 \cdot 1)\end{array}$ & $\begin{array}{l}210 \cdot 5(194 \cdot 8 \text { to } \\
227 \cdot 5)\end{array}$ & $0.35(-3.12$ to 4.53$)$ \\
\hline $\begin{array}{l}\text { Meningitis cases due to other } \\
\text { causes }\end{array}$ & $\begin{array}{l}14177 \\
(13756 \\
\text { to } 14 \\
595)\end{array}$ & $\begin{array}{l}12819 \\
(12410 \\
\text { to } 13 \\
260)\end{array}$ & $\begin{array}{l}-9.58 *(-13.65 \text { to } \\
-5.73)\end{array}$ & $\begin{array}{l}224.6(217.9 \text { to } \\
231.5)\end{array}$ & $\begin{array}{l}175 \cdot 3(169 \cdot 7 \text { to } \\
181 \cdot 2)\end{array}$ & $\begin{array}{l}-21.89 * \\
-18 \cdot 63)\end{array}$ \\
\hline
\end{tabular}




\begin{tabular}{|c|c|c|c|c|c|c|}
\hline & $\begin{array}{l}\text { Cases } \\
\text { in } 1990 \\
(x \\
1000)\end{array}$ & $\begin{array}{l}\text { Cases } \\
\text { in } 2013 \\
(x \\
1000)\end{array}$ & Percentage change & $\begin{array}{l}\text { Age- } \\
\text { standardised } \\
\text { rate in } 1990 \\
(\text { per } 100000)\end{array}$ & $\begin{array}{l}\text { Age- } \\
\text { standardised } \\
\text { rate in } 2013 \\
(\text { per } 100000)\end{array}$ & Percentage change \\
\hline Typhoid fever & $\begin{array}{l}13685 \\
(11708 \\
\text { to } 17 \\
982)\end{array}$ & $\begin{array}{l}10955 \\
(9641 \text { to } \\
14354)\end{array}$ & $\begin{array}{l}-19.93^{*} \text { (-30.68 to } \\
-6.53)\end{array}$ & $\begin{array}{l}227 \cdot 3(195 \cdot 5 \text { to } \\
297 \cdot 7)\end{array}$ & $\begin{array}{l}151.5(133.6 \text { to } \\
198.5)\end{array}$ & $\begin{array}{l}-33.30 * \text { ( }-41.90 \text { to } \\
-22 \cdot 36)\end{array}$ \\
\hline Hepatitis C & $\begin{array}{l}9367 \\
(9188 \text { to } \\
9539)\end{array}$ & $\begin{array}{l}10840 \\
(10650 \\
\text { to } 11 \\
014)\end{array}$ & $\begin{array}{l}15 \cdot 54^{*} \\
19 \cdot 02)\end{array}$ & $\begin{array}{l}201 \cdot 0(196 \cdot 3 \text { to } \\
205 \cdot 6)\end{array}$ & $\begin{array}{l}157 \cdot 7(154.7 \text { to } \\
160 \cdot 5)\end{array}$ & $\begin{array}{l}-21.59^{*}(-23.94 \text { to } \\
-18.90)\end{array}$ \\
\hline Cyclist road injuries & $\begin{array}{l}9713 \\
(8578 \text { to } \\
10973)\end{array}$ & $\begin{array}{l}10711 \\
(9478 \text { to } \\
12157)\end{array}$ & $\begin{array}{l}10 \cdot 30 *(5 \cdot 21 \text { to } \\
15 \cdot 85)\end{array}$ & $\begin{array}{l}183 \cdot 8(164 \cdot 6 \text { to } \\
205 \cdot 9)\end{array}$ & $\begin{array}{l}152.9(135.9 \text { to } \\
173.6)\end{array}$ & $\begin{array}{l}-16 \cdot 84^{*} \\
-13 \cdot 23)\end{array}$ \\
\hline Acute myocardial infarction & $\begin{array}{l}4862 \\
(4640 \text { to } \\
5074)\end{array}$ & $\begin{array}{l}8557 \\
(8199 \text { to } \\
8919)\end{array}$ & $\begin{array}{l}75 \cdot 77^{*}(74 \cdot 24 \text { to } \\
77 \cdot 72)\end{array}$ & $\begin{array}{l}141 \cdot 1(134 \cdot 6 \text { to } \\
147 \cdot 6)\end{array}$ & $\begin{array}{l}139 \cdot 3(133 \cdot 2 \text { to } \\
145 \cdot 4)\end{array}$ & $\begin{array}{l}-1 \cdot 29^{*}(-2 \cdot 09 \text { to } \\
-0.34)\end{array}$ \\
\hline Assault by sharp object & $\begin{array}{l}6021 \\
(5779 \text { to } \\
6234)\end{array}$ & $\begin{array}{l}7983 \\
\text { (7641 to } \\
8284)\end{array}$ & $\begin{array}{l}32 \cdot 62 *(26.92 \text { to } \\
38 \cdot 28)\end{array}$ & $\begin{array}{l}111 \cdot 8(107 \cdot 2 \text { to } \\
115 \cdot 6)\end{array}$ & $\begin{array}{l}107 \cdot 6(103 \cdot 1 \text { to } \\
111.5)\end{array}$ & $-3.75(-7.87$ to 0.21$)$ \\
\hline Acute ischaemic stroke & $\begin{array}{l}4309 \\
(4118 \text { to } \\
4532)\end{array}$ & $\begin{array}{l}6893 \\
(6550 \text { to } \\
7352)\end{array}$ & $\begin{array}{l}59 \cdot 61 *(48 \cdot 61 \text { to } \\
72 \cdot 86)\end{array}$ & $\begin{array}{l}128 \cdot 4(122 \cdot 7 \text { to } \\
135 \cdot 0)\end{array}$ & $\begin{array}{l}114 \cdot 3(108 \cdot 5 \text { to } \\
122 \cdot 3)\end{array}$ & $\begin{array}{l}-11 \cdot 13^{*}(-17 \cdot 29 \text { to } \\
-3 \cdot 14)\end{array}$ \\
\hline Paratyphoid fever & $\begin{array}{l}8846 \\
\text { (7761 to } \\
10194)\end{array}$ & $\begin{array}{l}6378 \\
(5550 \text { to } \\
7253)\end{array}$ & $\begin{array}{l}-27.88^{*}(-41.81 \text { to } \\
-12.83)\end{array}$ & $\begin{array}{l}144 \cdot 5(127 \cdot 5 \text { to } \\
166 \cdot 1)\end{array}$ & $\begin{array}{l}88 \cdot 0(76 \cdot 5 \text { to } \\
100 \cdot 0)\end{array}$ & $\begin{array}{l}-39.09^{*}(-50 \cdot 86 \text { to } \\
-26.48)\end{array}$ \\
\hline Maternal hypertensive disorders & $\begin{array}{l}5582 \\
(3681 \text { to } \\
7427)\end{array}$ & $\begin{array}{l}5707 \\
(3792 \text { to } \\
7519)\end{array}$ & $2.08(-1.25$ to $7 \cdot 48)$ & $\begin{array}{l}96.7(63.9 \text { to } \\
127.8)\end{array}$ & $\begin{array}{l}75 \cdot 2(50 \cdot 1 \text { to } \\
98 \cdot 9)\end{array}$ & $\begin{array}{l}-22 \cdot 26^{*}(-24 \cdot 37 \text { to } \\
-18 \cdot 54)\end{array}$ \\
\hline Exposure to forces of nature & $\begin{array}{l}7326 \\
(4728 \text { to } \\
13492)\end{array}$ & $\begin{array}{l}5658 \\
(3694 \text { to } \\
11899)\end{array}$ & $\begin{array}{l}-21.55(-45 \cdot 57 \text { to } \\
31 \cdot 37)\end{array}$ & $\begin{array}{l}195 \cdot 7(114.9 \text { to } \\
333 \cdot 1)\end{array}$ & $\begin{array}{l}97 \cdot 3(55 \cdot 2 \text { to } \\
187 \cdot 5)\end{array}$ & $\begin{array}{l}-50.75^{*} \text { (-71.91 to } \\
-9.97)\end{array}$ \\
\hline Venomous animal contact & $\begin{array}{l}5702 \\
(5390 \text { to } \\
6040)\end{array}$ & $\begin{array}{l}5548 \\
(5278 \text { to } \\
5843)\end{array}$ & $-2.68(-6.78$ to 1.46$)$ & $\begin{array}{l}106 \cdot 0(101 \cdot 0 \text { to } \\
111 \cdot 4)\end{array}$ & $\begin{array}{l}77 \cdot 1(73 \cdot 7 \text { to } \\
80 \cdot 8)\end{array}$ & $\begin{array}{l}-27.28^{*} \text { (-29.90 to } \\
-24.43)\end{array}$ \\
\hline Foreign body in eyes & $\begin{array}{l}3959 \\
(3692 \text { to } \\
4201)\end{array}$ & $\begin{array}{l}5298 \\
(4955 \text { to } \\
5607)\end{array}$ & $\begin{array}{l}33 \cdot 81 *(30 \cdot 26 \text { to } \\
37 \cdot 76)\end{array}$ & $\begin{array}{l}74 \cdot 6(69 \cdot 6 \text { to } \\
79 \cdot 1)\end{array}$ & $\begin{array}{l}73.5(68.6 \text { to } \\
77.7)\end{array}$ & $-1.39(-3.97$ to 1.45$)$ \\
\hline Obstructed labour & $\begin{array}{l}5362 \\
(4833 \text { to } \\
5947)\end{array}$ & $\begin{array}{l}5122 \\
(4491 \text { to } \\
5610)\end{array}$ & $\begin{array}{l}-4 \cdot 23(-19 \cdot 90 \text { to } \\
10 \cdot 21)\end{array}$ & $\begin{array}{l}93 \cdot 7(84 \cdot 8 \text { to } \\
103 \cdot 6)\end{array}$ & $\begin{array}{l}67 \cdot 2(59 \cdot 0 \text { to } \\
73 \cdot 6)\end{array}$ & $\begin{array}{l}-27.99^{*}(-39.42 \text { to } \\
-17.41)\end{array}$ \\
\hline Maternal haemorrhage & $\begin{array}{l}4429 \\
(3994 \text { to } \\
5014)\end{array}$ & $\begin{array}{l}4649 \\
(4331 \text { to } \\
5015)\end{array}$ & $4.72(-7.62$ to 17.74$)$ & $\begin{array}{l}77 \cdot 6(70 \cdot 3 \text { to } \\
87 \cdot 3)\end{array}$ & $\begin{array}{l}61 \cdot 1(57 \cdot 0 \text { to } \\
65.9)\end{array}$ & $\begin{array}{l}-21 \cdot 40^{*}(-30 \cdot 27 \text { to } \\
-11 \cdot 77)\end{array}$ \\
\hline Assault by firearm & $\begin{array}{l}2487 \\
(2266 \text { to } \\
2671)\end{array}$ & $\begin{array}{l}3609 \\
(3313 \text { to } \\
3881)\end{array}$ & $\begin{array}{l}45 \cdot 16^{*}(39.56 \text { to } \\
51 \cdot 09)\end{array}$ & $\begin{array}{l}48 \cdot 2(44 \cdot 0 \text { to } \\
51 \cdot 7)\end{array}$ & $\begin{array}{l}50 \cdot 0(46 \cdot 0 \text { to } \\
53 \cdot 8)\end{array}$ & $3.77(-0.17$ to 7.93$)$ \\
\hline $\begin{array}{l}\text { Pulmonary aspiration and } \\
\text { foreign body in airway }\end{array}$ & $\begin{array}{l}2777 \\
(2639 \text { to } \\
2986)\end{array}$ & $\begin{array}{l}3568 \\
(3396 \text { to } \\
3832)\end{array}$ & $\begin{array}{l}28 \cdot 50 * \\
31 \cdot 45)\end{array}$ (25.60 to & $\begin{array}{l}52.5(49 \cdot 9 \text { to } \\
56 \cdot 3)\end{array}$ & $\begin{array}{l}49 \cdot 7(47 \cdot 4 \text { to } \\
53 \cdot 4)\end{array}$ & $\begin{array}{l}-5 \cdot 28^{*}(-7 \cdot 23 \text { to } \\
-3 \cdot 18)\end{array}$ \\
\hline Acute haemorrhagic stroke & $\begin{array}{l}1886 \\
(1817 \text { to } \\
1977)\end{array}$ & $\begin{array}{l}3366 \\
(3200 \text { to } \\
3543)\end{array}$ & $\begin{array}{l}78.50 *(65.50 \text { to } \\
89.81)\end{array}$ & $\begin{array}{l}53.4(51.5 \text { to } \\
55.9)\end{array}$ & $\begin{array}{l}54 \cdot 3(51 \cdot 4 \text { to } \\
57 \cdot 3)\end{array}$ & $1.61(-5.68$ to 8.55$)$ \\
\hline Poisonings & $\begin{array}{l}3409 \\
\text { (3347 to } \\
3477)\end{array}$ & $\begin{array}{l}3282 \\
\text { (3208 to } \\
3352)\end{array}$ & $\begin{array}{l}-3 \cdot 70^{*}(-6 \cdot 17 \text { to } \\
-1 \cdot 39)\end{array}$ & $\begin{array}{l}61 \cdot 4(60 \cdot 3 \text { to } \\
62 \cdot 5)\end{array}$ & $\begin{array}{l}44.6(43.6 \text { to } \\
45.5)\end{array}$ & $\begin{array}{l}-27.43 *(-29.23 \text { to } \\
-25.83)\end{array}$ \\
\hline
\end{tabular}




\begin{tabular}{|c|c|c|c|c|c|c|}
\hline & $\begin{array}{l}\text { Cases } \\
\text { in } 1990 \\
(\times \\
1000)\end{array}$ & $\begin{array}{l}\text { Cases } \\
\text { in } 2013 \\
(\times \\
1000)\end{array}$ & Percentage change & $\begin{array}{l}\text { Age- } \\
\text { standardised } \\
\text { rate in } 1990 \\
\text { (per } 100000 \text { ) }\end{array}$ & $\begin{array}{l}\text { Age- } \\
\text { standardised } \\
\text { rate in } 2013 \\
\text { (per } 100000 \text { ) }\end{array}$ & Percentage change \\
\hline Self-harm & $\begin{array}{l}3222 \\
(3187 \text { to } \\
3257)\end{array}$ & $\begin{array}{l}3270 \\
(3240 \text { to } \\
3302)\end{array}$ & $1.51^{*}(0.20$ to $2 \cdot 79)$ & $\begin{array}{l}61 \cdot 2(60 \cdot 6 \text { to } \\
61 \cdot 8)\end{array}$ & $\begin{array}{l}43 \cdot 8(43 \cdot 3 \text { to } \\
44 \cdot 2)\end{array}$ & $\begin{array}{l}-28.48^{*}(-29.34 \text { to } \\
-27.61)\end{array}$ \\
\hline Unintentional firearm injuries & $\begin{array}{l}2749 \\
(2556 \text { to } \\
2925)\end{array}$ & $\begin{array}{l}3126 \\
(2902 \text { to } \\
3344)\end{array}$ & $\begin{array}{l}13.71 *(10 \cdot 07 \text { to } \\
17 \cdot 66)\end{array}$ & $\begin{array}{l}49 \cdot 4(46 \cdot 0 \text { to } \\
52 \cdot 5)\end{array}$ & $\begin{array}{l}42.7(39 \cdot 7 \text { to } \\
45 \cdot 6)\end{array}$ & $\begin{array}{l}-13 \cdot 66^{*}(-16 \cdot 30 \text { to } \\
-10 \cdot 85)\end{array}$ \\
\hline Complications of abortion & $\begin{array}{l}2366 \\
(2235 \text { to } \\
2525)\end{array}$ & $\begin{array}{l}2642 \\
(2510 \text { to } \\
2784)\end{array}$ & $\begin{array}{l}11.54^{*}(1.79 \text { to } \\
20.91)\end{array}$ & $\begin{array}{l}41 \cdot 9(39 \cdot 6 \text { to } \\
44 \cdot 6)\end{array}$ & $\begin{array}{l}34 \cdot 9(33 \cdot 1 \text { to } \\
36 \cdot 7)\end{array}$ & $\begin{array}{l}-16 \cdot 87^{*}(-24 \cdot 01 \text { to } \\
-10 \cdot 06)\end{array}$ \\
\hline $\begin{array}{l}\text { Paralytic ileus and intestinal } \\
\text { obstruction }\end{array}$ & $\begin{array}{l}1501 \\
(1481 \text { to } \\
1517)\end{array}$ & $\begin{array}{l}2530 \\
(2487 \text { to } \\
2572)\end{array}$ & $\begin{array}{l}68 \cdot 38^{*}(64.90 \text { to } \\
71 \cdot 76)\end{array}$ & $\begin{array}{l}38.1(37.5 \text { to } \\
38 \cdot 5)\end{array}$ & $\begin{array}{l}39 \cdot 6(38 \cdot 9 \text { to } \\
40 \cdot 3)\end{array}$ & $4.04^{*}(1.81$ to 6.20$)$ \\
\hline $\begin{array}{l}\text { Other sexually transmitted } \\
\text { diseases }\end{array}$ & $\begin{array}{l}1884 \\
(1771 \text { to } \\
2008)\end{array}$ & $\begin{array}{l}2393 \\
(2259 \text { to } \\
2540)\end{array}$ & $\begin{array}{l}27 \cdot 09^{*}(17 \cdot 35 \text { to } \\
36 \cdot 22)\end{array}$ & $\begin{array}{l}36 \cdot 1(34 \cdot 0 \text { to } \\
38 \cdot 3)\end{array}$ & $\begin{array}{l}32 \cdot 3(30 \cdot 6 \text { to } \\
34 \cdot 3)\end{array}$ & $\begin{array}{l}-10 \cdot 14^{*} \\
-3 \cdot 89)\end{array}$ \\
\hline Drowning & $\begin{array}{l}1803 \\
(1708 \text { to } \\
1921)\end{array}$ & $\begin{array}{l}1664 \\
(1571 \text { to } \\
1782)\end{array}$ & $\begin{array}{l}-7.70 *(-11.01 \text { to } \\
-4 \cdot 22)\end{array}$ & $\begin{array}{l}32 \cdot 5(30 \cdot 8 \text { to } \\
34 \cdot 7)\end{array}$ & $\begin{array}{l}23 \cdot 6(22 \cdot 3 \text { to } \\
25 \cdot 3)\end{array}$ & $\begin{array}{l}-27.33^{*}(-30.05 \text { to } \\
-24 \cdot 60)\end{array}$ \\
\hline Unintentional suffocation & $\begin{array}{l}1102 \\
(1003 \text { to } \\
1205)\end{array}$ & $\begin{array}{l}1593 \\
(1443 \text { to } \\
1741)\end{array}$ & $\begin{array}{l}44 \cdot 53^{*} \text { (40.31 to } \\
48 \cdot 18)\end{array}$ & $\begin{array}{l}19 \cdot 4(17 \cdot 7 \text { to } \\
21 \cdot 2)\end{array}$ & $\begin{array}{l}21 \cdot 8(19 \cdot 7 \text { to } \\
23 \cdot 8)\end{array}$ & $\begin{array}{l}12 \cdot 17^{*}(9 \cdot 16 \text { to } \\
14.93)\end{array}$ \\
\hline Acute myocarditis & $\begin{array}{l}961 \\
(909 \text { to } \\
1031)\end{array}$ & $\begin{array}{l}1481 \\
(1377 \text { to } \\
1592)\end{array}$ & $\begin{array}{l}53 \cdot 54^{*}(41.58 \text { to } \\
69 \cdot 41)\end{array}$ & $\begin{array}{l}22 \cdot 8(21 \cdot 5 \text { to } \\
24 \cdot 5)\end{array}$ & $\begin{array}{l}22 \cdot 0(20 \cdot 5 \text { to } \\
23 \cdot 6)\end{array}$ & $\begin{array}{l}-3 \cdot 56(-11 \cdot 38 \text { to } \\
5 \cdot 97)\end{array}$ \\
\hline $\begin{array}{l}\text { Maternal sepsis and other } \\
\text { maternal infections }\end{array}$ & $\begin{array}{l}1881 \\
(1684 \text { to } \\
2088)\end{array}$ & $\begin{array}{l}1389 \\
(1227 \text { to } \\
1522)\end{array}$ & $\begin{array}{l}-26 \cdot 48 *(-36 \cdot 40 \text { to } \\
-15 \cdot 31)\end{array}$ & $\begin{array}{l}32 \cdot 2(28 \cdot 9 \text { to } \\
35 \cdot 6)\end{array}$ & $\begin{array}{l}18 \cdot 3(16 \cdot 2 \text { to } \\
20 \cdot 0)\end{array}$ & $\begin{array}{l}-43 \cdot 42 *(-50 \cdot 97 \text { to } \\
-34.94)\end{array}$ \\
\hline
\end{tabular}

Causes are ordered by overall incidence. All data are shown with $95 \%$ uncertainty intervals.

Significant percentage change. 


\begin{tabular}{|c|c|c|c|c|c|c|c|c|c|c|c|c|c|c|c|}
\hline 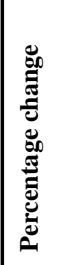 & 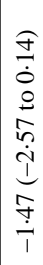 & 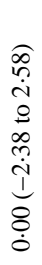 & 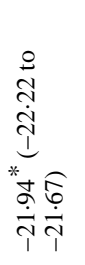 & 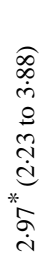 & 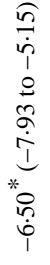 & 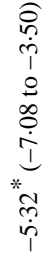 & 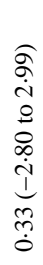 & 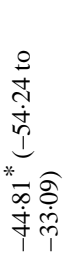 & 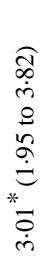 & 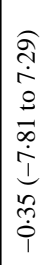 & 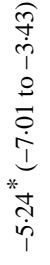 & 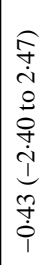 & 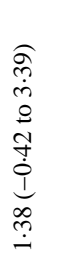 & 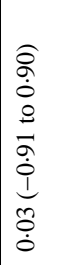 & 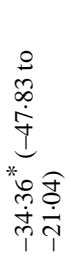 \\
\hline
\end{tabular}

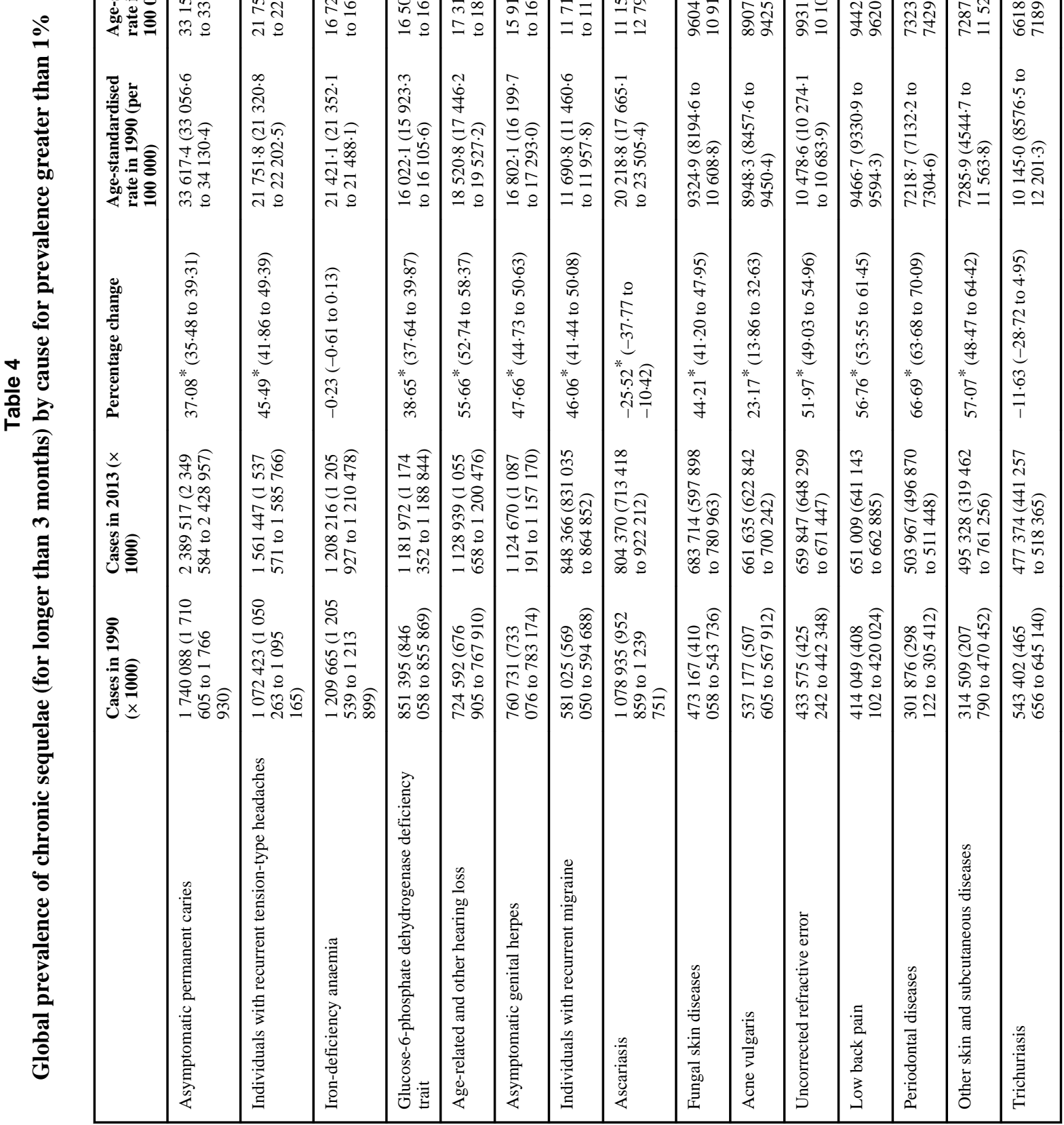




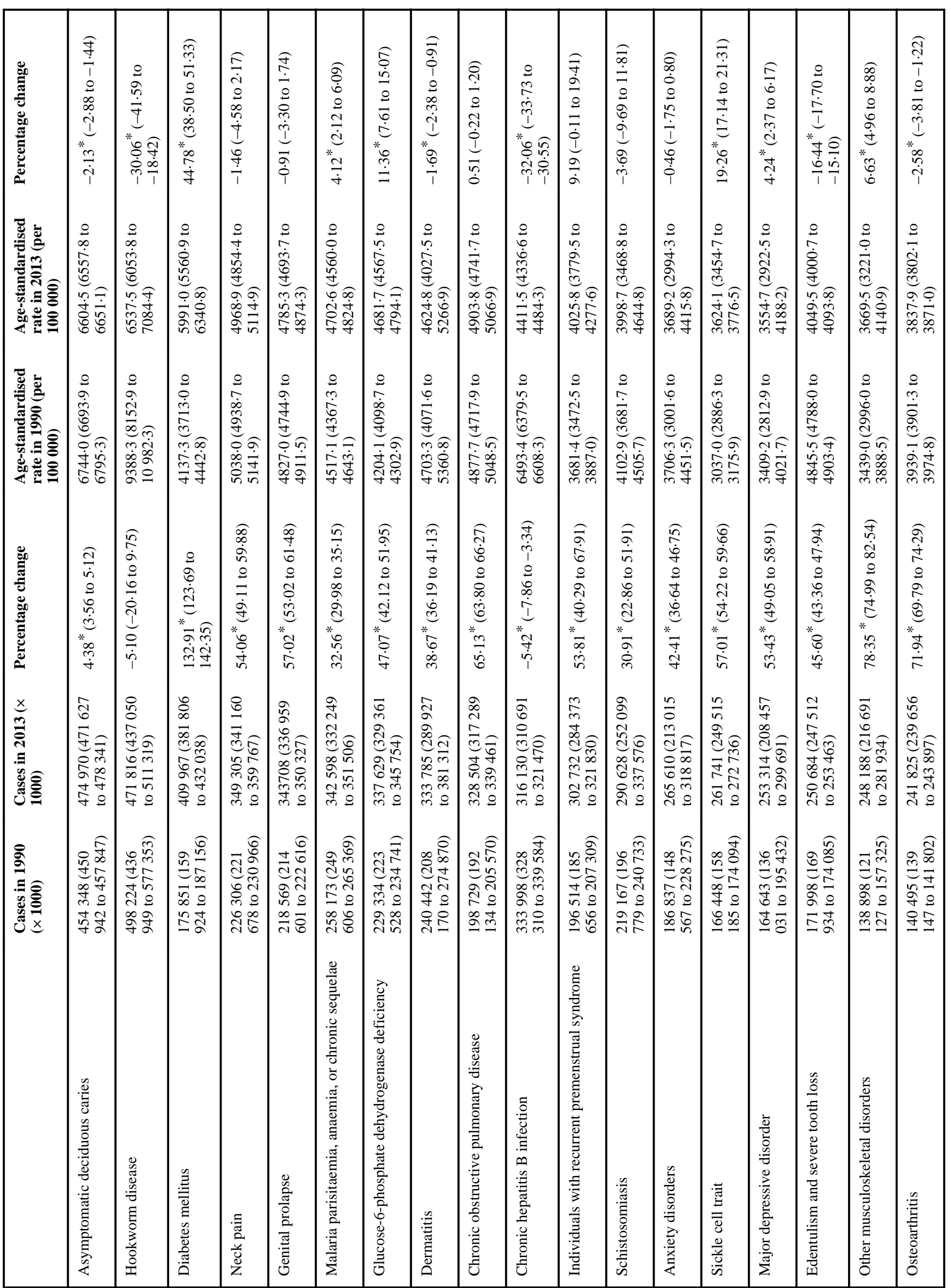




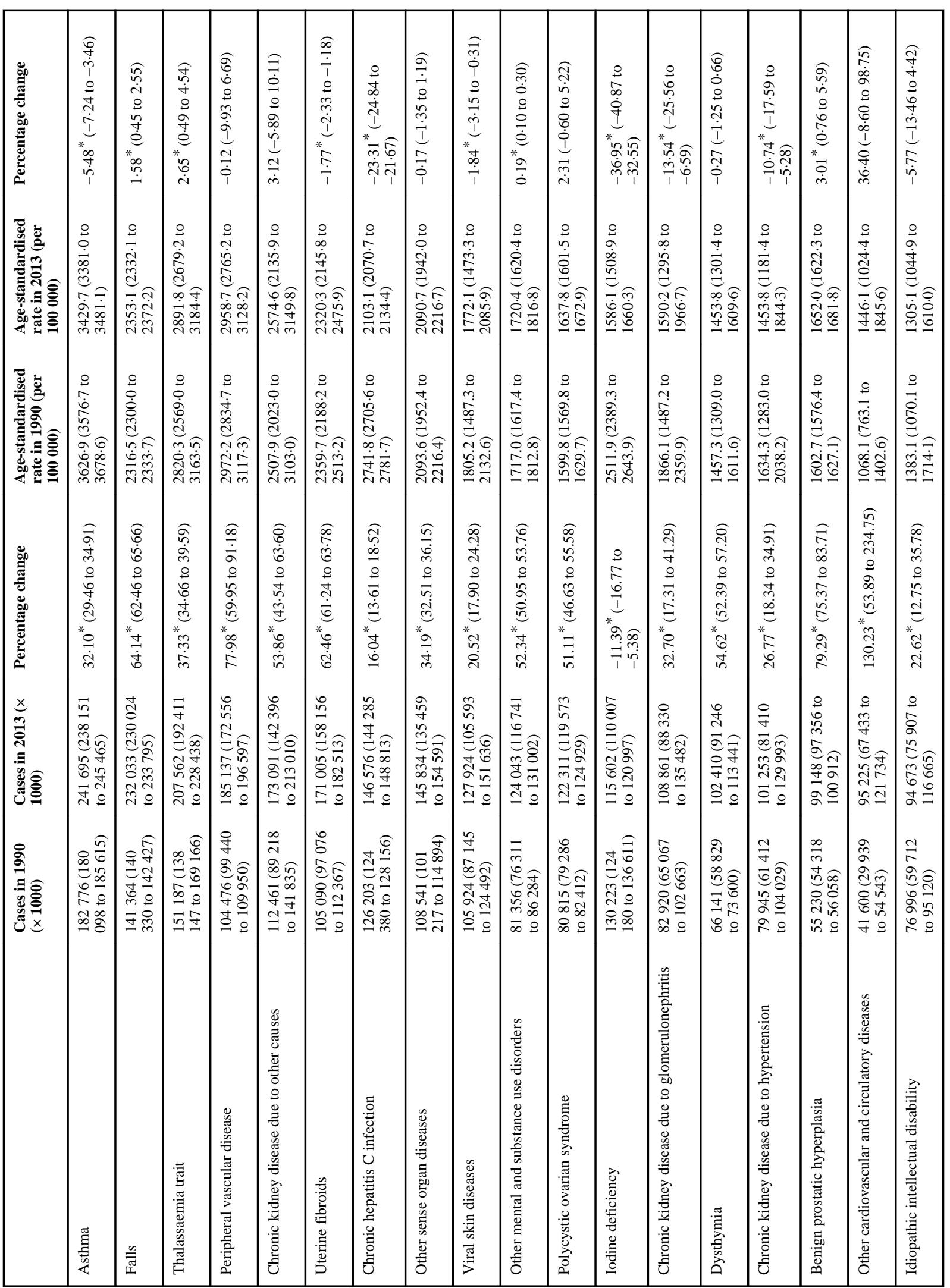




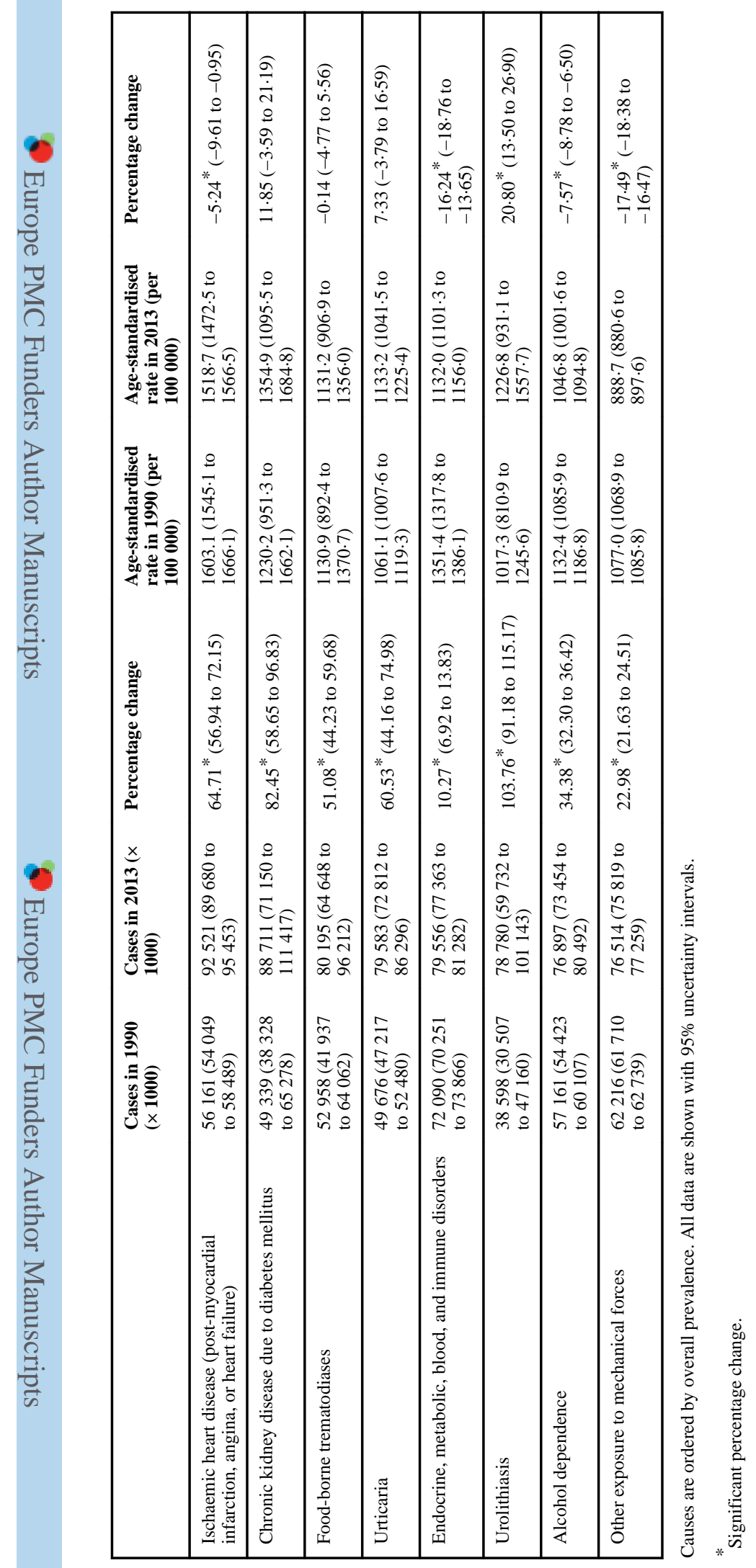


Table 5

Prevalence and YLDs, with percentage of total, for anaemia by cause in 1990 and 2013, and prevalence by severity in 2013

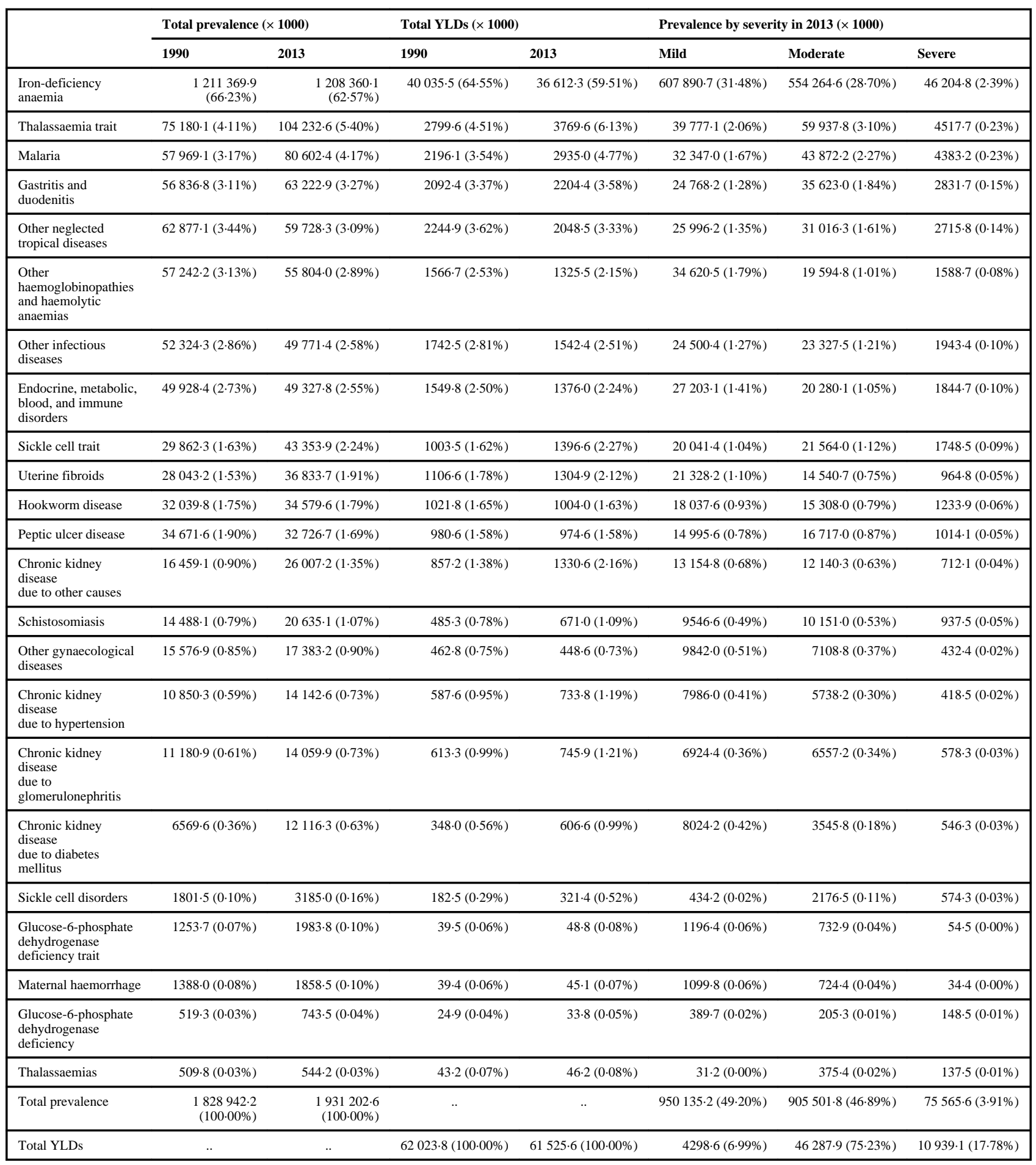

Data are number $(\%)$. YLDs=years lived with disability. 


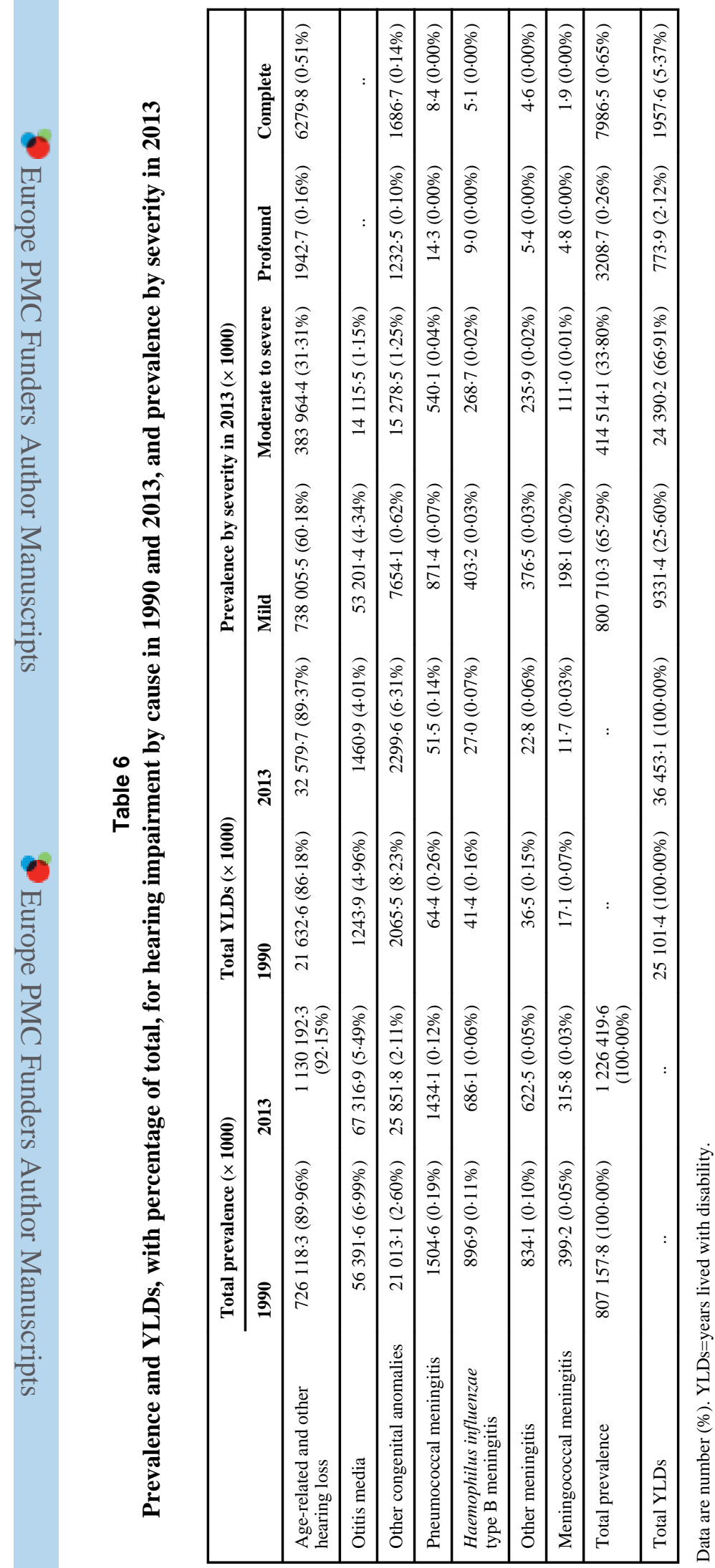

Lancet. Author manuscript; available in PMC 2015 September 07. 
Table 7

Prevalence and YLDs, with percentage of total, for heart failure by cause in 1990 and 2013, and prevalence by severity in 2013

\begin{tabular}{|c|c|c|c|c|c|c|c|}
\hline & \multicolumn{2}{|c|}{ Total prevalence $(\times 1000)$} & \multicolumn{2}{|c|}{ Total YLDs $(\times 1000)$} & \multicolumn{3}{|c|}{ Prevalence by severity in $2013(\times 1000)$} \\
\hline & 1990 & 2013 & 1990 & 2013 & Mild & Moderate & Severe \\
\hline Ischaemic heart disease & $10298.9(32.79 \%)$ & $20372.6(33.04 \%)$ & $1114 \cdot 3(25 \cdot 51 \%)$ & $2216 \cdot 1(25 \cdot 89 \%)$ & $5055 \cdot 3(8 \cdot 20 \%)$ & $4131 \cdot 3(6 \cdot 70 \%)$ & $11186 \cdot 1(18 \cdot 14 \%)$ \\
\hline $\begin{array}{l}\text { Hypertensive heart } \\
\text { disease }\end{array}$ & $5128 \cdot 4(16 \cdot 33 \%)$ & $10906 \cdot 9(17.69 \%)$ & $559 \cdot 1(12 \cdot 80 \%)$ & $1193.9(13.95 \%)$ & $2707 \cdot 3(4 \cdot 39 \%)$ & $2215 \cdot 8(3.59 \%)$ & $5983.8(9 \cdot 70 \%)$ \\
\hline $\begin{array}{l}\text { Other cardiovascular } \\
\text { and circulatory } \\
\text { diseases }\end{array}$ & $4117 \cdot 6(13 \cdot 11 \%)$ & $9542 \cdot 1(15 \cdot 48 \%)$ & $448 \cdot 9(10 \cdot 28 \%)$ & $1042 \cdot 6(12 \cdot 18 \%)$ & $2356 \cdot 1(3 \cdot 82 \%)$ & $1945 \cdot 8(3 \cdot 16 \%)$ & $5240 \cdot 3(8 \cdot 50 \%)$ \\
\hline $\begin{array}{l}\text { Cardiomyopathy and } \\
\text { myocarditis }\end{array}$ & $4077 \cdot 6(12.98 \%)$ & $7629.9(12.37 \%)$ & $451.9(10 \cdot 35 \%)$ & $847.9(9.91 \%)$ & $1880 \cdot 1(3.05 \%)$ & $1553.8(2.52 \%)$ & $4196 \cdot 1(6 \cdot 81 \%)$ \\
\hline $\begin{array}{l}\text { Chronic obstructive } \\
\text { pulmonary disease }\end{array}$ & $3036 \cdot 5(9 \cdot 67 \%)$ & $5846 \cdot 4(9 \cdot 48 \%)$ & $1222.0(27.98 \%)$ & $2366 \cdot 4(27.65 \%)$ & $1438.2(2 \cdot 33 \%)$ & $1201.5(1.95 \%)$ & $3206 \cdot 8(5 \cdot 20 \%)$ \\
\hline $\begin{array}{l}\text { Rheumatic heart } \\
\text { disease }\end{array}$ & $2837.8(9 \cdot 04 \%)$ & $4274.0(6.93 \%)$ & $316 \cdot 2(7 \cdot 24 \%)$ & $473 \cdot 2(5 \cdot 53 \%)$ & $1055 \cdot 5(1.71 \%)$ & $873 \cdot 1(1 \cdot 42 \%)$ & $2345 \cdot 5(3 \cdot 80 \%)$ \\
\hline $\begin{array}{l}\text { Congenital heart } \\
\text { anomalies }\end{array}$ & $495.9(1.58 \%)$ & $621.7(1.01 \%)$ & $60 \cdot 0(1 \cdot 37 \%)$ & $74 \cdot 8(0 \cdot 87 \%)$ & $152 \cdot 2(0 \cdot 25 \%)$ & $126 \cdot 8(0 \cdot 21 \%)$ & $342 \cdot 7(0 \cdot 56 \%)$ \\
\hline $\begin{array}{l}\text { Iron-deficiency } \\
\text { anaemia }\end{array}$ & $373 \cdot 2(1 \cdot 19 \%)$ & $446 \cdot 6(0 \cdot 72 \%)$ & $43 \cdot 1(0 \cdot 99 \%)$ & $51 \cdot 2(0 \cdot 60 \%)$ & $110 \cdot 1(0 \cdot 18 \%)$ & $90 \cdot 8(0 \cdot 15 \%)$ & $245 \cdot 7(0 \cdot 40 \%)$ \\
\hline Chagas disease & $280 \cdot 1(0 \cdot 89 \%)$ & $383.9(0.62 \%)$ & $31.4(0.72 \%)$ & $42.9(0 \cdot 50 \%)$ & $95 \cdot 3(0 \cdot 15 \%)$ & $78 \cdot 3(0 \cdot 13 \%)$ & $210 \cdot 3(0 \cdot 34 \%)$ \\
\hline Endocarditis & $136 \cdot 2(0 \cdot 43 \%)$ & $250 \cdot 2(0 \cdot 41 \%)$ & $15 \cdot 7(0 \cdot 36 \%)$ & $29 \cdot 1(0 \cdot 34 \%)$ & $61 \cdot 6(0 \cdot 10 \%)$ & $50 \cdot 9(0 \cdot 08 \%)$ & $137 \cdot 6(0 \cdot 22 \%)$ \\
\hline $\begin{array}{l}\text { Other } \\
\text { haemoglobinopathies } \\
\text { and haemolytic } \\
\text { anaemias }\end{array}$ & $93 \cdot 0(0 \cdot 30 \%)$ & $216 \cdot 6(0 \cdot 35 \%)$ & $11 \cdot 0(0 \cdot 25 \%)$ & $25 \cdot 2(0 \cdot 29 \%)$ & $52 \cdot 9(0.09 \%)$ & $44 \cdot 3(0 \cdot 07 \%)$ & $119 \cdot 4(0 \cdot 19 \%)$ \\
\hline Thalassaemias & $96 \cdot 4(0 \cdot 31 \%)$ & $117 \cdot 8(0 \cdot 19 \%)$ & $11.9(0.27 \%)$ & $14.5(0 \cdot 17 \%)$ & $29 \cdot 3(0 \cdot 05 \%)$ & $23 \cdot 8(0 \cdot 04 \%)$ & $64 \cdot 7(0 \cdot 10 \%)$ \\
\hline $\begin{array}{l}\text { Interstitial lung disease } \\
\text { and pulmonary } \\
\text { sarcoidosis }\end{array}$ & $51 \cdot 3(0 \cdot 16 \%)$ & $102 \cdot 1(0 \cdot 17 \%)$ & $24 \cdot 3(0.56 \%)$ & $48 \cdot 1(0.56 \%)$ & $25 \cdot 2(0 \cdot 04 \%)$ & $20 \cdot 8(0.03 \%)$ & $56 \cdot 2(0 \cdot 09 \%)$ \\
\hline $\begin{array}{l}\text { Glucose-6-phosphate } \\
\text { dehydrogenase } \\
\text { deficiency }\end{array}$ & $8.1(0.03 \%)$ & $20.4(0.03 \%)$ & $1.0(0.02 \%)$ & $2.5(0.03 \%)$ & $5 \cdot 0(0.01 \%)$ & $4 \cdot 1(0.01 \%)$ & $11 \cdot 3(0 \cdot 02 \%)$ \\
\hline Silicosis & $10.8(0.03 \%)$ & $16 \cdot 2(0.03 \%)$ & $5 \cdot 2(0 \cdot 12 \%)$ & $7.8(0.09 \%)$ & $4.0(0.01 \%)$ & $3.3(0.01 \%)$ & $8.9(0.01 \%)$ \\
\hline $\begin{array}{l}\text { Coal workers' } \\
\text { pneumoconiosis }\end{array}$ & $5.8(0.02 \%)$ & $9 \cdot 0(0.01 \%)$ & $2.8(0.06 \%)$ & $4.3(0.05 \%)$ & $2 \cdot 2(0.00 \%)$ & $1.8(0.00 \%)$ & $5 \cdot 0(0 \cdot 01 \%)$ \\
\hline Iodine deficiency & $4.3(0.01 \%)$ & $6.9(0.01 \%)$ & $0.6(0.01 \%)$ & $0.9(0.01 \%)$ & $1.7(0.00 \%)$ & $1.4(0.00 \%)$ & $3 \cdot 8(0.01 \%)$ \\
\hline Asbestosis & $2.4(0.01 \%)$ & $4.1(0.01 \%)$ & $1 \cdot 1(0.03 \%)$ & $2.0(0.02 \%)$ & $1.0(0.00 \%)$ & $0.8(0.00 \%)$ & $2 \cdot 2(0.00 \%)$ \\
\hline Total prevalence & $31408 \cdot 5(100 \cdot 00 \%)$ & $61657 \cdot 6(100 \cdot 00 \%)$ & .. & .. & $15250 \cdot 2(24 \cdot 73 \%)$ & $12550 \cdot 3(20 \cdot 35 \%)$ & $33857 \cdot 1(54 \cdot 91 \%)$ \\
\hline Total YLDs & .. & .. & $4367.4(100 \cdot 00 \%)$ & $8558 \cdot 7(100 \cdot 00 \%)$ & $1069 \cdot 3(12.49 \%)$ & $1211 \cdot 1(14 \cdot 15 \%)$ & $6278.2(73.35 \%)$ \\
\hline
\end{tabular}

Data are number $(\%)$. YLDs=years lived with disability. 


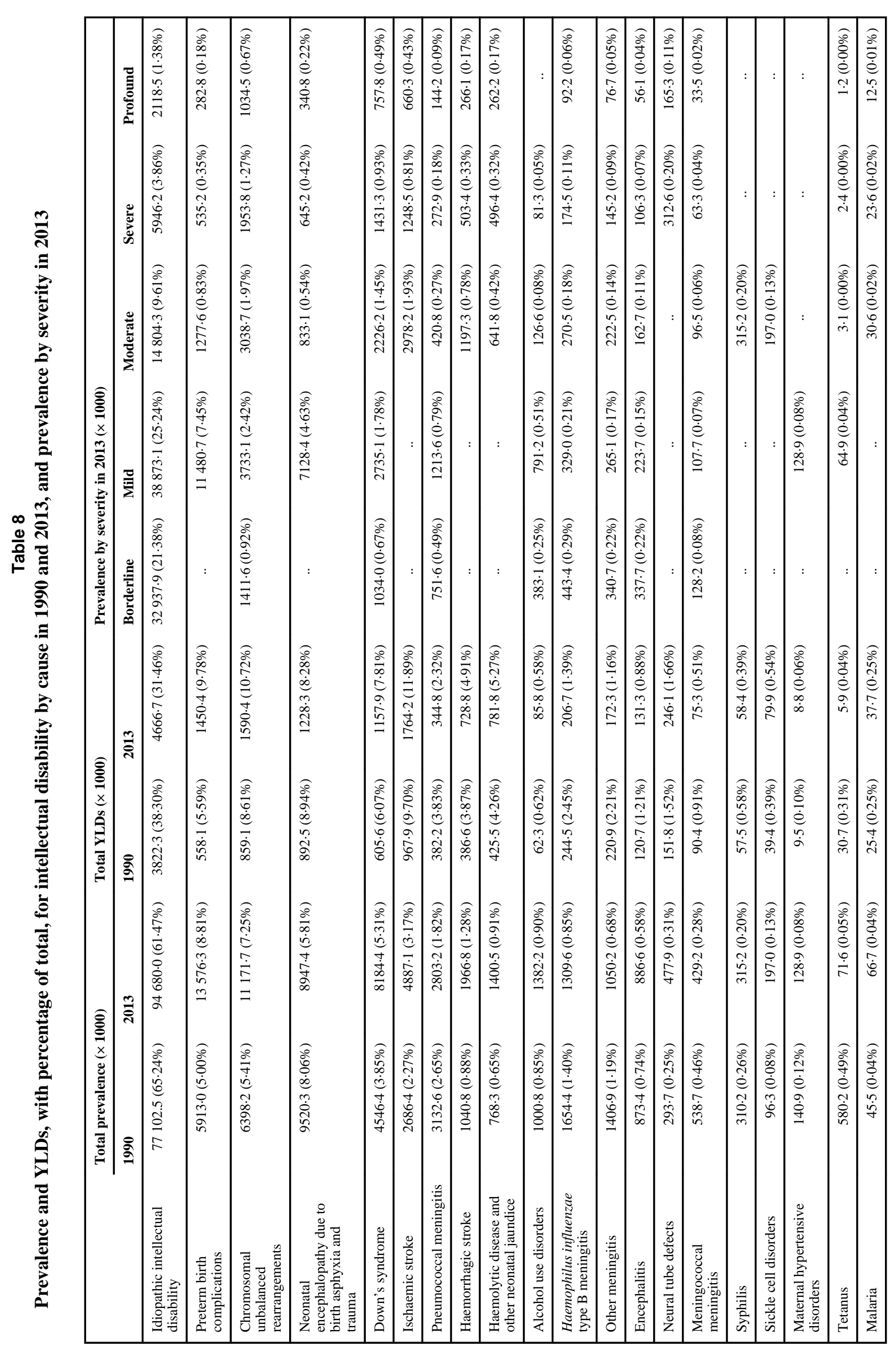


Page 82

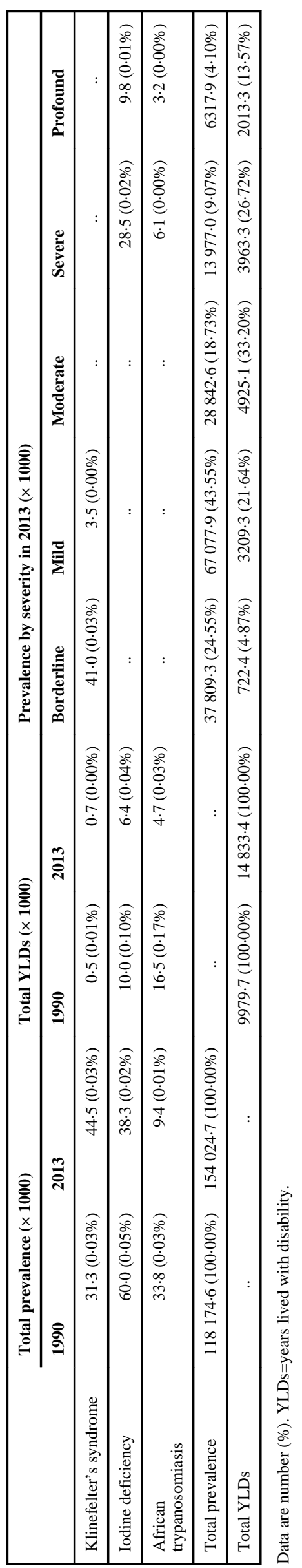


Table 9

Prevalence and YLDs, with percentage of total, for vision impairment by cause in 1990 and 2013, and prevalence by severity in 2013

\begin{tabular}{|c|c|c|c|c|c|c|c|c|}
\hline & \multicolumn{2}{|c|}{ Total prevalence $(\times 1000)$} & \multicolumn{2}{|l|}{ Total YLDs (×1000) } & \multicolumn{4}{|c|}{ Prevalence by severity in $2013(\times 1000)$} \\
\hline & 1990 & 2013 & 1990 & 2013 & Moderate & Severe & Blind & Presbyopia \\
\hline Uncorrected refractive error & $434222.6(83.74 \%)$ & $660118.8(85.28 \%)$ & $7831 \cdot 1(56 \cdot 60 \%)$ & $11257 \cdot 2(56 \cdot 00 \%)$ & $80451 \cdot 8(10 \cdot 39 \%)$ & $9943 \cdot 1(1.28 \%)$ & $9482.0(1.22 \%)$ & $560241.9(72 \cdot 38 \%)$ \\
\hline Cataract & $29915.0(5.77 \%)$ & $44223 \cdot 1(5 \cdot 71 \%)$ & $1860 \cdot 6(13 \cdot 45 \%)$ & $2916.7(14.51 \%)$ & $31636.5(4.09 \%)$ & $4857.8(0.63 \%)$ & $7728.8(1.00 \%)$ & .. \\
\hline Other vision loss & $22358 \cdot 1(4 \cdot 31 \%)$ & $26813.0(3.46 \%)$ & $1450 \cdot 4(10 \cdot 48 \%)$ & $1793.5(8.92 \%)$ & $19538.5(2.52 \%)$ & $2178 \cdot 7(0 \cdot 28 \%)$ & $5095 \cdot 8(0 \cdot 66 \%)$ & .. \\
\hline Macular degeneration & $7848.5(1.51 \%)$ & $13883.8(1.79 \%)$ & $412.9(2.98 \%)$ & $725.6(3.61 \%)$ & $11370 \cdot 5(1.47 \%)$ & $1498 \cdot 4(0 \cdot 19 \%)$ & $1014.9(0 \cdot 13 \%)$ & .. \\
\hline Glaucoma & $6692.0(1.29 \%)$ & $10900 \cdot 1(1 \cdot 41 \%)$ & $495 \cdot 7(3.58 \%)$ & $807.5(4.02 \%)$ & $7276 \cdot 1(0 \cdot 94 \%)$ & $984 \cdot 4(0.13 \%)$ & $2639 \cdot 6(0 \cdot 34 \%)$ & .. \\
\hline Preterm birth complications ${ }^{*}$ & $1844 \cdot 4(0 \cdot 36 \%)$ & $4412 \cdot 0(0.57 \%)$ & $434 \cdot 9(3 \cdot 14 \%)$ & $1135 \cdot 4(5 \cdot 65 \%)$ & $1022 \cdot 1(0 \cdot 13 \%)$ & $563.4(0.07 \%)$ & $2359.9(0 \cdot 30 \%)$ & .. \\
\hline Diabetes mellitus & $2631.4(0.51 \%)$ & $3816 \cdot 9(0.49 \%)$ & $325 \cdot 8(2 \cdot 35 \%)$ & $477.0(2.37 \%)$ & $1098 \cdot 8(0 \cdot 14 \%)$ & $163.7(0.02 \%)$ & $2554.5(0.33 \%)$ & .. \\
\hline Vitamin A deficiency & $4181.5(0.81 \%)$ & $3372 \cdot 2(0 \cdot 44 \%)$ & $199 \cdot 3(1.44 \%)$ & $153.7(0.76 \%)$ & $3014.5(0 \cdot 39 \%)$ & $203.5(0.03 \%)$ & $154 \cdot 2(0.02 \%)$ & .. \\
\hline Trachoma & $3983.4(0.77 \%)$ & $2428 \cdot 2(0 \cdot 31 \%)$ & $271.6(1.96 \%)$ & $171.2(0.85 \%)$ & $1659 \cdot 4(0 \cdot 21 \%)$ & $329 \cdot 8(0.04 \%)$ & $439.0(0.06 \%)$ & .. \\
\hline Onchocerciasis & $1907.5(0.37 \%)$ & $1183 \cdot 8(0 \cdot 15 \%)$ & $127.3(0.92 \%)$ & $78.4(0.39 \%)$ & $856 \cdot 5(0 \cdot 11 \%)$ & $142.5(0.02 \%)$ & $184 \cdot 8(0 \cdot 02 \%)$ & .. \\
\hline Pneumococcal meningitis $*$ & $950 \cdot 3(0 \cdot 18 \%)$ & $863.9(0.11 \%)$ & $42.5(0 \cdot 31 \%)$ & $35 \cdot 5(0 \cdot 18 \%)$ & .. & .. & $136 \cdot 9(0.02 \%)$ & .. \\
\hline $\begin{array}{l}\text { Neonatal encephalopathy due } \\
\text { to birth asphyxia and trauma }\end{array}$ & $293.6(0.06 \%)$ & $478.3(0.06 \%)$ & $158 \cdot 5(1 \cdot 15 \%)$ & $258.6(1.29 \%)$ & .. & .. & $478.3(0.06 \%)$ & .. \\
\hline Other meningitis $*$ & $522 \cdot 7(0 \cdot 10 \%)$ & $376 \cdot 1(0.05 \%)$ & $44 \cdot 2(0 \cdot 32 \%)$ & $30 \cdot 7(0 \cdot 15 \%)$ & .. & .. & $157.5(0.02 \%)$ & .. \\
\hline $\begin{array}{l}\text { Haemolytic disease and other } \\
\text { neonatal jaundice }\end{array}$ & $196.6(0.04 \%)$ & $373.9(0.05 \%)$ & $105 \cdot 6(0.76 \%)$ & $201.6(1.00 \%)$ & .. & .. & $373.9(0.05 \%)$ & .. \\
\hline $\begin{array}{l}\text { Haemophilus influenzae type } \\
\text { B meningitis } *\end{array}$ & $395.8(0.08 \%)$ & $298 \cdot 2(0.04 \%)$ & $9.7(0.07 \%)$ & $6.8(0.03 \%)$ & .. & .. & $11.9(0.00 \%)$ & .. \\
\hline Meningococcal meningitis ${ }^{*}$ & $358 \cdot 2(0.07 \%)$ & $270 \cdot 0(0.03 \%)$ & $46 \cdot 0(0.33 \%)$ & $34 \cdot 4(0 \cdot 17 \%)$ &. &. & $191 \cdot 3(0 \cdot 02 \%)$ &. \\
\hline Encephalitis ${ }^{*}$ & $247.5(0.05 \%)$ & $236.4(0.03 \%)$ & $9.3(0.07 \%)$ & $8 \cdot 1(0 \cdot 04 \%)$ & .. & .. & $25.5(0.00 \%)$ & .. \\
\hline Malaria & $11.7(0.00 \%)$ & $17.3(0.00 \%)$ & $6.2(0.04 \%)$ & $9 \cdot 2(0.05 \%)$ & .. & .. & $17.3(0.00 \%)$ & .. \\
\hline Tetanus & $6.3(0.00 \%)$ & $1.9(0.00 \%)$ & $3.4(0.02 \%)$ & $1.0(0.00 \%)$ & .. & .. & $1.9(0.00 \%)$ & .. \\
\hline Total prevalence & $518567 \cdot 1(100 \cdot 00 \%)$ & $774067 \cdot 8(100 \cdot 00 \%)$ & .. & .. & $157924 \cdot 6(20 \cdot 40 \%)$ & $20865 \cdot 2(2 \cdot 70 \%)$ & $33048 \cdot 1(4 \cdot 27 \%)$ & $560241.9(72.38 \%)$ \\
\hline Total YLDs & .. & .. & $13835 \cdot 0(100 \cdot 00 \%)$ & $20102 \cdot 2(100 \cdot 00 \%)$ & $4565.7(22.71 \%)$ & $3425 \cdot 7(17 \cdot 04 \%)$ & $6434.4(32 \cdot 01 \%)$ & $5650 \cdot 6(28 \cdot 11 \%)$ \\
\hline
\end{tabular}

Data are number $(\%)$. YLDs=years lived with disability.

$*$

Mild and monocular vision impairment not listed, but estimates are included in totals. 
Table 10

Prevalent cases and YLDs for 2013, percentage change, and percentage change of agestandardised rates between 1990 and 2013 for all causes

\begin{tabular}{|c|c|c|c|c|c|c|}
\hline & $\begin{array}{l}\text { Prevalent cases } \\
\text { in } 2013(\times \\
1000)\end{array}$ & $\begin{array}{l}\text { Percentage } \\
\text { change in } \\
\text { prevalence } \\
\text { from } 1990 \text { to } \\
2013\end{array}$ & $\begin{array}{l}\text { Percentage } \\
\text { change in age- } \\
\text { standardised } \\
\text { prevalence from } \\
1990 \text { to } 2013\end{array}$ & $\begin{array}{l}\text { YLDs in } \\
2013(x \\
1000)\end{array}$ & $\begin{array}{l}\text { Percentage } \\
\text { change in YLDs } \\
\text { from } 1990 \text { to } \\
2013\end{array}$ & $\begin{array}{l}\text { Percentage change } \\
\text { in age- } \\
\text { standardised } \\
\text { YLDs from } 1990 \\
\text { to } 2013\end{array}$ \\
\hline All causes &.. & .. & .. & $\begin{array}{r}764804 \cdot 4 \\
(572379 \cdot 6 \text { to } \\
990467 \cdot 0)\end{array}$ & $42 \cdot 3^{*}(40 \cdot 2$ to & $\begin{array}{r}-3 \cdot 9 *(-5 \cdot 1 \text { to } \\
-3 \cdot 0)\end{array}$ \\
\hline $\begin{array}{l}\text { Communicable, maternal, neonatal, } \\
\text { and nutritional diseases }\end{array}$ &.. &. & .. & $\begin{array}{r}101495 \cdot 1 \\
(71533 \cdot 1 \text { to } \\
138458 \cdot 0)\end{array}$ & $7 \cdot 6^{*}(4 \cdot 0$ to $11 \cdot 8)$ & 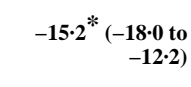 \\
\hline HIV/AIDS and tuberculosis & .. & .. & .. & $\begin{array}{r}7733.3 \\
(5494.4 \text { to } 10 \\
077.9)\end{array}$ & $\begin{array}{r}125 \cdot 4^{*}(113.7 \text { to } \\
141 \cdot 0)\end{array}$ & $47 \cdot 1^{*}$ (39.5 to $\left.57 \cdot 4\right)$ \\
\hline Tuberculosis & $\begin{array}{r}12111.8(11 \\
791.6 \text { to } 12 \\
452 \cdot 0)\end{array}$ & $\begin{array}{r}51 \cdot 6^{*}(49 \cdot 3 \text { to } \\
54 \cdot 0)\end{array}$ & $\begin{array}{r}-1.9 *(-3.3 \text { to } \\
-0 \cdot 3)\end{array}$ & $\begin{array}{r}3669 \cdot 7 \\
(2519 \cdot 7 \text { to } \\
4889 \cdot 1)\end{array}$ & $\begin{array}{r}51.9 *(48 \cdot 6 \text { to } \\
55 \cdot 2)\end{array}$ & $-1 \cdot 3(-3 \cdot 4$ to $0 \cdot 7)$ \\
\hline HIV/AIDS &.. &.. & .. & $\begin{array}{r}4063 \cdot 7 \\
(2918 \cdot 9 \text { to } \\
5327 \cdot 4)\end{array}$ & $\begin{array}{r}301.7 *(259 \cdot 4 \text { to } \\
354 \cdot 3)\end{array}$ & $\begin{array}{r}171.5 *(142.0 \text { to } \\
208 \cdot 7)\end{array}$ \\
\hline $\begin{array}{l}\text { HIV/AIDS resulting in } \\
\text { mycobacterial infection }\end{array}$ & $\begin{array}{r}724 \cdot 7(496 \cdot 0 \text { to } \\
922 \cdot 5)\end{array}$ & 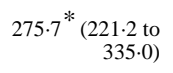 & $\begin{array}{r}144 \cdot 0^{*}(107 \cdot 3 \text { to } \\
184 \cdot 1)\end{array}$ & $\begin{array}{r}268 \cdot 3(162 \cdot 5 \\
\text { to } 388 \cdot 6)\end{array}$ & 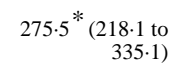 & $\begin{array}{r}144.6 *(104.9 \text { to } \\
185.4)\end{array}$ \\
\hline $\begin{array}{l}\text { HIV/AIDS resulting in other } \\
\text { diseases }\end{array}$ & $\begin{array}{r}28506 \cdot 6(27 \\
415 \cdot 1 \text { to } 30 \\
970 \cdot 4)\end{array}$ & $\begin{array}{r}234 \cdot 1^{*}(217 \cdot 8 \text { to } \\
262 \cdot 8)\end{array}$ & $\begin{array}{r}134.5^{*}(122 \cdot 8 \text { to } \\
154 \cdot 8)\end{array}$ & $\begin{array}{r}3795 \cdot 3 \\
(2726 \cdot 7 \text { to } \\
4974 \cdot 6)\end{array}$ & $\begin{array}{r}304 \cdot 1^{*}(257.2 \text { to } \\
361 \cdot 0)\end{array}$ & $\begin{array}{r}174 \cdot 0 *(140 \cdot 9 \text { to } \\
214 \cdot 3)\end{array}$ \\
\hline $\begin{array}{l}\text { Diarrhoea, lower respiratory, and } \\
\text { other common infectious diseases }\end{array}$ &.$\cdot$ & .. & .. & $\begin{array}{r}14339 \cdot 7(10 \\
108 \cdot 4 \text { to } 19 \\
541 \cdot 9)\end{array}$ & $0 \cdot 2(-2 \cdot 8$ to $4 \cdot 6)$ & $\begin{array}{r}-18 \cdot 5^{*}(-18 \cdot 5 \text { to } \\
-13 \cdot 6)\end{array}$ \\
\hline Diarrhoeal diseases & $\begin{array}{r}42409 \cdot 6(41 \\
769 \cdot 5 \text { to } 43 \\
170 \cdot 2)\end{array}$ & $\begin{array}{r}-6.9 *(-8.9 \text { to } \\
-4.7)\end{array}$ & $\begin{array}{r}-18 \cdot 3^{*}(-20 \cdot 1 \text { to } \\
-16 \cdot 4)\end{array}$ & $\begin{array}{r}6854 \cdot 2 \\
(4701 \cdot 9 \text { to } \\
9415 \cdot 3)\end{array}$ & $\begin{array}{r}-6.9 *(-9 \cdot 2 \text { to } \\
-4.7)\end{array}$ & $\begin{array}{r}-18.0 * \text { (-19.9 to } \\
-16 \cdot 0)\end{array}$ \\
\hline Intestinal infectious diseases & .. & .. & .. & $\begin{array}{r}203 \cdot 7(138 \cdot 5 \\
\text { to } 281 \cdot 4)\end{array}$ & $\begin{array}{r}-23 \cdot 3^{*}(-32 \cdot 2 \text { to } \\
-12 \cdot 9)\end{array}$ & $\begin{array}{r}-35 \cdot 7^{*}(-43 \cdot 1 \text { to } \\
-27 \cdot 3)\end{array}$ \\
\hline Typhoid fever & $\begin{array}{r}1198 \cdot 3(1103 \cdot 3 \\
\text { to } 1300 \cdot 6)\end{array}$ & $\begin{array}{r}-19 \cdot 9 *(-30 \cdot 7 \\
\text { to }-6 \cdot 5)\end{array}$ & $\begin{array}{r}-33.3^{*}(-41.9 \text { to } \\
-22 \cdot 4)\end{array}$ & $\begin{array}{r}158 \cdot 6(109 \cdot 0 \\
\text { to } 223 \cdot 3)\end{array}$ & $\begin{array}{r}-19.0 *(-30 \cdot 5 \text { to } \\
-4.2)\end{array}$ & $\begin{array}{r}-32.3^{*}(-41.8 \text { to } \\
-20.5)\end{array}$ \\
\hline Paratyphoid fever & $\begin{array}{r}735.9(640 \cdot 4 \text { to } \\
836 \cdot 9)\end{array}$ & $\begin{array}{r}-27.9 *(-41.8 \\
\text { to }-12 \cdot 8)\end{array}$ & $\begin{array}{r}-39 \cdot 1^{*}(-50 \cdot 9 \text { to } \\
-26 \cdot 5)\end{array}$ & $\begin{array}{r}38 \cdot 3(24.0 \text { to } \\
57 \cdot 6)\end{array}$ & $\begin{array}{r}-27.1^{*}(-41 \cdot 6 \text { to } \\
-9 \cdot 7)\end{array}$ & $\begin{array}{r}-38 \cdot 4^{*}(-50 \cdot 3 \text { to } \\
-23 \cdot 9)\end{array}$ \\
\hline $\begin{array}{l}\text { Other intestinal infectious } \\
\text { diseases }\end{array}$ &.. & .. & .. & $\begin{array}{r}6 \cdot 8(3 \cdot 2 \text { to } \\
13 \cdot 4)\end{array}$ & $\begin{array}{r}-61.0 *(-67.6 \text { to } \\
-51.8)\end{array}$ & $\begin{array}{r}-67.4 *(-72.9 \text { to } \\
-59.7)\end{array}$ \\
\hline Lower respiratory infections & $\begin{array}{r}4473 \cdot 3(4374 \cdot 7 \\
\text { to } 4554 \cdot 2)\end{array}$ & $\begin{array}{r}-12 \cdot 8 *(-15 \cdot 7 \\
\text { to }-10 \cdot 7)\end{array}$ & $\begin{array}{r}-25.5^{*}(-27.9 \text { to } \\
-23 \cdot 8)\end{array}$ & $\begin{array}{r}460 \cdot 9(310 \cdot 5 \\
\text { to } 642 \cdot 7)\end{array}$ & $\begin{array}{r}-14.1^{*}(-17.4 \text { to } \\
-11.7)\end{array}$ & $\begin{array}{r}-26 \cdot 1^{*}(-28 \cdot 9 \text { to } \\
-24 \cdot 2)\end{array}$ \\
\hline Upper respiratory infections & $\begin{array}{r}244327 \cdot 5(240 \\
789 \cdot 6 \text { to } 247 \\
629 \cdot 2)\end{array}$ & $\begin{array}{r}38.4 *(35.7 \text { to } \\
41.5)\end{array}$ & $6 \cdot 3^{*}(4 \cdot 2$ to $8 \cdot 6)$ & $\begin{array}{r}2863 \cdot 1 \\
(1597 \cdot 9 \text { to } \\
4763 \cdot 5)\end{array}$ & $\begin{array}{r}38.4 *(35.4 \text { to } \\
41.6)\end{array}$ & $6.6^{*}(4.4$ to 9.0$)$ \\
\hline Otitis media & $\begin{array}{r}85228 \cdot 0(82 \\
190 \cdot 3 \text { to } 88 \\
667 \cdot 4)\end{array}$ & $\begin{array}{r}14.4^{*}(11.9 \text { to } \\
16.9)\end{array}$ & $\begin{array}{r}-11 \cdot 1^{*}(-12 \cdot 9 \text { to } \\
-9 \cdot 1)\end{array}$ & $\begin{array}{r}1696 \cdot 4 \\
(1017 \cdot 6 \text { to } \\
2697.9)\end{array}$ & $\begin{array}{r}14.4 *(11.4 \text { to } \\
17 \cdot 6)\end{array}$ & $\begin{array}{r}-10 \cdot 1^{*}(-12 \cdot 2 \text { to } \\
-7 \cdot 7)\end{array}$ \\
\hline Meningitis &.. & .. & .. & $\begin{array}{r}1679 \cdot 1 \\
(1165 \cdot 8 \text { to } \\
2259 \cdot 6)\end{array}$ & $\begin{array}{r}-11.7^{*}(-15.5 \text { to } \\
-7.9)\end{array}$ & $\begin{array}{r}-31.4 *(-34.4 \text { to } \\
-27 \cdot 8)\end{array}$ \\
\hline Pneumococcal & $\begin{array}{r}7805 \cdot 6(4929.9 \\
\text { to } 11605 \cdot 5)\end{array}$ & $\begin{array}{r}-5 \cdot 5(-11 \cdot 3 \text { to } \\
1 \cdot 8)\end{array}$ & $\begin{array}{r}-30 \cdot 7^{*}(-34 \cdot 7 \text { to } \\
-25 \cdot 6)\end{array}$ & $\begin{array}{r}698 \cdot 1(494 \cdot 1 \\
\text { to } 917 \cdot 8)\end{array}$ & $\begin{array}{r}-5 \cdot 2(-11 \cdot 5 \text { to } \\
3 \cdot 4)\end{array}$ & $\begin{array}{r}-27.6^{*}(-32 \cdot 1 \text { to } \\
-20 \cdot 9)\end{array}$ \\
\hline Haemophilus influenzae type B & $\begin{array}{r}3246 \cdot 2(1373 \cdot 4 \\
\text { to } 5962 \cdot 2)\end{array}$ & $\begin{array}{r}-19 \cdot 1 *(-26 \cdot 4 \\
\text { to }-12 \cdot 0)\end{array}$ & $\begin{array}{r}-38 \cdot 5^{*}(-43 \cdot 6 \text { to } \\
-32 \cdot 2)\end{array}$ & $\begin{array}{r}375 \cdot 5(258 \cdot 8 \\
\text { to } 510 \cdot 3)\end{array}$ & $\begin{array}{r}-13 \cdot 7^{*}(-20 \cdot 5 \text { to } \\
-3 \cdot 3)\end{array}$ & $\begin{array}{r}-31 \cdot 0 *(-36 \cdot 3 \text { to } \\
-22 \cdot 3)\end{array}$ \\
\hline
\end{tabular}




\begin{tabular}{|c|c|c|c|c|c|c|}
\hline & $\begin{array}{l}\text { Prevalent cases } \\
\text { in 2013 }(\times \\
1000)\end{array}$ & $\begin{array}{l}\text { Percentage } \\
\text { change in } \\
\text { prevalence } \\
\text { from } 1990 \text { to } \\
2013\end{array}$ & $\begin{array}{l}\text { Percentage } \\
\text { change in age- } \\
\text { standardised } \\
\text { prevalence from } \\
1990 \text { to } 2013\end{array}$ & $\begin{array}{l}\text { YLDs in } \\
2013(x \\
1000)\end{array}$ & $\begin{array}{l}\text { Percentage } \\
\text { change in YLDs } \\
\text { from } 1990 \text { to } \\
2013\end{array}$ & $\begin{array}{l}\text { Percentage change } \\
\text { in age- } \\
\text { standardised } \\
\text { YLDs from } 1990 \\
\text { to } 2013\end{array}$ \\
\hline Meningococcal & $\begin{array}{r}1321.2(490 \cdot 6 \\
\text { to } 2847.6)\end{array}$ & $\begin{array}{r}-19.9 *(-28.5 \\
\text { to }-12 \cdot 0)\end{array}$ & $\begin{array}{r}-40 \cdot 9 *(-46 \cdot 6 \text { to } \\
-34 \cdot 5)\end{array}$ & $\begin{array}{r}167 \cdot 3(106 \cdot 3 \\
\text { to } 251 \cdot 6)\end{array}$ & $\begin{array}{r}-17 \cdot 1^{*}(-25 \cdot 0 \text { to } \\
-8 \cdot 0)\end{array}$ & $\begin{array}{r}-37.2 *(-42.5 \text { to } \\
-29.3)\end{array}$ \\
\hline Other meningitis & $\begin{array}{r}3595 \cdot 2(1901.7 \\
\text { to } 6305 \cdot 8)\end{array}$ & $\begin{array}{r}-20.2 *(-27.9 \\
\text { to }-12.9)\end{array}$ & $\begin{array}{r}-39 \cdot 6 *(-44 \cdot 9 \text { to } \\
-33 \cdot 2)\end{array}$ & $\begin{array}{r}438 \cdot 3(299 \cdot 2 \\
\text { to } 604 \cdot 2)\end{array}$ & $\begin{array}{r}-17 \cdot 4^{*}(-23 \cdot 3 \text { to } \\
-11.9)\end{array}$ & $\begin{array}{r}-35.2 *(-39.7 \text { to } \\
-30 \cdot 7)\end{array}$ \\
\hline Encephalitis & $\begin{array}{r}1739 \cdot 8(795 \cdot 7 \\
\text { to } 4003 \cdot 7)\end{array}$ & $\begin{array}{r}3 \cdot 1(-16 \cdot 5 \text { to } \\
12 \cdot 6)\end{array}$ & $\begin{array}{r}-27 \cdot 5^{*}(-41 \cdot 1 \text { to } \\
-21 \cdot 1)\end{array}$ & $\begin{array}{r}229 \cdot 1(159 \cdot 9 \\
\text { to } 305 \cdot 7)\end{array}$ & $\begin{array}{r}10 \cdot 8 * \text { (4.7 to } \\
17.9)\end{array}$ & $\begin{array}{r}-20 \cdot 6^{*}(-25 \cdot 0 \text { to } \\
-15 \cdot 4)\end{array}$ \\
\hline Diphtheria & $1.1(0.7$ to 2.0$)$ & $\begin{array}{r}-62 \cdot 1 *(-83 \cdot 4 \\
\text { to }-15 \cdot 4)\end{array}$ & $\begin{array}{r}-65 \cdot 7^{*}(-84 \cdot 6 \text { to } \\
-25 \cdot 0)\end{array}$ & $\begin{array}{r}0.1(0 \cdot 0 \text { to } \\
0 \cdot 2)\end{array}$ & $\begin{array}{r}-62 \cdot 1^{*}(-83 \cdot 5 \text { to } \\
-14.8)\end{array}$ & $\begin{array}{r}-65 \cdot 8^{*}(-84.6 \text { to } \\
-25 \cdot 0)\end{array}$ \\
\hline Whooping cough & $\begin{array}{r}2532 \cdot 5(1965 \cdot 7 \\
\text { to } 3239 \cdot 2)\end{array}$ & $\begin{array}{r}-30 \cdot 0 *(-30 \cdot 6 \\
\text { to }-29 \cdot 4)\end{array}$ & $\begin{array}{r}-32.5 *(-33 \cdot 1 \text { to } \\
-31 \cdot 8)\end{array}$ & $\begin{array}{r}125 \cdot 5(73 \cdot 0 \\
\text { to } 200 \cdot 7)\end{array}$ & $\begin{array}{r}-30.0 *(-31.9 \text { to } \\
-27.7)\end{array}$ & $\begin{array}{r}-32 \cdot 4^{*}(-34 \cdot 3 \text { to } \\
-30 \cdot 2)\end{array}$ \\
\hline Tetanus & $\begin{array}{r}177 \cdot 1(117 \cdot 5 \text { to } \\
265 \cdot 7)\end{array}$ & $\begin{array}{r}-87 \cdot 1 *(-92.5 \\
\text { to }-77 \cdot 9)\end{array}$ & $\begin{array}{r}-90 \cdot 1 *(-94 \cdot 2 \text { to } \\
-82 \cdot 9)\end{array}$ & $\begin{array}{r}13 \cdot 2(8 \cdot 5 \text { to } \\
21 \cdot 3)\end{array}$ & $\begin{array}{r}-78 \cdot 2^{*}(-85 \cdot 4 \text { to } \\
-64 \cdot 5)\end{array}$ & $\begin{array}{r}-82.2 *(-88.2 \text { to } \\
-71 \cdot 0)\end{array}$ \\
\hline Measles & $\begin{array}{r}192.4(150 \cdot 4 \text { to } \\
242 \cdot 9)\end{array}$ & $\begin{array}{r}-77 \cdot 1{ }^{*}(-79 \cdot 2 \\
\text { to }-74 \cdot 9)\end{array}$ & $\begin{array}{r}-78.0 *(-80 \cdot 1 \text { to } \\
-75.9)\end{array}$ & $\begin{array}{r}17 \cdot 3(10 \cdot 2 \text { to } \\
26 \cdot 7)\end{array}$ & $\begin{array}{r}-77 \cdot 1 *(-80 \cdot 0 \text { to } \\
-73 \cdot 8)\end{array}$ & $\begin{array}{r}-78.0 *(-80.8 \text { to } \\
-74.8)\end{array}$ \\
\hline Varicella and herpes zoster & $\begin{array}{r}5715 \cdot 8(5587 \cdot 6 \\
\text { to } 5835 \cdot 3)\end{array}$ & $\begin{array}{r}28.4 *(25.5 \text { to } \\
31.7)\end{array}$ & $-2.4(-4.9$ to 0.3$)$ & $\begin{array}{r}197 \cdot 2(122 \cdot 1 \\
\text { to } 299 \cdot 2)\end{array}$ & $\begin{array}{r}47 \cdot 6^{*}(41 \cdot 1 \text { to } \\
54 \cdot 5)\end{array}$ & $-1 \cdot 8(-5 \cdot 7$ to $2 \cdot 7)$ \\
\hline $\begin{array}{l}\text { Neglected tropical diseases and } \\
\text { malaria }\end{array}$ & .. & .. & .. & $\begin{array}{r}20100 \cdot 7(13 \\
202 \cdot 4 \text { to } 28 \\
946 \cdot 1)\end{array}$ & $\begin{array}{r}-3 \cdot 3(-10 \cdot 2 \text { to } \\
6 \cdot 7)\end{array}$ & $\begin{array}{r}-28 \cdot 7^{*}(-33 \cdot 1 \text { to } \\
-23 \cdot 0)\end{array}$ \\
\hline Malaria & $\begin{array}{r}351051 \cdot 1(344 \\
772 \cdot 1 \text { to } 358 \\
013 \cdot 6)\end{array}$ & $\begin{array}{r}30.8^{*}(28 \cdot 4 \text { to } \\
33 \cdot 2)\end{array}$ & $3.0 *(1 \cdot 1$ to 4.9$)$ & $\begin{array}{r}3170 \cdot 5 \\
(2132 \cdot 3 \text { to } \\
4591 \cdot 9)\end{array}$ & $\begin{array}{r}30 \cdot 1 *(25 \cdot 2 \text { to } \\
35 \cdot 0)\end{array}$ & $7.8^{*}(3.7$ to 11.9$)$ \\
\hline Chagas disease & $\begin{array}{r}9433 \cdot 9(9241 \cdot 1 \\
\text { to } 9628 \cdot 4)\end{array}$ & $\begin{array}{r}22.4 *(19.5 \text { to } \\
25 \cdot 7)\end{array}$ & $\begin{array}{r}-16 \cdot 3 *(-18 \cdot 3 \text { to } \\
-14 \cdot 0)\end{array}$ & $\begin{array}{r}97.5(64.9 \text { to } \\
137.0)\end{array}$ & $33 \cdot 6 * \begin{array}{r}(28 \cdot 8 \text { to } \\
39 \cdot 1)\end{array}$ & $\begin{array}{r}-17 \cdot 4^{*}(-20 \cdot 2 \text { to } \\
-14 \cdot 2)\end{array}$ \\
\hline Leishmaniasis &.. & .. & .. & $\begin{array}{r}49 \cdot 7(25 \cdot 5 \text { to } \\
90 \cdot 4)\end{array}$ & $\begin{array}{r}134 \cdot 7^{*}(107 \cdot 8 \text { to } \\
165 \cdot 0)\end{array}$ & $75.8^{*}(58.0$ to $97 \cdot 3)$ \\
\hline Visceral & $\begin{array}{r}113 \cdot 7(94 \cdot 1 \text { to } \\
140 \cdot 9)\end{array}$ & $\begin{array}{r}35 \cdot 1^{*}(17 \cdot 1 \text { to } \\
54 \cdot 9)\end{array}$ & $14 \cdot 4(-0 \cdot 4$ to $30 \cdot 5)$ & $\begin{array}{r}8 \cdot 0(5 \cdot 2 \text { to } \\
12 \cdot 3)\end{array}$ & $\begin{array}{r}35.8 *(14.6 \text { to } \\
61.8)\end{array}$ & $15 \cdot 2(-2 \cdot 5$ to $36 \cdot 7)$ \\
\hline Cutaneous and mucocutaneous & $\begin{array}{r}3914 \cdot 8(3300 \cdot 4 \\
\text { to } 4669 \cdot 6)\end{array}$ & $\begin{array}{r}174 \cdot 2^{*}(144 \cdot 5 \text { to } \\
209 \cdot 2)\end{array}$ & $\begin{array}{r}95 \cdot 3^{*}(75 \cdot 2 \text { to } \\
119 \cdot 9)\end{array}$ & $\begin{array}{r}41.7(19.0 \text { to } \\
80 \cdot 1)\end{array}$ & $\begin{array}{r}175 \cdot 1 *(146 \cdot 0 \text { to } \\
210 \cdot 9)\end{array}$ & $\begin{array}{r}97 \cdot 1 *(76 \cdot 6 \text { to } \\
122 \cdot 4)\end{array}$ \\
\hline African trypanosomiasis & $\begin{array}{r}19 \cdot 7(10 \cdot 6 \text { to } \\
34 \cdot 3)\end{array}$ & $\begin{array}{r}-71 \cdot 1 *(-75 \cdot 3 \\
\text { to }-65 \cdot 9)\end{array}$ & $\begin{array}{r}-79 \cdot 5 *(-82 \cdot 4 \text { to } \\
-75 \cdot 8)\end{array}$ & $\begin{array}{r}5.4(2 \cdot 6 \text { to } \\
9 \cdot 5)\end{array}$ & $\begin{array}{r}-70 \cdot 7^{*}(-75 \cdot 3 \text { to } \\
-64 \cdot 5)\end{array}$ & $\begin{array}{r}-79 \cdot 1 *(-82 \cdot 3 \text { to } \\
-74 \cdot 7)\end{array}$ \\
\hline Schistosomiasis & $\begin{array}{r}290627 \cdot 9(252 \\
098 \cdot 7 \text { to } 337 \\
576 \cdot 1)\end{array}$ & $\begin{array}{r}30.9^{*}(22.9 \text { to } \\
51.9)\end{array}$ & $-3 \cdot 7(-9.7$ to $11 \cdot 8)$ & $\begin{array}{r}2861 \cdot 7 \\
(1483 \cdot 6 \text { to } \\
5467 \cdot 2)\end{array}$ & $34 \cdot 1 * \begin{array}{r}(26 \cdot 4 \text { to } \\
55 \cdot 4)\end{array}$ & $0 \cdot 3(-5 \cdot 6$ to $16 \cdot 3)$ \\
\hline Cysticercosis & $\begin{array}{r}1030 \cdot 8(901.4 \\
\text { to } 1185 \cdot 6)\end{array}$ & $\begin{array}{r}-26 \cdot 3 *(-37 \cdot 3 \\
\text { to }-10 \cdot 0)\end{array}$ & $\begin{array}{r}-50 \cdot 2^{*}(-57.5 \text { to } \\
-39 \cdot 0)\end{array}$ & $\begin{array}{r}310 \cdot 4(212 \cdot 2 \\
\text { to } 409 \cdot 5)\end{array}$ & $\begin{array}{r}-20.9^{*}(-33 \cdot 9 \text { to } \\
-3 \cdot 0)\end{array}$ & $\begin{array}{r}-46 \cdot 5^{*}(-55 \cdot 1 \text { to } \\
-34 \cdot 4)\end{array}$ \\
\hline Cystic echinococcosis & $\begin{array}{r}849 \cdot 2(822 \cdot 5 \text { to } \\
893 \cdot 2)\end{array}$ & $\begin{array}{r}-15 \cdot 4{ }^{*}(-17 \cdot 0 \\
\text { to }-12 \cdot 9)\end{array}$ & $\begin{array}{r}-42.4^{*}(-43.5 \text { to } \\
-40 \cdot 8)\end{array}$ & $\begin{array}{r}79 \cdot 2(54.9 \text { to } \\
110 \cdot 3)\end{array}$ & $\begin{array}{r}-15 \cdot 3 *(-18 \cdot 9 \text { to } \\
-11 \cdot 0)\end{array}$ & $\begin{array}{r}-42.0 *(-44.3 \text { to } \\
-39 \cdot 2)\end{array}$ \\
\hline Lymphatic filariasis & $\begin{array}{r}43850 \cdot 0(36 \\
940 \cdot 8 \text { to } 52 \\
905 \cdot 8)\end{array}$ & $\begin{array}{r}-32 \cdot 1 *(-39 \cdot 1 \\
\text { to }-24 \cdot 7)\end{array}$ & $\begin{array}{r}-53 \cdot 3^{*}(-58 \cdot 0 \text { to } \\
-48 \cdot 4)\end{array}$ & $\begin{array}{r}2022 \cdot 1 \\
(1096 \cdot 3 \text { to } \\
3294 \cdot 4)\end{array}$ & $\begin{array}{r}5 \cdot 9(-15 \cdot 7 \text { to } \\
26 \cdot 4)\end{array}$ & $\begin{array}{r}-32.3^{*}(-46 \cdot 0 \text { to } \\
-19 \cdot 3)\end{array}$ \\
\hline Onchocerciasis & $\begin{array}{r}16956 \cdot 4(11 \\
477 \cdot 5 \text { to } 26 \\
789 \cdot 4)\end{array}$ & $\begin{array}{r}-31.2^{*}(-39 \cdot 8 \\
\text { to }-21.9)\end{array}$ & $\begin{array}{r}-51.5^{*}(-57 \cdot 6 \text { to } \\
-45 \cdot 3)\end{array}$ & $\begin{array}{r}1179 \cdot 8 \\
(556 \cdot 6 \text { to } \\
1992 \cdot 7)\end{array}$ & $\begin{array}{r}-25 \cdot 4^{*}(-37 \cdot 0 \text { to } \\
-10 \cdot 9)\end{array}$ & $\begin{array}{r}-48.9^{*}(-57.7 \text { to } \\
-38.4)\end{array}$ \\
\hline Trachoma & $\begin{array}{r}2428 \cdot 8(1924.2 \\
\text { to } 2981 \cdot 2)\end{array}$ & $\begin{array}{r}-39 \cdot 2 *(-46 \cdot 3 \\
\text { to }-30 \cdot 1)\end{array}$ & $\begin{array}{r}-65 \cdot 4^{*}(-69.5 \text { to } \\
-60 \cdot 3)\end{array}$ & $\begin{array}{r}171 \cdot 2(115 \cdot 3 \\
\text { to } 241 \cdot 7)\end{array}$ & $\begin{array}{r}-37 \cdot 2^{*}(-43 \cdot 0 \text { to } \\
-30 \cdot 4)\end{array}$ & $\begin{array}{r}-64.2^{*}(-67.5 \text { to } \\
-60.5)\end{array}$ \\
\hline Dengue & $\begin{array}{r}3485 \cdot 5(1283 \cdot 7 \\
\text { to } 7818 \cdot 6)\end{array}$ & $\begin{array}{r}610 \cdot 9 *(606 \cdot 3 \text { to } \\
615 \cdot 5)\end{array}$ & $\begin{array}{r}447 \cdot 3^{*}(443 \cdot 6 \text { to } \\
450 \cdot 9)\end{array}$ & $\begin{array}{r}565.9(186 \cdot 4 \\
\text { to } 1414 \cdot 6)\end{array}$ & $\begin{array}{r}607.2 *(558.4 \text { to } \\
659.5)\end{array}$ & $\begin{array}{r}446.8 *(411.3 \text { to } \\
486 \cdot 8)\end{array}$ \\
\hline Yellow fever & $2 \cdot 1(0 \cdot 7$ to 4.9$)$ & $\begin{array}{r}-59.8 *(-63.7 \\
\text { to }-54 \cdot 3)\end{array}$ & $\begin{array}{r}-68 \cdot 3^{*}(-71 \cdot 4 \text { to } \\
-64 \cdot 0)\end{array}$ & $\begin{array}{r}0 \cdot 1(0 \cdot 0 \text { to } \\
0 \cdot 2)\end{array}$ & $\begin{array}{r}-59.8^{*}(-63.7 \text { to } \\
-54 \cdot 3)\end{array}$ & $\begin{array}{r}-68 \cdot 3^{*}(-71 \cdot 4 \text { to } \\
-64 \cdot 0)\end{array}$ \\
\hline
\end{tabular}




\begin{tabular}{|c|c|c|c|c|c|c|}
\hline & $\begin{array}{l}\text { Prevalent cases } \\
\text { in } 2013(\times \\
1000)\end{array}$ & $\begin{array}{l}\text { Percentage } \\
\text { change in } \\
\text { prevalence } \\
\text { from } 1990 \text { to } \\
2013\end{array}$ & $\begin{array}{l}\text { Percentage } \\
\text { change in age- } \\
\text { standardised } \\
\text { prevalence from } \\
1990 \text { to } 2013\end{array}$ & $\begin{array}{l}\text { YLDs in } \\
2013(x \\
1000)\end{array}$ & $\begin{array}{l}\text { Percentage } \\
\text { change in YLDs } \\
\text { from } 1990 \text { to } \\
2013\end{array}$ & $\begin{array}{l}\text { Percentage change } \\
\text { in age- } \\
\text { standardised } \\
\text { YLDs from } 1990 \\
\text { to } 2013\end{array}$ \\
\hline Rabies & $0.9(0.7$ to 1.1$)$ & $\begin{array}{r}-39 \cdot 8 *(-54 \cdot 3 \\
\text { to }-27 \cdot 3)\end{array}$ & $\begin{array}{r}-55 \cdot 2 *(-65 \cdot 1 \text { to } \\
-46 \cdot 1)\end{array}$ & $\begin{array}{r}0.1(0 \cdot 1 \text { to } \\
0 \cdot 2)\end{array}$ & $\begin{array}{r}-39 \cdot 8^{*}(-54 \cdot 3 \text { to } \\
-27 \cdot 2)\end{array}$ & 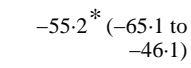 \\
\hline Intestinal nematode infections & .. & .. & .. & $\begin{array}{r}3691.6 \\
(2185.8 \text { to } \\
5801.7)\end{array}$ & $\begin{array}{r}-46 \cdot 0 *(-50 \cdot 6 \text { to } \\
-41 \cdot 4)\end{array}$ & $\begin{array}{r}-58 \cdot 8^{*}(-62 \cdot 3 \text { to } \\
-55 \cdot 3)\end{array}$ \\
\hline Ascariasis & $\begin{array}{r}804370 \cdot 1(713 \\
417.5 \text { to } 922 \\
212 \cdot 1)\end{array}$ & $\begin{array}{r}-25.5 *(-37.8 \\
\text { to }-10.4)\end{array}$ & 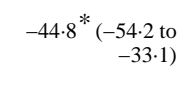 & $\begin{array}{r}933.9(516 \cdot 6 \\
\text { to } 1576 \cdot 1)\end{array}$ & $\begin{array}{r}-74.2^{*}(-77 \cdot 4 \text { to } \\
-70 \cdot 6)\end{array}$ & $\begin{array}{r}-80 \cdot 1^{*}(-82.5 \text { to } \\
-77 \cdot 3)\end{array}$ \\
\hline Trichuriasis & $\begin{array}{r}477374 \cdot 4(441 \\
256 \cdot 8 \text { to } 518 \\
365 \cdot 1)\end{array}$ & $\begin{array}{r}-11.6(-28.7 \text { to } \\
4.9)\end{array}$ & $\begin{array}{r}-34.4 *(-47.8 \text { to } \\
-21 \cdot 0)\end{array}$ & $\begin{array}{r}576 \cdot 0(310 \cdot 1 \\
\text { to } 972 \cdot 6)\end{array}$ & $\begin{array}{r}-27 \cdot 6^{*}(-39 \cdot 4 \text { to } \\
-10 \cdot 4)\end{array}$ & $\begin{array}{r}-45.9^{*}(-55.0 \text { to } \\
-32.8)\end{array}$ \\
\hline Hookworm disease & $\begin{array}{r}471816 \cdot 2(437 \\
049 \cdot 6 \text { to } 511 \\
318 \cdot 8)\end{array}$ & $\begin{array}{r}-5 \cdot 1(-20 \cdot 2 \text { to } \\
9 \cdot 7)\end{array}$ & $\begin{array}{r}-30 \cdot 1 *(-41 \cdot 6 \text { to } \\
-18 \cdot 4)\end{array}$ & $\begin{array}{r}2181.7 \\
(1338.6 \text { to } \\
3354.5)\end{array}$ & $\begin{array}{r}-10 \cdot 9 *(-17 \cdot 3 \text { to } \\
-4 \cdot 4)\end{array}$ & $\begin{array}{r}-32.9 *(-38.0 \text { to } \\
-27.7)\end{array}$ \\
\hline Food-borne trematodiases & $\begin{array}{r}80194 \cdot 5(64 \\
648 \cdot 1 \text { to } 96 \\
212 \cdot 4)\end{array}$ & $\begin{array}{r}51.1 *(44.2 \text { to } \\
59.7)\end{array}$ & $-0 \cdot 1(-4 \cdot 8$ to $5 \cdot 6)$ & $\begin{array}{r}3634 \cdot 8 \\
(1160 \cdot 2 \text { to } \\
7692 \cdot 4)\end{array}$ & $\begin{array}{r}50 \cdot 1 *{ }^{*}(40.6 \text { to } \\
60 \cdot 0)\end{array}$ & $-1.8(-8.1$ to 4.9$)$ \\
\hline Other neglected tropical diseases & $\begin{array}{r}59705 \cdot 9(58 \\
703 \cdot 5 \text { to } 61 \\
020 \cdot 1)\end{array}$ & $\begin{array}{r}-5 \cdot 0(-11 \cdot 0 \text { to } \\
1 \cdot 6)\end{array}$ & $\begin{array}{r}-18 \cdot 3^{*}(-23 \cdot 3 \text { to } \\
-12 \cdot 8)\end{array}$ & $\begin{array}{r}2260 \cdot 7 \\
(1473 \cdot 6 \text { to } \\
3358 \cdot 6)\end{array}$ & $\begin{array}{r}-8.9 *(-13 \cdot 4 \text { to } \\
-0 \cdot 1)\end{array}$ & $\begin{array}{r}-20.6^{*}(-25.0 \text { to } \\
-12.8)\end{array}$ \\
\hline Maternal disorders & & .. & .. & $\begin{array}{r}1341 \cdot 8 \\
(924 \cdot 5 \text { to } \\
1824 \cdot 3)\end{array}$ & $\begin{array}{r}18 \cdot 4 *(8 \cdot 2 \text { to } \\
27 \cdot 4)\end{array}$ & $\begin{array}{r}-15 \cdot 0 *(-21 \cdot 9 \text { to } \\
-8 \cdot 4)\end{array}$ \\
\hline Maternal haemorrhage & $\begin{array}{r}2027 \cdot 4(1932 \cdot 4 \\
\text { to } 2119 \cdot 8)\end{array}$ & $\begin{array}{r}30.5^{*}(22 \cdot 2 \text { to } \\
39 \cdot 2)\end{array}$ & $-2 \cdot 1(-8 \cdot 1$ to $4 \cdot 4)$ & $\begin{array}{r}69.5(47.4 \text { to } \\
97.5)\end{array}$ & $\begin{array}{r}11 \cdot 2(-0.7 \text { to } \\
22 \cdot 5)\end{array}$ & 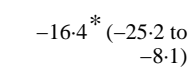 \\
\hline $\begin{array}{l}\text { Maternal sepsis and other maternal } \\
\text { infections }\end{array}$ & $\begin{array}{r}1781.4(1235.4 \\
\text { to } 2515.9)\end{array}$ & $\begin{array}{r}-13 \cdot 5^{*}(-23 \cdot 5 \\
\text { to }-5 \cdot 0)\end{array}$ & $\begin{array}{r}-41.6 *(-47.7 \text { to } \\
-35.6)\end{array}$ & $\begin{array}{r}21 \cdot 4(11 \cdot 6 \text { to } \\
36 \cdot 3)\end{array}$ & 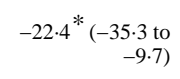 & $\begin{array}{r}-43 \cdot 2 *(-51 \cdot 8 \text { to } \\
-34 \cdot 5)\end{array}$ \\
\hline Maternal hypertensive disorders & $\begin{array}{r}1277 \cdot 6(756 \cdot 3 \\
\text { to } 1978 \cdot 8)\end{array}$ & $\begin{array}{r}3.5(-0.4 \text { to } \\
10.2)\end{array}$ & 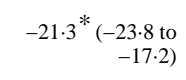 & $\begin{array}{r}64.7(31.5 \text { to } \\
112.8)\end{array}$ & $2 \cdot 9(-2 \cdot 8$ to $9 \cdot 7)$ & $\begin{array}{r}-21 \cdot 8^{*}(-25 \cdot 6 \text { to } \\
-17 \cdot 3)\end{array}$ \\
\hline Obstructed labour & $\begin{array}{r}2902 \cdot 1(2600 \cdot 1 \\
\text { to } 3222 \cdot 1)\end{array}$ & $\begin{array}{r}20.4 *(12.9 \text { to } \\
27.5)\end{array}$ & $\begin{array}{r}-14 \cdot 8^{*}(-19 \cdot 8 \text { to } \\
-10 \cdot 2)\end{array}$ & $\begin{array}{r}951.5(641.5 \\
\text { to } 1304 \cdot 0)\end{array}$ & $\begin{array}{r}20.9^{*}(12.3 \text { to } \\
30 \cdot 3)\end{array}$ & 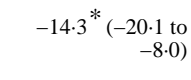 \\
\hline Complications of abortion & $\begin{array}{r}21.8(14.6 \text { to } \\
29 \cdot 7)\end{array}$ & $\begin{array}{r}11.5 *{ }^{*}(1.8 \text { to } \\
20.9)\end{array}$ & $\begin{array}{r}-16.9^{*}(-24 \cdot 0 \text { to } \\
-10 \cdot 1)\end{array}$ & $\begin{array}{r}2.5(1.4 \text { to } \\
4.0)\end{array}$ & $\begin{array}{r}12.0 *(2.0 \text { to } \\
22 \cdot 3)\end{array}$ & 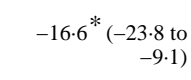 \\
\hline Other maternal disorders & .. & .. & .. & $\begin{array}{r}232 \cdot 2(155 \cdot 8 \\
\text { to } 343 \cdot 7)\end{array}$ & $\begin{array}{r}22 \cdot 1(-14 \cdot 3 \text { to } \\
62 \cdot 2)\end{array}$ & $\begin{array}{r}-10 \cdot 5(-37.3 \text { to } \\
18 \cdot 5)\end{array}$ \\
\hline Neonatal disorders & .. & .. & .. & $\begin{array}{r}12648 \cdot 8(92 \\
91 \cdot 4 \text { to } 16 \\
262 \cdot 6)\end{array}$ & $\begin{array}{r}130 \cdot 9^{*}(108 \cdot 5 \text { to } \\
158 \cdot 7)\end{array}$ & $80 \cdot 5^{*}\left(\begin{array}{c}63 \cdot 1 \text { to } \\
101 \cdot 7)\end{array}\right.$ \\
\hline Preterm birth complications & $\begin{array}{r}56272 \cdot 0(49 \\
504 \cdot 6 \text { to } 64 \\
204 \cdot 4)\end{array}$ & $\begin{array}{r}165.4^{*}(143.0 \text { to } \\
192.5)\end{array}$ & $\begin{array}{r}98.9^{*}(81.9 \text { to } \\
118.4)\end{array}$ & $\begin{array}{r}6689 \cdot 7 \\
(4914 \cdot 1 \text { to } \\
8643 \cdot 6)\end{array}$ & $\begin{array}{r}187 \cdot 6^{*}(166 \cdot 9 \text { to } \\
210 \cdot 3)\end{array}$ & $\begin{array}{r}119 \cdot 4^{*}(104 \cdot 2 \text { to } \\
136 \cdot 9)\end{array}$ \\
\hline $\begin{array}{l}\text { Neonatal encephalopathy due to } \\
\text { birth asphyxia and trauma }\end{array}$ & $\begin{array}{r}22858.6(11 \\
831 \cdot 4 \text { to } 38 \\
618.7)\end{array}$ & $\begin{array}{r}3.4(-18.7 \text { to } \\
53.4)\end{array}$ & $\begin{array}{r}-20.5(-37.6 \text { to } \\
19 \cdot 2)\end{array}$ & $\begin{array}{r}2388 \cdot 1 \\
(1783 \cdot 6 \text { to } \\
3153 \cdot 0)\end{array}$ & $\begin{array}{r}57 \cdot 1 *(32 \cdot 4 \text { to } \\
84 \cdot 0)\end{array}$ & $25 \cdot 6 *(5.4$ to $48 \cdot 1)$ \\
\hline $\begin{array}{l}\text { Neonatal sepsis and other neonatal } \\
\text { infections }\end{array}$ & $\begin{array}{r}50 \cdot 3(16 \cdot 5 \text { to } \\
106 \cdot 6)\end{array}$ & 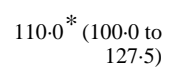 & $\begin{aligned} & 107.7^{*}(97.8 \text { to } \\
&125.0)\end{aligned}$ & $\begin{array}{r}6 \cdot 6(2 \cdot 0 \text { to } \\
15 \cdot 0)\end{array}$ & 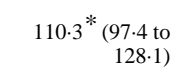 & $108 \cdot 1 * \begin{array}{r}(95.3 \text { to } \\
125.7)\end{array}$ \\
\hline $\begin{array}{l}\text { Haemolytic disease and other } \\
\text { neonatal jaundice }\end{array}$ & $\begin{array}{r}4999 \cdot 3(3893 \cdot 0 \\
\text { to } 6792 \cdot 3)\end{array}$ & $\begin{array}{r}132.9^{*}(66.8 \text { to } \\
276.7)\end{array}$ & $\begin{array}{r}84.2^{*}(33.6 \text { to } \\
197.8)\end{array}$ & $\begin{array}{r}1606 \cdot 8 \\
(1122 \cdot 3 \text { to } \\
2234 \cdot 6)\end{array}$ & $\begin{array}{r}112 \cdot 4^{*}(61 \cdot 1 \text { to } \\
220 \cdot 9)\end{array}$ & 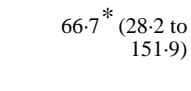 \\
\hline Other neonatal disorders & .. & .. & .. & $\begin{array}{r}1957 \cdot 6 \\
(1334.4 \text { to } \\
2698.5)\end{array}$ & $\begin{array}{r}128 \cdot 8 *(82 \cdot 2 \text { to } \\
189 \cdot 1)\end{array}$ & $\begin{array}{r}80 \cdot 3^{*}(43 \cdot 8 \text { to } \\
126 \cdot 6)\end{array}$ \\
\hline Nutritional deficiencies & .. & .. & .. & $\begin{array}{r}41765 \cdot 3(27 \\
678 \cdot 6 \text { to } 60 \\
351 \cdot 7)\end{array}$ & $\begin{array}{r}-9 \cdot 4 *(-12 \cdot 0 \text { to } \\
-7 \cdot 0)\end{array}$ & $\begin{array}{r}-26 \cdot 1^{*}(-27 \cdot 8 \text { to } \\
-24 \cdot 5)\end{array}$ \\
\hline
\end{tabular}

Lancet. Author manuscript; available in PMC 2015 September 07. 


\begin{tabular}{|c|c|c|c|c|c|c|}
\hline & $\begin{array}{l}\text { Prevalent cases } \\
\text { in 2013 }(x \\
1000)\end{array}$ & $\begin{array}{l}\text { Percentage } \\
\text { change in } \\
\text { prevalence } \\
\text { from } 1990 \text { to } \\
2013\end{array}$ & $\begin{array}{l}\text { Percentage } \\
\text { change in age- } \\
\text { standardised } \\
\text { prevalence from } \\
1990 \text { to } 2013\end{array}$ & $\begin{array}{l}\text { YLDs in } \\
2013(x \\
1000)\end{array}$ & $\begin{array}{l}\text { Percentage } \\
\text { change in YLDs } \\
\text { from } 1990 \text { to } \\
2013\end{array}$ & $\begin{array}{l}\text { Percentage change } \\
\text { in age- } \\
\text { standardised } \\
\text { YLDs from } 1990 \\
\text { to } 2013\end{array}$ \\
\hline Protein-energy malnutrition & $\begin{array}{r}20756 \cdot 9(16 \\
985 \cdot 3 \text { to } 25 \\
759 \cdot 4)\end{array}$ & $\begin{array}{r}-13 \cdot 2(-34 \cdot 7 \text { to } \\
16 \cdot 1)\end{array}$ & $\begin{array}{r}-16 \cdot 7(-37.0 \text { to } \\
11 \cdot 6)\end{array}$ & $\begin{array}{r}2574 \cdot 3 \\
(1588 \cdot 3 \text { to } \\
3920 \cdot 3)\end{array}$ & $\begin{array}{r}-13.0(-34.9 \text { to } \\
16.9)\end{array}$ & $\begin{array}{r}-16 \cdot 3(-37 \cdot 0 \text { to } \\
12 \cdot 3)\end{array}$ \\
\hline Iodine deficiency & $\begin{array}{r}115602 \cdot 4(110 \\
007 \cdot 2 \text { to } 120 \\
997 \cdot 1)\end{array}$ & $\begin{array}{r}-11 \cdot 4 *(-16.8 \\
\text { to }-5 \cdot 4)\end{array}$ & $\begin{array}{r}-37 \cdot 0 *(-40 \cdot 9 \text { to } \\
-32 \cdot 5)\end{array}$ & $\begin{array}{r}2075 \cdot 0 \\
(1294.6 \text { to } \\
3291.9)\end{array}$ & $\begin{array}{r}-11 \cdot 4^{*}(-17 \cdot 1 \text { to } \\
-5 \cdot 4)\end{array}$ & $\begin{array}{r}-36 \cdot 7^{*}(-40 \cdot 8 \text { to } \\
-32 \cdot 3)\end{array}$ \\
\hline Vitamin A deficiency & $\begin{array}{r}3372 \cdot 4(2732 \cdot 4 \\
\text { to } 3926 \cdot 3)\end{array}$ & $\begin{array}{r}-19 \cdot 4^{*}(-24 \cdot 4 \\
\text { to }-14 \cdot 6)\end{array}$ & $\begin{array}{r}-34.9^{*}(-39 \cdot 3 \text { to } \\
-31 \cdot 1)\end{array}$ & $\begin{array}{r}153.7(99.0 \\
\text { to } 224.9)\end{array}$ & $\begin{array}{r}-22.9^{*}(-28.7 \text { to } \\
-16.9)\end{array}$ & $\begin{array}{r}-39 \cdot 1^{*}(-44 \cdot 1 \text { to } \\
-33 \cdot 5)\end{array}$ \\
\hline Iron-deficiency anaemia & $\begin{array}{r}1208216 \cdot 4(1 \\
205927 \cdot 2 \text { to } 1 \\
210477 \cdot 8)\end{array}$ & $\begin{array}{r}-0 \cdot 2(-0 \cdot 6 \text { to } \\
0 \cdot 1)\end{array}$ & $\begin{array}{r}-21.9 *(-22.2 \text { to } \\
-21.7)\end{array}$ & $\begin{array}{r}36663.5(24 \\
371.0 \text { to } 53 \\
084.7)\end{array}$ & $\begin{array}{r}-8.6^{*}(-9.8 \text { to } \\
-7 \cdot 1)\end{array}$ & $\begin{array}{r}-25.8^{*}(-26.7 \text { to } \\
-24.9)\end{array}$ \\
\hline Other nutritional deficiencies & .. & & & $\begin{array}{r}298.8(157.9 \\
\text { to } 535.4)\end{array}$ & $\begin{array}{r}-43 \cdot 8^{*}(-63 \cdot 9 \text { to } \\
-10.0)\end{array}$ & 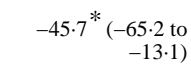 \\
\hline $\begin{array}{l}\text { Other communicable, maternal, } \\
\text { noonatal, and nutritional diseases }\end{array}$ & .. & .. & & $\begin{array}{r}3565 \cdot 4 \\
(2282 \cdot 3 \text { to } \\
5460 \cdot 0)\end{array}$ & $9 \cdot 7^{*}(5 \cdot 6$ to $15 \cdot 8)$ & $\begin{array}{r}-13 \cdot 4^{*}(-15 \cdot 8 \text { to } \\
-9 \cdot 9)\end{array}$ \\
\hline $\begin{array}{l}\text { Sexually transmitted diseases } \\
\text { excluding HIV }\end{array}$ &.. &.. &.. & $\begin{array}{r}1383 \cdot 8 \\
(830 \cdot 1 \text { to } \\
2404 \cdot 6)\end{array}$ & $\begin{array}{r}38.0 *(33.1 \text { to } \\
42.5)\end{array}$ & $-2 \cdot 6(-5 \cdot 6$ to 0.5$)$ \\
\hline Syphilis & $\begin{array}{r}315 \cdot 2(306 \cdot 5 \text { to } \\
324 \cdot 5)\end{array}$ & $1.5(-2.5$ to 6.4$)$ & 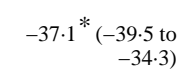 & $\begin{array}{r}58.4(39 \cdot 6 \text { to } \\
81 \cdot 8)\end{array}$ & $1.5(-5.3$ to 9.9$)$ & $\begin{array}{r}-36.9^{*}(-40.8 \text { to } \\
-32.0)\end{array}$ \\
\hline Chlamydial infection & $\begin{array}{r}147846.5(144 \\
169 \cdot 7 \text { to } 151 \\
027.5)\end{array}$ & 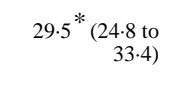 & $\begin{array}{r}-5.5 *(-8.8 \text { to } \\
-2.8)\end{array}$ & $\begin{array}{r}646.5(411.0 \\
\text { to } 1019 \cdot 4)\end{array}$ & $\begin{array}{r}37 \cdot 1 *(31 \cdot 0 \text { to } \\
43 \cdot 3)\end{array}$ & $0.4(-4 \cdot 2$ to 4.8$)$ \\
\hline Gonococcal infection & $\begin{array}{r}32726 \cdot 1(31 \\
162 \cdot 7 \text { to } 34 \\
316 \cdot 7)\end{array}$ & $\begin{array}{r}38.8^{*}(29.6 \text { to } \\
47.6)\end{array}$ & $4.8(-2.0$ to 11.4$)$ & $\begin{array}{r}225 \cdot 4(144 \cdot 8 \\
\text { to } 344 \cdot 1)\end{array}$ & $\begin{array}{r}43 \cdot 4^{*}(29 \cdot 1 \text { to } \\
61 \cdot 0)\end{array}$ & $7.9(-2.6$ to 20.9$)$ \\
\hline Trichomoniasis & $\begin{array}{r}67075.9(62 \\
762 \cdot 6 \text { to } 72 \\
764 \cdot 6)\end{array}$ & $\begin{array}{r}45 \cdot 6 *(33.3 \text { to } \\
62 \cdot 3)\end{array}$ & $4 \cdot 0(-4 \cdot 4$ to $15 \cdot 6)$ & $\begin{array}{r}113 \cdot 9(45 \cdot 1 \\
\text { to } 242 \cdot 9)\end{array}$ & $\begin{array}{r}45.5^{*}(32.3 \text { to } \\
64 \cdot 1)\end{array}$ & $4 \cdot 2(-4.9$ to 16.9$)$ \\
\hline Genital herpes & $\begin{array}{r}1176494 \cdot 5(1 \\
160277 \cdot 4 \text { to } 1 \\
194517 \cdot 2)\end{array}$ & $\begin{array}{r}47.6 *(44.7 \text { to } \\
50 \cdot 6)\end{array}$ & $\begin{array}{r}-5 \cdot 3 *(-7 \cdot 1 \text { to } \\
-3 \cdot 5)\end{array}$ & $\begin{array}{r}311 \cdot 6(98 \cdot 3 \\
\text { to } 748 \cdot 5)\end{array}$ & $\begin{array}{r}45 \cdot 7^{*}(40 \cdot 8 \text { to } \\
49 \cdot 6)\end{array}$ & $\begin{array}{r}-4 \cdot 8^{*}(-6 \cdot 8 \text { to } \\
-2 \cdot 6)\end{array}$ \\
\hline $\begin{array}{l}\text { Other sexually transmitted } \\
\text { diseases }\end{array}$ & $\begin{array}{r}1248 \cdot 2(971 \cdot 7 \\
\text { to } 1612 \cdot 4)\end{array}$ & $\begin{array}{r}1.9(-4 \cdot 1 \text { to } \\
10 \cdot 2)\end{array}$ & 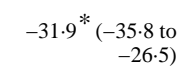 & $\begin{array}{r}27.9(19 \cdot 0 \text { to } \\
40 \cdot 2)\end{array}$ & $20.0 *\left(\begin{array}{r}(9.2 \text { to } \\
32 \cdot 4)\end{array}\right.$ & 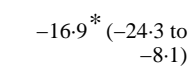 \\
\hline Hepatitis & .. & .. & & $\begin{array}{r}444 \cdot 1(290 \cdot 5 \\
\text { to } 641 \cdot 1)\end{array}$ & $\begin{array}{r}14.9 *(12.1 \text { to } \\
17.5)\end{array}$ & $\begin{array}{r}-17.3^{*}(-19.4 \text { to } \\
-15.3)\end{array}$ \\
\hline Hepatitis A & $\begin{array}{r}7823.9(7532.8 \\
\text { to } 8115 \cdot 3)\end{array}$ & $\begin{array}{r}11.9^{*}(11.3 \text { to } \\
12.5)\end{array}$ & $\begin{array}{r}-5.8 *(-6 \cdot 1 \text { to } \\
-5 \cdot 3)\end{array}$ & $\begin{array}{r}198.0(128.3 \\
\text { to } 287.8)\end{array}$ & $\begin{array}{r}25.9 *(22.9 \text { to } \\
29.1)\end{array}$ & $-0.9(-3.5$ to $2 \cdot 0)$ \\
\hline Hepatitis B & $\begin{array}{r}331037.0(325 \\
359 \cdot 6 \text { to } 336 \\
638 \cdot 4)\end{array}$ & $\begin{array}{r}-5.5^{*}(-7.9 \text { to } \\
-3 \cdot 4)\end{array}$ & $\begin{array}{r}-32.1 *(-33 \cdot 7 \text { to } \\
-30 \cdot 6)\end{array}$ & $\begin{array}{r}172 \cdot 6(112 \cdot 0 \\
\text { to } 247 \cdot 4)\end{array}$ & $2 \cdot 2(-2.3$ to $6 \cdot 7)$ & $\begin{array}{r}-31.8 *(-35.0 \text { to } \\
-28.6)\end{array}$ \\
\hline Hepatitis C & $\begin{array}{r}147826 \cdot 3(145 \\
520 \cdot 7 \text { to } 150 \\
080 \cdot 2)\end{array}$ & $\begin{array}{r}16.0^{*}(13.6 \text { to } \\
18.5)\end{array}$ & $\begin{array}{r}-23 \cdot 3^{*}(-24 \cdot 8 \text { to } \\
-21 \cdot 7)\end{array}$ & $\begin{array}{r}16.9(11.0 \text { to } \\
24.3)\end{array}$ & $\begin{array}{r}15.5^{*}(10 \cdot 0 \text { to } \\
21.4)\end{array}$ & $\begin{array}{r}-21 \cdot 2 *(-25 \cdot 1 \text { to } \\
-17 \cdot 1)\end{array}$ \\
\hline Hepatitis E & $\begin{array}{r}2188.2(2083.3 \\
\text { to } 2311 \cdot 9)\end{array}$ & $\begin{array}{r}18.4^{*}(11.3 \text { to } \\
26.9)\end{array}$ & $\begin{array}{r}-10.9^{*}(-16 \cdot 2 \text { to } \\
-4 \cdot 6)\end{array}$ & $\begin{array}{r}56 \cdot 6(36 \cdot 3 \text { to } \\
82 \cdot 1)\end{array}$ & 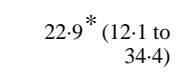 & $\begin{array}{r}-8 \cdot 6^{*}(-16 \cdot 4 \text { to } \\
-0.0)\end{array}$ \\
\hline Leprosy & $\begin{array}{r}658.8(613.6 \text { to } \\
707.5)\end{array}$ & $\begin{array}{r}61 \cdot 3^{*}(54 \cdot 0 \text { to } \\
69 \cdot 6)\end{array}$ & $-1.5(-6.0$ to 3.5$)$ & $\begin{array}{r}39.7(26.6 \text { to } \\
56 \cdot 0)\end{array}$ & $\begin{array}{r}73 \cdot 4^{*}(61 \cdot 0 \text { to } \\
86 \cdot 6)\end{array}$ & $5 \cdot 7(-1.5$ to $13 \cdot 4)$ \\
\hline Other infectious diseases & $\begin{array}{r}49759 \cdot 6(48 \\
625 \cdot 5 \text { to } 51 \\
029 \cdot 0)\end{array}$ & $\begin{array}{r}-4.9^{*}(-7.8 \text { to } \\
-2.0)\end{array}$ & $\begin{array}{r}-20 \cdot 6^{*}(-22 \cdot 9 \text { to } \\
-18 \cdot 2)\end{array}$ & $\begin{array}{r}1697.9 \\
(1130.0 \text { to } \\
2469.8)\end{array}$ & $\begin{array}{r}-7.5^{*}(-11 \cdot 7 \text { to } \\
-1 \cdot 1)\end{array}$ & $\begin{array}{r}-19.8^{*}(-23.4 \text { to } \\
-14 \cdot 6)\end{array}$ \\
\hline Non-communicable diseases & .. &.. & .. & $\begin{array}{r}626477 \cdot 7 \\
(465287 \cdot 7 \text { to } \\
806573 \cdot 8)\end{array}$ & $\begin{array}{r}54 \cdot 2 *{ }^{*}(53 \cdot 0 \text { to } \\
55 \cdot 8)\end{array}$ & $1 \cdot 4 *(0 \cdot 7$ to $2 \cdot 2)$ \\
\hline
\end{tabular}




\begin{tabular}{|c|c|c|c|c|c|c|}
\hline & $\begin{array}{l}\text { Prevalent cases } \\
\text { in 2013 }(x \\
1000)\end{array}$ & $\begin{array}{l}\text { Percentage } \\
\text { change in } \\
\text { prevalence } \\
\text { from } 1990 \text { to } \\
2013\end{array}$ & $\begin{array}{l}\text { Percentage } \\
\text { change in age- } \\
\text { standardised } \\
\text { prevalence from } \\
1990 \text { to } 2013\end{array}$ & $\begin{array}{l}\text { YLDs in } \\
2013(x \\
1000)\end{array}$ & $\begin{array}{l}\text { Percentage } \\
\text { change in YLDs } \\
\text { from } 1990 \text { to } \\
2013\end{array}$ & $\begin{array}{l}\text { Percentage change } \\
\text { in age- } \\
\text { standardised } \\
\text { YLDs from } 1990 \\
\text { to } 2013\end{array}$ \\
\hline Neoplasms & .. & .. & .. & $\begin{array}{r}6763 \cdot 9 \\
(4989 \cdot 2 \text { to } \\
8715 \cdot 9)\end{array}$ & $\begin{array}{r}82 \cdot 5 *(75 \cdot 4 \text { to } \\
90 \cdot 8)\end{array}$ & $8 \cdot 5^{*}(4 \cdot 4$ to $13 \cdot 5)$ \\
\hline Oesophageal cancer & $\begin{array}{r}840 \cdot 5(706 \cdot 3 \text { to } \\
1015 \cdot 2)\end{array}$ & $\begin{array}{r}56 \cdot 2^{*}(30 \cdot 8 \text { to } \\
90 \cdot 0)\end{array}$ & $-9.5(-24.2$ to 9.9$)$ & $\begin{array}{r}125 \cdot 7(88.9 \\
\text { to } 167 \cdot 2)\end{array}$ & $49 \cdot 6^{*}\left(\begin{array}{r}30 \cdot 2 \text { to } \\
72 \cdot 8)\end{array}\right.$ & $\begin{array}{r}-13 \cdot 6^{*}(-24.4 \text { to } \\
-0 \cdot 3)\end{array}$ \\
\hline Stomach cancer & $\begin{array}{r}2532 \cdot 1(2347 \cdot 7 \\
\text { to } 2736 \cdot 5)\end{array}$ & $\begin{array}{r}36.1 *(25.8 \text { to } \\
47.4)\end{array}$ & $\begin{array}{r}-21 \cdot 0 *(-26 \cdot 9 \text { to } \\
-14.7)\end{array}$ & $\begin{array}{r}290 \cdot 4(209 \cdot 4 \\
\text { to } 368 \cdot 1)\end{array}$ & $\begin{array}{r}28 \cdot 2 *(19 \cdot 1 \text { to } \\
38 \cdot 3)\end{array}$ & $\begin{array}{r}-25.7^{*}(-30 \cdot 9 \text { to } \\
-20 \cdot 0)\end{array}$ \\
\hline Liver cancer &.. &.. & .. & $\begin{array}{r}190 \cdot 6(133 \cdot 3 \\
\text { to } 253 \cdot 2)\end{array}$ & $\begin{array}{r}76.9 *(55.6 \text { to } \\
102 \cdot 0)\end{array}$ & $4.5(-7.6$ to 19.0$)$ \\
\hline Liver cancer due to hepatitis B & $\begin{array}{r}450 \cdot 9(373.3 \text { to } \\
538.8)\end{array}$ & $\begin{array}{r}91.2^{*}(48.7 \text { to } \\
150 \cdot 7)\end{array}$ & $\begin{array}{r}14 \cdot 2(-10 \cdot 8 \text { to } \\
48 \cdot 5)\end{array}$ & $\begin{array}{r}67.0(46.3 \text { to } \\
89.9)\end{array}$ & $\begin{array}{r}70 \cdot 7^{*}(44 \cdot 5 \text { to } \\
102 \cdot 0)\end{array}$ & $2 \cdot 0(-13 \cdot 6$ to $20 \cdot 5)$ \\
\hline Liver cancer due to hepatitis $\mathrm{C}$ & $\begin{array}{r}512 \cdot 6(438.6 \text { to } \\
599 \cdot 8)\end{array}$ & $\begin{array}{r}367.6^{*}(284.7 \text { to } \\
478.2)\end{array}$ & $\begin{array}{r}168.7^{*}(120 \cdot 7 \text { to } \\
230 \cdot 0)\end{array}$ & $\begin{array}{r}74.6(52.6 \text { to } \\
99.9)\end{array}$ & $\begin{array}{r}310.5 *(256.5 \text { to } \\
378.5)\end{array}$ & $\begin{array}{r}135.7^{*}(106 \cdot 8 \text { to } \\
174 \cdot 3)\end{array}$ \\
\hline Liver cancer due to alcohol use & $\begin{array}{r}197 \cdot 2(168 \cdot 7 \text { to } \\
226 \cdot 8)\end{array}$ & $\begin{array}{r}10 \cdot 2(-8 \cdot 8 \text { to } \\
34 \cdot 4)\end{array}$ & $\begin{array}{r}-35 \cdot 8 *(-46 \cdot 5 \text { to } \\
-22 \cdot 2)\end{array}$ & $\begin{array}{r}29.9(20 \cdot 7 \text { to } \\
40 \cdot 1)\end{array}$ & $\begin{array}{r}2 \cdot 0(-10 \cdot 3 \text { to } \\
16 \cdot 3)\end{array}$ & $\begin{array}{r}-40 \cdot 4^{*}(-47 \cdot 6 \text { to } \\
-32 \cdot 1)\end{array}$ \\
\hline Liver cancer due to other causes & $\begin{array}{r}121 \cdot 5(102 \cdot 1 \text { to } \\
143 \cdot 0)\end{array}$ & $\begin{array}{r}-1 \cdot 0(-23 \cdot 1 \text { to } \\
22 \cdot 3)\end{array}$ & $\begin{array}{r}-40 \cdot 7^{*}(-54 \cdot 1 \text { to } \\
-27 \cdot 0)\end{array}$ & $\begin{array}{r}19 \cdot 1(13 \cdot 1 \text { to } \\
25 \cdot 6)\end{array}$ & $\begin{array}{r}-9 \cdot 8(-24 \cdot 0 \text { to } \\
4 \cdot 4)\end{array}$ & $\begin{array}{r}-45.8^{*}(-54.2 \text { to } \\
-37.5)\end{array}$ \\
\hline Larynx cancer & $\begin{array}{r}899.8(749.7 \text { to } \\
1069 \cdot 8)\end{array}$ & $\begin{array}{r}41 \cdot 6^{*}(30 \cdot 2 \text { to } \\
58 \cdot 1)\end{array}$ & $\begin{array}{r}-16 \cdot 6^{*}(-23 \cdot 0 \text { to } \\
-7 \cdot 1)\end{array}$ & $\begin{array}{r}86 \cdot 0(60 \cdot 0 \text { to } \\
115 \cdot 2)\end{array}$ & $\begin{array}{r}32.8 *(22.0 \text { to } \\
49 \cdot 6)\end{array}$ & $\begin{array}{r}-22.0 *(-28.3 \text { to } \\
-12.4)\end{array}$ \\
\hline Tracheal, bronchus, and lung cancer & $\begin{array}{r}3227 \cdot 4(3039 \cdot 7 \\
\text { to } 3426 \cdot 8)\end{array}$ & $\begin{array}{r}72 \cdot 2^{*}(61 \cdot 9 \text { to } \\
82 \cdot 1)\end{array}$ & $0 \cdot 8(-5 \cdot 4$ to $6 \cdot 5)$ & $\begin{array}{r}467 \cdot 4(338.5 \\
\text { to } 593 \cdot 2)\end{array}$ & $\begin{array}{r}64.6 *(54.9 \text { to } \\
72.9)\end{array}$ & $-3 \cdot 7(-9 \cdot 3$ to $1 \cdot 2)$ \\
\hline Breast cancer & $\begin{array}{r}18419 \cdot 0(17 \\
740 \cdot 6 \text { to } 19 \\
141 \cdot 4)\end{array}$ & $\begin{array}{r}126.9^{*}(115.5 \text { to } \\
139.4)\end{array}$ & $\begin{array}{r}29 \cdot 7^{*}(23.5 \text { to } \\
36 \cdot 5)\end{array}$ & $\begin{array}{r}1068 \cdot 2 \\
(760 \cdot 8 \text { to } \\
1428 \cdot 1)\end{array}$ & $\begin{array}{r}100 \cdot 7^{*}(86 \cdot 6 \text { to } \\
114 \cdot 3)\end{array}$ & $15 \cdot 7^{*}(7 \cdot 8$ to $23 \cdot 2)$ \\
\hline Cervical cancer & $\begin{array}{r}3180 \cdot 8(2623 \cdot 1 \\
\text { to } 3599 \cdot 0)\end{array}$ & $\begin{array}{r}7 \cdot 2(-5.4 \text { to } \\
21.9)\end{array}$ & $\begin{array}{r}-35 \cdot 2^{*}(-42 \cdot 6 \text { to } \\
-26 \cdot 8)\end{array}$ & $\begin{array}{r}243 \cdot 8(169 \cdot 8 \\
\text { to } 333 \cdot 0)\end{array}$ & $8 \cdot 3(-3 \cdot 5$ to $22 \cdot 3)$ & $\begin{array}{r}-34.6^{*}(-41.7 \text { to } \\
-26 \cdot 6)\end{array}$ \\
\hline Uterine cancer & $\begin{array}{r}2960 \cdot 8(2307.9 \\
\text { to } 3516 \cdot 4)\end{array}$ & $\begin{array}{r}66.5^{*}(39.0 \text { to } \\
97.8)\end{array}$ & $\begin{array}{r}-3 \cdot 8(-19 \cdot 0 \text { to } \\
13 \cdot 3)\end{array}$ & $\begin{array}{r}193.7(123.7 \\
\text { to } 273.6)\end{array}$ & $\begin{array}{r}62.9^{*}(36.4 \text { to } \\
92 \cdot 1)\end{array}$ & $-6 \cdot 0(-20 \cdot 6$ to $9 \cdot 8)$ \\
\hline Prostate cancer & $\begin{array}{r}11135 \cdot 9(10 \\
002 \cdot 5 \text { to } 13 \\
123 \cdot 4)\end{array}$ & $\begin{array}{r}178 \cdot 8^{*}(158 \cdot 1 \text { to } \\
212 \cdot 7)\end{array}$ & $\begin{array}{r}56 \cdot 0^{*}(44.6 \text { to } \\
75.0)\end{array}$ & $\begin{array}{r}893.7(656 \cdot 7 \\
\text { to } 1192 \cdot 5)\end{array}$ & $\begin{array}{r}164 \cdot 2 *(142.0 \text { to } \\
208 \cdot 3)\end{array}$ & $48 \cdot 2^{*}(35.8$ to 72.9$)$ \\
\hline Colon and rectum cancer & $\begin{array}{r}8591.6(8252.7 \\
\text { to } 8917.0)\end{array}$ & $\begin{array}{r}107.2^{*}(97.8 \text { to } \\
115.6)\end{array}$ & $\begin{array}{r}18 \cdot 4^{*}(13 \cdot 1 \text { to } \\
23 \cdot 2)\end{array}$ & $\begin{array}{r}701 \cdot 9(512 \cdot 7 \\
\text { to } 899 \cdot 1)\end{array}$ & $\begin{array}{r}95 \cdot 5^{*}(86 \cdot 5 \text { to } \\
104 \cdot 1)\end{array}$ & $11.5^{*}(6.3$ to 16.5$)$ \\
\hline Lip and oral cavity cancer & $\begin{array}{r}2416 \cdot 5(2044 \cdot 4 \\
\text { to } 2782 \cdot 1)\end{array}$ & $\begin{array}{r}76 \cdot 8^{*}(56 \cdot 3 \text { to } \\
103 \cdot 4)\end{array}$ & $2 \cdot 9(-9 \cdot 0$ to $18 \cdot 2)$ & $\begin{array}{r}206 \cdot 9(145 \cdot 2 \\
\text { to } 278 \cdot 3)\end{array}$ & $\begin{array}{r}72.3^{*}(52.9 \text { to } \\
98.2)\end{array}$ & $0.0(-11 \cdot 1$ to $14 \cdot 6)$ \\
\hline Nasopharynx cancer & $\begin{array}{r}501 \cdot 1(422 \cdot 3 \text { to } \\
596 \cdot 2)\end{array}$ & $\begin{array}{r}29.7 *(5.8 \text { to } \\
58.5)\end{array}$ & $\begin{array}{r}-21 \cdot 1^{*}(-35 \cdot 7 \text { to } \\
-4 \cdot 0)\end{array}$ & $\begin{array}{r}47 \cdot 9(33 \cdot 1 \text { to } \\
64 \cdot 3)\end{array}$ & $\begin{array}{r}25.9 *(5.6 \text { to } \\
49.6)\end{array}$ & $\begin{array}{r}-23.5 *(-36.0 \text { to } \\
-9.4)\end{array}$ \\
\hline Other pharynx cancer & $\begin{array}{r}752.5(644.9 \text { to } \\
857 \cdot 1)\end{array}$ & $\begin{array}{r}81.2^{*}(53.9 \text { to } \\
109.4)\end{array}$ & $4 \cdot 7(-10 \cdot 9$ to $20 \cdot 6)$ & $\begin{array}{r}66 \cdot 0(45 \cdot 5 \text { to } \\
88 \cdot 5)\end{array}$ & $\begin{array}{r}75.5 *(51.3 \text { to } \\
101.5)\end{array}$ & $1 \cdot 4(-12 \cdot 6$ to $16 \cdot 3)$ \\
\hline Gallbladder and biliary tract cancer & $\begin{array}{r}158.3(135.9 \text { to } \\
177.6)\end{array}$ & $\begin{array}{r}46.7^{*}(29 \cdot 3 \text { to } \\
62 \cdot 7)\end{array}$ & $\begin{array}{r}-17 \cdot 1 *(-27 \cdot 2 \text { to } \\
-8 \cdot 0)\end{array}$ & $\begin{array}{r}39 \cdot 1(27 \cdot 0 \text { to } \\
52 \cdot 1)\end{array}$ & $\begin{array}{r}39.8^{*}(22.7 \text { to } \\
54.9)\end{array}$ & $\begin{array}{r}-20 \cdot 5^{*}(-30 \cdot 6 \text { to } \\
-11.5)\end{array}$ \\
\hline Pancreatic cancer & $\begin{array}{r}384.3(355.9 \text { to } \\
412 \cdot 9)\end{array}$ & $\begin{array}{r}100 \cdot 5^{*}(84.2 \text { to } \\
117 \cdot 8)\end{array}$ & $14 \cdot 3^{*}$ (5.2 to $\left.24 \cdot 5\right)$ & $\begin{array}{r}73 \cdot 6(52 \cdot 1 \text { to } \\
96 \cdot 2)\end{array}$ & $\begin{array}{r}92.1 *(80.6 \text { to } \\
103.7)\end{array}$ & $9.9^{*}(3.1$ to $16 \cdot 8)$ \\
\hline Malignant skin melanoma & $\begin{array}{r}2341 \cdot 5(1808 \cdot 4 \\
\text { to } 3151 \cdot 3)\end{array}$ & $\begin{array}{r}87.9^{*}(65.9 \text { to } \\
107 \cdot 1)\end{array}$ & $13.9 *(0.5$ to 24.9$)$ & $\begin{array}{r}137.9(91.7 \\
\text { to } 205.0)\end{array}$ & $\begin{array}{r}80.8^{*}(59.5 \text { to } \\
99.7)\end{array}$ & $9 \cdot 1(-3 \cdot 8$ to $20 \cdot 2)$ \\
\hline Non-melanoma skin cancer & $\begin{array}{r}5529 \cdot 6(4962 \cdot 1 \\
\text { to } 6204 \cdot 9)\end{array}$ & $\begin{array}{r}108.8^{*}(78.8 \text { to } \\
141.7)\end{array}$ & $18 \cdot 2^{*}(0 \cdot 3$ to $36 \cdot 9)$ & $\begin{array}{r}126 \cdot 2(82 \cdot 5 \\
\text { to } 188 \cdot 1)\end{array}$ & $\begin{array}{r}152 \cdot 8 *(116 \cdot 5 \text { to } \\
200 \cdot 2)\end{array}$ & $42 \cdot 5^{*}(21 \cdot 3$ to $70 \cdot 3)$ \\
\hline Ovarian cancer & $\begin{array}{r}1053.8(975.7 \\
\text { to } 1145 \cdot 0)\end{array}$ & $\begin{array}{r}68 \cdot 3^{*}(56 \cdot 1 \text { to } \\
82 \cdot 3)\end{array}$ & $-0.9(-8.1$ to $7 \cdot 0)$ & $\begin{array}{r}134.9(97.0 \\
\text { to } 174.6)\end{array}$ & $\begin{array}{r}65 \cdot 1 *(52 \cdot 0 \text { to } \\
79 \cdot 7)\end{array}$ & $-2 \cdot 9(-10 \cdot 7$ to $4 \cdot 8)$ \\
\hline Testicular cancer & $\begin{array}{r}556.0(423.6 \text { to } \\
681 \cdot 0)\end{array}$ & $\begin{array}{r}60 \cdot 8^{*}(32.1 \text { to } \\
84.9)\end{array}$ & $9 \cdot 4(-9 \cdot 7$ to $25 \cdot 6)$ & $\begin{array}{r}34 \cdot 3(22 \cdot 2 \text { to } \\
49 \cdot 0)\end{array}$ & $\begin{array}{r}57.6 *(30 \cdot 8 \text { to } \\
80 \cdot 5)\end{array}$ & $6 \cdot 6(-10 \cdot 8$ to $21 \cdot 9)$ \\
\hline
\end{tabular}

Lancet. Author manuscript; available in PMC 2015 September 07. 


\begin{tabular}{|c|c|c|c|c|c|c|}
\hline & $\begin{array}{l}\text { Prevalent cases } \\
\text { in 2013 }(x \\
1000)\end{array}$ & $\begin{array}{l}\text { Percentage } \\
\text { change in } \\
\text { prevalence } \\
\text { from } 1990 \text { to } \\
2013 \\
\end{array}$ & $\begin{array}{l}\text { Percentage } \\
\text { change in age- } \\
\text { standardised } \\
\text { prevalence from } \\
1990 \text { to } 2013\end{array}$ & $\begin{array}{l}\text { YLDs in } \\
2013(x \\
1000)\end{array}$ & $\begin{array}{l}\text { Percentage } \\
\text { change in YLDs } \\
\text { from } 1990 \text { to } \\
2013\end{array}$ & $\begin{array}{l}\text { Percentage change } \\
\text { in age- } \\
\text { standardised } \\
\text { YLDs from } 1990 \\
\text { to } 2013\end{array}$ \\
\hline Kidney cancer & $\begin{array}{r}1960.6(1829.8 \\
\text { to } 2099 \cdot 4)\end{array}$ & $\begin{array}{r}117.3^{*}(104.5 \text { to } \\
131.6)\end{array}$ & $\begin{array}{r}31.8^{*}(23.9 \text { to } \\
40.4)\end{array}$ & $\begin{array}{r}139 \cdot 2(99 \cdot 2 \\
\text { to } 185 \cdot 8)\end{array}$ & $\begin{array}{r}109.7^{*}(97.0 \text { to } \\
123.5)\end{array}$ & $26 \cdot{ }^{*}(18 \cdot 2$ to $34 \cdot 3)$ \\
\hline Bladder cancer & $\begin{array}{r}2359 \cdot 6(2077 \cdot 2 \\
\text { to } 2563 \cdot 2)\end{array}$ & $\begin{array}{r}58.5^{*}(49.8 \text { to } \\
69.1)\end{array}$ & $\begin{array}{r}-8 \cdot 8^{*}(-14 \cdot 2 \text { to } \\
-2 \cdot 8)\end{array}$ & $\begin{array}{r}179 \cdot 8(132 \cdot 1 \\
\text { to } 235 \cdot 5)\end{array}$ & $\begin{array}{r}52.5^{*}(44.1 \text { to } \\
62.5)\end{array}$ & 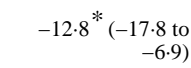 \\
\hline Brain and nervous system cancer & $\begin{array}{r}1193 \cdot 2(1010 \cdot 6 \\
\text { to } 1373 \cdot 3)\end{array}$ & $\begin{array}{r}52.2^{*}(28.6 \text { to } \\
70 \cdot 9)\end{array}$ & $7 \cdot 2(-7 \cdot 2$ to $18 \cdot 9)$ & $\begin{array}{r}121 \cdot 9(87 \cdot 1 \\
\text { to } 165 \cdot 1)\end{array}$ & $\begin{array}{r}55.4 *(34.3 \text { to } \\
71.8)\end{array}$ & $5 \cdot 6(-6.7$ to $15 \cdot 9)$ \\
\hline Thyroid cancer & $\begin{array}{r}2179 \cdot 4(1815 \cdot 3 \\
\text { to } 2509 \cdot 2)\end{array}$ & $\begin{array}{r}100 \cdot 2^{*}(74.8 \text { to } \\
129.9)\end{array}$ & $24 \cdot 4^{*}(9 \cdot 6$ to $41 \cdot 3)$ & $\begin{array}{r}127 \cdot 6(85 \cdot 7 \\
\text { to } 180 \cdot 9)\end{array}$ & $\begin{array}{r}94.9 *(71 \cdot 3 \text { to } \\
122 \cdot 4)\end{array}$ & $19.7^{*}(6.6$ to 35.6$)$ \\
\hline Mesothelioma & $\begin{array}{r}50 \cdot 4(44.2 \text { to } \\
57 \cdot 6)\end{array}$ & $94.7^{*}\left(\begin{array}{r}65.9 \text { to } \\
110.9)\end{array}\right.$ & $12 \cdot 9(-4 \cdot 3$ to $22 \cdot 0)$ & $\begin{array}{r}10 \cdot 8(7.4 \text { to } \\
14 \cdot 4)\end{array}$ & $\begin{array}{r}96.6 *(66.5 \text { to } \\
113.0)\end{array}$ & $14 \cdot 1(-4 \cdot 4$ to $23 \cdot 5)$ \\
\hline Hodgkin's lymphoma & $\begin{array}{r}725 \cdot 3(625 \cdot 0 \text { to } \\
997 \cdot 2)\end{array}$ & $\begin{array}{r}-12.7(-30 \cdot 9 \text { to } \\
59.0)\end{array}$ & $\begin{array}{r}-34 \cdot 7(-46 \cdot 7 \text { to } \\
16.7)\end{array}$ & $\begin{array}{r}57.5(38.7 \text { to } \\
82.1)\end{array}$ & $\begin{array}{r}-12 \cdot 0(-26 \cdot 8 \text { to } \\
56 \cdot 4)\end{array}$ & $\begin{array}{r}-36 \cdot 2(-45 \cdot 7 \text { to } \\
11 \cdot 0)\end{array}$ \\
\hline Non-Hodgkin lymphoma & $\begin{array}{r}2956 \cdot 4(2448 \cdot 0 \\
\text { to } 3253 \cdot 1)\end{array}$ & $\begin{array}{r}121.7^{*}(82.7 \text { to } \\
142.0)\end{array}$ & $\begin{array}{r}38.4 *(12.3 \text { to } \\
50.7)\end{array}$ & $\begin{array}{r}216 \cdot 2(151 \cdot 6 \\
\text { to } 292 \cdot 6)\end{array}$ & 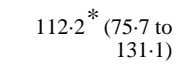 & $31.4 *(6.4$ to 43.3$)$ \\
\hline Multiple myeloma & $\begin{array}{r}427.4(349 \cdot 3 \text { to } \\
528 \cdot 8)\end{array}$ & 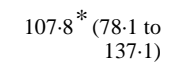 & $19.0^{*}(1.3$ to 36.9$)$ & $\begin{array}{r}86 \cdot 3(59.7 \text { to } \\
114.9)\end{array}$ & $\begin{array}{r}98.6 *(68.9 \text { to } \\
124.1)\end{array}$ & $13.4(-4.5$ to $28 \cdot 6)$ \\
\hline Leukaemia & $\begin{array}{r}2117 \cdot 3(1978.7 \\
\text { to } 2248 \cdot 2)\end{array}$ & 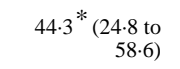 & $6 \cdot 7(-4 \cdot 6$ to $15 \cdot 6)$ & $\begin{array}{r}249 \cdot 6(182.7 \\
\text { to } 320 \cdot 2)\end{array}$ & $\begin{array}{r}52.1 * \begin{array}{r}(38.4 \text { to } \\
63.8)\end{array}\end{array}$ & $4 \cdot 1(-4 \cdot 0$ to $11 \cdot 4)$ \\
\hline Other neoplasms & $\begin{array}{r}6785.0(5938.1 \\
\text { to } 7417.5)\end{array}$ & $\begin{array}{r}185 \cdot 5 *(104.5 \text { to } \\
215 \cdot 4)\end{array}$ & $\begin{array}{r}93.2^{*}(47.0 \text { to } \\
113.0)\end{array}$ & $\begin{array}{r}446 \cdot 8(312.5 \\
\text { to } 596 \cdot 4)\end{array}$ & $\begin{array}{r}172.3 *(102.3 \text { to } \\
199.0)\end{array}$ & $78.3^{*}(39.6$ to 97.0$)$ \\
\hline Cardiovascular diseases & .. &.. &.. & $\begin{array}{r}21177 \cdot 0(14 \\
947 \cdot 8 \text { to } 28 \\
436 \cdot 7)\end{array}$ & $\begin{array}{r}89 \cdot 2 *(69 \cdot 1 \text { to } \\
108 \cdot 7)\end{array}$ & $10 \cdot 3(-1 \cdot 0$ to $21 \cdot 3)$ \\
\hline Rheumatic heart disease & $\begin{array}{r}32903.9(31 \\
608 \cdot 5 \text { to } 34 \\
023 \cdot 7)\end{array}$ & $\begin{array}{r}62.8 *(51.3 \text { to } \\
72.0)\end{array}$ & $13.5^{*}(6.0$ to 19.7$)$ & $\begin{array}{r}1821 \cdot 3 \\
(1211 \cdot 3 \text { to } \\
2530 \cdot 8)\end{array}$ & $\begin{array}{r}60.8 *(47.5 \text { to } \\
73.7)\end{array}$ & $9.5(-0.3$ to $18 \cdot 1)$ \\
\hline Ischaemic heart disease & $\begin{array}{r}92936.7(90 \\
091.9 \text { to } 95 \\
860.8)\end{array}$ & $\begin{array}{r}64.7^{*}(57.0 \text { to } \\
72 \cdot 2)\end{array}$ & $\begin{array}{r}-5.2^{*}(-9.6 \text { to } \\
-1.0)\end{array}$ & $\begin{array}{r}5804 \cdot 1 \\
(4055 \cdot 2 \text { to } \\
7902 \cdot 3)\end{array}$ & $\begin{array}{r}67.5 *(58.8 \text { to } \\
76.6)\end{array}$ & $-4 \cdot 0(-8 \cdot 8$ to $1 \cdot 2)$ \\
\hline Cerebrovascular disease & .. & .. & .. & $\begin{array}{r}3743.6 \\
(2669.9 \text { to } \\
4843.9)\end{array}$ & $\begin{array}{r}83.5 *(75.9 \text { to } \\
93.5)\end{array}$ & $5.0 *(0.5$ to 11.2$)$ \\
\hline Ischaemic stroke & $\begin{array}{r}18305.5(17 \\
767.4 \text { to } 18 \\
920.7)\end{array}$ & $\begin{array}{r}81.5^{*}(72.6 \text { to } \\
92.7)\end{array}$ & $2 \cdot 4(-2 \cdot 8$ to $9 \cdot 1)$ & $\begin{array}{r}2650 \cdot 1 \\
(1875 \cdot 1 \text { to } \\
3492 \cdot 3)\end{array}$ & $\begin{array}{r}81 \cdot 8 * \text { (72.6 to } \\
93.3)\end{array}$ & $3.0(-2 \cdot 2$ to $9 \cdot 9)$ \\
\hline Haemorrhagic stroke & $\begin{array}{r}7363 \cdot 5(7139 \cdot 7 \\
\text { to } 7616 \cdot 1)\end{array}$ & $\begin{array}{r}88.9 *(80.6 \text { to } \\
98.6)\end{array}$ & $10 \cdot 2^{*}(5 \cdot 4$ to $16 \cdot 1)$ & $\begin{array}{r}1093 \cdot 5 \\
(759 \cdot 0 \text { to } \\
1438 \cdot 1)\end{array}$ & $\begin{array}{r}88.5^{*}(79.7 \text { to } \\
99.0)\end{array}$ & $10 \cdot 6^{*}(5 \cdot 6$ to $17 \cdot 1)$ \\
\hline Hypertensive heart disease & $\begin{array}{r}10893 \cdot 7(10 \\
526 \cdot 7 \text { to } 11 \\
246 \cdot 4)\end{array}$ & $\begin{array}{r}112.8^{*}(101.6 \text { to } \\
123.9)\end{array}$ & $\begin{array}{r}19.1 *(12.7 \text { to } \\
25.4)\end{array}$ & $\begin{array}{r}1193.9 \\
(844.9 \text { to } \\
1633.9)\end{array}$ & $\begin{array}{r}113.8 *(102.8 \text { to } \\
124.9)\end{array}$ & $20.0 *$ (13.7 to 26.3$)$ \\
\hline Cardiomyopathy and myocarditis & $\begin{array}{r}7993 \cdot 0(7738 \cdot 3 \\
\text { to } 8269 \cdot 3)\end{array}$ & $\begin{array}{r}85.0 *(77.9 \text { to } \\
93.0)\end{array}$ & $8.5^{*}(4 \cdot 3$ to $13 \cdot 4)$ & $\begin{array}{r}865.7(591.5 \\
\text { to } 1159 \cdot 6)\end{array}$ & $\begin{array}{r}86.8 *(79.0 \text { to } \\
95.1)\end{array}$ & $9.8^{*}$ (5.2 to $\left.14 \cdot 8\right)$ \\
\hline Atrial fibrillation and flutter & $\begin{array}{r}11178 \cdot 6(10 \\
655 \cdot 1 \text { to } 11 \\
683 \cdot 7)\end{array}$ & $\begin{array}{r}63.6 *(53.9 \text { to } \\
71.4)\end{array}$ & $\begin{array}{r}-10 \cdot 3^{*}(-15 \cdot 6 \text { to } \\
-5 \cdot 8)\end{array}$ & $\begin{array}{r}857.8(603.7 \\
\text { to } 1177.4)\end{array}$ & $\begin{array}{r}64.2 *(54.3 \text { to } \\
71.9)\end{array}$ & $\begin{array}{r}-9 \cdot 7^{*}(-15 \cdot 1 \text { to } \\
-5 \cdot 2)\end{array}$ \\
\hline Peripheral vascular disease & $\begin{array}{r}185137.2(172 \\
555.7 \text { to } 196 \\
597.2)\end{array}$ & $\begin{array}{r}78.0 *(59.9 \text { to } \\
91 \cdot 2)\end{array}$ & $-0.1(-9.9$ to 6.7$)$ & $\begin{array}{r}127 \cdot 7(62 \cdot 4 \\
\text { to } 223 \cdot 8)\end{array}$ & $\begin{array}{r}49 \cdot 6 *(31 \cdot 2 \text { to } \\
64 \cdot 3)\end{array}$ & $\begin{array}{r}-19 \cdot 7^{*}(-29.5 \text { to } \\
-12.0)\end{array}$ \\
\hline Endocarditis & $\begin{array}{r}310 \cdot 3(252 \cdot 1 \text { to } \\
361 \cdot 0)\end{array}$ & $\begin{array}{r}71.7^{*}(55.9 \text { to } \\
86.2)\end{array}$ & $4 \cdot 6(-5 \cdot 1$ to $13 \cdot 6)$ & $\begin{array}{r}32.5(21.2 \text { to } \\
46 \cdot 7)\end{array}$ & $\begin{array}{r}77.4 *(59.7 \text { to } \\
96.2)\end{array}$ & $7 \cdot 0(-3.9$ to $18 \cdot 4)$ \\
\hline $\begin{array}{l}\text { Other cardiovascular and circulatory } \\
\text { diseases }\end{array}$ & $\begin{array}{r}95225 \cdot 2(67 \\
433 \cdot 2 \text { to } 121 \\
733.8)\end{array}$ & $\begin{array}{r}130.2{ }^{*}(53.9 \text { to } \\
234 \cdot 7)\end{array}$ & $36 \cdot 4(-8 \cdot 6$ to $98 \cdot 7)$ & $\begin{array}{r}6730 \cdot 4 \\
(3926 \cdot 1 \text { to } 10 \\
097.9)\end{array}$ & 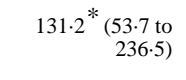 & $37.1(-8.4$ to 99.7$)$ \\
\hline
\end{tabular}




\begin{tabular}{|c|c|c|c|c|c|c|}
\hline & $\begin{array}{l}\text { Prevalent cases } \\
\text { in } 2013(x \\
1000)\end{array}$ & $\begin{array}{l}\text { Percentage } \\
\text { change in } \\
\text { prevalence } \\
\text { from } 1990 \text { to } \\
2013\end{array}$ & $\begin{array}{l}\text { Percentage } \\
\text { change in age- } \\
\text { standardised } \\
\text { prevalence from } \\
1990 \text { to } 2013\end{array}$ & $\begin{array}{l}\text { YLDs in } \\
2013(x \\
1000)\end{array}$ & $\begin{array}{l}\text { Percentage } \\
\text { change in YLDs } \\
\text { from } 1990 \text { to } \\
2013\end{array}$ & $\begin{array}{l}\text { Percentage change } \\
\text { in age- } \\
\text { standardised } \\
\text { YLDs from } 1990 \\
\text { to } 2013\end{array}$ \\
\hline Chronic respiratory diseases & .. & .. & .. & $\begin{array}{r}38618 \cdot 7(26 \\
864 \cdot 7 \text { to } 51 \\
458 \cdot 3)\end{array}$ & $\begin{array}{r}55 \cdot 1^{*}(50 \cdot 3 \text { to } \\
60 \cdot 6)\end{array}$ & $0 \cdot 0(-2 \cdot 5$ to $2 \cdot 8)$ \\
\hline $\begin{array}{l}\text { Chronic obstructive pulmonary } \\
\text { disease }\end{array}$ & $\begin{array}{r}328503 \cdot 6(317 \\
289 \cdot 1 \text { to } 339 \\
461 \cdot 0)\end{array}$ & $\begin{array}{r}65 \cdot 1 *(63 \cdot 8 \text { to } \\
66 \cdot 3)\end{array}$ & $0 \cdot 5(-0 \cdot 2$ to $1 \cdot 2)$ & $\begin{array}{r}26131 \cdot 3(17 \\
785 \cdot 3 \text { to } 35 \\
786 \cdot 9)\end{array}$ & $\begin{array}{r}72.3^{*}(67.4 \text { to } \\
78.6)\end{array}$ & $5.5^{*}(2.7$ to 9.3$)$ \\
\hline Pneumoconiosis & .. & .. & .. & $\begin{array}{r}50 \cdot 8(36 \cdot 0 \text { to } \\
69 \cdot 0)\end{array}$ & $\begin{array}{r}96.9 *(89 \cdot 2 \text { to } \\
103 \cdot 3)\end{array}$ & $17 \cdot 3^{*}$ (12.5 to $\left.21 \cdot 3\right)$ \\
\hline Silicosis & $\begin{array}{r}56 \cdot 2(52 \cdot 6 \text { to } \\
59 \cdot 6)\end{array}$ & $\begin{array}{r}49 \cdot 8^{*}(47 \cdot 3 \text { to } \\
52 \cdot 3)\end{array}$ & $\begin{array}{r}-11.8 *(-13 \cdot 3 \text { to } \\
-10 \cdot 5)\end{array}$ & $\begin{array}{r}10 \cdot 7(6 \cdot 9 \text { to } \\
15 \cdot 1)\end{array}$ & $\begin{array}{r}49 \cdot 9^{*}(47 \cdot 2 \text { to } \\
52 \cdot 3)\end{array}$ & $\begin{array}{r}-11 \cdot 8^{*}(-13 \cdot 3 \text { to } \\
-10 \cdot 5)\end{array}$ \\
\hline Asbestosis & $\begin{array}{r}14 \cdot 8(14 \cdot 0 \text { to } \\
15 \cdot 5)\end{array}$ & $\begin{array}{r}67.5^{*}(64.0 \text { to } \\
71.4)\end{array}$ & $-0 \cdot 7(-2 \cdot 8$ to $1 \cdot 7)$ & $\begin{array}{r}2 \cdot 8(1.8 \text { to } \\
3.9)\end{array}$ & $\begin{array}{r}68 \cdot 1 *(64.6 \text { to } \\
72 \cdot 1)\end{array}$ & $-0 \cdot 4(-2 \cdot 5$ to $2 \cdot 0)$ \\
\hline Coal workers' pneumoconiosis & $\begin{array}{r}36 \cdot 4(34 \cdot 6 \text { to } \\
38 \cdot 1)\end{array}$ & $55 \cdot 1 * \begin{array}{r}(53 \cdot 1 \text { to } \\
56 \cdot 8)\end{array}$ & $\begin{array}{r}-6.9^{*}(-8.0 \text { to } \\
-5.9)\end{array}$ & $\begin{array}{r}6.8(4.6 \text { to } \\
9.8)\end{array}$ & $55 \cdot 1^{*}\left(\begin{array}{r}53.0 \text { to } \\
56.9)\end{array}\right.$ & 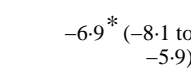 \\
\hline Other pneumoconiosis & $\begin{array}{r}164 \cdot 9(154 \cdot 0 \text { to } \\
174 \cdot 3)\end{array}$ & $\begin{array}{r}144.2 *(137.9 \text { to } \\
150 \cdot 3)\end{array}$ & $\begin{array}{r}47 \cdot 4^{*}(44 \cdot 0 \text { to } \\
51 \cdot 1)\end{array}$ & $\begin{array}{r}30.5(19.9 \text { to } \\
43.9)\end{array}$ & $\begin{array}{r}141 \cdot 8 *(136 \cdot 0 \text { to } \\
148 \cdot 2)\end{array}$ & $45.7^{*}(42.3$ to 49.7$)$ \\
\hline Asthma & $\begin{array}{r}241694 \cdot 7(238 \\
151 \cdot 1 \text { to } 245 \\
464 \cdot 6)\end{array}$ & $\begin{array}{r}32.1 * \text { (29.5 to } \\
34.9)\end{array}$ & $\begin{array}{r}-5.5 *(-7.2 \text { to } \\
-3.5)\end{array}$ & $\begin{array}{r}10595 \cdot 8 \\
(6924 \cdot 6 \text { to } 15 \\
102 \cdot 0)\end{array}$ & $\begin{array}{r}31 \cdot 6 *(28 \cdot 9 \text { to } \\
34 \cdot 6)\end{array}$ & $\begin{array}{r}-5 \cdot 3^{*}(-7 \cdot 2 \text { to } \\
-3 \cdot 2)\end{array}$ \\
\hline $\begin{array}{l}\text { Interstitial lung disease and } \\
\text { pulmonary sarcoidosis }\end{array}$ & $\begin{array}{r}595 \cdot 0(569 \cdot 1 \text { to } \\
622 \cdot 5)\end{array}$ & $\begin{array}{r}69.8^{*}(58.9 \text { to } \\
78.9)\end{array}$ & $5 \cdot 1(-1 \cdot 5$ to $10 \cdot 6)$ & $\begin{array}{r}80 \cdot 7(50 \cdot 7 \text { to } \\
117.9)\end{array}$ & $\begin{array}{r}70 \cdot 9 *(59.5 \text { to } \\
80 \cdot 2)\end{array}$ & $5 \cdot 4(-1.5$ to $11 \cdot 2)$ \\
\hline Other chronic respiratory diseases & .. & .. & .. & $\begin{array}{r}1760 \cdot 0 \\
(1150 \cdot 0 \text { to } \\
2617 \cdot 9)\end{array}$ & $8.4(-4.6$ to $26 \cdot 4)$ & $\begin{array}{r}-32.8 *(-40 \cdot 7 \text { to } \\
-21 \cdot 8)\end{array}$ \\
\hline Cirrhosis & .. & .. & .. & $\begin{array}{r}544 \cdot 6(381 \cdot 1 \\
\text { to } 750 \cdot 4)\end{array}$ & 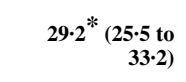 & $\begin{array}{r}-14.9^{*}(-17 \cdot 2 \text { to } \\
-12 \cdot 6)\end{array}$ \\
\hline Cirrhosis due to hepatitis B & $\begin{array}{r}869 \cdot 0(813 \cdot 2 \text { to } \\
923 \cdot 0)\end{array}$ & $\begin{array}{r}21.6 *(11.4 \text { to } \\
30 \cdot 2)\end{array}$ & $\begin{array}{r}-20 \cdot 8^{*}(-27 \cdot 2 \text { to } \\
-15 \cdot 3)\end{array}$ & $\begin{array}{r}143 \cdot 2(98 \cdot 8 \\
\text { to } 197 \cdot 7)\end{array}$ & $\begin{array}{r}20.9 *(9.1 \text { to } \\
31.6)\end{array}$ & $\begin{array}{r}-21 \cdot 0 *(-28 \cdot 1 \text { to } \\
-13 \cdot 9)\end{array}$ \\
\hline Cirrhosis due to hepatitis C & $\begin{array}{r}884.9(837.2 \text { to } \\
947 \cdot 7)\end{array}$ & $\begin{array}{r}60 \cdot 5^{*}(52 \cdot 0 \text { to } \\
74 \cdot 4)\end{array}$ & $1.6(-3.6$ to 10.5$)$ & $\begin{array}{r}144.9(100 \cdot 8 \\
\text { to } 201 \cdot 3)\end{array}$ & $\begin{array}{r}59.9 *(49.8 \text { to } \\
75.3)\end{array}$ & $1 \cdot 6(-4 \cdot 2$ to $11 \cdot 4)$ \\
\hline Cirrhosis due to alcohol use & $\begin{array}{r}801.5(746 \cdot 6 \text { to } \\
864 \cdot 3)\end{array}$ & $\begin{array}{r}10 \cdot 0 *(1 \cdot 1 \text { to } \\
21 \cdot 4)\end{array}$ & $\begin{array}{r}-33 \cdot 4 *(-38.6 \text { to } \\
-27 \cdot 1)\end{array}$ & $\begin{array}{r}131 \cdot 2(89 \cdot 3 \\
\text { to } 180 \cdot 1)\end{array}$ & $\begin{array}{r}10 \cdot 3(-0 \cdot 2 \text { to } \\
22 \cdot 7)\end{array}$ & $\begin{array}{r}-33 \cdot 1^{*}(-39 \cdot 2 \text { to } \\
-26 \cdot 0)\end{array}$ \\
\hline Cirrhosis due to other causes & $\begin{array}{r}742 \cdot 0(693 \cdot 9- \\
789 \cdot 5)\end{array}$ & 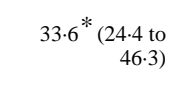 & $3 \cdot 6(-3 \cdot 5$ to $13 \cdot 2)$ & $\begin{array}{r}125 \cdot 3(85 \cdot 6 \\
\text { to } 175 \cdot 1)\end{array}$ & $\begin{array}{r}33 \cdot 3 *(20 \cdot 7 \text { to } \\
50 \cdot 5)\end{array}$ & $3 \cdot 5(-6 \cdot 3$ to $16 \cdot 4)$ \\
\hline Digestive diseases & .. & .. & .. & $\begin{array}{r}8457 \cdot 9 \\
(6066 \cdot 5 \text { to } 11 \\
283.9)\end{array}$ & $\begin{array}{r}24 \cdot 4 *(19 \cdot 9 \text { to } \\
28 \cdot 8)\end{array}$ & $\begin{array}{r}-21 \cdot 9^{*}(-24 \cdot 7 \text { to } \\
-19 \cdot 2)\end{array}$ \\
\hline Peptic ulcer disease & $\begin{array}{r}35515 \cdot 2(33 \\
962 \cdot 5 \text { to } 36 \\
907 \cdot 1)\end{array}$ & $\begin{array}{r}-5.4^{*}(-7.9 \text { to } \\
-2.5)\end{array}$ & $\begin{array}{r}-45 \cdot 8^{*}(-47 \cdot 1 \text { to } \\
-44 \cdot 1)\end{array}$ & $\begin{array}{r}1268 \cdot 6 \\
(873 \cdot 2 \text { to } \\
1774 \cdot 5)\end{array}$ & $-1 \cdot 4(-5 \cdot 9$ to $3 \cdot 7)$ & $\begin{array}{r}-43 \cdot 2^{*}(-45 \cdot 5 \text { to } \\
-40 \cdot 3)\end{array}$ \\
\hline Gastritis and duodenitis & $\begin{array}{r}64799 \cdot 9(63 \\
317 \cdot 5 \text { to } 66 \\
212 \cdot 6)\end{array}$ & $\begin{array}{r}11.4 *(7.8 \text { to } \\
14.9)\end{array}$ & $\begin{array}{r}-26 \cdot 7^{*}(-28 \cdot 7 \text { to } \\
-24 \cdot 7)\end{array}$ & $\begin{array}{r}2384 \cdot 2 \\
(1617 \cdot 0 \text { to } \\
3388 \cdot 7)\end{array}$ & $6.3^{*}(1.7$ to 10.3$)$ & $\begin{array}{r}-27 \cdot 6 *(-30 \cdot 4 \text { to } \\
-25 \cdot 2)\end{array}$ \\
\hline Appendicitis & $\begin{array}{r}621.7(546 \cdot 4 \text { to } \\
699 \cdot 6)\end{array}$ & $\begin{array}{r}17 \cdot 0 *(1 \cdot 0 \text { to } \\
37 \cdot 2)\end{array}$ & $\begin{array}{r}-14 \cdot 6(-25 \cdot 4 \text { to } \\
0 \cdot 0)\end{array}$ & $\begin{array}{r}190 \cdot 2(126 \cdot 9 \\
\text { to } 263 \cdot 1)\end{array}$ & $\begin{array}{r}17 \cdot 4(-1 \cdot 0 \text { to } \\
39 \cdot 2)\end{array}$ & $\begin{array}{r}-13 \cdot 6(-26 \cdot 7 \text { to } \\
1 \cdot 8)\end{array}$ \\
\hline $\begin{array}{l}\text { Paralytic ileus and intestinal } \\
\text { obstruction }\end{array}$ & $\begin{array}{r}114 \cdot 6(111 \cdot 4 \text { to } \\
117 \cdot 6)\end{array}$ & $\begin{array}{r}50 \cdot 4^{*}(44 \cdot 7 \text { to } \\
56 \cdot 3)\end{array}$ & $4 \cdot 6^{*}(1 \cdot 2$ to $8 \cdot 0)$ & $\begin{array}{r}35 \cdot 1(23.8 \text { to } \\
47.5)\end{array}$ & $49 \cdot 2^{*}\left(\begin{array}{r}1 \cdot 2 \text { to } \\
57 \cdot 4)\end{array}\right.$ & $4.7(-0.6$ to 10.6$)$ \\
\hline $\begin{array}{l}\text { Inguinal, femoral, and abdominal } \\
\text { hernia }\end{array}$ & $\begin{array}{r}25393 \cdot 4(24 \\
084.5 \text { to } 26 \\
361 \cdot 0)\end{array}$ & $45 \cdot 7^{*}\left(\begin{array}{r}36 \cdot 0 \text { to } \\
53 \cdot 1)\end{array}\right.$ & $\begin{array}{r}-10 \cdot 5^{*}(-16 \cdot 1 \text { to } \\
-5 \cdot 9)\end{array}$ & $\begin{array}{r}262 \cdot 3(129 \cdot 4 \\
\text { to } 490 \cdot 8)\end{array}$ & $\begin{array}{r}45 \cdot 7^{*}(35 \cdot 8 \text { to } \\
53 \cdot 1)\end{array}$ & 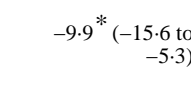 \\
\hline Inflammatory bowel disease & $\begin{array}{r}10575 \cdot 9(10 \\
410 \cdot 1 \text { to } 10 \\
737 \cdot 6)\end{array}$ & $\begin{array}{r}75.7^{*}(72.7 \text { to } \\
78.6)\end{array}$ & $9.6^{*}(7.7$ to 11.4$)$ & $\begin{array}{r}2223 \cdot 2 \\
(1544 \cdot 1 \text { to } \\
3028 \cdot 2)\end{array}$ & $\begin{array}{r}75 \cdot 6^{*}(71.5 \text { to } \\
79 \cdot 5)\end{array}$ & $10 \cdot 1^{*}(7.6$ to 12.5$)$ \\
\hline
\end{tabular}




\begin{tabular}{|c|c|c|c|c|c|c|}
\hline & $\begin{array}{l}\text { Prevalent cases } \\
\text { in } 2013(\times \\
1000)\end{array}$ & $\begin{array}{l}\text { Percentage } \\
\text { change in } \\
\text { prevalence } \\
\text { from } 1990 \text { to } \\
2013\end{array}$ & $\begin{array}{l}\text { Percentage } \\
\text { change in age- } \\
\text { standardised } \\
\text { prevalence from } \\
1990 \text { to } 2013\end{array}$ & $\begin{array}{l}\text { YLDs in } \\
2013(x \\
1000)\end{array}$ & $\begin{array}{l}\text { Percentage } \\
\text { change in YLDs } \\
\text { from } 1990 \text { to } \\
2013\end{array}$ & $\begin{array}{l}\text { Percentage change } \\
\text { in age- } \\
\text { standardised } \\
\text { YLDs from } 1990 \\
\text { to } 2013\end{array}$ \\
\hline Vascular intestinal disorders & $\begin{array}{r}35 \cdot 3(33 \cdot 2 \text { to } \\
38 \cdot 6)\end{array}$ & $\begin{array}{rl}69.9 * & * 58.7 \text { to } \\
81.6)\end{array}$ & $-0.7(-7.6$ to 6.5$)$ & $\begin{array}{r}10 \cdot 8(7.2 \text { to } \\
14.6)\end{array}$ & 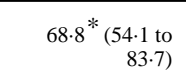 & $-0.2(-9.7$ to 9.8$)$ \\
\hline Gallbladder and biliary diseases & $\begin{array}{r}5893.8(5737.7 \\
\text { to } 6119.0)\end{array}$ & $\begin{array}{r}37.0 *(32.6 \text { to } \\
43.9)\end{array}$ & $\begin{array}{r}-18 \cdot 2 *(-20 \cdot 8 \text { to } \\
-14 \cdot 3)\end{array}$ & $\begin{array}{r}614 \cdot 9(428 \cdot 8 \\
\quad \text { to } 826 \cdot 8)\end{array}$ & $\begin{array}{r}36.9^{*}(31.5 \text { to } \\
43.6)\end{array}$ & $\begin{array}{r}-17.9^{*}(-21.0 \text { to } \\
-14.3)\end{array}$ \\
\hline Pancreatitis & $\begin{array}{r}1972 \cdot 8(1951.7 \\
\text { to } 1997 \cdot 4)\end{array}$ & 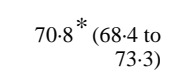 & $6.9^{*}(5.5$ to 8.4$)$ & $\begin{array}{r}579.8(398.9 \\
\text { to } 771 \cdot 6)\end{array}$ & $\begin{array}{r}70.5 *(65.1 \text { to } \\
75.8)\end{array}$ & $7 \cdot 3^{*}(4 \cdot 1$ to 10.5$)$ \\
\hline Other digestive diseases &.. & .. & .. & $\begin{array}{r}888.8(612.7 \\
\text { to } 1228 \cdot 4)\end{array}$ & $5.9(-8.5$ to $17 \cdot 1)$ & $\begin{array}{r}-34 \cdot 2^{*}(-43 \cdot 3 \text { to } \\
-27 \cdot 2)\end{array}$ \\
\hline Neurological disorders &.. &.. &.. & $\begin{array}{r}59360 \cdot 1(41 \\
036 \cdot 0 \text { to } 80 \\
871 \cdot 8)\end{array}$ & $59 \cdot 6 * \begin{array}{r}(54 \cdot 8 \text { to } \\
64 \cdot 5)\end{array}$ & $5.0 *(2.4$ to 7.9$)$ \\
\hline $\begin{array}{l}\text { Alzheimer's disease and other } \\
\text { dementias }\end{array}$ & $\begin{array}{r}53050 \cdot 5(51 \\
663 \cdot 7 \text { to } 54 \\
359 \cdot 8)\end{array}$ & $\begin{array}{r}88.5 *(82.1 \text { to } \\
95.0)\end{array}$ & $-0.7(-4.0$ to 2.8$)$ & $\begin{array}{r}7773 \cdot 6 \\
(5703 \cdot 1 \text { to } \\
9867 \cdot 3)\end{array}$ & $\begin{array}{r}91.8^{*}(85.3 \text { to } \\
98.5)\end{array}$ & $0.0(-3.5$ to 3.8$)$ \\
\hline Parkinson's disease & $\begin{array}{r}5866 \cdot 3(4777 \cdot 2 \\
\text { to } 6950.0)\end{array}$ & $\begin{array}{r}80.9^{*}(77.7 \text { to } \\
83.6)\end{array}$ & $2 \cdot 1^{*}(0.4$ to 3.5$)$ & $\begin{array}{r}694 \cdot 8(468.5 \\
\text { to } 964 \cdot 1)\end{array}$ & $\begin{array}{r}81 \cdot 2^{*}(77 \cdot 3 \text { to } \\
85 \cdot 2)\end{array}$ & $2.7^{*}(0.6$ to 4.6$)$ \\
\hline Epilepsy & $\begin{array}{r}21712 \cdot 0(20 \\
160 \cdot 3 \text { to } 23 \\
108 \cdot 4)\end{array}$ & $\begin{array}{r}31.6^{*}(20.2 \text { to } \\
43.8)\end{array}$ & $-2.5(-10 \cdot 8$ to $6 \cdot 7)$ & $\begin{array}{r}7544 \cdot 2 \\
(5164.1 \text { to } \\
9925.9)\end{array}$ & 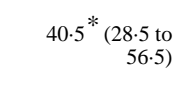 & $4.4(-4.5$ to 15.9$)$ \\
\hline Multiple sclerosis & $\begin{array}{r}2293.6(2238.9 \\
\text { to } 2345 \cdot 6)\end{array}$ & $117 \cdot 2^{*}\left(\begin{array}{r}110 \cdot 0 \text { to } \\
124 \cdot 0)\end{array}\right.$ & $\begin{array}{r}35.4^{*}(30.7 \text { to } \\
39.5)\end{array}$ & $\begin{array}{r}754 \cdot 6(547.6 \\
\text { to } 951.0)\end{array}$ & 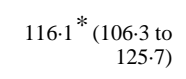 & $35.1{ }^{*}(29.0$ to $41 \cdot 1)$ \\
\hline Migraine & $\begin{array}{r}848366 \cdot 5(831 \\
034 \cdot 6 \text { to } 864 \\
852 \cdot 1)\end{array}$ & $\begin{array}{r}46 \cdot 1^{*}(41 \cdot 4 \text { to } \\
50 \cdot 1)\end{array}$ & $0 \cdot 3(-2 \cdot 8$ to $3 \cdot 0)$ & $\begin{array}{r}28898 \cdot 1(17 \\
585 \cdot 8 \text { to } 42 \\
420 \cdot 1)\end{array}$ & $\begin{array}{r}46 \cdot 1 *(41 \cdot 4 \text { to } \\
50 \cdot 5)\end{array}$ & $0.8(-2.4$ to 3.7$)$ \\
\hline Tension-type headache & $\begin{array}{r}1561446.5(1 \\
537571.4 \text { to } 1 \\
585765 \cdot 8)\end{array}$ & $\begin{array}{r}45.5 *(41.9 \text { to } \\
49.4)\end{array}$ & $0.0(-2 \cdot 4$ to $2 \cdot 6)$ & $\begin{array}{r}2363.2 \\
(1151.9 \text { to } \\
4155.0)\end{array}$ & $\begin{array}{r}45.5 *(41.7 \text { to } \\
49.5)\end{array}$ & $0.3(-2.1$ to 2.9$)$ \\
\hline Medication overuse headache & $\begin{array}{r}62899 \cdot 3(43 \\
143 \cdot 0 \text { to } 80 \\
656 \cdot 0)\end{array}$ & $\begin{array}{r}120 \cdot 2^{*}(109.5 \text { to } \\
133 \cdot 8)\end{array}$ & $42.8 * \begin{array}{r}35.9 \text { to } \\
51.9)\end{array}$ & $\begin{array}{r}9845 \cdot 7 \\
(5777 \cdot 9 \text { to } 15 \\
100 \cdot 3)\end{array}$ & $\begin{array}{r}120.2 *(109 \cdot 3 \text { to } \\
133.7)\end{array}$ & $43.3^{*}$ (36.2 to 52.4$)$ \\
\hline Other neurological disorders & $\begin{array}{r}11 \cdot 6(10 \cdot 0 \text { to } \\
13 \cdot 3)\end{array}$ & $\begin{array}{r}45.9 *(36.0 \text { to } \\
56 \cdot 3)\end{array}$ & $0.9(-5.6$ to 7.9$)$ & $\begin{array}{r}1485 \cdot 8 \\
(1056 \cdot 6 \text { to } \\
1918.6)\end{array}$ & $\begin{array}{r}31.4 *(12.7 \text { to } \\
40.4)\end{array}$ & $\begin{array}{r}-29.6^{*}(-39 \cdot 2 \text { to } \\
-24 \cdot 8)\end{array}$ \\
\hline Mental and substance use disorders & .. & .. &.. & $\begin{array}{r}161811 \cdot 9 \\
(116057 \cdot 8 \text { to } \\
210256 \cdot 0)\end{array}$ & $45 \cdot 0 * \begin{array}{r}(42 \cdot 9 \text { to } \\
47 \cdot 2)\end{array}$ & $1.0 *(0.3$ to 1.9$)$ \\
\hline Schizophrenia & $\begin{array}{r}23600 \cdot 6(22 \\
170 \cdot 6 \text { to } 25 \\
038 \cdot 7)\end{array}$ & $\begin{array}{r}52 \cdot 1 *(50 \cdot 3 \text { to } \\
53 \cdot 6)\end{array}$ & $\begin{array}{r}-1.5 *(-2.4 \text { to } \\
-0.6)\end{array}$ & $\begin{array}{r}15204 \cdot 4(11 \\
169 \cdot 3 \text { to } 18 \\
188 \cdot 6)\end{array}$ & $\begin{array}{r}52 \cdot 1 *(50 \cdot 3 \text { to } \\
54 \cdot 1)\end{array}$ & $-1 \cdot 1(-2 \cdot 2$ to $0 \cdot 1)$ \\
\hline Alcohol use disorders & $\begin{array}{r}76896.6(73 \\
454.0 \text { to } 80 \\
491.9)\end{array}$ & $\begin{array}{r}34 \cdot 4^{*}(32.3 \text { to } \\
36 \cdot 4)\end{array}$ & $\begin{array}{r}-7.6 *(-8.8 \text { to } \\
-6.5)\end{array}$ & $\begin{array}{r}7653 \cdot 6 \\
(5150 \cdot 7 \text { to } 10 \\
952 \cdot 3)\end{array}$ & $\begin{array}{r}34.3 *(32.0 \text { to } \\
36 \cdot 6)\end{array}$ & $\begin{array}{r}-7.5^{*}(-8 \cdot 8 \text { to } \\
-6 \cdot 1)\end{array}$ \\
\hline Drug use disorders & .. & .. & .. & $\begin{array}{r}12222 \cdot 4 \\
(8513 \cdot 1 \text { to } 16 \\
172 \cdot 8)\end{array}$ & $\begin{array}{r}41 \cdot 3 *(36 \cdot 3 \text { to } \\
46 \cdot 3)\end{array}$ & $0.6(-2 \cdot 4$ to $3 \cdot 8)$ \\
\hline Opioid use disorders & $\begin{array}{r}14071 \cdot 8(11 \\
103 \cdot 0 \text { to } 181 \\
39 \cdot 2)\end{array}$ & $\begin{array}{r}58.9^{*}(54.4 \text { to } \\
62.8)\end{array}$ & $6 \cdot 4^{*}(3 \cdot 0$ to $9 \cdot 5)$ & $\begin{array}{r}5849.5 \\
(3907.9 \text { to } \\
8184.0)\end{array}$ & $\begin{array}{r}59.1 *(54.0 \text { to } \\
63.6)\end{array}$ & $6 \cdot 7^{*}(3 \cdot 2$ to $10 \cdot 2)$ \\
\hline Cocaine use disorders & $\begin{array}{r}7384 \cdot 6(7216 \cdot 3 \\
\text { to } 7549 \cdot 3)\end{array}$ & $\begin{array}{r}32.1 *(28.4 \text { to } \\
35.8)\end{array}$ & $\begin{array}{r}-5.9 *(-8.5 \text { to } \\
-3.3)\end{array}$ & $\begin{array}{r}1012.9 \\
(666 \cdot 9 \text { to } \\
1430 \cdot 2)\end{array}$ & 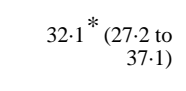 & $\begin{array}{r}-5.7^{*}(-9 \cdot 2 \text { to } \\
-2 \cdot 3)\end{array}$ \\
\hline Amphetamine use disorders & $\begin{array}{r}14922.9(14 \\
490 \cdot 6 \text { to } 15 \\
370 \cdot 7)\end{array}$ & 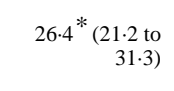 & 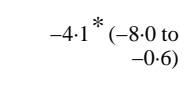 & $\begin{array}{r}1961 \cdot 0 \\
(1233.0 \text { to } \\
2832.9)\end{array}$ & $26.6 * \begin{array}{r}(20 \cdot 8 \text { to } \\
32 \cdot 2)\end{array}$ & $-3 \cdot 8(-8 \cdot 1$ to 0.3$)$ \\
\hline Cannabis use disorders & $\begin{array}{r}13625 \cdot 0(12 \\
429 \cdot 2 \text { to } 14 \\
938.0)\end{array}$ & $\begin{array}{r}22 \cdot 4^{*}(20 \cdot 6 \text { to } \\
24 \cdot 0)\end{array}$ & $\begin{array}{r}-6.5^{*}(-7.6 \text { to } \\
-5.5)\end{array}$ & $\begin{array}{r}395.6(261.2 \\
\text { to } 576 \cdot 2)\end{array}$ & $\begin{array}{r}22 \cdot 4 *(19 \cdot 0 \text { to } \\
26 \cdot 1)\end{array}$ & $\begin{array}{r}-6.4^{*}(-8.9 \text { to } \\
-3.7)\end{array}$ \\
\hline
\end{tabular}




\begin{tabular}{|c|c|c|c|c|c|c|}
\hline & $\begin{array}{l}\text { Prevalent cases } \\
\text { in } 2013(\times \\
1000)\end{array}$ & $\begin{array}{l}\text { Percentage } \\
\text { change in } \\
\text { prevalence } \\
\text { from } 1990 \text { to } \\
2013\end{array}$ & $\begin{array}{l}\text { Percentage } \\
\text { change in age- } \\
\text { standardised } \\
\text { prevalence from } \\
1990 \text { to } 2013\end{array}$ & $\begin{array}{l}\text { YLDs in } \\
2013(\times \\
1000)\end{array}$ & $\begin{array}{l}\text { Percentage } \\
\text { change in YLDs } \\
\text { from } 1990 \text { to } \\
2013\end{array}$ & $\begin{array}{l}\text { Percentage change } \\
\text { in age- } \\
\text { standardised } \\
\text { YLDs from } 1990 \\
\text { to } 2013\end{array}$ \\
\hline Other drug use disorders & .. & .. & .. & $\begin{array}{r}3003.3 \\
(1998.9 \text { to } \\
4164.5)\end{array}$ & $\begin{array}{r}28.5 *(21.5 \text { to } \\
36.5)\end{array}$ & $-4.4(-9.7$ to 1.4$)$ \\
\hline Depressive disorders & .. & .. & .. & $\begin{array}{r}61632 \cdot 8(41 \\
353 \cdot 8 \text { to } 85 \\
621 \cdot 4)\end{array}$ & $\begin{array}{r}53 \cdot 6 * \text { (49.6 to } \\
58 \cdot 4)\end{array}$ & $4.0^{*}(2 \cdot 2$ to $5 \cdot 6)$ \\
\hline Major depressive disorder & $\begin{array}{r}253314.2(208 \\
457 \cdot 2 \text { to } 299 \\
691 \cdot 3)\end{array}$ & $\begin{array}{r}53.4^{*}(49.0 \text { to } \\
58.9)\end{array}$ & $4 \cdot 2^{*}(2 \cdot 4$ to $6 \cdot 2)$ & $\begin{array}{r}51783.9(33 \\
888.2 \text { to } 73 \\
665 \cdot 8)\end{array}$ & $\begin{array}{r}53.4 *(48.8 \text { to } \\
59.2)\end{array}$ & $4.7^{*}(2.7$ to 6.7$)$ \\
\hline Dysthymia & $\begin{array}{r}102409 \cdot 6(91 \\
246 \cdot 2 \text { to } 113 \\
441 \cdot 2)\end{array}$ & $\begin{array}{r}54.6^{*}(52.4 \text { to } \\
57 \cdot 2)\end{array}$ & $-0 \cdot 3(-1 \cdot 2$ to $0 \cdot 7)$ & $\begin{array}{r}9848.9 \\
(6586 \cdot 6 \text { to } 14 \\
166 \cdot 0)\end{array}$ & $54 \cdot 6 * \begin{array}{r}(52.1 \text { to } \\
57 \cdot 2)\end{array}$ & $0 \cdot 1(-1.0$ to $1 \cdot 2)$ \\
\hline Bipolar disorder & $\begin{array}{r}48778.4(43 \\
498.9 \text { to } 54 \\
371.2)\end{array}$ & $\begin{array}{r}49 \cdot 1^{*}(46 \cdot 7 \text { to } \\
52 \cdot 2)\end{array}$ & $0 \cdot 9(-0 \cdot 4$ to $2 \cdot 4)$ & $\begin{array}{r}9911 \cdot 1 \\
(6260 \cdot 6 \text { to } 14 \\
791 \cdot 0)\end{array}$ & $\begin{array}{r}49 \cdot 2 *(46 \cdot 4 \text { to } \\
52 \cdot 5)\end{array}$ & $1.3(-0.4$ to 2.9$)$ \\
\hline Anxiety disorders & $\begin{array}{r}265610 \cdot 1(213 \\
015 \cdot 1 \text { to } 318 \\
817 \cdot 0)\end{array}$ & $\begin{array}{r}42.4^{*}(36.6 \text { to } \\
46.8)\end{array}$ & $-0.5(-1.7$ to 0.8$)$ & $\begin{array}{r}24355 \cdot 8(16 \\
148 \cdot 6 \text { to } 35 \\
139 \cdot 0)\end{array}$ & $\begin{array}{r}42 \cdot 1^{*}(36.4 \text { to } \\
46 \cdot 5)\end{array}$ & $-0.2(-1.6$ to 1.3$)$ \\
\hline Eating disorders & .. & .. & .. & $\begin{array}{r}1820 \cdot 0 \\
(1154 \cdot 5 \text { to } \\
2720 \cdot 3)\end{array}$ & $\begin{array}{r}27.6 *(23.7 \text { to } \\
32 \cdot 1)\end{array}$ & $-1.2(-4.2$ to $1 \cdot 7)$ \\
\hline Anorexia nervosa & $\begin{array}{r}2063 \cdot 6(1683 \cdot 5 \\
\text { to } 2535 \cdot 0)\end{array}$ & $\begin{array}{r}35.4^{*}(31.1 \text { to } \\
40 \cdot 7)\end{array}$ & $5.5^{*}(2 \cdot 2$ to $9 \cdot 2)$ & $\begin{array}{r}440 \cdot 4(285 \cdot 5 \\
\text { to } 648 \cdot 3)\end{array}$ & $\begin{array}{r}35.9 *(30.2 \text { to } \\
41 \cdot 8)\end{array}$ & $6.0 *(1.7$ to 10.3$)$ \\
\hline Bulimia nervosa & $\begin{array}{r}6537.9(4917.2 \\
\text { to } 8857 \cdot 6)\end{array}$ & 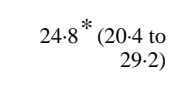 & $\begin{array}{r}-3 \cdot 7^{*}(-6 \cdot 9 \text { to } \\
-0 \cdot 8)\end{array}$ & $\begin{array}{r}1379 \cdot 7 \\
(850 \cdot 7 \text { to } \\
2136 \cdot 6)\end{array}$ & 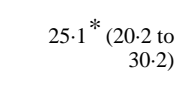 & $-3 \cdot 4(-7 \cdot 1$ to $0 \cdot 1)$ \\
\hline Autistic spectrum disorders & .. & .. & .. & $\begin{array}{r}8449.0 \\
\text { (5888.1 to } 11 \\
458 \cdot 7)\end{array}$ & $\begin{array}{r}33.8 *(32.7 \text { to } \\
34.9)\end{array}$ & $0.7(-0.0$ to 1.5$)$ \\
\hline Autism & $\begin{array}{r}21716 \cdot 7(20 \\
731 \cdot 8 \text { to } 22 \\
713 \cdot 5)\end{array}$ & $\begin{array}{r}34 \cdot 1^{*}(33 \cdot 5 \text { to } \\
34 \cdot 8)\end{array}$ & $0 \cdot 3^{*}(0 \cdot 1$ to $0 \cdot 6)$ & $\begin{array}{r}5345.0 \\
(3583.6 \text { to } \\
7309.9)\end{array}$ & $\begin{array}{r}33.9 *(32.4 \text { to } \\
35.5)\end{array}$ & $0.7(-0.3$ to $1 \cdot 8)$ \\
\hline Asperger's syndrome & $\begin{array}{r}31100 \cdot 1(29 \\
251 \cdot 7 \text { to } 32 \\
905 \cdot 1)\end{array}$ & $\begin{array}{r}33.7^{*}(32.9 \text { to } \\
34.5)\end{array}$ & $0 \cdot 4^{*}(0 \cdot 3$ to $0 \cdot 5)$ & $\begin{array}{r}3104 \cdot 0 \\
(2169 \cdot 6 \text { to } \\
4325 \cdot 0)\end{array}$ & $\begin{array}{r}33.6 * \text { (32.3 to } \\
35.0)\end{array}$ & $0.7(-0.1$ to 1.5$)$ \\
\hline $\begin{array}{l}\text { Attention-deficit or hyperactivity } \\
\text { disorder }\end{array}$ & $\begin{array}{r}39343 \cdot 5(36 \\
574.2 \text { to } 42 \\
093 \cdot 6)\end{array}$ & $\begin{array}{r}13 \cdot 8^{*}(12 \cdot 8 \text { to } \\
14 \cdot 9)\end{array}$ & $-0 \cdot 5(-1 \cdot 3$ to 0.5$)$ & $\begin{array}{r}479.9(287.4 \\
\text { to } 745.8)\end{array}$ & $\begin{array}{r}13 \cdot 9^{*}(12 \cdot 1 \text { to } \\
16 \cdot 0)\end{array}$ & $-0.3(-1.9$ to 1.5$)$ \\
\hline Conduct disorder & $\begin{array}{r}51109 \cdot 7(48 \\
006 \cdot 9 \text { to } 54 \\
557 \cdot 0)\end{array}$ & $\begin{array}{r}16 \cdot 3 *(15 \cdot 4 \text { to } \\
17 \cdot 2)\end{array}$ & $2 \cdot 2^{*}(1 \cdot 4$ to $3 \cdot 1)$ & $\begin{array}{r}6159 \cdot 0 \\
(3868 \cdot 2 \text { to } \\
8911 \cdot 6)\end{array}$ & $\begin{array}{r}16 \cdot 5 *(15 \cdot 0 \text { to } \\
18 \cdot 0)\end{array}$ & $2.4^{*}(1.1$ to 3.8$)$ \\
\hline Idiopathic intellectual disability & $\begin{array}{r}94672 \cdot 8(75 \\
906 \cdot 5 \text { to } 116 \\
664 \cdot 7)\end{array}$ & $22.6^{*}\left(\begin{array}{r}12.7 \text { to } \\
35 \cdot 8)\end{array}\right.$ & $-5 \cdot 8(-13 \cdot 5$ to $4 \cdot 4)$ & $\begin{array}{r}4666 \cdot 7 \\
(3084 \cdot 8 \text { to } \\
6640 \cdot 0)\end{array}$ & $\begin{array}{r}22 \cdot 0 * \text { (12.1 to } \\
35 \cdot 0)\end{array}$ & $-6 \cdot 1(-13 \cdot 9$ to $3 \cdot 8)$ \\
\hline $\begin{array}{l}\text { Other mental and substance use } \\
\text { disorders }\end{array}$ & $\begin{array}{r}124042.9(116 \\
741.4 \text { to } 131 \\
001 \cdot 8)\end{array}$ & $\begin{array}{r}52.3^{*}(50.9 \text { to } \\
53 \cdot 8)\end{array}$ & $0 \cdot 2^{*}(0 \cdot 1$ to $0 \cdot 3)$ & $\begin{array}{r}9257 \cdot 2 \\
(6277 \cdot 9 \text { to } \\
12411 \cdot 5)\end{array}$ & $\begin{array}{r}52 \cdot 3 *(50 \cdot 5 \text { to } \\
54 \cdot 1)\end{array}$ & $0 \cdot 6(-0 \cdot 1$ to $1 \cdot 3)$ \\
\hline $\begin{array}{l}\text { Diabetes, urogenital, blood, and } \\
\text { endocrine diseases }\end{array}$ & .. & .. & .. & $\begin{array}{r}65561 \cdot 4(46 \\
201 \cdot 5 \text { to } 87 \\
834 \cdot 5)\end{array}$ & $\begin{array}{r}72 \cdot 7^{*}(69 \cdot 2 \text { to } \\
76 \cdot 6)\end{array}$ & $\begin{array}{r}14 \cdot 1 *(11 \cdot 9 \text { to } \\
16 \cdot 4)\end{array}$ \\
\hline Diabetes mellitus & $\begin{array}{r}409967 \cdot 0(381 \\
806 \cdot 3 \text { to } 432 \\
038 \cdot 4)\end{array}$ & $\begin{array}{r}132.9^{*}(123.7 \text { to } \\
142.4)\end{array}$ & $\begin{array}{r}44.8 *(38.5 \text { to } \\
51 \cdot 3)\end{array}$ & $\begin{array}{r}29518 \cdot 1(20 \\
419 \cdot 1 \text { to } 40 \\
169 \cdot 3)\end{array}$ & $\begin{array}{r}135 \cdot 7^{*}(127 \cdot 2 \text { to } \\
143 \cdot 7)\end{array}$ & $43.4^{*}(37.9$ to 48.5$)$ \\
\hline Acute glomerulonephritis & $\begin{array}{r}37 \cdot 7(35 \cdot 1 \text { to } \\
40 \cdot 5)\end{array}$ & $\begin{array}{r}-3 \cdot 4^{*}(-6 \cdot 0 \text { to } \\
-1 \cdot 2)\end{array}$ & $\begin{array}{r}-22 \cdot 2^{*}(-24 \cdot 2 \text { to } \\
-20 \cdot 6)\end{array}$ & $\begin{array}{r}1.9(1.2 \text { to } \\
2 \cdot 8)\end{array}$ & $\begin{array}{r}-3 \cdot 3^{*}(-6 \cdot 0 \text { to } \\
-1 \cdot 1)\end{array}$ & $\begin{array}{r}-22 \cdot 2^{*}(-24 \cdot 2 \text { to } \\
-20 \cdot 6)\end{array}$ \\
\hline Chronic kidney disease & .. & .. & .. & $\begin{array}{r}12347 \cdot 3 \\
(9101 \cdot 6 \text { to } 15 \\
814.9)\end{array}$ & $\begin{array}{r}49.5 *(43.4 \text { to } \\
53.9)\end{array}$ & $\begin{array}{r}-2 \cdot 8^{*}(-6.4 \text { to } \\
-0 \cdot 4)\end{array}$ \\
\hline $\begin{array}{l}\text { Chronic kidney disease due to } \\
\text { diabetes mellitus }\end{array}$ & $\begin{array}{r}88710 \cdot 9(71 \\
150 \cdot 1 \text { to } 111 \\
417 \cdot 2)\end{array}$ & $\begin{array}{r}82.4^{*}(58.6 \text { to } \\
96 \cdot 8)\end{array}$ & $11.9(-3.6$ to 21.2$)$ & $\begin{array}{r}2491 \cdot 8 \\
(1802 \cdot 9 \text { to } \\
3220 \cdot 7)\end{array}$ & $\begin{array}{r}80.5 *(65 \cdot 7 \text { to } \\
92 \cdot 6)\end{array}$ & $10 \cdot 6^{*}(2 \cdot 0$ to $17 \cdot 7)$ \\
\hline
\end{tabular}

Lancet. Author manuscript; available in PMC 2015 September 07. 


\begin{tabular}{|c|c|c|c|c|c|c|}
\hline & $\begin{array}{l}\text { Prevalent cases } \\
\text { in } 2013(\times \\
1000)\end{array}$ & $\begin{array}{l}\text { Percentage } \\
\text { change in } \\
\text { prevalence } \\
\text { from } 1990 \text { to } \\
2013\end{array}$ & $\begin{array}{l}\text { Percentage } \\
\text { change in age- } \\
\text { standardised } \\
\text { prevalence from } \\
1990 \text { to } 2013\end{array}$ & $\begin{array}{l}\text { YLDs in } \\
2013(x \\
1000)\end{array}$ & $\begin{array}{l}\text { Percentage } \\
\text { change in YLDs } \\
\text { from } 1990 \text { to } \\
2013\end{array}$ & $\begin{array}{l}\text { Percentage change } \\
\text { in age- } \\
\text { standardised } \\
\text { YLDs from } 1990 \\
\text { to } 2013\end{array}$ \\
\hline $\begin{array}{l}\text { Chronic kidney disease due to } \\
\text { hypertension }\end{array}$ & $\begin{array}{r}101253 \cdot 4(81 \\
410 \cdot 4 \text { to } 129 \\
993 \cdot 0)\end{array}$ & $\begin{array}{r}26 \cdot 8^{*}(18 \cdot 3 \text { to } \\
34 \cdot 9)\end{array}$ & $\begin{array}{r}-10 \cdot 7^{*}(-17 \cdot 6 \text { to } \\
-5 \cdot 3)\end{array}$ & $\begin{array}{r}2635 \cdot 3 \\
(1915 \cdot 3 \text { to } \\
3415 \cdot 5)\end{array}$ & $23 \cdot 1 *\left(\begin{array}{r}16 \cdot 3 \text { to } \\
33 \cdot 2)\end{array}\right.$ & $\begin{array}{r}-22 \cdot 4 *(-26 \cdot 9 \text { to } \\
-16 \cdot 3)\end{array}$ \\
\hline $\begin{array}{l}\text { Chronic kidney disease due to } \\
\text { glomerulonephritis }\end{array}$ & $\begin{array}{r}108860 \cdot 9(88 \\
330 \cdot 3 \text { to } 135 \\
481 \cdot 9)\end{array}$ & $\begin{array}{r}32.7^{*}(17 \cdot 3 \text { to } \\
41 \cdot 3)\end{array}$ & $\begin{array}{r}-13 \cdot 5^{*}(-25 \cdot 6 \text { to } \\
-6 \cdot 6)\end{array}$ & $\begin{array}{r}2495 \cdot 9 \\
(1809 \cdot 0 \text { to } \\
3252 \cdot 0)\end{array}$ & $\begin{array}{r}33.9 *(25 \cdot 6 \text { to } \\
43 \cdot 3)\end{array}$ & $\begin{array}{r}-6.9 *(-11.9 \text { to } \\
-0.5)\end{array}$ \\
\hline $\begin{array}{l}\text { Chronic kidney disease due to } \\
\text { other causes }\end{array}$ & $\begin{array}{r}173090 \cdot 7(142 \\
395 \cdot 9 \text { to } 213 \\
010 \cdot 4)\end{array}$ & $\begin{array}{r}53.9^{*}(43.5 \text { to } \\
63.6)\end{array}$ & $3 \cdot 1(-5 \cdot 9$ to $10 \cdot 1)$ & $\begin{array}{r}4724 \cdot 3 \\
(3503 \cdot 0 \text { to } \\
6063 \cdot 2)\end{array}$ & $\begin{array}{r}63.4 *(55.9 \text { to } \\
71.9)\end{array}$ & $6.9^{*}(0.9$ to 12.7$)$ \\
\hline Urinary diseases and male infertility & .. & .. & .. & $\begin{array}{r}4880 \cdot 3 \\
(3212 \cdot 9 \text { to } \\
7017 \cdot 1)\end{array}$ & $\begin{array}{r}83.8 *(77 \cdot 8 \text { to } \\
91 \cdot 1)\end{array}$ & $8 \cdot 5^{*}(5 \cdot 1$ to $12 \cdot 4)$ \\
\hline $\begin{array}{l}\text { Interstitial nephritis and urinary } \\
\text { tract infections }\end{array}$ & $\begin{array}{r}1753 \cdot 7(1730 \cdot 2 \\
\text { to } 1775 \cdot 3)\end{array}$ & $\begin{array}{r}67.5^{*}(64.6 \text { to } \\
70 \cdot 9)\end{array}$ & $\begin{array}{r}16 \cdot 2^{*}(14 \cdot 3 \text { to } \\
18 \cdot 4)\end{array}$ & $\begin{array}{r}58 \cdot 1(36 \cdot 4 \text { to } \\
85 \cdot 6)\end{array}$ & $\begin{array}{r}66.8 *(59.9 \text { to } \\
74 \cdot 7)\end{array}$ & $16.4^{*}(11.8$ to 21.3$)$ \\
\hline Urolithiasis & $\begin{array}{r}80622.5(61 \\
562 \cdot 7 \text { to } 103 \\
069 \cdot 9)\end{array}$ & $\begin{array}{r}101.9^{*}(89.6 \text { to } \\
113 \cdot 3)\end{array}$ & $\begin{array}{r}20 \cdot 0 *(12.9 \text { to } \\
26 \cdot 1)\end{array}$ & $\begin{array}{r}661 \cdot 8(411 \cdot 4 \\
\text { to } 984 \cdot 0)\end{array}$ & $\begin{array}{r}95 \cdot 8 *(83.0 \text { to } \\
109 \cdot 3)\end{array}$ & $20 \cdot 4^{*}(13.0$ to $27 \cdot 6)$ \\
\hline Benign prostatic hyperplasia & $\begin{array}{r}99148 \cdot 4(97 \\
356 \cdot 1 \text { to } 100 \\
911 \cdot 6)\end{array}$ & $\begin{array}{r}79.3^{*}(75.4 \text { to } \\
83 \cdot 7)\end{array}$ & $3.0 *(0 \cdot 8$ to $5 \cdot 6)$ & $\begin{array}{r}3552 \cdot 9 \\
(2316 \cdot 5 \text { to } \\
4993 \cdot 7)\end{array}$ & $\begin{array}{r}80.0 *(75.9 \text { to } \\
84.5)\end{array}$ & $3.6^{*}(1.3$ to $6 \cdot 3)$ \\
\hline $\begin{array}{l}\text { Male infertility due to other } \\
\text { causes }\end{array}$ & $\begin{array}{r}39276 \cdot 2(36 \\
950 \cdot 6 \text { to } 41 \\
618 \cdot 7)\end{array}$ & $\begin{array}{r}44 \cdot 0 *(33 \cdot 3 \text { to } \\
55 \cdot 3)\end{array}$ & $2.9(-4.8$ to $11 \cdot 0)$ & $\begin{array}{r}258 \cdot 6(111 \cdot 8 \\
\text { to } 531 \cdot 4)\end{array}$ & $\begin{array}{r}43 \cdot 6^{*}(32 \cdot 3 \text { to } \\
56 \cdot 6)\end{array}$ & $3 \cdot 0(-5 \cdot 1$ to $12 \cdot 0)$ \\
\hline Other urinary diseases & .. & .. & .. & $\begin{array}{r}348.9(175.8 \\
\text { to } 544.0)\end{array}$ & $\begin{array}{r}195 \cdot 7^{*}(94.2 \text { to } \\
241 \cdot 5)\end{array}$ & 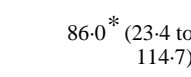 \\
\hline Gynaecological diseases & .. & .. & .. & $\begin{array}{r}9131.4 \\
(5982.5 \text { to } 13 \\
595 \cdot 8)\end{array}$ & $\begin{array}{r}44 \cdot 5 *(40 \cdot 3 \text { to } \\
49 \cdot 3)\end{array}$ & $\begin{array}{r}-3 \cdot 5^{*}(-6 \cdot 1 \text { to } \\
-0 \cdot 5)\end{array}$ \\
\hline Uterine fibroids & $\begin{array}{r}171005 \cdot 4(158 \\
155 \cdot 9 \text { to } 182 \\
513.5)\end{array}$ & $\begin{array}{r}62.5^{*}(61.2 \text { to } \\
63.8)\end{array}$ & $\begin{array}{r}-1.8^{*}(-2.3 \text { to } \\
-1 \cdot 2)\end{array}$ & $\begin{array}{r}2164 \cdot 7 \\
(1242 \cdot 3 \text { to } \\
3677 \cdot 9)\end{array}$ & $\begin{array}{r}36 \cdot 6^{*}(29 \cdot 4 \text { to } \\
43 \cdot 6)\end{array}$ & $\begin{array}{r}-14 \cdot 3^{*}(-18 \cdot 3 \text { to } \\
-10 \cdot 6)\end{array}$ \\
\hline Polycystic ovarian syndrome & $\begin{array}{r}122310 \cdot 6(119 \\
572 \cdot 7 \text { to } 124 \\
929 \cdot 3)\end{array}$ & $\begin{array}{r}51 \cdot 1^{*}(46 \cdot 6 \text { to } \\
55 \cdot 6)\end{array}$ & $2 \cdot 3(-0 \cdot 6$ to $5 \cdot 2)$ & $\begin{array}{r}1191 \cdot 2 \\
(562 \cdot 3 \text { to } \\
2227 \cdot 4)\end{array}$ & 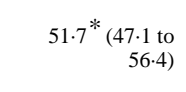 & $3 \cdot 0^{*}(0 \cdot 1$ to $6 \cdot 1)$ \\
\hline $\begin{array}{l}\text { Female infertility due to other } \\
\text { causes }\end{array}$ & $\begin{array}{r}34678 \cdot 6(31 \\
103 \cdot 9 \text { to } 38 \\
009 \cdot 3)\end{array}$ & $\begin{array}{r}58 \cdot 3^{*}(44 \cdot 8 \text { to } \\
72 \cdot 4)\end{array}$ & $10 \cdot 7^{*}(1 \cdot 7$ to $20 \cdot 3)$ & $\begin{array}{r}191 \cdot 9(75 \cdot 2 \\
\text { to } 399 \cdot 1)\end{array}$ & $\begin{array}{r}56 \cdot 9 *(42.4 \text { to } \\
71 \cdot 6)\end{array}$ & $11.0^{*}(1.0$ to 21.0$)$ \\
\hline Endometriosis & $\begin{array}{r}14661 \cdot 2(14 \\
230 \cdot 8 \text { to } 15 \\
094 \cdot 4)\end{array}$ & $\begin{array}{r}47.9 *(41.9 \text { to } \\
53.5)\end{array}$ & $-1 \cdot 6(-5 \cdot 6$ to $2 \cdot 1)$ & $\begin{array}{r}1359 \cdot 6 \\
(906 \cdot 4 \text { to } \\
1861 \cdot 5)\end{array}$ & $\begin{array}{r}48 \cdot 1 *(42 \cdot 1 \text { to } \\
54 \cdot 3)\end{array}$ & $-1 \cdot 3(-5 \cdot 3$ to $2 \cdot 8)$ \\
\hline Genital prolapse & $\begin{array}{r}343707 \cdot 8(336 \\
958 \cdot 8 \text { to } 350 \\
327 \cdot 4)\end{array}$ & $\begin{array}{r}57.0 *(53.0 \text { to } \\
61.5)\end{array}$ & $-0.9(-3.3$ to 1.7$)$ & $\begin{array}{r}1094 \cdot 2 \\
(534 \cdot 5 \text { to } \\
2041 \cdot 0)\end{array}$ & $\begin{array}{r}57.1 *(52.9 \text { to } \\
61.7)\end{array}$ & $-0 \cdot 7(-3 \cdot 3$ to $2 \cdot 1)$ \\
\hline Premenstrual syndrome & $\begin{array}{r}302731 \cdot 7(284 \\
373 \cdot 1 \text { to } 321 \\
829 \cdot 6)\end{array}$ & $\begin{array}{r}53 \cdot 8^{*}(40 \cdot 3 \text { to } \\
67 \cdot 9)\end{array}$ & $9 \cdot 2(-0 \cdot 1$ to $19 \cdot 4)$ & $\begin{array}{r}2548 \cdot 6 \\
(1581 \cdot 3 \text { to } \\
3777 \cdot 0)\end{array}$ & $\begin{array}{r}54.0 *(40 \cdot 7 \text { to } \\
67.9)\end{array}$ & $9.5^{*}(0.1$ to 19.5$)$ \\
\hline Other gynaecological diseases & $\begin{array}{r}20758.0(19 \\
699.4 \text { to } 21 \\
792 \cdot 8)\end{array}$ & $\begin{array}{r}15 \cdot 7^{*}(6 \cdot 9 \text { to } \\
26 \cdot 4)\end{array}$ & $\begin{array}{r}-19 \cdot 3 *(-25 \cdot 6 \text { to } \\
-11 \cdot 8)\end{array}$ & $\begin{array}{r}581 \cdot 3(395 \cdot 7 \\
\text { to } 826 \cdot 6)\end{array}$ & $4 \cdot 2(-4 \cdot 6$ to $21 \cdot 3)$ & $\begin{array}{r}-27.2 *(-33 \cdot 5 \text { to } \\
-14.7)\end{array}$ \\
\hline $\begin{array}{l}\text { Haemoglobinopathies and } \\
\text { haemolytic anaemias }\end{array}$ & .. & .. & .. & $\begin{array}{r}7071 \cdot 9 \\
(4730 \cdot 8 \text { to } \\
10107 \cdot 0)\end{array}$ & $\begin{array}{r}23 \cdot 2^{*}(20 \cdot 7 \text { to } \\
26 \cdot 5)\end{array}$ & $-2 \cdot 3(-4 \cdot 2$ to $0 \cdot 3)$ \\
\hline Thalassaemias & $\begin{array}{r}766.7(691.8 \text { to } \\
849 \cdot 8)\end{array}$ & $\begin{array}{r}11.6 *(8.5 \text { to } \\
19 \cdot 1)\end{array}$ & $-2 \cdot 5(-5 \cdot 1$ to $3 \cdot 8)$ & $\begin{array}{r}61.9(42.1 \text { to } \\
88.0)\end{array}$ & $\begin{array}{r}10.2 *(1.8 \text { to } \\
22 \cdot 1)\end{array}$ & $-4 \cdot 2(-11 \cdot 3$ to $5 \cdot 8)$ \\
\hline Thalassaemia trait & $\begin{array}{r}207561 \cdot 8(192 \\
411 \cdot 3 \text { to } 228 \\
437 \cdot 6)\end{array}$ & $\begin{array}{r}37.3 *(34.7 \text { to } \\
39.6)\end{array}$ & $2.7^{*}(0.5$ to 4.5$)$ & $\begin{array}{r}3769.6 \\
(2508.9 \text { to } \\
5442 \cdot 2)\end{array}$ & $\begin{array}{r}34.6^{*}(31.0 \text { to } \\
38.8)\end{array}$ & $5.9^{*}(3.1$ to $9 \cdot 3)$ \\
\hline Sickle cell disorders & $\begin{array}{r}3250 \cdot 4(3091 \cdot 7 \\
\text { to } 3400 \cdot 5)\end{array}$ & $\begin{array}{r}77 \cdot 6^{*}(69 \cdot 9 \text { to } \\
86 \cdot 1)\end{array}$ & $\begin{array}{r}52.3^{*}(45 \cdot 8 \text { to } \\
59 \cdot 6)\end{array}$ & $\begin{array}{r}342 \cdot 1(246 \cdot 2 \\
\text { to } 455 \cdot 9)\end{array}$ & $\begin{array}{r}79.4 *(68.1 \text { to } \\
90.9)\end{array}$ & $49.7^{*}(40 \cdot 1$ to $59 \cdot 0)$ \\
\hline Sickle cell trait & $\begin{array}{r}261740 \cdot 7(249 \\
515 \cdot 4 \text { to } 272 \\
735.9)\end{array}$ & $\begin{array}{r}57.0 *(54.2 \text { to } \\
59.7)\end{array}$ & $\begin{array}{r}19 \cdot 3^{*}(17 \cdot 1 \text { to } \\
21 \cdot 3)\end{array}$ & $\begin{array}{r}1396 \cdot 6 \\
(929 \cdot 4 \text { to } \\
2004.9)\end{array}$ & $\begin{array}{r}39.0 *(32.0 \text { to } \\
49.0)\end{array}$ & $13.8^{*}(8.0$ to 23.6$)$ \\
\hline
\end{tabular}

Lancet. Author manuscript; available in PMC 2015 September 07. 


\begin{tabular}{|c|c|c|c|c|c|c|}
\hline & $\begin{array}{l}\text { Prevalent cases } \\
\text { in } 2013(x \\
1000)\end{array}$ & $\begin{array}{l}\text { Percentage } \\
\text { change in } \\
\text { prevalence } \\
\text { from } 1990 \text { to } \\
2013\end{array}$ & $\begin{array}{l}\text { Percentage } \\
\text { change in age- } \\
\text { standardised } \\
\text { prevalence from } \\
1990 \text { to } 2013\end{array}$ & $\begin{array}{l}\text { YLDs in } \\
2013(x \\
1000)\end{array}$ & $\begin{array}{l}\text { Percentage } \\
\text { change in YLDs } \\
\text { from } 1990 \text { to } \\
2013\end{array}$ & $\begin{array}{l}\text { Percentage change } \\
\text { in age- } \\
\text { standardised } \\
\text { YLDs from } 1990 \\
\text { to } 2013\end{array}$ \\
\hline Glucose-6-phosphate dehydrogen & $\begin{array}{r}\text { deripte } 6028.8(329 \\
360 \cdot 6 \text { to } 345 \\
753 \cdot 7)\end{array}$ & $\begin{array}{r}47 \cdot 1^{*}(42 \cdot 1 \text { to } \\
51 \cdot 9)\end{array}$ & $11 \cdot 4^{*}(7 \cdot 6$ to $15 \cdot 1)$ & $\begin{array}{r}36 \cdot 3(24 \cdot 8 \text { to } \\
50 \cdot 4)\end{array}$ & $\begin{array}{r}40.5 *(31.7 \text { to } \\
49.5)\end{array}$ & $11.4^{*}(4.8$ to 18.1$)$ \\
\hline $\begin{array}{c}\text { Glucose-6-phosphate } \\
\text { dehydrogenase deficiency trait }\end{array}$ & $\begin{array}{r}1181972 \cdot 5(1 \\
174352 \cdot 1 \text { to } 1 \\
188844 \cdot 0)\end{array}$ & $\begin{array}{r}38.6^{*}(37.6 \text { to } \\
39.9)\end{array}$ & $3 \cdot 0 *(2 \cdot 2$ to $3 \cdot 9)$ & $\begin{array}{r}48 \cdot 8(30 \cdot 1 \text { to } \\
73 \cdot 3)\end{array}$ & $\begin{array}{r}23.6(-1.0 \text { to } \\
50.9)\end{array}$ & $\begin{array}{r}-0.7(-20 \cdot 6 \text { to } \\
20 \cdot 4)\end{array}$ \\
\hline $\begin{array}{l}\text { Other haemoglobinopathies and } \\
\text { haemolytic anaemias }\end{array}$ & $\begin{array}{r}56010 \cdot 1(54 \\
470 \cdot 2 \text { to } 57 \\
258 \cdot 6)\end{array}$ & $\begin{array}{r}-2 \cdot 3(-5 \cdot 5 \text { to } \\
1 \cdot 3)\end{array}$ & $\begin{array}{r}-26 \cdot 6^{*}(-29 \cdot 0 \text { to } \\
-24 \cdot 0)\end{array}$ & $\begin{array}{r}1416 \cdot 5 \\
(936 \cdot 5 \text { to } \\
2051 \cdot 7)\end{array}$ & $\begin{array}{r}-12.7^{*}(-17.5 \text { to } \\
-6 \cdot 4)\end{array}$ & $-31 \cdot 4^{*}(-35 \cdot 1$ to \\
\hline $\begin{array}{l}\text { Endocrine, metabolic, blood, and } \\
\text { immune disorders }\end{array}$ & $\begin{array}{r}79556 \cdot 0(77 \\
363 \cdot 2 \text { to } 81 \\
282 \cdot 3)\end{array}$ & $\begin{array}{r}10.3^{*}(6 \cdot 9 \text { to } \\
13 \cdot 8)\end{array}$ & $\begin{array}{r}-16 \cdot 2^{*}(-18 \cdot 8 \text { to } \\
-13 \cdot 7)\end{array}$ & $\begin{array}{r}2610 \cdot 6 \\
(1797 \cdot 9 \text { to } \\
3629 \cdot 0)\end{array}$ & $6 \cdot 9^{*}(2 \cdot 2$ to $12 \cdot 8)$ & $\begin{array}{r}-16 \cdot 7^{*}(-20 \cdot 2 \text { to } \\
-12 \cdot 3)\end{array}$ \\
\hline Musculoskeletal disorders & .. &.. & .. & $\begin{array}{r}146231 \cdot 3 \\
(103763 \cdot 1 \text { to } \\
194304 \cdot 6)\end{array}$ & $\begin{array}{r}60 \cdot 7^{*}(58 \cdot 6 \text { to } \\
63 \cdot 6)\end{array}$ & $0 \cdot 6(-0 \cdot 5$ to $2 \cdot 3)$ \\
\hline Rheumatoid arthritis & $\begin{array}{r}16863 \cdot 2(16 \\
714 \cdot 4 \text { to } 17 \\
035 \cdot 8)\end{array}$ & $\begin{array}{r}57 \cdot 0 *(54 \cdot 7 \text { to } \\
59 \cdot 1)\end{array}$ & $\begin{array}{r}-5 \cdot 1 *(-6 \cdot 5 \text { to } \\
-3 \cdot 8)\end{array}$ & $\begin{array}{r}3925 \cdot 6 \\
(2818 \cdot 6 \text { to } \\
5178 \cdot 5)\end{array}$ & $\begin{array}{r}56 \cdot 8 *(53.9 \text { to } \\
59 \cdot 4)\end{array}$ & $\begin{array}{r}-4 \cdot 6^{*}(-6 \cdot 3 \text { to } \\
-3 \cdot 1)\end{array}$ \\
\hline Osteoarthritis & $\begin{array}{r}241825 \cdot 0(239 \\
655 \cdot 8 \text { to } 243 \\
896 \cdot 7)\end{array}$ & $\begin{array}{r}71.9 *(69.8 \text { to } \\
74 \cdot 3)\end{array}$ & $\begin{array}{r}-2 \cdot 6 *(-3.8 \text { to } \\
-1 \cdot 2)\end{array}$ & $\begin{array}{r}12811 \cdot 1 \\
(9030 \cdot 0 \text { to } 17 \\
281 \cdot 2)\end{array}$ & $\begin{array}{r}75.4 *(72.9 \text { to } \\
77.8)\end{array}$ & $-0 \cdot 2(-1 \cdot 6$ to $1 \cdot 2)$ \\
\hline Low back and neck pain & .. &.. & .. & $\begin{array}{r}106665 \cdot 5 \\
(74116 \cdot 9 \text { to } \\
142959 \cdot 7)\end{array}$ & $\begin{array}{r}55 \cdot 8^{*}(53.3 \text { to } \\
59 \cdot 6)\end{array}$ & $-0.4(-1.9$ to 1.9$)$ \\
\hline Low back pain & $\begin{array}{r}651008 \cdot 8(641 \\
143 \cdot 4 \text { to } 662 \\
885 \cdot 1)\end{array}$ & $\begin{array}{r}56 \cdot 8^{*}(53.6 \text { to } \\
61.4)\end{array}$ & $-0.4(-2 \cdot 4$ to $2 \cdot 5)$ & $\begin{array}{r}72317.6(49 \\
051.0 \text { to } 99 \\
738.5)\end{array}$ & $\begin{array}{r}56.7^{*}(53.5 \text { to } \\
61.5)\end{array}$ & $0 \cdot 0(-2 \cdot 0$ to $2 \cdot 9)$ \\
\hline Neck pain & $\begin{array}{r}349305 \cdot 2(341 \\
160 \cdot 3 \text { to } 359 \\
767 \cdot 4)\end{array}$ & $\begin{array}{r}54.1 *(49.1 \text { to } \\
59.9)\end{array}$ & $-1.5(-4.6$ to $2 \cdot 2)$ & $\begin{array}{r}34347.9(23 \\
792 \cdot 0 \text { to } 47 \\
418 \cdot 5)\end{array}$ & $\begin{array}{r}54.0 *(49.0 \text { to } \\
59.9)\end{array}$ & $-1 \cdot 2(-4 \cdot 3$ to $2 \cdot 6)$ \\
\hline Gout & $\begin{array}{r}5825 \cdot 6(5750 \cdot 0 \\
\text { to } 5904 \cdot 8)\end{array}$ & $\begin{array}{r}67.5^{*}(64.6 \text { to } \\
70.8)\end{array}$ & $\begin{array}{r}-2 \cdot 3^{*}(-4 \cdot 0 \text { to } \\
-0 \cdot 3)\end{array}$ & $\begin{array}{r}185.5(129 \cdot 0 \\
\text { to } 249 \cdot 2)\end{array}$ & $\begin{array}{r}67.7^{*}(62.6 \text { to } \\
73.3)\end{array}$ & $-1 \cdot 8(-4 \cdot 7$ to $1 \cdot 4)$ \\
\hline Other musculoskeletal disorders & $\begin{array}{r}248188 \cdot 2(216 \\
691 \cdot 1 \text { to } 281 \\
933 \cdot 6)\end{array}$ & $\begin{array}{r}78.4^{*}(75.0 \text { to } \\
82.5)\end{array}$ & $6 \cdot 6^{*}(5 \cdot 0$ to 8.9$)$ & $\begin{array}{r}22643 \cdot 6(15 \\
253 \cdot 8 \text { to } 31 \\
684 \cdot 6)\end{array}$ & $\begin{array}{r}78.6 * \text { (75.0 to } \\
82.9)\end{array}$ & $7 \cdot 0 *(5 \cdot 3$ to $9 \cdot 3)$ \\
\hline Other non-communicable diseases & .. & .. & .. & $\begin{array}{r}117951 \cdot 0 \\
(78473 \cdot 3 \text { to } \\
170905 \cdot 6)\end{array}$ & $\begin{array}{r}44 \cdot 8^{*}(42 \cdot 8 \text { to } \\
46 \cdot 7)\end{array}$ & $\begin{array}{r}-4 \cdot 1 *(-5 \cdot 4 \text { to } \\
-3 \cdot 0)\end{array}$ \\
\hline Congenital anomalies & .. & .. & .. & $\begin{array}{r}8022.5 \\
(5922.6 \text { to } 10 \\
168.4)\end{array}$ & $\begin{array}{r}61.4 *(52.5 \text { to } \\
72.8)\end{array}$ & $19.7^{*}$ (13.2 to 28.2$)$ \\
\hline Neural tube defects & $\begin{array}{r}1470 \cdot 2(1434 \cdot 6 \\
\text { to } 1508 \cdot 2)\end{array}$ & $\begin{array}{r}59.2^{*}(53.8 \text { to } \\
64.4)\end{array}$ & $\begin{array}{r}25 \cdot 4^{*}(21.2 \text { to } \\
29 \cdot 6)\end{array}$ & $\begin{array}{r}461 \cdot 2(325 \cdot 4 \\
\text { to } 599 \cdot 6)\end{array}$ & $\begin{array}{r}61.6^{*}(53.7 \text { to } \\
70 \cdot 7)\end{array}$ & $27.9^{*}(21.4$ to 35.1$)$ \\
\hline Congenital heart anomalies & $\begin{array}{r}34315 \cdot 9(33 \\
178.0 \text { to } 35 \\
691 \cdot 2)\end{array}$ & $\begin{array}{r}88.0 *(80.3 \text { to } \\
98.5)\end{array}$ & $\begin{array}{r}40.0 *(34.5 \text { to } \\
47.8)\end{array}$ & $\begin{array}{r}1230 \cdot 4 \\
(540 \cdot 8 \text { to } \\
2079 \cdot 5)\end{array}$ & $\begin{array}{r}86.8 *(77.3 \text { to } \\
97.6)\end{array}$ & $40.7^{*}(34.5$ to 48.5$)$ \\
\hline Orofacial clefts & $\begin{array}{r}5807.6(5621.9 \\
\text { to } 5988 \cdot 1)\end{array}$ & $\begin{array}{r}94.4^{*}(86 \cdot 1 \text { to } \\
105 \cdot 9)\end{array}$ & $\begin{array}{r}47.9^{*}(41.2 \text { to } \\
56 \cdot 6)\end{array}$ & $\begin{array}{r}68.8(45.6 \text { to } \\
98.8)\end{array}$ & $\begin{array}{r}76.3 *(65 \cdot 2 \text { to } \\
89 \cdot 5)\end{array}$ & $37 \cdot 1^{*}(28.4$ to $47 \cdot 3)$ \\
\hline Down's syndrome & $\begin{array}{r}8538 \cdot 5(8185 \cdot 7 \\
\text { to } 8897 \cdot 8)\end{array}$ & $\begin{array}{r}79.5^{*}(70 \cdot 1 \text { to } \\
88 \cdot 2)\end{array}$ & $\begin{array}{r}32.8^{*}(26.0 \text { to } \\
39 \cdot 2)\end{array}$ & $\begin{array}{r}1167 \cdot 1 \\
(894.8 \text { to } \\
1459 \cdot 7)\end{array}$ & $\begin{array}{r}91.3 *(80.5 \text { to } \\
101.4)\end{array}$ & $36.4^{*}(29.3$ to 42.9$)$ \\
\hline Turner's syndrome & $\begin{array}{r}257.5(246.6 \text { to } \\
271 \cdot 2)\end{array}$ & $\begin{array}{r}51.2 *(42.6 \text { to } \\
62.3)\end{array}$ & $14.8 *$ ( $8 \cdot 4$ to $23 \cdot 3)$ & $\begin{array}{r}4.3(2 \cdot 2 \text { to } \\
6 \cdot 9)\end{array}$ & $\begin{array}{r}52.1 *(42.9 \text { to } \\
64.8)\end{array}$ & $14.5^{*}(7.5$ to 23.8$)$ \\
\hline Klinefelter's syndrome & $\begin{array}{r}216 \cdot 3(207 \cdot 1 \text { to } \\
226 \cdot 7)\end{array}$ & $\begin{array}{r}42.0 *(33.2 \text { to } \\
52.7)\end{array}$ & $6.5^{*}(0.0$ to 14.5$)$ & $\begin{array}{r}1.3(0 \cdot 6 \text { to } \\
2 \cdot 4)\end{array}$ & $\begin{array}{r}47.2 *(37.0 \text { to } \\
58.8)\end{array}$ & $5 \cdot 5(-1.8$ to 13.9$)$ \\
\hline \multicolumn{2}{|c|}{$\begin{array}{r}\text { Chromosomal unbalanced rearrangements } 11649 \cdot 3(11 \\
154 \cdot 1 \text { to } 12 \\
235 \cdot 1)\end{array}$} & $\begin{array}{r}74.0^{*}(66 \cdot 0 \text { to } \\
83 \cdot 0)\end{array}$ & $\begin{array}{r}27.5^{*}(21.7 \text { to } \\
34.0)\end{array}$ & $\begin{array}{r}1602 \cdot 9 \\
(1203 \cdot 6 \text { to } \\
2038 \cdot 4)\end{array}$ & $\begin{array}{r}85 \cdot 1 *(75 \cdot 6 \text { to } \\
95 \cdot 0)\end{array}$ & $30 \cdot 6^{*}(24.8$ to $37 \cdot 1)$ \\
\hline
\end{tabular}

Lancet. Author manuscript; available in PMC 2015 September 07. 


\begin{tabular}{|c|c|c|c|c|c|c|}
\hline & $\begin{array}{l}\text { Prevalent cases } \\
\text { in 2013 }(\times \\
1000)\end{array}$ & $\begin{array}{l}\text { Percentage } \\
\text { change in } \\
\text { prevalence } \\
\text { from } 1990 \text { to } \\
2013\end{array}$ & $\begin{array}{l}\text { Percentage } \\
\text { change in age- } \\
\text { standardised } \\
\text { prevalence from } \\
1990 \text { to } 2013\end{array}$ & $\begin{array}{l}\text { YLDs in } \\
2013(x \\
1000)\end{array}$ & $\begin{array}{l}\text { Percentage } \\
\text { change in YLDs } \\
\text { from } 1990 \text { to } \\
2013\end{array}$ & $\begin{array}{l}\text { Percentage change } \\
\text { in age- } \\
\text { standardised } \\
\text { YLDs from } 1990 \\
\text { to } 2013\end{array}$ \\
\hline Other congenital anomalies & $\begin{array}{r}25840 \cdot 0(22 \\
170 \cdot 5 \text { to } 29 \\
283 \cdot 1)\end{array}$ & $\begin{array}{r}22.9 *(17.2 \text { to } \\
28 \cdot 7)\end{array}$ & $\begin{array}{r}-10 \cdot 6^{*}(-14 \cdot 7 \text { to } \\
-6 \cdot 5)\end{array}$ & $\begin{array}{r}3486 \cdot 4 \\
(2436 \cdot 6 \text { to } \\
4641 \cdot 2)\end{array}$ & $\begin{array}{r}38.7 *(26.5 \text { to } \\
56 \cdot 4)\end{array}$ & $4 \cdot 3(-4 \cdot 6$ to $17 \cdot 4)$ \\
\hline Skin and subcutaneous diseases & .. & .. & .. & $\begin{array}{r}39051 \cdot 0(25 \\
044.5 \text { to } 60 \\
125 \cdot 8)\end{array}$ & $\begin{array}{r}37.3^{*}(33.8 \text { to } \\
40 \cdot 3)\end{array}$ & $0 \cdot 3(-2 \cdot 0$ to $2 \cdot 3)$ \\
\hline Dermatitis & $\begin{array}{r}333785 \cdot 4(289 \\
927 \cdot 3 \text { to } 381 \\
312 \cdot 4)\end{array}$ & $\begin{array}{r}38.7^{*}(36 \cdot 2 \text { to } \\
41 \cdot 1)\end{array}$ & $\begin{array}{r}-1.7^{*}(-2.4 \text { to } \\
-0.9)\end{array}$ & $\begin{array}{r}9278.4 \\
(6029.0 \text { to } 13 \\
326 \cdot 7)\end{array}$ & $\begin{array}{r}36.8 *(34.7 \text { to } \\
39.2)\end{array}$ & $-0 \cdot 6(-1 \cdot 4$ to $0 \cdot 3)$ \\
\hline Psoriasis & $\begin{array}{r}58264 \cdot 1(51 \\
720 \cdot 2 \text { to } 64 \\
609 \cdot 4)\end{array}$ & $\begin{array}{r}46.0 * \\
(44.0 \text { to } \\
48.8)\end{array}$ & $\begin{array}{r}-2.2^{*}(-3.0 \text { to } \\
-1.6)\end{array}$ & $\begin{array}{r}4726 \cdot 7 \\
(3254.7 \text { to } \\
6621.9)\end{array}$ & $\begin{array}{r}45.9 *(43.2 \text { to } \\
48.8)\end{array}$ & $\begin{array}{r}-1.9^{*}(-3.0 \text { to } \\
-0.6)\end{array}$ \\
\hline Cellulitis & $\begin{array}{r}1705 \cdot 9(1429 \cdot 7 \\
\text { to } 2040 \cdot 3)\end{array}$ & $\begin{array}{r}19 \cdot 1^{*}(13 \cdot 2 \text { to } \\
25 \cdot 1)\end{array}$ & $\begin{array}{r}-18.2^{*}(-21.2 \text { to } \\
-15.9)\end{array}$ & $\begin{array}{r}120 \cdot 2(78 \cdot 6 \\
\text { to } 170 \cdot 2)\end{array}$ & $\begin{array}{r}18 \cdot 6 *(11 \cdot 3 \text { to } \\
26 \cdot 6)\end{array}$ & $\begin{array}{r}-18.0 *(-21.9 \text { to } \\
-13 \cdot 8)\end{array}$ \\
\hline Bacterial skin diseases & $\begin{array}{r}5759 \cdot 7(4693.5 \\
\text { to } 7278.2)\end{array}$ & $\begin{array}{r}-6 \cdot 7(-16 \cdot 2 \text { to } \\
3 \cdot 7)\end{array}$ & $\begin{array}{r}-23.8 *(-29.9 \text { to } \\
-17.6)\end{array}$ & $\begin{array}{r}32 \cdot 6(12 \cdot 4 \text { to } \\
72 \cdot 1)\end{array}$ & $\begin{array}{r}-7 \cdot 1(-16 \cdot 3 \text { to } \\
2 \cdot 9)\end{array}$ & $\begin{array}{r}-24.0 *(-29 \cdot 8 \text { to } \\
-17 \cdot 7)\end{array}$ \\
\hline Scabies & $\begin{array}{r}66107.5(62 \\
430 \cdot 1 \text { to } 70 \\
635 \cdot 9)\end{array}$ & $\begin{array}{r}24.8 *(15 \cdot 0 \text { to } \\
40 \cdot 1)\end{array}$ & $-4 \cdot 0(-11 \cdot 3$ to $7 \cdot 3)$ & $\begin{array}{r}1705.4 \\
(967.2 \text { to } \\
2711.6)\end{array}$ & $\begin{array}{r}24.8 * \text { (14.8 to } \\
40 \cdot 3)\end{array}$ & $-3.7(-11.2$ to 7.8$)$ \\
\hline Fungal skin diseases & $\begin{array}{r}683713 \cdot 8(597 \\
898 \cdot 2 \text { to } 780 \\
963.4)\end{array}$ & $\begin{array}{r}44.2^{*}(41.2 \text { to } \\
47.9)\end{array}$ & $3.0 *(1.9$ to 3.8$)$ & $\begin{array}{r}3847 \cdot 2 \\
(1574 \cdot 5 \text { to } \\
8139 \cdot 8)\end{array}$ & $\begin{array}{r}44.1 *(41.1 \text { to } \\
47 \cdot 9)\end{array}$ & $3 \cdot 3(2 \cdot 2$ to $4 \cdot 1)$ \\
\hline Viral skin diseases & $\begin{array}{r}127923.6(105 \\
593 \cdot 4 \text { to } 151 \\
635 \cdot 9)\end{array}$ & $\begin{array}{r}20.5^{*}(17.9 \text { to } \\
24.3)\end{array}$ & $\begin{array}{r}-1 \cdot 8^{*}(-3 \cdot 1 \text { to } \\
-0 \cdot 3)\end{array}$ & $\begin{array}{r}3955 \cdot 0 \\
(2398.4 \text { to } \\
6150.9)\end{array}$ & $\begin{array}{r}20 \cdot 4^{*}(17 \cdot 8 \text { to } \\
24 \cdot 1)\end{array}$ & $-1 \cdot 7(-3 \cdot 0$ to $0 \cdot 0)$ \\
\hline Acne vulgaris & $\begin{array}{r}661634.9(622 \\
842 \cdot 5 \text { to } 700 \\
241.7)\end{array}$ & $\begin{array}{r}23.2^{*}(13.9 \text { to } \\
32 \cdot 6)\end{array}$ & $-0.4(-7.8$ to 7.3$)$ & $\begin{array}{r}7180 \cdot 8 \\
(3451 \cdot 6 \text { to } 13 \\
214 \cdot 1)\end{array}$ & $\begin{array}{r}23 \cdot 2 *(13 \cdot 7 \text { to } \\
32 \cdot 7)\end{array}$ & $-0 \cdot 3(-7 \cdot 7$ to $7 \cdot 4)$ \\
\hline Alopecia areata & $\begin{array}{r}8775 \cdot 5(8614.4 \\
\text { to } 8937.9)\end{array}$ & $\begin{array}{r}46 \cdot 1 *(42.5 \text { to } \\
49.4)\end{array}$ & $-1 \cdot 1(-3 \cdot 4$ to $1 \cdot 2)$ & $\begin{array}{r}292 \cdot 4(186 \cdot 8 \\
\text { to } 435 \cdot 2)\end{array}$ & $\begin{array}{r}45 \cdot 6 *(41 \cdot 2 \text { to } \\
50 \cdot 0)\end{array}$ & $-0 \cdot 9(-3.6$ to 1.8$)$ \\
\hline Pruritus & $\begin{array}{r}1024 \cdot 6(988 \cdot 1 \\
\text { to } 1074 \cdot 1)\end{array}$ & $\begin{array}{r}55 \cdot 3^{*}(46 \cdot 3 \text { to } \\
63 \cdot 1)\end{array}$ & $-0 \cdot 2(-5 \cdot 9$ to $5 \cdot 3)$ & $\begin{array}{r}10 \cdot 8(5 \cdot 1 \text { to } \\
20 \cdot 0)\end{array}$ & $\begin{array}{r}54.8 * \\
(44.9 \text { to } \\
64.9)\end{array}$ & $0.0(-6 \cdot 4$ to $6 \cdot 5)$ \\
\hline Urticaria & $\begin{array}{r}79582 \cdot 8(72 \\
812 \cdot 3 \text { to } 86 \\
296 \cdot 3)\end{array}$ & $\begin{array}{r}60.5 *(44.2 \text { to } \\
75 \cdot 0)\end{array}$ & $7 \cdot 3(-3.8$ to $16 \cdot 6)$ & $\begin{array}{r}4720 \cdot 7 \\
(3036.5 \text { to } \\
6737.2)\end{array}$ & $\begin{array}{r}60 \cdot 7^{*}(43.4 \text { to } \\
75 \cdot 5)\end{array}$ & $7.7(-3.5$ to 17.6$)$ \\
\hline Decubitus ulcer & $\begin{array}{r}1921 \cdot 4(1853 \cdot 0 \\
\text { to } 2001 \cdot 2)\end{array}$ & $\begin{array}{r}59.5^{*}(51.8 \text { to } \\
67.1)\end{array}$ & $\begin{array}{r}-8 \cdot 1^{*}(-13 \cdot 1 \text { to } \\
-3 \cdot 3)\end{array}$ & $\begin{array}{r}277.5(196 \cdot 0 \\
\text { to } 371 \cdot 2)\end{array}$ & $\begin{array}{r}58.1 * \text { (49.8 to } \\
66.8)\end{array}$ & $\begin{array}{r}-7.7^{*}(-12.9 \text { to } \\
-2.5)\end{array}$ \\
\hline $\begin{array}{l}\text { Other skin and subcutaneous } \\
\text { diseases }\end{array}$ & $\begin{array}{r}495327 \cdot 6(319 \\
462 \cdot 3 \text { to } 761 \\
255 \cdot 5)\end{array}$ & $\begin{array}{r}57.1 *(48.5 \text { to } \\
64.4)\end{array}$ & $0.0(-0.9$ to 0.9$)$ & $\begin{array}{r}2903 \cdot 1 \\
(1274 \cdot 1 \text { to } \\
6123 \cdot 6)\end{array}$ & $\begin{array}{r}57.0 *(48.5 \text { to } \\
64.5)\end{array}$ & $0.4(-0.6$ to 1.3$)$ \\
\hline Sense organ diseases & .. & .. & .. & $\begin{array}{r}54428 \cdot 1(36 \\
458.4 \text { to } 76 \\
075.4)\end{array}$ & $\begin{array}{r}47 \cdot 4^{*}(44 \cdot 1 \text { to } \\
50 \cdot 1)\end{array}$ & $\begin{array}{r}-8.2^{*}(-9.7 \text { to } \\
-6.7)\end{array}$ \\
\hline Glaucoma & $\begin{array}{r}10899.9(9853 \\
2 \text { to } 12002 \cdot 8)\end{array}$ & $\begin{array}{r}63.0^{*}(50.2 \text { to } \\
74.7)\end{array}$ & $-3 \cdot 5(-10 \cdot 6$ to $2 \cdot 9)$ & $\begin{array}{r}807.5(571.6 \\
\text { to } 1102 \cdot 8)\end{array}$ & $\begin{array}{r}63 \cdot 1 *(54.8 \text { to } \\
70 \cdot 9)\end{array}$ & $\begin{array}{r}-4.6^{*}(-9.7 \text { to } \\
-0.2)\end{array}$ \\
\hline Cataract & $\begin{array}{r}44221 \cdot 6(39 \\
326 \cdot 0 \text { to } 48 \\
603 \cdot 1)\end{array}$ & $\begin{array}{r}47.5^{*}(41.3 \text { to } \\
56 \cdot 4)\end{array}$ & $\begin{array}{r}-17 \cdot 6^{*}(-20 \cdot 8 \text { to } \\
-13 \cdot 0)\end{array}$ & $\begin{array}{r}2916 \cdot 7 \\
(2055 \cdot 1 \text { to } \\
3962 \cdot 2)\end{array}$ & $\begin{array}{r}56.7 *(50 \cdot 6 \text { to } \\
63.9)\end{array}$ & $\begin{array}{r}-12.5^{*}(-15 \cdot 7 \text { to } \\
-8 \cdot 6)\end{array}$ \\
\hline Macular degeneration & $\begin{array}{r}13873 \cdot 8(12 \\
176 \cdot 5 \text { to } 15 \\
562 \cdot 6)\end{array}$ & $\begin{array}{r}76 \cdot 3^{*}(69 \cdot 4 \text { to } \\
89 \cdot 3)\end{array}$ & $-1 \cdot 6(-5 \cdot 6$ to $5 \cdot 1)$ & $\begin{array}{r}725 \cdot 6(509 \cdot 4 \\
\text { to } 985 \cdot 1)\end{array}$ & $\begin{array}{r}75.0 *(69.0 \text { to } \\
87.8)\end{array}$ & $-4.4(-8 \cdot 6$ to 1.4$)$ \\
\hline Uncorrected refractive error & $\begin{array}{r}659847 \cdot 2(648 \\
298 \cdot 7 \text { to } 671 \\
446 \cdot 7)\end{array}$ & $\begin{array}{r}52.0 *(49.0 \text { to } \\
55 \cdot 0)\end{array}$ & $\begin{array}{r}-5 \cdot 2^{*}(-7 \cdot 0 \text { to } \\
-3 \cdot 4)\end{array}$ & $\begin{array}{r}11257 \cdot 2 \\
(7149 \cdot 8 \text { to } 17 \\
452 \cdot 3)\end{array}$ & $\begin{array}{r}43 \cdot 5^{*}(40 \cdot 4 \text { to } \\
47 \cdot 2)\end{array}$ & $\begin{array}{r}-8 \cdot 9 *(-10 \cdot 6 \text { to } \\
-7 \cdot 0)\end{array}$ \\
\hline loss $\quad$ Age-related and other hearing & $\begin{array}{r}1128939 \cdot 1(1 \\
055657 \cdot 5 \text { to } 1 \\
200476 \cdot 4)\end{array}$ & $\begin{array}{r}55 \cdot 7^{*}(52.7 \text { to } \\
58.4)\end{array}$ & $\begin{array}{r}-6 \cdot 5^{*}(-7 \cdot 9 \text { to } \\
-5 \cdot 2)\end{array}$ & $\begin{array}{r}32579 \cdot 7(22 \\
083 \cdot 7 \text { to } 45 \\
846 \cdot 1)\end{array}$ & $\begin{array}{r}50.7^{*}(45.4 \text { to } \\
54.8)\end{array}$ & $\begin{array}{r}-7.9^{*}(-10 \cdot 2 \text { to } \\
-5.4)\end{array}$ \\
\hline Other vision loss & $\begin{array}{r}26806 \cdot 0(24 \\
116 \cdot 0 \text { to } 29 \\
696 \cdot 9)\end{array}$ & $\begin{array}{r}19.9^{*}(16.0 \text { to } \\
23 \cdot 8)\end{array}$ & $\begin{array}{r}-22 \cdot 2^{*}(-25.0 \text { to } \\
-19.9)\end{array}$ & $\begin{array}{r}1793 \cdot 5 \\
(1260 \cdot 4 \text { to } \\
2452 \cdot 0)\end{array}$ & $\begin{array}{r}23.7^{*}(19.4 \text { to } \\
27.6)\end{array}$ & $\begin{array}{r}-21 \cdot 1^{*}(-23 \cdot 5 \text { to } \\
-19 \cdot 0)\end{array}$ \\
\hline
\end{tabular}

Lancet. Author manuscript; available in PMC 2015 September 07. 


\begin{tabular}{|c|c|c|c|c|c|c|}
\hline & $\begin{array}{l}\text { Prevalent cases } \\
\text { in 2013 }(\times \\
1000)\end{array}$ & $\begin{array}{l}\text { Percentage } \\
\text { change in } \\
\text { prevalence } \\
\text { from } 1990 \text { to } \\
2013\end{array}$ & $\begin{array}{l}\text { Percentage } \\
\text { change in age- } \\
\text { standardised } \\
\text { prevalence from } \\
1990 \text { to } 2013\end{array}$ & $\begin{array}{l}\text { YLDs in } \\
2013(x \\
1000)\end{array}$ & $\begin{array}{l}\text { Percentage } \\
\text { change in YLDs } \\
\text { from } 1990 \text { to } \\
2013\end{array}$ & $\begin{array}{l}\text { Percentage change } \\
\text { in age- } \\
\text { standardised } \\
\text { YLDs from } 1990 \\
\text { to } 2013\end{array}$ \\
\hline Other sense organ diseases & $\begin{array}{r}164612.2(163 \\
116.9 \text { to } 166 \\
389.9)\end{array}$ & $\begin{array}{r}34.2^{*}(32.5 \text { to } \\
36 \cdot 2)\end{array}$ & $-0 \cdot 2(-1.4$ to 1.2$)$ & $\begin{array}{r}4348 \cdot 0 \\
(2704 \cdot 3 \text { to } \\
6435 \cdot 1)\end{array}$ & $\begin{array}{r}33.7 *(31.7 \text { to } \\
35.8)\end{array}$ & $0 \cdot 1(-1 \cdot 3$ to $1 \cdot 6)$ \\
\hline Oral disorders &.. &.. &.. & $\begin{array}{r}16449 \cdot 5(10 \\
022 \cdot 3 \text { to } 25 \\
506 \cdot 3)\end{array}$ & $\begin{array}{r}47.6 *(45.8 \text { to } \\
49.7)\end{array}$ & $\begin{array}{r}-7.7^{*}(-9.7 \text { to } \\
-5.5)\end{array}$ \\
\hline Deciduous caries & $\begin{array}{r}492920 \cdot 5(490 \\
657 \cdot 1 \text { to } 495 \\
385 \cdot 2)\end{array}$ & $4 \cdot 4^{*}(3.6$ to $5 \cdot 2)$ & $\begin{array}{r}-2 \cdot 1 *(-2 \cdot 8 \text { to } \\
-1 \cdot 4)\end{array}$ & $\begin{array}{r}181 \cdot 1(79 \cdot 0 \\
\text { to } 350 \cdot 9)\end{array}$ & $5 \cdot 7^{*}(4 \cdot 1$ to $7 \cdot 5)$ & $-0 \cdot 9(-2 \cdot 3$ to $0 \cdot 8)$ \\
\hline Permanent caries & $\begin{array}{r}2633328 \cdot 8(2 \\
607931.9 \text { to } 2 \\
658749 \cdot 5)\end{array}$ & $\begin{array}{r}37 \cdot 4^{*}(35 \cdot 8 \text { to } \\
39 \cdot 6)\end{array}$ & $-1 \cdot 2(-2 \cdot 3$ to $0 \cdot 4)$ & $\begin{array}{r}2411 \cdot 0 \\
(1102 \cdot 6 \text { to } \\
4664 \cdot 5)\end{array}$ & $\begin{array}{r}40 \cdot 5^{*}(38 \cdot 3 \text { to } \\
43 \cdot 0)\end{array}$ & $1.8^{*}(0.1$ to 3.7$)$ \\
\hline Periodontal diseases & $\begin{array}{r}503967 \cdot 2(496 \\
870 \cdot 4 \text { to } 511 \\
448 \cdot 4)\end{array}$ & $\begin{array}{r}66.7^{*}(63.7 \text { to } \\
70 \cdot 1)\end{array}$ & $1.4(-0.4$ to $3 \cdot 4)$ & $\begin{array}{r}3286 \cdot 0 \\
(1318 \cdot 3 \text { to } \\
6750 \cdot 3)\end{array}$ & $\begin{array}{r}66 \cdot 8^{*}(63 \cdot 8 \text { to } \\
70 \cdot 3)\end{array}$ & $1.7(-0.2$ to $3 \cdot 7)$ \\
\hline Edentulism and severe tooth loss & $\begin{array}{r}250683 \cdot 6(247 \\
512 \cdot 3 \text { to } 253 \\
463 \cdot 0)\end{array}$ & $\begin{array}{r}45.6^{*}(43.4 \text { to } \\
47.9)\end{array}$ & $\begin{array}{r}-16 \cdot 4^{*}(-17 \cdot 7 \text { to } \\
-15 \cdot 1)\end{array}$ & $\begin{array}{r}6855 \cdot 6 \\
(4647 \cdot 2 \text { to } \\
9420 \cdot 4)\end{array}$ & $\begin{array}{r}45 \cdot 6^{*}(43.3 \text { to } \\
47.9)\end{array}$ & $\begin{array}{r}-16 \cdot 2^{*}(-17 \cdot 6 \text { to } \\
-14 \cdot 9)\end{array}$ \\
\hline Other oral disorders & $\begin{array}{r}126945 \cdot 5(125 \\
452 \cdot 3 \text { to } 128 \\
568 \cdot 2)\end{array}$ & $\begin{array}{r}44.7^{*}(42 \cdot 5 \text { to } \\
46 \cdot 9)\end{array}$ & $-0.5(-2.0$ to 0.9$)$ & $\begin{array}{r}3715 \cdot 7 \\
(2347.5 \text { to } \\
5558.6)\end{array}$ & $\begin{array}{r}44 \cdot 6^{*}(42 \cdot 2 \text { to } \\
46 \cdot 9)\end{array}$ & $-0 \cdot 3(-1.9$ to 1.2$)$ \\
\hline Injuries & .. &.. & .. & $\begin{array}{r}36831 \cdot 5(26 \\
895 \cdot 7 \text { to } 48 \\
748 \cdot 0)\end{array}$ & $\begin{array}{r}0 \cdot 3(-13 \cdot 7 \text { to } \\
11 \cdot 3)\end{array}$ & $\begin{array}{r}-37 \cdot 0 *(-45 \cdot 4 \text { to } \\
-30 \cdot 0)\end{array}$ \\
\hline Transport injuries & .. & .. & .. & $\begin{array}{r}10194 \cdot 9 \\
(7477 \cdot 9 \text { to } 13 \\
437 \cdot 8)\end{array}$ & $6 \cdot 2(-1 \cdot 0$ to $14 \cdot 5)$ & $\begin{array}{r}-31 \cdot 7^{*}(-36 \cdot 2 \text { to } \\
-26 \cdot 8)\end{array}$ \\
\hline Road injuries & .. & .. & .. & $\begin{array}{r}8593 \cdot 4 \\
(6305 \cdot 9 \text { to } 11 \\
313 \cdot 7)\end{array}$ & $6.9(-0.7$ to $15 \cdot 8)$ & $\begin{array}{r}-31.0^{*}(-35 \cdot 8 \text { to } \\
-25 \cdot 8)\end{array}$ \\
\hline Pedestrian road injuries & $\begin{array}{r}37844 \cdot 1(37 \\
457 \cdot 1 \text { to } 38 \\
216 \cdot 7)\end{array}$ & $\begin{array}{r}53.7^{*}(51.7 \text { to } \\
55.6)\end{array}$ & $0.6(-0.7$ to 1.9$)$ & $\begin{array}{r}2271 \cdot 8 \\
(1657 \cdot 1 \text { to } \\
2993 \cdot 5)\end{array}$ & $\begin{array}{r}17.2 *(8.9 \text { to } \\
27.0)\end{array}$ & $\begin{array}{r}-23.6^{*}(-28.5 \text { to } \\
-17.8)\end{array}$ \\
\hline Cyclist road injuries & $\begin{array}{r}13678 \cdot 6(13 \\
439 \cdot 2 \text { to } 13 \\
925 \cdot 1)\end{array}$ & $\begin{array}{r}24 \cdot 1^{*}(22 \cdot 1 \text { to } \\
26 \cdot 2)\end{array}$ & $\begin{array}{r}-14.9 *(-16 \cdot 3 \text { to } \\
-13 \cdot 4)\end{array}$ & $\begin{array}{r}812 \cdot 4(594 \cdot 6 \\
\text { to } 1074 \cdot 2)\end{array}$ & $2 \cdot 8(-3.5$ to 9.9$)$ & $\begin{array}{r}-33.9^{*}(-37.9 \text { to } \\
-29.5)\end{array}$ \\
\hline Motorcyclist road injuries & $\begin{array}{r}32483.6(31 \\
964.6 \text { to } 33 \\
019 \cdot 7)\end{array}$ & $\begin{array}{r}28 \cdot 0 *(25 \cdot 5 \text { to } \\
30 \cdot 0)\end{array}$ & $\begin{array}{r}-17.2^{*}(-18.9 \text { to } \\
-15.9)\end{array}$ & $\begin{array}{r}1649 \cdot 2 \\
(1201 \cdot 7 \text { to } \\
2204.5)\end{array}$ & $\begin{array}{r}-4 \cdot 2(-12 \cdot 2 \text { to } \\
5 \cdot 1)\end{array}$ & $\begin{array}{r}-38 \cdot 2^{*}(-43 \cdot 1 \text { to } \\
-32 \cdot 5)\end{array}$ \\
\hline Motor vehicle road injuries & $\begin{array}{r}60733 \cdot 7(59 \\
848 \cdot 5 \text { to } 61 \\
593 \cdot 1)\end{array}$ & $\begin{array}{r}40 \cdot 8^{*}(39 \cdot 3 \text { to } \\
42 \cdot 4)\end{array}$ & $\begin{array}{r}-2.7^{*}(-3.7 \text { to } \\
-1.5)\end{array}$ & $\begin{array}{r}3777 \cdot 8 \\
(2811 \cdot 0 \text { to } \\
4946 \cdot 9)\end{array}$ & $8 \cdot 1^{*}(0.3$ to $16 \cdot 7)$ & $\begin{array}{r}-30 \cdot 9^{*}(-35 \cdot 6 \text { to } \\
-25 \cdot 8)\end{array}$ \\
\hline Other road injuries & $\begin{array}{r}1371.5(1351 \cdot 1 \\
\text { to } 1393.6)\end{array}$ & $\begin{array}{r}17.9 *(15.9 \text { to } \\
19.8)\end{array}$ & $\begin{array}{r}-22.8^{*}(-24.2 \text { to } \\
-21.5)\end{array}$ & $\begin{array}{r}82 \cdot 3(61.0 \text { to } \\
107 \cdot 3)\end{array}$ & $\begin{array}{r}-5 \cdot 7(-11 \cdot 4 \text { to } \\
1 \cdot 0)\end{array}$ & $\begin{array}{r}-40 \cdot 1^{*}(-43 \cdot 4 \text { to } \\
-36 \cdot 1)\end{array}$ \\
\hline Other transport injuries & $\begin{array}{r}26293 \cdot 0(25 \\
899 \cdot 6 \text { to } 26 \\
709 \cdot 0)\end{array}$ & $\begin{array}{r}22.9 *(20 \cdot 3 \text { to } \\
25 \cdot 2)\end{array}$ & $\begin{array}{r}-21.7^{*}(-23 \cdot 5 \text { to } \\
-20 \cdot 1)\end{array}$ & $\begin{array}{r}1601 \cdot 5 \\
(1173 \cdot 2 \text { to } \\
2118 \cdot 5)\end{array}$ & $2 \cdot 2(-3 \cdot 1$ to $8 \cdot 5)$ & $\begin{array}{r}-35 \cdot 1^{*}(-38 \cdot 5 \text { to } \\
-31 \cdot 2)\end{array}$ \\
\hline Unintentional injuries & .. & .. & .. & $\begin{array}{r}21649 \cdot 4(16 \\
036 \cdot 0 \text { to } 28 \\
726 \cdot 9)\end{array}$ & $\begin{array}{r}17 \cdot 9^{*}(10 \cdot 5 \text { to } \\
25 \cdot 8)\end{array}$ & $\begin{array}{r}-28 \cdot 0 *(-32 \cdot 8 \text { to } \\
-22 \cdot 7)\end{array}$ \\
\hline Falls & $\begin{array}{r}250044 \cdot 4(247 \\
438 \cdot 2 \text { to } 252 \\
548 \cdot 9)\end{array}$ & $\begin{array}{r}61.3^{*}(59.7 \text { to } \\
62 \cdot 8)\end{array}$ & $0.9(-0.2$ to $1 \cdot 8)$ & $\begin{array}{r}12818.1 \\
(9356.9 \text { to } 16 \\
996.8)\end{array}$ & $\begin{array}{r}23.5 *(13.6 \text { to } \\
35 \cdot 0)\end{array}$ & $\begin{array}{r}-28.3^{*}(-34.5 \text { to } \\
-21 \cdot 1)\end{array}$ \\
\hline Drowning & $\begin{array}{r}6149 \cdot 1(6022 \cdot 3 \\
\text { to } 6275 \cdot 4)\end{array}$ & $\begin{array}{r}13 \cdot 7^{*}(10 \cdot 6 \text { to } \\
16 \cdot 7)\end{array}$ & $\begin{array}{r}-23.9 *(-25.9 \text { to } \\
-21.9)\end{array}$ & $\begin{array}{r}373 \cdot 6(273 \cdot 4 \\
\text { to } 485 \cdot 1)\end{array}$ & $\begin{array}{r}-8 \cdot 6^{*}(-13 \cdot 5 \text { to } \\
-2 \cdot 4)\end{array}$ & $\begin{array}{r}-37.9 *(-40.9 \text { to } \\
-34.1)\end{array}$ \\
\hline Fire, heat, and hot substances & $\begin{array}{r}35326 \cdot 2(34 \\
097 \cdot 3 \text { to } 36 \\
575 \cdot 8)\end{array}$ & $\begin{array}{r}7.4^{*}(3.4 \text { to } \\
11.8)\end{array}$ & $\begin{array}{r}-28.7^{*}(-31.4 \text { to } \\
-25.5)\end{array}$ & $\begin{array}{r}1172 \cdot 2 \\
(867 \cdot 1 \text { to } \\
1551 \cdot 4)\end{array}$ & $\begin{array}{r}-4.7(-10.0 \text { to } \\
0 \cdot 6)\end{array}$ & $\begin{array}{r}-36.8 *(-39.8 \text { to } \\
-33.8)\end{array}$ \\
\hline
\end{tabular}




\begin{tabular}{|c|c|c|c|c|c|c|}
\hline & $\begin{array}{l}\text { Prevalent cases } \\
\text { in 2013 }(\times \\
1000)\end{array}$ & $\begin{array}{l}\text { Percentage } \\
\text { change in } \\
\text { prevalence } \\
\text { from } 1990 \text { to } \\
2013\end{array}$ & $\begin{array}{l}\text { Percentage } \\
\text { change in age- } \\
\text { standardised } \\
\text { prevalence from } \\
1990 \text { to } 2013\end{array}$ & $\begin{array}{l}\text { YLDs in } \\
2013(x \\
1000)\end{array}$ & $\begin{array}{l}\text { Percentage } \\
\text { change in YLDs } \\
\text { from } 1990 \text { to } \\
2013\end{array}$ & $\begin{array}{l}\text { Percentage change } \\
\text { in age- } \\
\text { standardised } \\
\text { YLDs from } 1990 \\
\text { to } 2013\end{array}$ \\
\hline Poisonings & $\begin{array}{r}925 \cdot 2(910 \cdot 3 \text { to } \\
939 \cdot 5)\end{array}$ & $5.1 *(2.8$ to 7.0$)$ & $\begin{array}{r}-29.2 *(-30 \cdot 7 \text { to } \\
-27.9)\end{array}$ & $\begin{array}{r}78 \cdot 7(56 \cdot 2 \text { to } \\
104 \cdot 5)\end{array}$ & $\begin{array}{r}-6 \cdot 3^{*}(-9 \cdot 8 \text { to } \\
-2 \cdot 7)\end{array}$ & $\begin{array}{r}-36 \cdot 7^{*}(-39 \cdot 0 \text { to } \\
-34 \cdot 4)\end{array}$ \\
\hline Exposure to mechanical forces & .. & .. &.. & $\begin{array}{r}3758 \cdot 0 \\
(2748 \cdot 6 \text { to } \\
4985 \cdot 4)\end{array}$ & $5 \cdot 2^{*}(0.3$ to $10 \cdot 4)$ & $\begin{array}{r}-30 \cdot 1^{*}(-33 \cdot 0 \text { to } \\
-26 \cdot 9)\end{array}$ \\
\hline Unintentional firearm injuries & $\begin{array}{r}3729.0(3639 \cdot 3 \\
\text { to } 3820 \cdot 9)\end{array}$ & 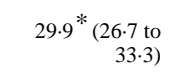 & $\begin{array}{r}-13 \cdot 3^{*}(-15 \cdot 4 \text { to } \\
-11 \cdot 1)\end{array}$ & $\begin{array}{r}146 \cdot 6(109 \cdot 0 \\
\quad \text { to } 194 \cdot 3)\end{array}$ & $6 \cdot 4(-0 \cdot 3$ to $14 \cdot 2)$ & $\begin{array}{r}-29 \cdot 8^{*}(-34 \cdot 0 \text { to } \\
-25 \cdot 1)\end{array}$ \\
\hline Unintentional suffocation & $\begin{array}{r}949 \cdot 6(923.4 \text { to } \\
979 \cdot 8)\end{array}$ & $\begin{array}{r}66.5 *(63.2 \text { to } \\
69 \cdot 4)\end{array}$ & $\begin{array}{r}13.4 *(11.6 \text { to } \\
15.0)\end{array}$ & $\begin{array}{r}54 \cdot 2(40 \cdot 2 \text { to } \\
71 \cdot 6)\end{array}$ & $\begin{array}{r}42 \cdot 2 *(34.7 \text { to } \\
50 \cdot 1)\end{array}$ & $-3 \cdot 3(-8 \cdot 1$ to $1 \cdot 6)$ \\
\hline $\begin{array}{l}\text { Other exposure to mechanical } \\
\text { forces }\end{array}$ & $\begin{array}{r}113111.5(105 \\
859.7 \text { to } 120 \\
514 \cdot 4)\end{array}$ & $\begin{array}{r}15 \cdot 7^{*}(12 \cdot 9 \text { to } \\
18 \cdot 0)\end{array}$ & 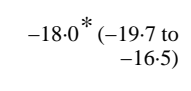 & $\begin{array}{r}3557 \cdot 2 \\
(2597 \cdot 7 \text { to } \\
4726 \cdot 4)\end{array}$ & $4.7(0.0$ to 9.8$)$ & $\begin{array}{r}-30 \cdot 4^{*}(-33 \cdot 3 \text { to } \\
-27 \cdot 3)\end{array}$ \\
\hline $\begin{array}{l}\text { Adverse effects of medical } \\
\text { treatment }\end{array}$ & $\begin{array}{r}1526 \cdot 1(1226 \cdot 7 \\
\text { to } 1820 \cdot 2)\end{array}$ & 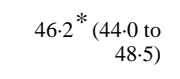 & $-1 \cdot 0(-2 \cdot 4$ to $0 \cdot 4)$ & $\begin{array}{r}201 \cdot 0(127 \cdot 1 \\
\text { to } 295 \cdot 2)\end{array}$ & $\begin{array}{r}48 \cdot 4^{*}(46 \cdot 1 \text { to } \\
50 \cdot 7)\end{array}$ & $\begin{array}{r}-5.7^{*}(-7.2 \text { to } \\
-4.0)\end{array}$ \\
\hline Animal contact &.. &.. & .. & $\begin{array}{r}403.5(299.5 \\
\text { to } 527.6)\end{array}$ & $\begin{aligned}-6 \cdot 3^{*}(-10 \cdot 6 \text { to } \\
-1 \cdot 4)\end{aligned}$ & $\begin{array}{r}-36.4^{*}(-39 \cdot 0 \text { to } \\
-33.5)\end{array}$ \\
\hline Venomous & $\begin{array}{r}2274 \cdot 1(2204 \cdot 9 \\
\text { to } 2346 \cdot 6)\end{array}$ & $2 \cdot 6(-2 \cdot 1$ to $6 \cdot 8)$ & 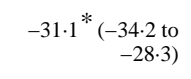 & $\begin{array}{r}153 \cdot 1(110 \cdot 5 \\
\text { to } 201 \cdot 1)\end{array}$ & $\begin{array}{r}-7.5 *(-12 \cdot 6 \text { to } \\
-2 \cdot 2)\end{array}$ & $-37 \cdot 1 * \begin{array}{r}(-40.5 \text { to } \\
-33 \cdot 6)\end{array}$ \\
\hline Non-venomous & $\begin{array}{r}12089 \cdot 8(10 \\
522 \cdot 9 \text { to } 13 \\
775 \cdot 5)\end{array}$ & $\begin{array}{r}-5 \cdot 6(-10 \cdot 9 \text { to } \\
0 \cdot 0)\end{array}$ & $\begin{array}{r}-32.8 *(-35.9 \text { to } \\
-29.6)\end{array}$ & $\begin{array}{r}250.5(183.9 \\
\text { to } 337.8)\end{array}$ & $\begin{array}{r}-5 \cdot 4(-10 \cdot 9 \text { to } \\
0 \cdot 6)\end{array}$ & $\begin{array}{r}-36.0^{*}(-38.9 \text { to } \\
-32.8)\end{array}$ \\
\hline Foreign body & .. & .. & .. & $\begin{array}{r}254 \cdot 8(186 \cdot 8 \\
\quad \text { to } 325 \cdot 0)\end{array}$ & $\begin{array}{r}22 \cdot 1 *(16 \cdot 2 \text { to } \\
29 \cdot 0)\end{array}$ & $\begin{array}{r}-19 \cdot 4^{*}(-23 \cdot 3 \text { to } \\
-15 \cdot 0)\end{array}$ \\
\hline $\begin{array}{l}\text { Pulmonary aspiration and foreign } \\
\text { body in airway }\end{array}$ & $\begin{array}{r}887.8(873.4 \text { to } \\
902.7)\end{array}$ & $\begin{array}{r}42.5^{*}(39.7 \text { to } \\
45.5)\end{array}$ & $\begin{array}{r}-4 \cdot 6 *(-6 \cdot 3 \text { to } \\
-2 \cdot 8)\end{array}$ & $\begin{array}{r}50 \cdot 2(38 \cdot 4 \text { to } \\
64 \cdot 6)\end{array}$ & $\begin{array}{r}-2 \cdot 7(-10 \cdot 5 \text { to } \\
7 \cdot 4)\end{array}$ & $\begin{array}{r}-33.7^{*}(-38.6 \text { to } \\
-27.2)\end{array}$ \\
\hline Foreign body in eyes & $\begin{array}{r}1164 \cdot 8(737 \cdot 5 \\
\text { to } 1586 \cdot 1)\end{array}$ & $\begin{array}{r}41.5 *(37.7 \text { to } \\
46.7)\end{array}$ & $0.4(-1.5$ to 2.3$)$ & $\begin{array}{r}60 \cdot 3(35.0 \text { to } \\
91 \cdot 7)\end{array}$ & $\begin{array}{r}30 \cdot 6 *(26 \cdot 0 \text { to } \\
34 \cdot 9)\end{array}$ & 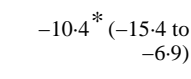 \\
\hline Foreign body in other body part & $\begin{array}{r}3912 \cdot 3(3465 \cdot 6 \\
\text { to } 4383 \cdot 6)\end{array}$ & $\begin{array}{r}42 \cdot 0 *(34 \cdot 0 \text { to } \\
49 \cdot 6)\end{array}$ & $\begin{array}{r}-4 \cdot 0 *(-8 \cdot 3 \text { to } \\
-0 \cdot 2)\end{array}$ & $\begin{array}{r}144 \cdot 3(108 \cdot 0 \\
\quad \text { to } 186 \cdot 5)\end{array}$ & $\begin{array}{r}30 \cdot 1 *(23 \cdot 9 \text { to } \\
36 \cdot 9)\end{array}$ & 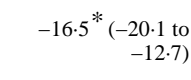 \\
\hline Other unintentional injuries & $\begin{array}{r}51132 \cdot 0(49 \\
359 \cdot 0 \text { to } 52 \\
946 \cdot 0)\end{array}$ & $\begin{array}{r}45 \cdot 2^{*}(43.1 \text { to } \\
47 \cdot 7)\end{array}$ & $\begin{array}{r}-3.9 *(-5 \cdot 2 \text { to } \\
-2.5)\end{array}$ & $\begin{array}{r}2589 \cdot 5 \\
(1905 \cdot 1 \text { to } \\
3445 \cdot 3)\end{array}$ & $\begin{array}{r}34.2 *(29.7 \text { to } \\
39 \cdot 1)\end{array}$ & $\begin{array}{r}-16 \cdot 0^{*}(-18 \cdot 5 \text { to } \\
-13 \cdot 3)\end{array}$ \\
\hline $\begin{array}{l}\text { Self-harm and interpersonal } \\
\text { violence }\end{array}$ &.. & .. & .. & $\begin{array}{r}1053 \cdot 5 \\
(778 \cdot 0 \text { to } \\
1388 \cdot 0)\end{array}$ & $1 \cdot 8(-5 \cdot 0$ to $10 \cdot 5)$ & $\begin{array}{r}-34 \cdot 4^{*}(-38 \cdot 5 \text { to } \\
-29 \cdot 2)\end{array}$ \\
\hline Self-harm & $\begin{array}{r}5940 \cdot 8(5845 \cdot 2 \\
\text { to } 6051 \cdot 2)\end{array}$ & $\begin{array}{r}21.5 *(18 \cdot 5 \text { to } \\
24 \cdot 2)\end{array}$ & 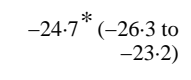 & $\begin{array}{r}231 \cdot 6(167 \cdot 0 \\
\text { to } 307 \cdot 3)\end{array}$ & $-1.7(-6.9$ to 5.4$)$ & $\begin{array}{r}-39 \cdot 0^{*}(-42 \cdot 2 \text { to } \\
-34 \cdot 8)\end{array}$ \\
\hline Interpersonal violence &.. & .. &.. & $\begin{array}{r}821.9(610 \cdot 4 \\
\quad \text { to } 1077.7)\end{array}$ & $2 \cdot 8(-4 \cdot 5$ to $12 \cdot 0)$ & $\begin{array}{r}-33.0^{*}(-37.4 \text { to } \\
-27.3)\end{array}$ \\
\hline Assault with firearm & $\begin{array}{r}3870 \cdot 2(3765 \cdot 2 \\
\text { to } 3974 \cdot 2)\end{array}$ & $\begin{array}{r}46 \cdot 7^{*}(42 \cdot 6 \text { to } \\
50.5)\end{array}$ & $\begin{array}{r}-3.2 *(-5.9 \text { to } \\
-0.7)\end{array}$ & $\begin{array}{r}163 \cdot 1(120 \cdot 0 \\
\text { to } 216 \cdot 6)\end{array}$ & $\begin{array}{r}21.9 *(14.8 \text { to } \\
29.8)\end{array}$ & $\begin{array}{r}-21 \cdot 1 *(-25 \cdot 4 \text { to } \\
-16 \cdot 5)\end{array}$ \\
\hline Assault with sharp object & $\begin{array}{r}6078 \cdot 6(5868 \cdot 9 \\
\text { to } 6311 \cdot 1)\end{array}$ & $\begin{array}{r}41.9 *(36 \cdot 4 \text { to } \\
47 \cdot 2)\end{array}$ & $\begin{array}{r}-7.0 *(-10.3 \text { to } \\
-3.7)\end{array}$ & $\begin{array}{r}172 \cdot 3(124 \cdot 9 \\
\text { to } 233 \cdot 2)\end{array}$ & $\begin{array}{r}18.5 *(10 \cdot 8 \text { to } \\
28.0)\end{array}$ & $\begin{array}{r}-22.9^{*}(-27.6 \text { to } \\
-17.2)\end{array}$ \\
\hline Assault by other means & $\begin{array}{r}10133 \cdot 4 \\
(9835 \cdot 8 \text { to } 10 \\
448 \cdot 3)\end{array}$ & $\begin{array}{r}21 \cdot 4 *(18 \cdot 8 \text { to } \\
23 \cdot 7)\end{array}$ & $\begin{array}{r}-17.9 *(-19 \cdot 3 \text { to } \\
-16 \cdot 6)\end{array}$ & $\begin{array}{r}486 \cdot 5(364 \cdot 2 \\
\text { to } 635 \cdot 7)\end{array}$ & $\begin{array}{r}-6 \cdot 4(-12 \cdot 9 \text { to } \\
2 \cdot 4)\end{array}$ & $\begin{array}{r}-38 \cdot 8 *(-42 \cdot 9 \text { to } \\
-33 \cdot 4) \\
\end{array}$ \\
\hline $\begin{array}{l}\text { Forces of nature, war, and legal } \\
\text { intervention }\end{array}$ & .. &.. & .. & $\begin{array}{r}3933 \cdot 7 \\
(1860 \cdot 7 \text { to } \\
7821 \cdot 0)\end{array}$ & $\begin{array}{r}-50 \cdot 7^{*}(-57 \cdot 7 \text { to } \\
-40 \cdot 2)\end{array}$ & $\begin{array}{r}-67 \cdot 9^{*}(-72 \cdot 6 \text { to } \\
-61 \cdot 0)\end{array}$ \\
\hline Exposure to forces of nature & $\begin{array}{r}14335 \cdot 5 \\
(7133 \cdot 3 \text { to } 28 \\
680 \cdot 1)\end{array}$ & $\begin{array}{r}8 \cdot 2(-4 \cdot 6 \text { to } \\
29 \cdot 0)\end{array}$ & $\begin{array}{r}-37 \cdot 4 *(-45 \cdot 6 \text { to } \\
-24 \cdot 5)\end{array}$ & $\begin{array}{r}556 \cdot 8(280 \cdot 6 \\
\text { to } 1117 \cdot 0)\end{array}$ & $\begin{array}{r}-0.7(-15 \cdot 1 \text { to } \\
22 \cdot 3)\end{array}$ & $\begin{array}{r}-36 \cdot 7^{*}(-45 \cdot 8 \text { to } \\
-22 \cdot 0)\end{array}$ \\
\hline
\end{tabular}




\begin{tabular}{|c|c|c|c|c|c|c|}
\hline & $\begin{array}{l}\text { Prevalent cases } \\
\text { in 2013 }(x \\
1000)\end{array}$ & $\begin{array}{l}\text { Percentage } \\
\text { change in } \\
\text { prevalence } \\
\text { from } 1990 \text { to } \\
2013\end{array}$ & $\begin{array}{l}\text { Percentage } \\
\text { change in age- } \\
\text { standardised } \\
\text { prevalence from } \\
1990 \text { to } 2013\end{array}$ & $\begin{array}{l}\text { YLDs in } \\
2013(x \\
1000)\end{array}$ & $\begin{array}{l}\text { Percentage } \\
\text { change in YLDs } \\
\text { from } 1990 \text { to } \\
2013\end{array}$ & $\begin{array}{l}\text { Percentage change } \\
\text { in age- } \\
\text { standardised } \\
\text { YLDs from } 1990 \\
\text { to } 2013\end{array}$ \\
\hline $\begin{array}{l}\text { Collective violence and legal } \\
\text { intervention }\end{array}$ & $\begin{array}{r}43478 \cdot 1(23 \\
580 \cdot 2 \text { to } 77 \\
304 \cdot 6)\end{array}$ & $\begin{array}{r}-27.0 *(-32 \cdot 2 \\
\text { to }-17 \cdot 3)\end{array}$ & $\begin{array}{r}-46 \cdot 7^{*}(-51 \cdot 2 \text { to } \\
-40 \cdot 8)\end{array}$ & $\begin{array}{r}3376 \cdot 9 \\
(1547 \cdot 3 \text { to } \\
6784.6)\end{array}$ & 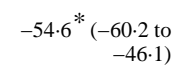 & $\begin{array}{r}-70.3^{*}(-74.2 \text { to } \\
-64.5)\end{array}$ \\
\hline
\end{tabular}

All data are reported with 95\% uncertainty intervals. YLDs=years lived with disability.

* Significant percentage change. 